\title{
Introduction
}

Nanotechnology has emerged as a pervasive technology with applications ranging from industry to healthcare. The medical application of nanotechnologies nowadays includes innovative approaches for therapies and diagnostics (theranostics), as well as nano-enabled biomaterials for tissue engineering and regenerative medicine. To perceive the pervasiveness of nanotechnologies in healthcare, a simple search in the Web of Science database as late as January 2020 for the keyword “nano" coupled to "therapy", "diagnostics", "medicine”, or "tissue engineering” resulted in over 200k entries, with a boost started in the early 2000s and still gaining increasing interest.

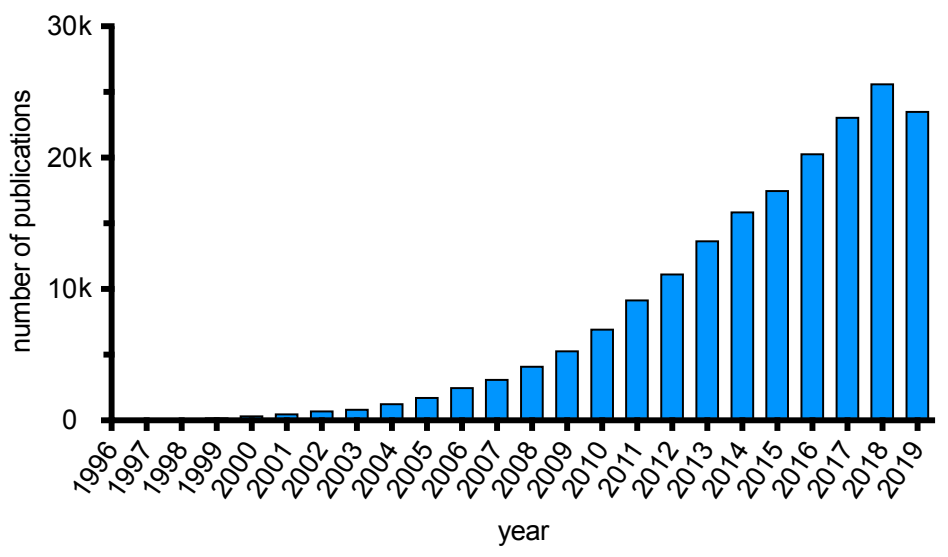

Figure 1. Trend of publications in the field of medical nanotechnologies. Data source: WoS, January 2020.

From a materials science perspective, the local manipulation of matter at the atomic and molecular scale results in materials exhibiting novel and significantly improved physical, chemical, and biological properties. The quest for new drug delivery systems, cell-compatible scaffolds, contrast agents, and medical tools for the treatment of tumors or neurodegenerative diseases, have pushed researchers to the fabrication of novel classes of nanomaterials. Aim of this book is to provide a comprehensive overview on the broad field of medical nanotechnologies. The reader will be primed on the physico-chemical fundamentals of bionanotechnologies, and will be walked through the most salient applications of nanomaterials in the fields of theranostics and tissue engineering. Importantly, the book will also pose emphasis on the open challenges and safety issues related to the implementation of nanotechnologies.

The book has been divided into four Sections. 
The first Section deals with nanosystems for controlled drug delivery. Dr. Sponchioni (Chapter 1) describes polymeric nanoparticles as drug delivery systems (DDS), while the group of Dr. Bellucci (Chapter 2) presents nanocarbon vectors for drug delivery. Dr. Mauri (Chapter 3) provides further insights on chemical functionalization strategies to improve the delivery performances of nanostructured systems. Optimization of nanosystems also demands for advanced analytical methods: Castiglione et al. (Chapter 4) disclose HR-MAS as a tool for optimizing drug release profiles. Optimization also passes through advanced in silico models, that are reviewed by Dr. Casalini (Chapter 5).

Section 2 presents the application of nanomaterials for advanced analytical techniques. Dr. Bonifacio (Chapter 6) presents a thorough insight on nanostructured substrates for SERS spectroscopy.

Section 3 deals with nanobiomaterials for tissue engineering and regenerative medicine. Romano et al. (Chapter 7) presents extracellular vesicles as tools for regenerative medicine. The group of Dr. Guarino (Chapter 8) gives an overview on a wide range of electro- and non-electro assisted spinning technologies for in vitro and in vivo applications. Nanoceramics also represent an important class of biomaterials for tissue engineering; Kohli and García-Gareta (Chapter 9) summarize the state of the art in the field.

Last, Section 4 deals with nanosafety and regulatory issues for nanomaterials in medicine. The group of Prof. Perale (Chapter 10) introduces the concepts of safety-by-design, as well as human and environmental risks associated to nanobiomaterials.

De Angelis et al. (Chapter 11), instead, focus on regulatory issues related to nanomaterials with specific focus on nanomedicine products.

We hope you will enjoy reading this book.

The Editors 


\title{
Chapter 1
}

\section{Polymeric Nanoparticles for Controlled Drug Delivery}

\section{Mattia Sponchioni ${ }^{1,2}$}

${ }^{1}$ Department of Chemistry and Applied Biosciences, Institute for Chemical and Bioengineering, ETH Zurich, Vladimir-Prelog-Weg 1, 8093 Zurich, Switzerland

${ }^{2}$ Department of Chemistry, Materials and Chemical Engineering "Giulio Natta", Politecnico di Milano, via Mancinelli 7, 20131 Milano, Italy

\begin{abstract}
While liposomes are the main representatives of the first generation of nanotherapeutics, that is to say nanosized vectors for the encapsulation and controlled release of therapeutics, polymer NPs are rapidly coming to the fore. This is mainly due to the possibility of finely controlling their physical and chemical properties, taking advantage of the advent of controlled polymerization techniques. Another important factor that justifies this growing interest is the development of stimuliresponsive polymers, which can be exploited for a precise drug targeting by exploiting environmental signals. Therefore, the necessity of smart drug delivery systems for a second generation of nanotherapeutics, and in particular for the delivery of nucleic acids, proteins or other biotherapeutics could be covered by these stimuli-responsive NPs in the near future. However, the long road towards the final clinical application should be carefully considered during the design of a novel nanoformulation. Here, the frontiers in the synthesis of smart polymer NPs to direct a drug to the desired site of action are presented in the context of their implication in biological systems. Finally, the stages for a nanotherapeutic before gaining the approval for clinical applications are discussed in order to understand the current regulatory framework for these systems.
\end{abstract}

Keywords: Polymer; Nanoparticles; Drug Delivery; Targeting; Stimuli-Responsive; Regulation; Clinical Trials 


\section{General Concepts and Synthetic Strategies}

The idea of controlled drug delivery arises from the dream of selectively addressing a bioactive compound to a specific target area in the body, in order to maximize its therapeutic effect while minimizing side effects. In recent years, nanometer-sized drug delivery systems, and specifically nanoparticles (NPs) able to load and mediate the release of therapeutically active compounds, have experienced growing attention due to the several advantages they offer compared to traditional ways of drug administration[1,2]. In particular, NPs can ideally take advantage of all of the possible administration routes, including oral, mucosal, transdermal, subcutaneous and intravenous. The limitation is represented by the biological barriers that the NPs have to cross before reaching the target, which determines the efficacy of the formulation[3, 4]. Following this consideration, the optimal administration route would be the oral one, being the less invasive. However, this is often characterized by a poor adsorption by the gastrointestinal mucosa and by a harsh environment for the NPs, especially in terms of $\mathrm{pH}$. This is why parenteral administrations, including subcutaneous, intravenous and transdermal, are so far the most explored and characterized by the highest drug bioavailability[5]. Following this administration route, drug delivery through NPs ensures the maintenance of the drug concentration in a desired therapeutic window over a prolonged period of time, thus ideally reducing the amount of drug and dosages required[6]. Additionally, it reduces the side effects associated to the traditional formulations based on organic solvents and surfactants, with an overall increase in the patient compliance[7].

Different kinds of NPs have been designed during the years as drug delivery systems. In general, a distinction is worthy between inorganic and organic NPs. Among the former, iron oxide [8, 9] and silica[10, 11] NPs represent the golden standard. However, organic NPs and in particular polymeric NPs play a key role. The latter offer several advantages including the possibility of tuning their physico-chemical properties as well as of introducing specific functionalization, which makes them suitable for loading and controlling the release of different active principles. 
The NP efficacy in the controlled drug delivery is indeed strongly affected by their properties, and in particular by the size. Nanovectors aimed at systemic administration, for example, should be in the range 30-300 $\mathrm{nm}$ in size. Indeed, NPs smaller than $10 \mathrm{~nm}$ are below the renal threshold for direct excretion, and hence would be eliminated soon after the injection. This brings about a reduced circulation time and hence a limited possibility of reaching the target site of action[12-14]. On the other hand, NPs bigger than $300 \mathrm{~nm}$ introduce the severe risk of thrombosis, since the smallest capillaries in the body have a diameter in the order of few hundreds of nanometers. Additionally, such nanovectors are more likely amenable for opsonization operated by the macrophages of the reticuloendothelial system (RES) or by hepatic Kupffer cells, leading again to a reduced circulation time $[15,16]$. The NP circulation time in the bloodstream is also strongly affected by their surface composition. It is known that nanovectors in a biological environment undergo the so-called protein corona effect soon after their infusion, being covered by a layer of adsorbed proteins that facilitates their recognition by the macrophages[17, 18]. Nowadays, the most adopted strategy to avoid this recognition is the surface modification with polyethylene glycol (PEG), an uncharged and hydrophilic polymer. In fact, PEG creates a hydration layer over the NP surface that hides them from the macrophages recognition. This strategy, commonly referred to as PEGylation, is therefore largely employed not only in the realization of "stealth" nanovectors, but also to increase the water stability of lipophilic compounds, proteins and antibodies, thus improving their efficacy[19, 20]. However, several drawbacks have only recently been discovered when using PEG in the stabilization of polymer NPs. In particular, the immune system increases the production of antibodies able to specifically bind to PEG after repeated treatments with PEGylated compounds. This process gives rise to the so-called accelerated blood clearance $(\mathrm{ABC})$ of such functionalized therapeutics[19, 21-23]. Additionally, recent clinical trials highlighted allergic reactions and/or hypersensitivity to PEG for a significant number of patients[24-26], thus pushing the literature to an extensive research effort to find valuable substitutes to PEG[27-30]. 
So far, zwitterionic polymers represent the most promising alternative to PEG in the stabilization of polymer NPs in water[31-33]. Indeed, the strong electrostatic interactions among the charged groups in these polymers determine the formation of a hydration layer, over the NP surface, able to prevent the nonspecific adsorption of biological macromolecules[34-36]. This determines not only a high stability in biological environment but also the non-specific elimination of the vector.

It is evident from these considerations, that the proper design of the polymer NPs represents a crucial point in determining the circulation time, target selectivity and drug delivery efficacy[37]. The synthesis of these nanocolloids is usually obtained through either physical methods from preformed polymers or chemical methods. Among the physical methods, it is worth citing the emulsionevaporation process and the nanoprecipitation. The former relies on the polymer dissolution in a water-immiscible organic solvent (e.g. chloroform, ethyl acetate, toluene), followed by the emulsification of this organic phase in water with surfactants. Finally, the NP latex is obtained following the evaporation of the organic solvent. On the other hand, in the nanoprecipitation method, the polymer is dissolved in a water-miscible organic solvent (e.g. ethanol, dimethylformamide, dimethylsulfoxide). The organic phase is added to a water suspension of surfactant micelles under turbulent mixing conditions. Finally, the organic solvent is removed through dialysis. It is evident that both processes rely on the use of organic solvents as well as of surfactants, which may be harmful when injected into the body. In addition, the physical processes suffer the limitation of a poor solid content in the final NP suspension and the use of complex mixing devices to achieve proper turbulent conditions. Despite these drawbacks, the physical methods are necessary in few occasions. It is the case for example of the synthesis of biodegradable NPs. Biodegradable NPs, mainly obtained from aliphatic polyesters, are of paramount importance for drug delivery. In fact, the polyester chains they are made up of can undergo hydrolytic degradation in aqueous environments, thus ensuring the avoidance of any polymer accumulation into the body[38]. 
Industrially, high molecular weight polyesters are produced via the ring opening polymerization (ROP) of cyclic monomers (e.g. lactide, glycolide, $\varepsilon$-caprolactone) and are obtained as bulk materials. Therefore, a common strategy to formulate the bulky material in NPs is based on either the emulsionevaporation or the nanoprecipitation process.

To obtain polymer NPs with a size range suitable for systemic administration, it is also possible to resort to the chemical methods, and in particular to emulsion polymerization. This is actually a wellestablished technique to obtain PEGylated NPs. In fact, PEG-methacrylate derivatives (i.e. PEGMA) can be used as reactive surfactants, also known as surfmers[39], in the emulsion polymerization of lipophilic monomers. In this way, the particle surface is covered with PEG tethers that are functional in increasing its circulation time into the bloodstream. Additionally, the surfmer is chemically bound to the NP core, and hence its desorption, which may cause latex aggregation, is prevented. A step forward to obtain biodegradable NPs via chemical methods is the combination of ROP and radical chemistry in the so-called "macromonomer method". In particular, short oligoester macromonomers can be obtained via ROP initiated by a vinyl group bearing alcohol (e.g. 2-hydroxyethyl methacrylate, HEMA), as shown in Figure 1a. The produced macromonomer can be further reacted via free radical emulsion polymerization to obtain NPs that are structurally composed of polymer chains with a peculiar comb-like structure, comprising a polyHEMA backbone and biodegradable oligoester lateral chains[40, 41]. This architecture enables the control over the degradation time of the formulation, which can be modulated by changing the number of repeating units in the lateral chains as well as the monomer adopted in the ROP[42]. From preliminary in vivo results, this kind of NPs demonstrated a promising tool to formulate the poorly soluble antitumor drug Paclitaxel (PTX)[43, 44]. This formulation reached the same therapeutic index as the commercialized PTX formulation obtained with the emulsifier Cremophor $\mathrm{EL}^{\circledR}$, but avoided the side effects related to the use of this latter excipient. 
A further degree of control in the NP design has been introduced with the advent of the controlled radical polymerization techniques, mainly nitroxide-mediated polymerization (NMP)[45, 46], atom transfer radical polymerization (ATRP) [47, 48] and reversible addition-fragmentation chain transfer (RAFT) polymerization[49-52]. These techniques enable a precise control over the polymer microstructure and complex polymer architectures can be accessed. These features can be exploited to synthesize modular block copolymers for the individual tuning of the different NP properties (e.g. size, degradation time, molecular weight) and hence for the specific optimization of the nanovectors for drug delivery. In particular, RAFT polymerization or ATRP can be conveniently combined with ROP to obtain amphiphilic block copolymers self-assembled into polymer NPs able to degrade under physiological conditions. This combination can be achieved through a variety of strategies, as extensively reported in [53]. However, the macromonomer method is still the way providing the highest number of degrees of freedom in the NP design. In fact, the RAFT polymerization enables the control over the number of the hydrophilic as well as of the hydrophobic repeating units in the corresponding block of the copolymer, while the ROP controls the length of the oligoester lateral chains. By acting on these parameters it is possible to tune the NP surface chemistry, size[54] and degradation time[32], respectively (see Figure 1b-c). In addition, the high level of control over the block copolymer structure facilitates its self-assembly in aqueous environment. This is of high interest since the NP formulation and drug loading can be obtained with rudimental apparatus (i.e. a syringe and a needle) and directly at the bed of the patient, thus avoiding premature NP degradation and drug release[55]. 
a) Macromonomer Synthesis

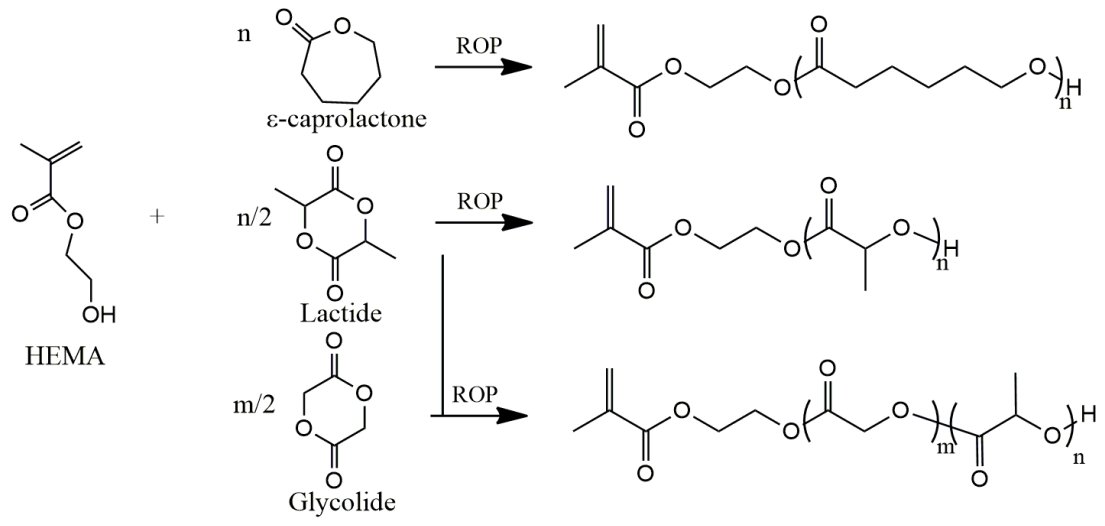

b) Controlled Radical Polymerization (CRP)

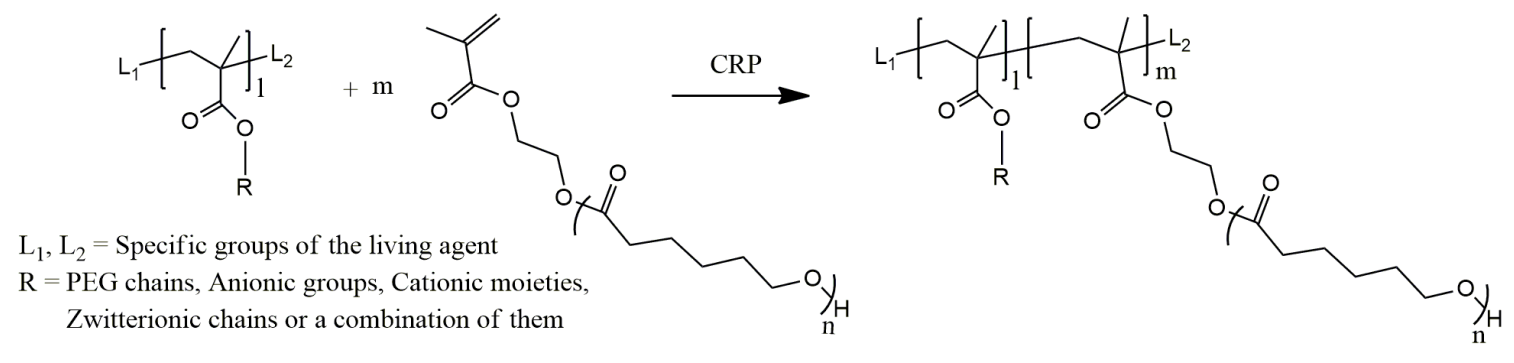

c) Self-assembly of the block copolymers

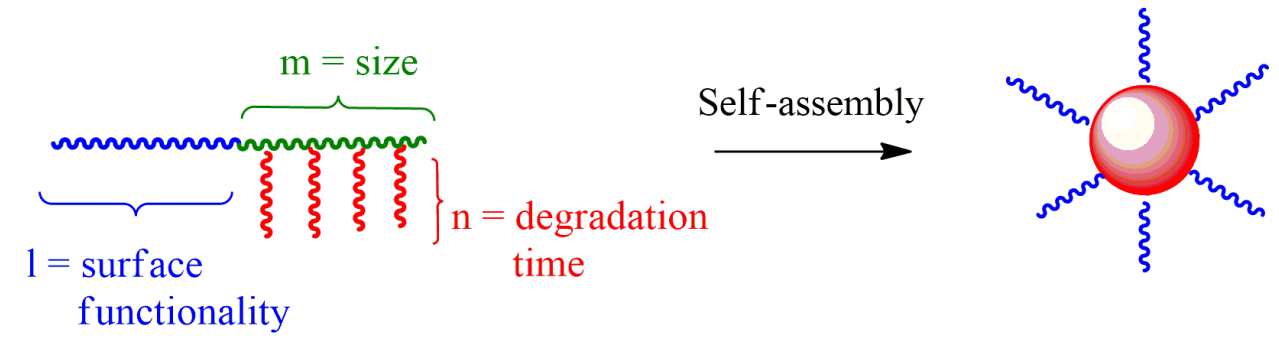

Figure 1. a) ROP exploited to produce short oligoester chains functionalized with the HEMA vinyl bond starting from \&-caprolactone, lactide or glycolide in the so-called "macromonomer method". b) Synthesis of amphiphilic biodegradable block-copolymers through the combination of controlled radical polymerization (i.e. ATRP, RAFT polymerization or NMP) and ROP. c) Comb-like copolymers from the combination of ROP and CRP for the precise control over the NP properties, including surface functionality, size and degradation time by modulating the hydrophilic repeating unit(s), number of hydrophobic units (m) and length of oligoester chain (n), respectively. 


\section{Polymer NPs for Controlled Drug Delivery}

The increasing attention to polymer NPs in the field of drug delivery is justified by the necessity of finding sustainable and harmless excipients for different therapeutics, possibly able to localize the drug release at the desired site and with the desired kinetic. Traditional excipients such as organic solvents and surfactants indeed suffer the limitation of induced cytotoxicity and incapacity to modulate the release of the therapeutic. This is particularly problematic when their nonspecific action is cause of serious side effects. On the other hand, a proper excipient is required to formulate a therapeutic, either to increase its bioavailability in an aqueous environment as in the case of a lipophilic drug, or to prevent it from premature inactivation/degradation, as in the case of genetic material. One of the main fields where polymer NPs are being tested as drug delivery vehicles is that of cancer treatment. Most anticancer drugs have indeed a very poor solubility in water, and their formulation in aqueous environments is problematic. In addition, chemotherapy suffers from poor specificity of the drugs used. Due to their potent action, this is often cause of side effects that have severe impact on the patient compliance. On the other hand, the antitumor drug encapsulation in a polymer vector prevents these side effects in healthy tissues. As a representative example, the marketed formulation of Trabectedin, Yondelis ${ }^{\circledR}$, is notoriously source of Grade 3 and 4 neutropenia, rhabdomyolysis and phlebitis at the site of injection when administered intravenously[56, 57]. This requires the administration through a central venous catheter in a large vein, even if residual phlebitis has been reported also in this case[58]. On the other hand, preliminary studies demonstrated that Trabectedin encapsulation in polymer NPs enables a prolonged release and hence a reduced number of required administrations. More importantly, the drug showed the same antitumor activity compared to Yondelis ${ }^{\circledR}$ but a better toxicological profile. In particular, the drug encapsulation in polymer NPs considerably mitigated both hyperplasia and epidermal lesion at the site of injection[30, 55]. 
Another application that polymer NPs are more and more considered for is the delivery of a novel class of biotherapeutics, including nucleic acids and proteins. The market for this therapeutics is rapidly growing in the last years and a huge research effort is being spent in the development of a suitable delivery system. The scope for this excipient is the avoidance of premature degradation for the active principle, mainly operated by serum endonucleases. In addition, the vector should guarantee an efficient cell internalization, since these biotherapeutics operate mainly in the cytosol or in the cell nucleus. In the case of the nucleic acids, the delivery is mainly obtained with modified viruses, such as adenoviruses, retroviruses and lentiviruses. However, these viral vectors have not received the approval from the Food and Drug Administration because of their carcinogenicity and immunogenicity exhibited in the clinical trials, combined with difficult production procedures that lead to a broad batch-to-batch variability $[59,60]$. These problems can be solved with the use of synthetic non-viral vectors. Among this category, the most studied delivery systems are cationic NPs able to form the so-called polyplexes after complexation with the anionic genetic material. In particular, NPs comprising tertiary amines able to protonate under physiological conditions are largely employed in the literature, as extensively reviewed in [61].

Despite the huge benefits in loading different therapeutics in polymeric NPs, one of the main issues is how to address them to the target site, i.e. where the drug is required. To achieve this goal, two targeting strategies can be exploited. The former, applicable to solid tumors, relies on their specific pathophysiological conformation. In particular, solid tumors are commonly associated with enhanced vascular permeability and poor lymphatic drainage, which enhance the extravasation of macromolecules larger than $40 \mathrm{kDa}$ and their consequent accumulation, respectively[62, 63]. This process is called enhanced permeability and retention (EPR) effect and is schematically depicted in Figure 2a. The EPR effect is a typical passive targeting approach, since it exploits a passive migration of the macromolecules and/or NPs in the tumor interstitial space rather than their active interaction with cancer cells. 
Despite the great popularity of the EPR effect as a targeting mechanism, some recent experiments are raising questions on its effectiveness. First of all, the high permeability of tumor vessels makes that the interstitial fluid pressure (IFP) is almost the same as that in the blood vessels. This suppresses any pressure gradients helpful in pushing the NPs to extravasate from the vessels to the interstitial space[37]. The NP migration is therefore governed only by diffusion, which is notoriously slow for large NPs, while the convective flow is almost completely suppressed[64-66]. This makes that the percentage of inoculated NPs that effectively accumulate in a solid tumor via EPR effect is indeed very low. In addition, the EPR effect was demonstrated successful in small rodent models, while fails in clinical trials. To cite a famous example, Abraxane ${ }^{\circledR}$, an albumin-bound PTX formulation, showed an extremely promising pharmacokinetic behavior in preclinical studies. The formulation indeed accumulated in murine models of solid tumors more selectively than the commercial Taxol. However, these benefits are not translated with the same effectiveness to the clinic[67, 68]. The reason behind this failure is the substantial difference between a murine and a human tumor. This difference is well depicted in a review by Danhier[69]. First, the former is larger and grows faster compared to a human tumor. In a mouse, a tumor can reach a mass up to the $10 \%$ of the total body weight. This of course determines a higher filtration operated by the tumor and then an enhanced accumulation of macromolecules, with a better pharmacokinetic behavior. In addition, a human tumor shows further key differences, as schematically depicted in Figure $\mathbf{2 b}$. These include the lack of fenestrations in the endothelium, a denser extracellular matrix and the presence of hypoxic regions. These remarkable differences require the set up of a more coherent model for the preclinical pharmacokinetic study and hence a proper reengineering of the polymer NPs to better exploit the EPR effect for an efficient accumulation in the tumor. 


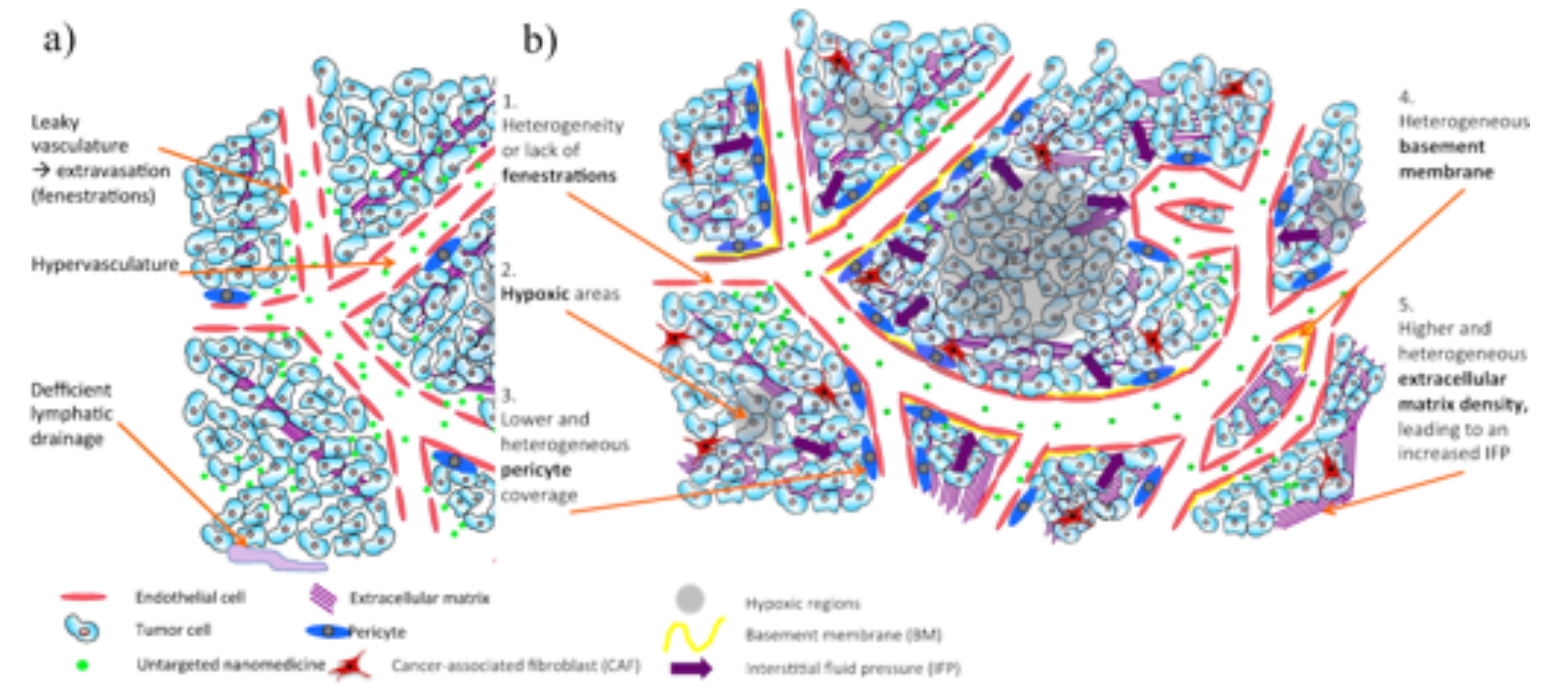

Figure 2. a) Schematic representation of the polymer NPs accumulation in solid tumors following the EPR effect. b) Peculiar properties of human tumors affecting the EPR effect. Reproduced with permission from [69]. Copyright Elsevier, 2016.

An alternative to passive targeting mechanisms is the functionalization of the NP surface with ligands able to selectively bind with receptors or antigens located on or in proximity of the target cells. This strategy is called active targeting. Different ligands can be exploited for targeting specific tissues. Among them, antibodies have been recognized effective in targeting tumors[70]. However, mainly due to their high molecular weight, nowadays they are more used to form armed antibodies, rather than for the functionalization of polymer NPs[71]. Small molecules can be adopted as ligands as well. For example, folate receptors are overexpressed on the surface of cancer cells. Therefore, folic acid is becoming a valuable tool to target tumors[72-74]. A significant example is provided by Poltavets et al. The authors synthesized docetaxel-loaded poly(lactic acid-co-glycolic acid) (PLGA) NPs and compared their internalization and antitumor activity in cervical carcinoma and breast adenocarcinoma cells in the case of folate-modified and unmodified surface. Folate-modified NPs showed apoptotic efficacy towards both cell lines comparable to that of free docetaxel and were more active than unmodified NPs. Also, the fraction of internalized NPs was higher for the modified $\mathrm{NPs}[75]$. 
Despite the number of successful active targeting examples is rapidly growing in the literature, they are all working in a pre-clinical stage, while a clinical proof of the effectiveness of such systems is still missing. Additionally, the functionalization of polymer NPs to realize an active targeting is still debated. In fact, while it is recognized that functionalized NPs are internalized into cells via endocytosis, a major hurdle is their escape from the endocytic pathway. This culminates with the degradative pathway in the lysosomes or with exocytosis. Therefore, the NP escape from the endosomes is crucial for an effective drug delivery[76]. Therefore, further studies are required to validate the active targeting strategy in increasing the therapeutic index of drug-loaded polymer NPs.

\subsection{Stimuli-Responsive Polymer NPs}

A relatively recent approach to control the drug release both in time and in space relies in the exploitation of physical stimuli to enhance the release rate. These stimuli comprise either temperature, $\mathrm{pH}$, ionic strength or magnetic field gradients and serve as switches to induce morphological changes in the polymer NPs or/and the rupture of specific chemical bonds. These so-called stimuli-responsive polymers were born from the necessity of mimicking the behavior of important biomacromolecules, which regulate important functions of living organisms in response to external inputs[77]. However, they soon found applications in the context of drug delivery.

Historically, the first stimuli-responsive NPs employed for the controlled delivery of therapeutics are those able to respond to $\mathrm{pH}$ changes in the surrounding. Upon variation in the $\mathrm{pH}$, the so-called $\mathrm{pH}-$ responsive NPs undergo morphological or conformational changes, mainly due to the ionization/deionization of weakly acidic or basic groups incorporated in the polymer matrix. This structure modification can be exploited to enhance the drug release rate from the NP in a specific site. This strategy takes advantage of the marked $\mathrm{pH}$ profile in living beings. The most remarkable example is the $\mathrm{pH}$ gradient in the gastrointestinal tract. In particular, the $\mathrm{pH}$ is extremely acidic (i.e. $\sim 1.0-2.5)$ in the stomach, while becoming basic (i.e. $6.4-7.8)$ in the intestine[78, 79]. 
This gradient is exploited for example by the polymeric excipient Eudragit ${ }^{\circledR}$, the trade name of a library of copolymers of esters of acrylic and methacrylic acid (i.e. $\mathrm{pKa}=4.5[80]$ ). Due to the presence of ionizable, methacrylic acid-derived units, these copolymers show a pH-dependent solubility in water and are employed to protect drugs administered via the oral route. In particular, in the stomach conditions, the copolymers are hydrophobic and form a protective coating, preventing the drug from premature degradation operated by gastric fluids. On the other hand, when reaching the basic $\mathrm{pH}$ in the intestine, the ionization of the methacrylic acid units determines the swelling of the copolymers and hence the release of the delivered drug[81-83]. When dealing with the systemic administration of drugs, $\mathrm{pH}$-responsive nanovectors may take advantage of the different $\mathrm{pH}$ observed in inflammatory sites, infections or tumors compared to that in normal tissues. Primary and metastasized tumors for example are often associated to a $\mathrm{pH}$ decrease from 7.4 (experienced in normal tissues) to 6 . This $\mathrm{pH}$ modification is due to the induced hypoxia, caused by the rapid expansion of the tumor mass and the consequent insufficient vascularization and oxygen provision. The metabolic environment in hypoxic regions induces the production of lactic acid and hence the acidification of the tumor interstitial space[84, 85]. By exploiting this phenomenon, it is then possible to produce polymer NPs comprising weak electrolytic groups with the appropriate $\mathrm{pKa}$, able to protect and retain an anticancer drug in healthy tissues and address its release only in the acidic tumor environment. One of the most studied strategy to achieve this behavior is the synthesis of polymer NPs comprising weak bases, and in particular tertiary amines. As an example, the Gao's group extensively studied PEG- $b$-poly(tertiary amine methacrylates) block copolymers to realize ultra-pHsensitive (UPS) NPs. These copolymers are self-assembled into NPs as long as the pH is above the tertiary amine $\mathrm{pKa}$, while disassemble into soluble unimers below this value. The precise control over the value of $\mathrm{pKa}$ and hence over the phase transition of the block copolymers can be obtained by varying the substituents to the amine groups and copolymerizing different tertiary amine methacrylates in the pH-responsive block. In this way, the authors were able to produce a library of NPs with tunable phase separation $\mathrm{pH}[86,87]$. 
The same group demonstrated that these $\mathrm{pH}$-responsive NPs could be an efficient tool for imaging as well. In particular, the authors loaded the NPs with a fluorescent dye. As long as the $\mathrm{pH}$ is above the $\mathrm{pKa}$ of the tertiary amines, the block copolymers are self-assembled in NPs and the fluorescence signal from the dye is quenched due to the Forster resonance energy transfer (HomoFRET) effect. However, upon access to the acidic tumor space $(\mathrm{pHe} \sim 6.5-6.8)$ or internalized in the endocytic organelles in the cancer endothelial cells $(\mathrm{pHi} \sim 5.0-6.0)$, the copolymer disassembly determines the activation of the fluorescence signal (Figure 3)[88]. In this way, a broad range of tumors can be selectively tracked, and this work pioneered the imaging-guided surgery conducted with suitable fluorescent cameras[89].
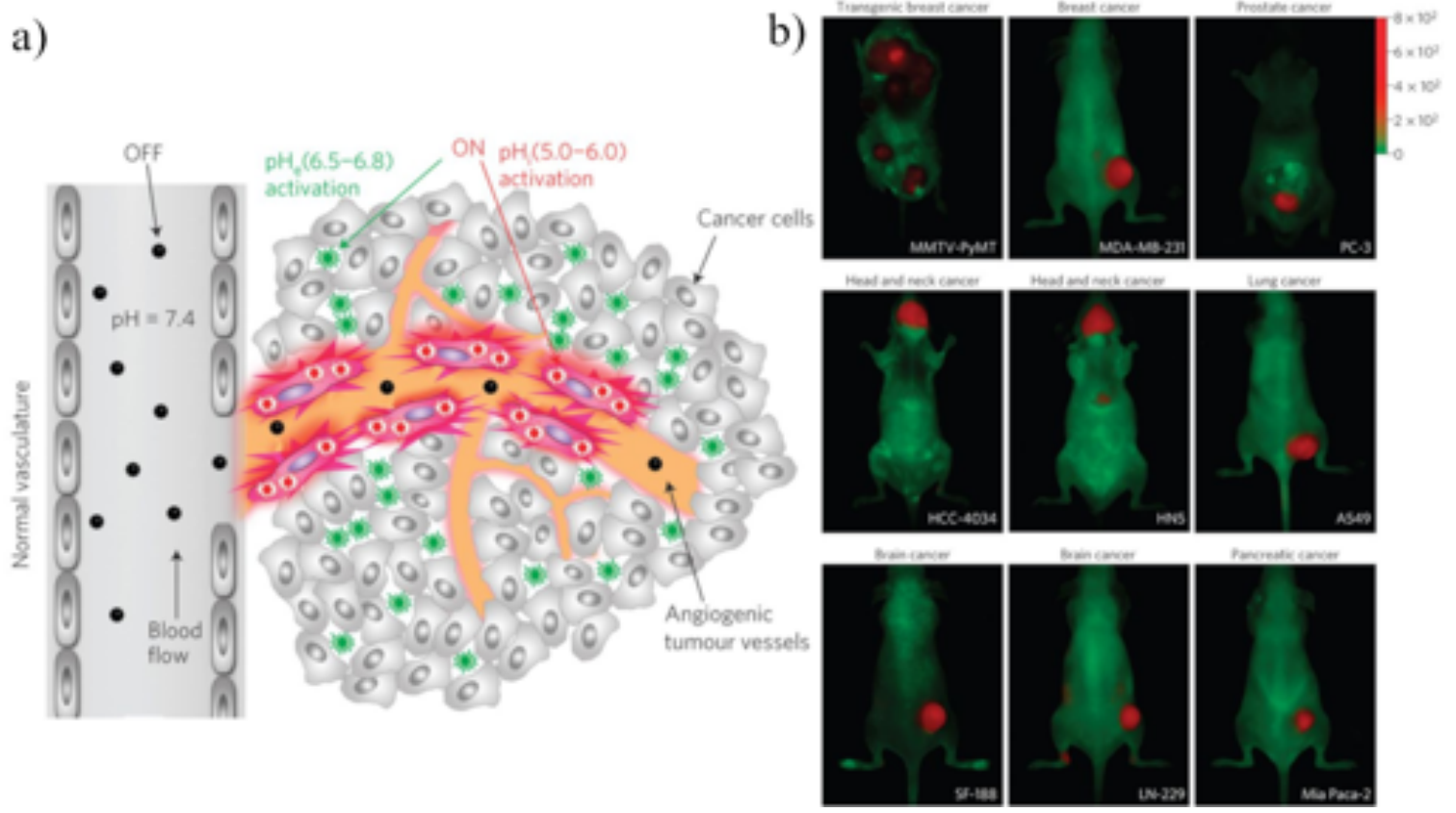

Figure 3. a) Mechanism of action of the $\mathrm{pH}$-sensitive nanoprobes. The probe stays $\mathrm{OFF}$ in the physiological $\mathrm{pH}$, while it is activated in the acidic conditions of the tumor interstitial space or in the endocytic organelles of the cancer endothelial cells. b) Selective tracking of different tumors using the pH-responsive NPs. The dye is activated selectively in the presence of the tumor. Reproduced with permission from [88]. Copyright Springer Nature, 2014. 
Another possibility to exploit variations in the $\mathrm{pH}$ to enhance the drug release is to chemically bind the drug molecule to the polymer matrix through a cleavable bond. Both acid-labile and base-labile linkages have been explored in the literature to bind a drug to the polymer NPs for $\mathrm{pH}$-responsive drug targeting. Acid-labile linkages are the most employed in cancer therapies, due to the acidic tumor environment. These include hydrazone, acetal, ketal and boronate ester bonds. Among them, the hydrazone linkage is the most appreciated, due to its stability in basic and physiological environments and facile synthesis. Lale et al. for example successfully increased the therapeutic activity towards breast cancer by linking the antitumor drug Doxorubicin to PEGylated NPs via the hydrazone bond[90]. The authors observed a $92 \%$ tumor regression compared to the $36 \%$ tumor regression in the case of free Doxorubicin, with minimal cardiotoxicity. This good result is also justified by a proper NP design to escape the endocytic pathway after cell internalization. The addition of tertiary amines in the polymer backbone enabled the induction of the so called "proton sponge effect" for the rupture of the lysosomes. In fact, the proton sequestration operated by the amines in the polymer keeps the proton pump active. This causes the retention of one anion (e.g. chlorine) per each sequestered proton. The increase in the electrolytic concentration increases the osmotic pressure that pushes water to flow from the cytosol into the lysosomes. This phenomenon leads to their acidification, swelling and ultimately to rupture[91].

Temperature is another stimulus commonly exploited for controlled drug release, also guided by the recent improvements in the instruments and techniques for precise temperature monitoring. Thermoresponsive NPs can exploit the naturally occurring temperature gradients to enhance the release of an entrapped drug. These are observed in inflammatory regions or in tumors, where the temperature can reach up to $42^{\circ} \mathrm{C}$, compared to $\sim 37^{\circ} \mathrm{C}$ in healthy tissues[92]. In addition, compared to $\mathrm{pH}$-responsive carriers, thermo-responsive drug delivery systems can be artificially activated by external heating or photoillumination. This provides an additional degree of freedom to induce the drug release in the desired site and at the desired time. 
Thermo-responsive polymers/solvent binary mixtures present a miscibility gap in the temperature vs. volume fraction phase diagram. In particular, two behaviors can be recognized. Polymers exhibiting an upper critical solution temperature (UCST) are soluble above their binodal curve while separate in a polymer-rich phase below it. The UCST is the maximum of this curve. On the other hand, polymers with a lower critical solution temperature (LCST) phase separate above their binodal curve, being the LCST the minimum of this curve[93, 94]. This latter class is the most exploited for realizing polymer NPs with thermal response in aqueous environments, mainly due to the higher convenience in tuning the LCST in a biologically relevant temperature range compared to UCST. The LCST thermal response arises from the breakage of water-polymer hydrogen bonding and the formation of more thermodynamically stable polymer-polymer interactions, with the release of water molecules in the bulk, upon heating above the cloud point (Tcp). The most studied thermo-responsive polymer for controlled drug release is poly(N-isopropylacrylamide) (PNIPAAm), mainly due to its LCST of 32 ${ }^{\circ} \mathrm{C}$, close to human body temperature, and poor sensitivity of its phase transition to external conditions such as polymer concentration, medium composition and $\mathrm{pH}[95]$. However, PNIPAAm brings about few drawbacks when used for biomedical applications. First, as for many acrylamides, the monomer shows significant cytotoxicity and hence the final product requires careful purification to avoid detrimental effects. In addition, the strong intrachain hydrogen bonds formed in the dehydrated state hinder the rehydration when the temperature is lowered, thus leading to a marked hysteresis that prevents a perfectly reversible "on-off" transition[96]. These drawbacks are currently reducing the interest towards PNIPAAm. Valuable alternatives are represented by poly(2-alkyl-2-oxazolines)[97, 98], poly(N-vinylcaprolactam)[99, 100] and poly(oligo ethylene glycol methacrylate)s (POEGMAs). These latter polymers are attracting considerable attention, due to the biocompatibility of the PEG substituents and the possibility of modulating the polymer LCST by changing the length of the PEG moieties[101, 102]. In addition, the Tcp can be finely modulated through the copolymerization with hydrophilic or hydrophobic monomers. 
In the former case, the copolymer is less prone to dehydration and the LCST is shifted towards higher values. On the other hand, when hydrophobic units are incorporated, the LCST is lowered. Lutz et al. demonstrated that the LCST of copolymers of two oligo(ethylene glycol)methyl ether methacrylates (OEGMAs) with different length of the PEG substitutent linearly varies with the copolymer composition[101]. Then, it is possible to access a whole range of Tcp by simply playing with the stoichiometry of the two monomers. This is of extreme importance for the realization of nanovectors suitable for very specific applications.

The most exploited strategy to realize LCST-based NPs for controlled drug release is the synthesis of block copolymers comprising a thermo-responsive segment and a hydrophobic block[93]. These systems form stable NPs in water, with the thermo-responsive segment forming the shell and the hydrophobic block the core of the colloids, as long as the temperature is below the Tcp. However, when the temperature is raised above the Tcp, the thermo-responsive shell phase separates collapsing over the core. This phenomenon leads to aggregation and to the formation of hydrophobic microstructures from which the release rate of an encapsulated therapeutic is enhanced. The reason for this accelerated drug release is still debated. The most accredited hypothesis is that a lipophilic drug can diffuse freely in the whole hydrophobic environment of the microstructures, thus avoiding intra-particle concentration gradients that could limit the solubilization equilibrium[103]. The work of Chung et al. pioneered this field. The authors developed PNIPAAm- $b$-poly(butyl methacrylate) based NPs and used them to encapsulate and control the release of Adriamycin. The tunable phase separation together with the reversibility of the NP aggregation allowed the authors to develop a system with a very high degree of control in the drug release rate. In particular, they demonstrated the possibility of achieving a pulsatile release by simply applying a step-wise temperature profile. In particular, Adriamycin was efficiently retained in the NP core below the Tcp and rapidly released once the temperature was raised above this threshold value[103]. Following this pioneer work, different examples of thermo-responsive drug delivery systems appeared in the literature, as reviewed in [93]. 
Of course, an important prerequisite remains the biodegradability of the NPs, in order to prevent their accumulation in the body. To achieve this goal, it is possible to exploit the macromonomer method. In particular, as shown by Sponchioni et al., a thermo-responsive macromolecular chain transfer agent (macro CTA) can be synthesized via RAFT polymerization. The phase separation can be finely tuned in this stage by copolymerizing OEGMAs with different molecular weight and defined mole ratio. On the other hand, the RAFT copolymerization ensures low interchain composition gradients[104], and hence a well-defined phase transition. The thermo-responsive macro CTA can be then chainextended with a biodegradable macromonomer obtained from the ROP of $\varepsilon$-caprolactone using HEMA as the initiator. The obtained amphiphilic block copolymers can be self-assembled in water to form NPs and loaded with PTX. The authors demonstrated the possibility of controlling the drug release rate following temperature stimulation as well as the degradability of the NP (see Figure 4)[105].

a)

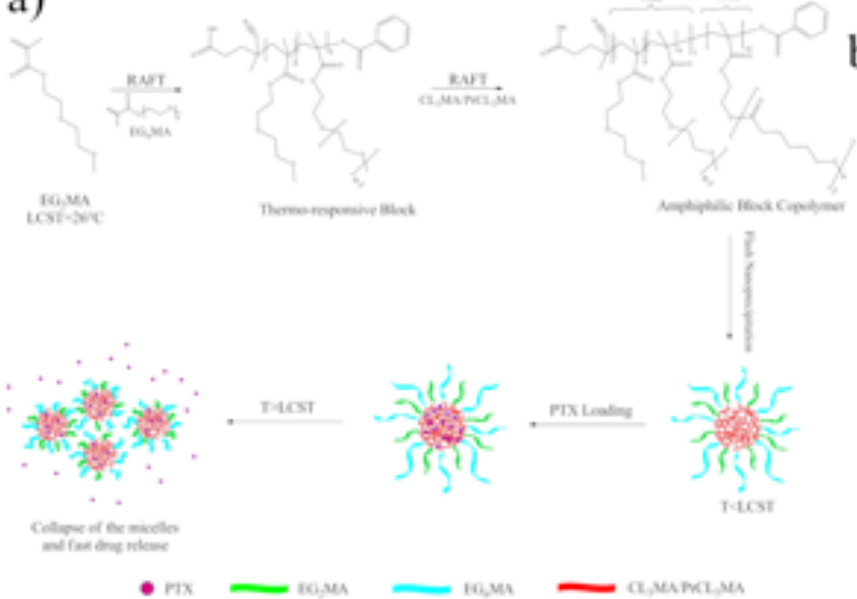

b)

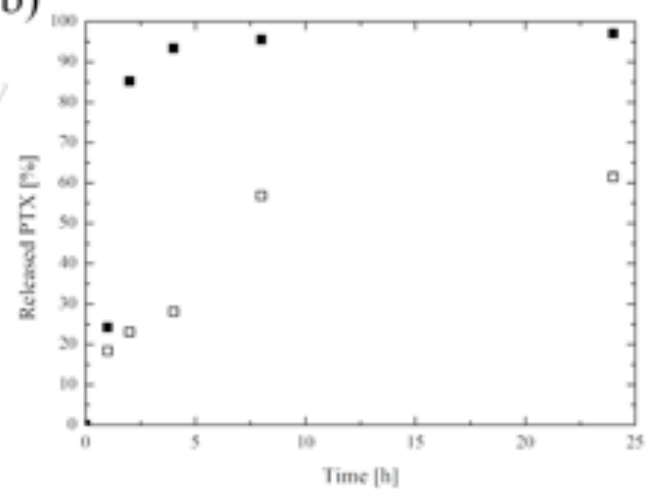

Figure 4. a) Schematic representation of the synthesis and phase behavior of thermo-responsive NPs obtained via a combination of ROP and RAFT polymerization. b) Control over PTX release measured at $6{ }^{\circ} \mathrm{C}(\square)$ and $40{ }^{\circ} \mathrm{C}(\square)$. Reproduced with permission from [105]. Copyright John Wiley and Sons, 2016. 
On the other hand, the synthesis of polymer NPs with a UCST behavior in physiological solutions is more challenging. The reasons are the shortage of polymers with this kind of phase behavior in aqueous environments as well as the sensitivity of the UCST to the polymer concentration and composition of the medium[106]. Still, the literature is growing in this field, with an approach that is dual compared to the LCST-type NPs. In fact, it is preferable in this case to prepare NPs from block copolymers comprising a hydrophilic block and a UCST segment. With this approach, stable NPs able to entrap and retain a hydrophobic drug are obtained below the copolymer Tcp. When the temperature is raised above this threshold, the inner core dissolves in water and the drug is instantaneously released. A nice example of this approach is provided by Li and coworkers, who synthesized PEG-b-poly(acrylamide-co-acrylonitrile) (P(AAm-co-AN)) block copolymers able to self-assemble into NPs with PEG on the surface and P(AAm-co-AN) segments in the inner core. The dissolution of this latter when the temperature is raised above its Tcp of $43{ }^{\circ} \mathrm{C}$ was exploited to instantaneously release the entrapped Doxorubicin. An enhanced antitumor activity for this thermoresponsive formulation was observed in a mouse model. In particular, the UCST NPs were injected intravenously and the Doxorubicin release induced in the tumor site by heating the region above the NP Tcp through microwave irradiation[107]. Another approach for UCST NPs is the use of the thermo-sensitive block of the copolymer to stabilize a hydrophobic NP core, similarly to the case of LCST NPs. This implies that the NPs are stored and administered at a temperature above the Tcp, where the stabilizing segments of the copolymers are water-soluble. The Tcp is then tuned to values slightly higher than the typical body temperature so that, once injected into the body, the formation of hydrophobic microaggregates leads to the sustained release of the entrapped drug.

The dual behavior between LCST and UCST NPs leads to opposite strategies for post processing and storage. For NPs stabilized by the thermo-responsive segment, the LCST NPs can be safely stored at room temperature and eventually freeze-dried. 
This is not possible for the UCST NPs, which on the other hand require the storage at temperature above their Tcp, with possible consequences in terms of reduced shelf life and premature release of the payload. On the other hand, when the thermo-responsive portions of the copolymers are used to fabricate the NP core, the LCST NPs disassemble at low temperature and should then be stored above their Tcp. Conversely, the UCST NPs can be safely stored at room temperature or even freezedried[93].

Finally, it is worth mentioning redox-responsive NPs. These are able to respond to changes in the redox potential of the environment. The most significant change in the redox potential in living organisms is experienced between the oxidizing extracellular environment and the reducing cytosol[108]. Thus, redox-sensitive NPs are mainly exploited for the intracellular delivery of therapeutics. The most typical approach to exploit this kind of stimulus relies on the incorporation of disulfide bonds in the NP core. These bonds prevent the NP disassembly in the oxidizing extracellular space. On the other hand, the high concentration of the reducing glutathione (GSH) in the intracellular environment causes the rapid breakage of the disulfide bond and in turn the disassembly of the NP core. This phenomenon is accompanied by the consequent, rapid release of the therapeutic entrapped in the NP core. Redox-sensitive NPs are currently attracting particular interest for the delivery of genetic materials (e.g. plasmid DNA, small interfering RNA, antisense oligonucleotides). These require a vector able to prevent their premature degradation caused by the plasma endonuclease as well as an efficient cell internalization. A representative example is provided by the work of Cavallaro et al., who developed a polyaspartamide non-viral gene delivery vector comprising ionizable amines for electrostatic interaction with DNA and disulfide bonds to hold the polymer chains assembled into NPs. These thiolpolyplexes prevented the metabolic degradation of the genetic material in the blood stream, while the reduction of the disulfide bridges operated by the GSH in the cytosol enabled the efficient release of DNA. On the other hand, the large number of amines provided a strategy for endosome escape exploiting the "proton sponge" effect[109]. 
Overall, stimuli-responsive NPs provide a valuable tool for the control of the drug release both in time and space. Of course, they rely on few hypothesis for a successful drug targeting. The first one is that the drug could be efficiently retained in the NP core in the absence of the stimulus. This prevents the drug dispersion during the systemic circulation and the related decrease in therapeutic efficacy and side effects. Then, the NP should present sufficient circulation time into the bloodstream to reach the target site. Finally, a proper strategy for a successful escape from the endocytic pathway should be included for the intracellular delivery of therapeutics. It should be clear from these points that the NP design plays a crucial role in leading to a proper therapeutic activity and drug targeting. In addition, the combination of different stimuli-responsive patches on the same NP would increase the number of degrees of freedom for an even more controlled drug release. Likely, the different research groups active in the drug delivery field will follow this direction in the near future.

\section{The Long Road from the Bench to the Clinic}

Nanosized drug delivery systems have rapidly grown in the last 30 years, with the first examples now available on the market. The first generation of nanotherapeutics, intended for the delivery of lipophilic drugs, is actually mainly represented by liposomes, by virtue of a research that dates back to the 1960s. However, the most successful formulation on the market is currently represented by Abraxane, a $10 \mathrm{~nm}$ albumin-bound PTX formulation. The high success of this nanoformulation is mainly due to the reduced side effects compared to Taxol ${ }^{\circledR}$. This enables a higher tolerability and hence higher dosages allowed, which translates into a higher therapeutic efficacy. This clinical success brought about the approval by the Food and Drug Administration (FDA) in 2005 for the treatment of metastatic breast cancer. The annual revenue of Abraxane is now 967 million \$, which testifies the size of the market for nanotherapeutics[110,111]. The research about polymer NPs as drug delivery systems is approximately 20 years more recent compared to that on liposomes. 
Consequently, despite the potential and market size for these formulations, only few examples can be found on the market. These include: i) Genexol-PM ${ }^{\circledR}$, a PEG-poly(D,L-lactide) PTX formulation approved in 2007 for breast cancer, ii) $\operatorname{Transdrug}^{\circledR}$, poly(isohexylcyanoacrylate) NPs loaded with Doxorubicin for the treatment of hepatocarcinoma, iii) Zinostatin Stimalamer ${ }^{\circledR}$ for the release of neocarzinostatin to hepatocellular carcinoma and iv) the paclitaxel formulation Paclical ${ }^{\circledR}$ for the treatment of ovarian cancer[76]. What it can be inferred from these few examples is that cancer treatment is dominant in the applications of polymer NPs for drug delivery. Indeed, cancer is the leading cause of death worldwide, thus justifying this huge research effort. Also, despite the numerous polymeric formulations developed on the bench, only very few of them were successful in reaching the market, with a success rate that is indeed very low. This is not only ascribable to the long route to achieve the commercialization approval from the regulatory agencies (i.e. FDA and European Medicines Agency, EMA), which could take more than 10 years, but also to the complexity in fulfilling the requirements along this route. The research groups involved in the development of new nanotherapeutics should therefore take advantage of the lessons learned from previous examples during the design stage, in order to avoid wasting time and money in proposing a formulation with a little chance of reaching the final goal of clinical approval. Therefore, to increase the success rate of polymeric formulations, one should understand the checkpoints on the road towards clinical translation.

First, the developed NPs should prove safe and effective in preliminary in vivo tests on at least two different animal models. This implies a detailed study of the pharmacokinetic of the formulation, as well as the evaluation of the possible insurgence of undesired side effects. In this sense, the publication of guidelines by the regulatory agencies or the development of a standardized procedure for this preliminary screening would be highly desirable. Ferrari et al. actually developed a method for the co-localization of both the polymer NPs as well as the payload when intravenously injected in a mouse model. 
This method relies on the chemical functionalization of the drug delivery system with a fluorophore followed by its loading with a metalorganic drug mimic compound. Following intravenous administration in mouse models, the authors were able to completely characterize the pharmacokinetic of both the NPs and the drug mimic compound via fluorescence imaging and inductively coupled plasma, respectively[112]. The information obtained from this analysis is useful not only to track the tissue accumulation of the formulation but also to study the individual fate of the NPs and the drug, which after administration present appreciable differences. Thus, this approach could serve as a roadmap for a preliminary screening of the therapeutic efficacy of a nanoformulation in the direction of a standardized procedure.

Also, in the preclinical assessment of a new nanopharmaceutical, the manufacturing method plays a key role. In particular, a scale up from few grams produced in the laboratory to several kilos on an industrial set-up is required. Therefore, reproducible, easily scalable processes following the good manufacturing practice (GMP) principles are an important prerequisite[113]. Strongly connected to this is the thorough characterization of the final product. In the case of polymer NPs, the therapeutic outcome is indeed related to the complex interactions of composition and microstructure. Therefore, it is essential to gain information about the molecular weight distribution, the size distribution through dynamic light scattering (DLS), the surface zeta-potential and the NP shape or morphology via microscopy techniques. From this detailed analysis, few key parameters should be considered as reporters for the formulation efficacy, in order to develop a robust quality control procedure. In this optic, the National Cancer Institute recently opened a subdivision, the Nanotechnology Characterization Laboratory (NCL), deputed to the development of guidelines for the characterization of nanopharmaceuticals at a preclinical stage[114]. This operates a screening of the developed formulations before the submission of an Investigational New Drug (IND) application, whose approval marks the entering in the clinical evaluation. 
This process follows the same route as for small molecule drugs. Therefore, the clinical evaluation consists of three phases aimed at assessing the safety, therapeutic efficacy and therapeutic relevance, respectively, of the investigated formulation, as shown in Table $\mathbf{1 .}$

Table 1. The steps followed by small molecules as well as NP-based therapeutics to reach the final approval and commercialization.

\begin{tabular}{|c|c|c|c|c|c|}
\hline \multirow[b]{3}{*}{$\begin{array}{c}\text { Parameter(s) } \\
\text { under } \\
\text { investigation }\end{array}$} & \multirow{3}{*}{$\begin{array}{l}\text { Pre-Clinical Stage } \\
\text { - Safety and Efficacy } \\
\text { (on mouse models) } \\
\text { - Scale up ability } \\
\text { - Full } \\
\text { characterization }\end{array}$} & \multicolumn{3}{|c|}{ Clinical Evaluation } & \multirow{2}{*}{$\begin{array}{c}\begin{array}{c}\text { After the } \\
\text { Approval }\end{array} \\
\text { Phase IV }\end{array}$} \\
\hline & & Phase I & Phase II & Phase III & \\
\hline & & Safety & $\begin{array}{l}\text { Therapeutic } \\
\text { efficacy }\end{array}$ & $\begin{array}{l}\text { Therapeutic } \\
\text { relevance }\end{array}$ & $\begin{array}{l}\bullet \text { Long-term } \\
\text { efficacy } \\
\bullet \text { Long-term } \\
\text { safety }\end{array}$ \\
\hline Time required & 5-10 years & $0.5-1.5$ years & $0.5-2$ years & $1-5$ years & $>2$ years \\
\hline
\end{tabular}

In particular, during the Phase $I$, the formulation under investigation is administered with a progressively increasing dosage to small populations of volunteers in order to assess the maximum tolerability. The most important goal of this phase is the evaluation of any dose-dependent adverse effect. Thus, the insurgence of premature side effects is the leading cause of failure at this stage[115, 116]. This stage takes up to 18 months but the success rate is pretty high, since $\sim 70 \%$ of the formulations are approved for the Phase II evaluation. At this stage, the main parameter under investigation is the therapeutic efficacy of the formulation. This means that the nanotherapeutics should provide unambiguous positive therapeutic outcomes after administration to patients. Specific guidelines are provided for the evaluation of this point. So for example in cancer treatment, a formulation is evaluated based on its ability to lead to a significant reduction in the tumor mass. The therapeutic outcome is obviously strictly dependent on the ability of the NPs to accumulate in the target, following either the EPR effect or an active targeting strategy. Therefore, a detailed pharmacokinetic study on human models is recommended to increase the chance of success in this stage, which is the most critical. In fact, only the $30 \%$ of the tested medicines are approved for entering the Phase III following the evidence of efficacy[117]. 
Finally, during the Phase III, the therapeutic relevance is evaluated. This means that the benefits/risk ratio is compared with that of standard treatments and conclusions on the justification for commercialization are drawn. In particular, the nanotherapeutic under investigation is administered to a population of patients and the outcome compared to that from a control group treated with an approved competitor or a placebo. After demonstration of therapeutic relevance, for $\sim 30 \%$ of formulations tested, a Marketing Authorization Application is submitted (in USA a New Drug Application is submitted to FDA). However, a formulation can be rejected even after commercialization. This happens if in the so-called Phase $I V$, the nanotherapeutic fails in demonstrating long-term safety and efficacy. It is clear that the road from the bench to the clinic requires time (up to 10 years), money and is highly risky. This makes the preclinical characterization even more important, with relevant studies aimed at assessing not only the safeness of a formulation but above all the therapeutic efficacy and relevance, being the Phase II and Phase III the bottle necks prior to clinical approval.

\section{Conclusions}

The field of nanomedicine is experiencing a rapid expansion in the last years, due to the benefits in terms of increased tolerability of the traditional drugs, more selective accumulation to the target tissue and possibility of achieving a controlled release. Also, the advent of novel therapeutics such as nucleic acids or proteins requires smart drug delivery systems preventing their premature degradation in the plasma environment as well as high rate of cell internalization for a positive therapeutic outcome[118, 119]. Polymer NPs represent a valuable tool to achieve these goals. In fact, the advent of the so-called living polymerization techniques paved the way to a strict control over the polymer microstructure. 
This is translated into a high controllability of the final physico-chemical properties of the NPs for the advantageous exploitation of the peculiar properties of the target tissue. In addition, polymer NPs allow the introduction of complex dynamics in response to environmental stimuli. This latter field is now attracting considerable attention as the most promising for a precise localization of the drug release in both space and time. Despite the high potential of polymer NPs as drug delivery vehicles, the road to their clinical application is long and highly insidious. This poses the fundamental problem of a thorough characterization of the formulation not only in terms of chemical and physical properties but also in terms of safety and therapeutic efficacy already at the pre-clinical stage, taking advantage of the lessons learned from previous cases of polymer NPs rejected during the clinical trials. This would enable the reduction of the risk and consequently to avoid wasting time and money for clinical studies. It is clear that this field is extremely variegate and expertise from chemistry, chemical engineering, pharmacy and biology should be gathered in an interdisciplinary team for the development of a polymer formulation with high chances of clinical translation. 


\section{References}

1. Langer, R., Drug delivery and targeting. Nature, 1998. 392(6679): p. 5-10.

2. Truong, N.P., et al., The importance of nanoparticle shape in cancer drug delivery. Expert Opinion on Drug Delivery, 2015. 12(1): p. 129-142.

3. Alonso, M.J. and N.S. Csaba, Nanostructured Biomaterials for Overcoming Biological Barriers, ed. M.J. Alonso and N.S. Csaba. 2012.

4. Saraiva, C., et al., Nanoparticle-mediated brain drug delivery: Overcoming blood-brain barrier to treat neurodegenerative diseases. Journal of Controlled Release, 2016. 235: p. 3447.

5. Jin, J.-f., et al., The optimal choice of medication administration route regarding intravenous, intramuscular, and subcutaneous injection. Patient Preference and Adherence, 2015. 9: p. 923-942.

6. Prabaharan, M., Chitosan-based nanoparticles for tumor-targeted drug delivery. International Journal of Biological Macromolecules, 2015. 72: p. 1313-1322.

7. Fan, Y.C. and J.J. Moon, Nanoparticle Drug Delivery Systems Designed to Improve Cancer Vaccines and Immunotherapy. Vaccines, 2015. 3(3): p. 662-685.

8. Estelrich, J., et al., Iron Oxide Nanoparticles for Magnetically-Guided and MagneticallyResponsive Drug Delivery. International Journal of Molecular Sciences, 2015. 16(4): p. 80708101.

9. Mou, X.B., et al., Applications of Magnetic Nanoparticles in Targeted Drug Delivery System. Journal of Nanoscience and Nanotechnology, 2015. 15(1): p. 54-62.

10. Bharti, C., et al., Mesoporous silica nanoparticles in target drug delivery system: A review. International Journal of Pharmaceutical Investigation, 2015. 5(3): p. 124-133.

11. Baeza, A., M. Colilla, and M. Vallet-Regi, Advances in mesoporous silica nanoparticles for targeted stimuli-responsive drug delivery. Expert Opinion on Drug Delivery, 2015. 12(2): p. 319-337. 
12. Choi, C.H.J., et al., Targeting kidney mesangium by nanoparticles of defined size. Proceedings of the National Academy of Sciences, 2011. 108(16): p. 6656-6661.

13. De Jong, W.H. and P.J.A. Borm, Drug delivery and nanoparticles: Applications and hazards. International Journal of Nanomedicine, 2008. 3(2): p. 133-149.

14. Nair, A.V., et al., Characterizing the Interactions of Organic Nanoparticles with Renal Epithelial Cells in Vivo. ACS Nano, 2015. 9(4): p. 3641-3653.

15. Wilczewska, A.Z., et al., Nanoparticles as Drug Delivery Systems. Pharmacological Reports, 2012. 64(5): p. 1020-1037.

16. Sadauskas, E., et al., Kupffer cells are central in the removal of nanoparticles from the organism. Particle and Fibre Toxicology, 2007. 4(1): p. 10.

17. Bertrand, N., et al., Mechanistic understanding of in vivo protein corona formation on polymeric nanoparticles and impact on pharmacokinetics. Nature Communications, 2017. 8: p. 8 .

18. Maiolo, D., et al., Nanomedicine delivery: does protein corona route to the target or off road? Nanomedicine, 2015. 10(21): p. 3231-3247.

19. Yang, Q. and S.K. Lai, Anti-PEG immunity: emergence, characteristics, and unaddressed questions. Wiley Interdisciplinary Reviews-Nanomedicine and Nanobiotechnology, 2015. 7(5): p. 655-677.

20. Suk, J.S., et al., PEGylation as a strategy for improving nanoparticle-based drug and gene delivery. Advanced Drug Delivery Reviews, 2016. 99: p. 28-51.

21. Mima, Y., et al., Anti-PEG IgM Is a Major Contributor to the Accelerated Blood Clearance of Polyethylene Glycol-Conjugated Protein. Molecular Pharmaceutics, 2015. 12(7): p. 24292435.

22. Grenier, P., et al., Anti-polyethylene glycol antibodies alter the protein corona deposited on nanoparticles and the physiological pathways regulating their fate in vivo. Journal of Controlled Release, 2018. 287: p. 121-131. 
23. Park, K., Impact of anti-PEG antibodies on PEGylated nanoparticles fate in vivo. Journal of Controlled Release, 2018. 287: p. 257-257.

24. Hojfeld, S.G., et al., Genetic predisposition to PEG-asparaginase hypersensitivity in children treated according to NOPHO ALL2008. British Journal of Haematology, 2019. 184(3): p. 405-417.

25. Krummenacher, M., et al., PEG 3350 ALLERGY PRESENTING AS DEPO-NISOLONE (R) ANAPHYLAXIS. Internal Medicine Journal, 2018. 48: p. 12-13.

26. Stone, C.A., et al., Immediate Hypersensitivity to Polyethylene Glycols and Polysorbates: More Common Than We Have Recognized. Journal of Allergy and Clinical Immunology-in Practice, 2019. 7(5): p. 1533-1540.

27. Eldar-Boock, A., et al., Integrin-targeted nano-sized polymeric systems for paclitaxel conjugation: a comparative study. Journal of Drug Targeting, 2017. 25(9-10): p. 829-844.

28. Kierstead, P.H., et al., The effect of polymer backbone chemistry on the induction of the accelerated blood clearance in polymer modified liposomes. Journal of Controlled Release, 2015. 213: p. 1-9.

29. Singh, J., et al., Polymer Drug Conjugates: Recent Advancements in Various Diseases. Current Pharmaceutical Design, 2016. 22(19): p. 2821-2843.

30. Sponchioni, M., et al., Poly(HPMA)-based copolymers with biodegradable side chains able to self assemble into nanoparticles. RSC Advances, 2017. 7(80): p. 50981-50992.

31. Amoozgar, Z. and Y. Yeo, Recent advances in stealth coating of nanoparticle drug delivery systems. Wiley Interdisciplinary Reviews: Nanomedicine and Nanobiotechnology, 2012. 4(2): p. 219-233.

32. Capasso Palmiero, U., et al., Zwitterionic Polyester-Based Nanoparticles with Tunable Size, Polymer Molecular Weight, and Degradation Time. Biomacromolecules, 2018. 19(4): p. $1314-1323$ 
33. $\mathrm{Xu}, \mathrm{X} . \mathrm{Y}$., et al., Cancer nanomedicine: from targeted delivery to combination therapy. Trends in Molecular Medicine, 2015. 21(4): p. 223-232.

34. Ladd, J., et al., Zwitterionic Polymers Exhibiting High Resistance to Nonspecific Protein Adsorption from Human Serum and Plasma. Biomacromolecules, 2008. 9(5): p. 1357-1361.

35. Li, Y., et al., Zwitterionic Poly(carboxybetaine)-based Cationic Liposomes for Effective Delivery of Small Interfering RNA Therapeutics without Accelerated Blood Clearance Phenomenon. Theranostics, 2015. 5(6): p. 583-596.

36. García, K.P., et al., Zwitterionic Coatings: Zwitterionic-Coated "Stealth" Nanoparticles for Biomedical Applications: Recent Advances in Countering Biomolecular Corona Formation and Uptake by the Mononuclear Phagocyte System (Small 13/2014). Small, 2014. 10(13): p. $2505-2505$.

37. Stylianopoulos, T. and R.K. Jain, Design considerations for nanotherapeutics in oncology. Nanomedicine-Nanotechnology Biology and Medicine, 2015. 11(8): p. 1893-1907.

38. Moscatelli, D. and M. Sponchioni, 12 - Bioresorbable polymer nanoparticles in the medical and pharmaceutical fields: A promising field, in Bioresorbable Polymers for Biomedical Applications, G. Perale and J. Hilborn, Editors. 2017, Woodhead Publishing. p. 265-283.

39. Asua, J.M. and H.A.S. Schoonbrood, Reactive surfactants in heterophase polymerization. Acta Polymerica, 1998. 49(12): p. 671-686.

40. Ferrari, R., et al., Synthesis of surfactant free PCL-PEG brushed nanoparticles with tunable degradation kinetics. International Journal of Pharmaceutics, 2013. 453(2): p. 551-559.

41. Ferrari, R., et al., epsilon-Caprolactone-Based Macromonomers Suitable for Biodegradable Nanoparticles Synthesis through Free Radical Polymerization. Macromolecules, 2011. 44(23): p. 9205-9212.

42. Yu, Y.C., et al., PLA-based nanoparticles with tunable hydrophobicity and degradation kinetics. Journal of Polymer Science Part a-Polymer Chemistry, 2012. 50(24): p. 5191-5200. 
43. Colombo, C., et al., PEGylated Nanoparticles Obtained through Emulsion Polymerization as Paclitaxel Carriers. Molecular Pharmaceutics, 2016. 13(1): p. 40-46.

44. Sitia, L., et al., Fate of PLA and PCL-Based Polymeric Nanocarriers in Cellular and Animal Models of Triple-Negative Breast Cancer. Biomacromolecules, 2016. 17(3): p. 744-755.

45. Hawker, C.J., A.W. Bosman, and E. Harth, New polymer synthesis by nitroxide mediated living radical polymerizations. Chemical Reviews, 2001. 101(12): p. 3661-3688.

46. Nicolas, J., et al., Nitroxide-mediated polymerization. Progress in Polymer Science, 2013. 38(1): p. 63-235.

47. Hadasha, W. and B. Klumperman, Atom transfer radical polymerization as a powerful tool in the synthesis of molecular brushes. Polymer International, 2014. 63(5): p. 824-834.

48. Siegwart, D.J., J.K. Oh, and K. Matyjaszewski, ATRP in the design of functional materials for biomedical applications. Progress in Polymer Science, 2012. 37(1): p. 18-37.

49. Stenzel, M.H., RAFT polymerization: an avenue to functional polymeric micelles for drug delivery. Chemical Communications, 2008(30): p. 3486-3503.

50. Moad, G., E. Rizzardo, and S.H. Thang, Living radical polymerization by the RAFT process. Australian Journal of Chemistry, 2005. 58(6): p. 379-410.

51. Moad, G., E. Rizzardo, and S.H. Thang, Radical addition-fragmentation chemistry in polymer synthesis. Polymer, 2008. 49(5): p. 1079-1131.

52. Chong, Y.K., et al., A more versatile route to block copolymers and other polymers of complex architecture by living radical polymerization: The RAFT process. Macromolecules, 1999. 32(6): p. 2071-2074.

53. Palmiero, U.C., et al., Strategies to combine ROP with ATRP or RAFT polymerization for the synthesis of biodegradable polymeric nanoparticles for biomedical applications. Polymer Chemistry, 2018. 9(30): p. 4084-4099. 
54. Palmiero, U.C., et al., RAFT Macro-Surfmers and Their Use in the ab Initio RAFT Emulsion Polymerization To Decouple Nanoparticle Size and Polymer Molecular Weight. Macromolecules, 2016. 49(22): p. 8387-8396.

55. Capasso Palmiero, U., et al., Readily prepared biodegradable nanoparticles to formulate poorly water soluble drugs improving their pharmacological properties: The example of trabectedin. Journal of Controlled Release, 2018. 276: p. 140-149.

56. Gordon, E.M., et al., Trabectedin for Soft Tissue Sarcoma: Current Status and Future Perspectives. Advances in Therapy, 2016. 33(7): p. 1055-1071.

57. Newman, D.J., Developing natural product drugs: Supply problems and how they have been overcome. Pharmacology \& Therapeutics, 2016. 162: p. 1-9.

58. Sessa, C., et al., Trabectedin for Women With Ovarian Carcinoma After Treatment With Platinum and Taxanes Fails. Journal of Clinical Oncology, 2005. 23(9): p. 1867-1874.

59. Baum, D.C., et al., Mutagenesis and Oncogenesis by Chromosomal Insertion of Gene Transfer Vectors. Human Gene Therapy, 2006. 17(3): p. 253-263.

60. Green, J.J., R. Langer, and D.G. Anderson, A combinatorial polymer library approach yields insight into nonviral gene delivery. Accounts of Chemical Research, 2008. 41(6): p. 749-759.

61. Yin, H., et al., Non-viral vectors for gene-based therapy. Nature Reviews Genetics, 2014. 15 : p. 541.

62. Kunjachan, S., et al., Passive versus Active Tumor Targeting Using RGD-and NGR-Modified Polymeric Nanomedicines. Nano Letters, 2014. 14(2): p. 972-981.

63. Maeda, H., G.Y. Bharate, and J. Daruwalla, Polymeric drugs for efficient tumor-targeted drug delivery based on EPR-effect. European Journal of Pharmaceutics and Biopharmaceutics, 2009. 71(3): p. 409-419.

64. Chauhan, V.P., et al., Normalization of tumour blood vessels improves the delivery of nanomedicines in a size-dependent manner. Nature Nanotechnology, 2012. 7(6): p. 383-388. 
65. Cabral, H., et al., Accumulation of sub-100 $\mathrm{nm}$ polymeric micelles in poorly permeable tumours depends on size. Nature Nanotechnology, 2011. 6(12): p. 815-823.

66. Stylianopoulos, T., EPR-effect: utilizing size-dependent nanoparticle delivery to solid tumors. Therapeutic Delivery, 2013. 4(4): p. 421-423.

67. Nichols, J.W. and Y.H. Bae, EPR: Evidence and fallacy. Journal of Controlled Release, 2014. 190: p. 451-464.

68. Sparreboom, A., et al., Comparative preclinical and clinical pharmacokinetics of a cremophor-free, nanoparticle albumin-bound paclitaxel (ABI-007) and paclitaxel formulated in cremophor (Taxol). Clinical Cancer Research, 2005. 11(11): p. 4136-4143.

69. Danhier, F., To exploit the tumor microenvironment: Since the EPR effect fails in the clinic, what is the future of nanomedicine? Journal of Controlled Release, 2016. 244: p. 108-121.

70. Brannon-Peppas, L. and J.O. Blanchette, Nanoparticle and targeted systems for cancer therapy. Advanced Drug Delivery Reviews, 2012. 64, Supplement: p. 206-212.

71. Danielli, R., et al., Armed antibodies for cancer treatment: a promising tool in a changing era. Cancer Immunology, Immunotherapy, 2015. 64(1): p. 113-121.

72. Fernandez, M., F. Javaid, and V. Chudasama, Advances in targeting the folate receptor in the treatment/imaging of cancers. Chemical Science, 2018. 9(4): p. 790-810.

73. Mc Carron, P., et al., Targeting the Folate Receptor: Improving Efficacy in Inorganic Medicinal Chemistry. Current Medicinal Chemistry, 2018. 25(23): p. 2675-2708.

74. Muhamad, N., T. Plengsuriyakarn, and K. Na-Bangchang, Application of active targeting nanoparticle delivery system for chemotherapeutic drugs and traditional/herbal medicines in cancer therapy: a systematic review. International Journal of Nanomedicine, 2018. 13: p. 3921-3935.

75. Poltavets, Y.I., et al., In vitro anticancer activity of folate-modified docetaxel-loaded PLGA nanoparticles against drug-sensitive and multidrug-resistant cancer cells. Cancer Nanotechnology, 2019. 10: p. 17. 
76. Ferrari, R., et al., Polymer nanoparticles for the intravenous delivery of anticancer drugs: the checkpoints on the road from the synthesis to clinical translation. Nanoscale, 2018. 10(48): p. 22701-22719.

77. Alarcon, C.D.H., S. Pennadam, and C. Alexander, Stimuli responsive polymers for biomedical applications. Chemical Society Reviews, 2005. 34(3): p. 276-285.

78. Evans, D.F., et al., Measurement of gastrointestinal pH profiles in normal ambulant human subjects. Gut, 1988. 29(8): p. 1035.

79. Koziolek, M., et al., Investigation of $\mathrm{pH}$ and Temperature Profiles in the GI Tract of Fasted Human Subjects Using the Intellicap((R)) System. Journal of Pharmaceutical Sciences, 2015. 104(9): p. 2855-2863.

80. Ibarra-Montano, E.L., et al., Determination of $p K a$ Values for Acrylic, Methacrylic and Itaconic Acids by ${ }^{1} \mathrm{H}$ and ${ }^{13} \mathrm{C} N \mathrm{NM}$ in Deuterated Water. Journal of Applied Solution Chemistry and Modeling, 2015. 4: p. 7-18.

81. Ferreira, I.S., et al., Improvement of the antibacterial activity of daptomycin-loaded polymeric microparticles by Eudragit RL 100: an assessment by isothermal microcalorimetry. International journal of pharmaceutics, 2015. 485(1-2): p. 171-182.

82. Higashi, K., et al., The effect of drug and EUDRAGIT® $S 100$ miscibility in solid dispersions on the drug and polymer dissolution rate. International journal of pharmaceutics, 2015. 494(1): p. 9-16.

83. Jablan, J. and M. Jug, Development of Eudragit ${ }^{\circledR}$ S100 based pH-responsive microspheres of zaleplon by spray-drying: Tailoring the drug release properties. Powder technology, 2015. 283: p. 334-343.

84. Feng, L.Z., et al., The acidic tumor microenvironment: a target for smart cancer nanotheranostics. National Science Review, 2018. 5(2): p. 269-286. 
85. Wu, W., et al., Endogenous pH-responsive nanoparticles with programmable size changes for targeted tumor therapy and imaging applications. Theranostics, 2018. 8(11): p. 30383058.

86. Ma, X., et al., Ultra-pH-Sensitive Nanoprobe Library with Broad pH Tunability and Fluorescence Emissions. Journal of the American Chemical Society, 2014. 136(31): p. 1108511092.

87. Wang, C., et al., Investigation of endosome and lysosome biology by ultra pH-sensitive nanoprobes. Advanced Drug Delivery Reviews, 2017. 113: p. 87-96.

88. Wang, Y., et al., A nanoparticle-based strategy for the imaging of a broad range of tumours by nonlinear amplification of microenvironment signals. Nature Materials, 2013. 13: p. 204.

89. Zhao, T., et al., A transistor-like pH nanoprobe for tumour detection and image-guided surgery. Nature Biomedical Engineering, 2016. 1: p. 0006.

90. Lale, S.V., et al., Multifunctional ATRP based $p H$ responsive polymeric nanoparticles for improved doxorubicin chemotherapy in breast cancer by proton sponge effect/endo-lysosomal escape. Polymer Chemistry, 2015. 6(11): p. 2115-2132.

91. Nel, A.E., et al., Understanding biophysicochemical interactions at the nano-bio interface. Nat Mater, 2009. 8(7): p. 543-557.

92. Zhu, X.J., et al., Temperature-feedback upconversion nanocomposite for accurate photothermal therapy at facile temperature. Nature Communications, 2016. 7: p. 10.

93. Sponchioni, M., U. Capasso Palmiero, and D. Moscatelli, Thermo-responsive polymers: Applications of smart materials in drug delivery and tissue engineering. Materials Science and Engineering: C, 2019. 102: p. 589-605.

94. Zhang, Q., et al., Thermoresponsive polymers with lower critical solution temperature: from fundamental aspects and measuring techniques to recommended turbidimetry conditions. Materials Horizons, 2017. 4(2): p. 109-116. 
95. Halperin, A., M. Kröger, and F.M. Winnik, Poly(N-isopropylacrylamide) Phase Diagrams: Fifty Years of Research. Angewandte Chemie International Edition, 2015. 54(51): p. 1534215367.

96. Lutz, J.F., O. Akdemir, and A. Hoth, Point by point comparison of two thermosensitive polymers exhibiting a similar LCST: Is the age of poly(NIPAM) over? Journal of the American Chemical Society, 2006. 128(40): p. 13046-13047.

97. Hoogenboom, R. and H. Schlaad, Thermoresponsive poly(2-oxazoline)s, polypeptoids, and polypeptides. Polymer Chemistry, 2017. 8(1): p. 24-40.

98. Weber, C., R. Hoogenboom, and U.S. Schubert, Temperature responsive bio-compatible polymers based on poly(ethylene oxide) and poly(2-oxazoline)s. Progress in Polymer Science, 2012. 37(5): p. 686-714.

99. Cortez-Lemus, N.A. and A. Licea-Claverie, Poly(N-vinylcaprolactam), a comprehensive review on a thermoresponsive polymer becoming popular. Progress in Polymer Science, 2016. 53: p. 1-51.

100. Liu, J., et al., Poly(N-vinylcaprolactam): A Thermoresponsive Macromolecule with Promising Future in Biomedical Field. Advanced Healthcare Materials, 2014. 3(12): p. 19411968.

101. Lutz, J.F., Polymerization of oligo(ethylene glycol) (meth)acrylates: Toward new generations of smart biocompatible materials. Journal of Polymer Science Part a-Polymer Chemistry, 2008. 46(11): p. 3459-3470.

102. Lutz, J.F., A. Hoth, and K. Schade, Design of Oligo(ethylene glycol)-Based Thermoresponsive Polymers: an Optimization Study. Designed Monomers and Polymers, 2009. 12(4): p. 343353.

103. Chung, J.E., et al., Thermo-responsive drug delivery from polymeric micelles constructed using block copolymers of poly(N-isopropylacrylamide) and poly(butylmethacrylate). Journal of Controlled Release, 1999. 62: p. 115-127. 
104. Sponchioni, M., et al., RAFT copolymerization of oppositely charged monomers and its use to tailor the composition of nonfouling polyampholytes with an UCST behaviour. Reaction Chemistry \& Engineering, 2019. 4(2): p. 436-446.

105. Sponchioni, M., et al., Influence of the Polymer Structure Over Self-Assembly and ThermoResponsive Properties: The Case of PEG-b-PCL Grafted Copolymers via a Combination of RAFT and ROP. Journal of Polymer Science Part a-Polymer Chemistry, 2016. 54(18): p. 2919-2931.

106. Seuring, J. and S. Agarwal, Polymers with Upper Critical Solution Temperature in Aqueous Solution. Macromolecular Rapid Communications, 2012. 33(22): p. 1898-1920.

107. Li, W., et al., Antitumor drug delivery modulated by a polymeric micelle with an upper critical solution temperature. Angewandte Chemie International Edition, 2015. 54(10): p. 3126-3131.

108. Ding, C.D., et al., Recent Advances in Stimuli-Responsive Release Function Drug Delivery Systems for Tumor Treatment. Molecules, 2016. 21(12): p. 30.

109. Cavallaro, G., et al., Reversibly stable thiopolyplexes for intracellular delivery of genes. Journal of Controlled Release, 2006. 115(3): p. 322-334.

110. Bernabeu, E., et al., Paclitaxel: What has been done and the challenges remain ahead. International Journal of Pharmaceutics, 2017. 526(1-2): p. 474-495.

111. Kratz, F., A clinical update of using albumin as a drug vehicle - A commentary. Journal of Controlled Release, 2014. 190: p. 331-336.

112. Ferrari, R., et al., Biocompatible Polymer Nanoformulation To Improve the Release and Safety of a Drug Mimic Molecule Detectable via ICP-MS. Molecular Pharmaceutics, 2017. 14(1): p. 124-134.

113. Operti, M.C., et al., A comparative assessment of continuous production techniques to generate sub-micron size PLGA particles. International Journal of Pharmaceutics, 2018. 550(1-2): p. 140-148. 
114. Siegrist, S., et al., Preclinical hazard evaluation strategy for nanomedicines. Nanotoxicology, 2019. 13(1): p. 73-99.

115. Von Hoff, D.D., et al., Phase I Study of PSMA-Targeted Docetaxel-Containing Nanoparticle BIND-014 in Patients with Advanced Solid Tumors. Clinical Cancer Research, 2016. 22(13): p. 3157.

116. Tyagi, P. and J.L. Santos, Macromolecule nanotherapeutics: approaches and challenges. Drug Discovery Today, 2018. 23(5): p. 1053-1061.

117. Li, Z., et al., Cancer drug delivery in the nano era: An overview and perspectives (Review). Oncology Reports, 2017. 38(2): p. 611-624.

118. Davis, M.E., The First Targeted Delivery of siRNA in Humans via a Self-Assembling, Cyclodextrin Polymer-Based Nanoparticle: From Concept to Clinic. Molecular Pharmaceutics, 2009. 6(3): p. 659-668.

119. Zuckerman, J.E. and M.E. Davis, Clinical experiences with systemically administered siRNAbased therapeutics in cancer. Nature Reviews Drug Discovery, 2015. 14(12): p. 843-856. 


\title{
Chapter 2. Extracellular Vesicles in Regenerative Medicine
}

Miriam Romano ${ }^{1,2}$, Andrea Zendrini ${ }^{3}$, Lucia Paolini ${ }^{1,2}$, Sara Busatto ${ }^{4}$, Anna C. Berardi ${ }^{5}$, Paolo Bergese $^{1,2}$ and Annalisa Radeghieri ${ }^{1,2^{*}}$

1. Department of Molecular and Translational Medicine, University of Brescia, 25123 Brescia, Italy

2. CSGI, Research Center for Colloids and Nanoscience, 50019 Florence, Italy

3. Department of Animal Science, Food and Nutrition - Università Cattolica del Sacro Cuore, 29122 Piacenza, Italy

4. Department of Transplantation Medicine; Department of Physiology and Biomedical Engineering, Mayo Clinic, Jacksonville, FL 32224, USA

5. U.O.C. of Immunohematology and Transfusion Medicine, Laboratory of Stem Cells, Spirito Santo Hospital, Pescara, Italy

*annalisa.radeghieri@unibs.it

\begin{abstract}
Regenerative medicine is a multidisciplinary field aimed at developing methods, molecular agents and (nano)materials to regrow, repair or replace damaged, malfunctioning or missing tissues. Current approaches include and combine use of stem cells, tissue engineering based on functional biodegradable scaffolds and cell-free strategies, with stem cells and their progenitors playing the main role. However, it is now recognized that the therapeutic efficacy of stem cells largely depends on paracrine secreted soluble factors and extracellular vesicles (EVs). Preclinical and clinical studies indicate that EVs can exert immuno-modulatory and regenerative action, thus efficiently recapitulating the therapeutic effects of stem cells. On the other hand, EVs are uncapable of selfreplication agents and can be non-immunogenic, thus offering remarkable advantages and safety over stem cells for therapeutic translation. This chapter, after a friendly introduction of EV biological and physicochemical properties, will present and discuss advances in EV-based regenerative medicine.
\end{abstract}

Keywords: Extracellular vesicles, Exosomes, Microvesicles, Regeneration, Tissue engineering, Immunomodulation, Mesenchymal Stem Cells, Translational medicine, Nanomedicine 


\section{Introduction}

For decades researchers have dreamed of replicating a fascinating natural phenomenon: the regeneration of body parts ${ }^{1}$. Regenerative medicine is the branch of medicine which seeks to develop strategies to reproduce abilities found in nature and may be defined as "the process of regenerating, or replacing, cells, tissues or organs to establish, or restore, normal function of the damaged tissue or organ"2.

Over the last three decades, new promising regenerative therapeutic strategies based on the design of novel molecular agents and (nano)materials have been explored ${ }^{3}$.

All strategies under study and/or under clinical investigation can be grouped in three major sets, namely (1) cell-based therapies, (2) tissue engineering and (3) cell-free therapies (Fig.2.1). Among the most recently explored cell-free therapies, extracellular vesicles (EVs), together with lipoproteins, are the biogenic newcomers in the field, which also comprises synthetic nanoparticles, such as liposomes and inorganic nanomaterials (e.g. gold nanoparticles) (Fig. 2.1). EVs are cellderived nanoparticles delimited by a lipid membrane that protects lumen-located bioactive molecules, mainly proteins and nucleic acids ${ }^{4}$. EVs are one of the main mediators of long-distance intercellular communication processes. In fact, through the delivery of their cargo, EV can deeply modulate the biological functions of the target cells regulating both physiological and pathological processes ${ }^{4}$.

Given their ability to transport biologically active molecules, EVs are increasingly being explored in the field of regenerative medicine.

This chapter will give a concise introduction of the two leading techniques in the field, namely cellbased therapies (Section 2) and tissue engineering (Section 3). Section 4 will provide an overview of the most promising non-EV cell-free therapies. Lastly, the two conclusive sections of the chapter will talk about EV biological and physicochemical properties (Section 5) and then discuss their potential role in regenerative medicine according the state-of-the-art literature (Section 6).

$* * *$ Insert Figure $2.1 * * *$

Figure 2.1 Regenerative Medicine Panorama: a schematic overview of some of the regenerative medicine strategies and therapeutics that are currently subject of study and/or under clinical investigation. Abbreviations: MSCs, Mesenchymal Stem Cells; iPS, induced Pluripotent Stem Cells; UC-MSCs, Umbilical Cord-derived MSCs. 


\section{Cell-based therapies}

Stem cells (SCs) contributed to remarkable advancements in regenerative medicine, promoting and facilitating clinical translation into Stem-Cell based therapeutics ${ }^{5}$. To date, various native or engineered SC types have been studied for clinical applications such as embryonic SCS (ESCS), induced pluripotent SCs (iPSCs) or adult SCs. In particular, Hematopoietic SCs (HSCs), Mesenchymal SCs (MSCs) and Neuronal SCs (NSCS) have been widely explored ${ }^{6}$. A number of studies indicate MSCs are the ideal choice for certain cell-based therapies, due to their easy accessibility, genomic stability, immunomodulatory properties and few ethical issues ${ }^{7}$.

Cells derived from bone-marrow and peripheral blood have been applied in the field of hematological malignancies for over 60 years, while other stem cell-therapies are limited by many critical issues arising from culturing conditions and transplantation ${ }^{8}$. In fact, certain types of cells must be amplified in vitro before transplantation to obtain sufficient numbers which can potentially cause damage from oxidative, or mechanical, stress, and possible cell-mutation, chromosome abnormality, senescence or infection ${ }^{9}$. Moreover, the efficiency of cell therapy approaches depends also on other critical points not yet resolved such as the high rate of cellular death that occurs during cell transplantation, known as anoikis (anchorage-dependent cell death), and the activation of the host immune response ${ }^{10}$. Furthermore, cell-therapy protocols must be rigorously evaluated by qualified regulatory bodies to guarantee product standards ${ }^{11}$.

\section{Tissue engineering}

Evolution in the therapeutic regenerative approaches has led to the development of tissue engineering (TE) in which scaffolds, combined with cells and/or biologically active molecules form functional tissues and even whole organs ${ }^{12}$. The characteristics of the TE prototype depend on the interaction of three components:

1) the autologous or xenogenic cells that will form the tissue;

2) the synthetic, or natural biomaterial, scaffolds which mimics the physical form of the tissue, holding the cell together;

3) the biological-signaling molecules, such as growth factors, which stimulate a specific phenotype in cell expression.

Further, TE-scaffolds, that mimic the extracellular matrix, have also been fabricated to improve cellculturing, recreating native microenvironments ${ }^{13,14}$. 
Scaffolds can be also used as localized delivery systems themselves, both providing a controlled release of bioactive molecules (e.g. growth factors ${ }^{13}$ or nucleic acids ${ }^{15}$ ) and preventing undesirable immune response events, thus potentially improving the renewal of tissues with limited regenerative capacities ${ }^{13,16}$.

Safety and biocompatibility issues have yet to be resolved in order to predict long-term human-body response after cell-biomaterial transplantation ${ }^{12}$.

\section{Cell-free therapies}

The translation of cell-based therapies is mainly limited by the pitfalls described in Section 2 . Therefore, different cell-free approaches have been attempted as an alternative or complementary route. In this section, the most studied cell-free approaches will be briefly described, including the use of soluble factors and nanosized particles.

\subsection{Soluble factors}

Recent data demonstrate that the regenerative potential of MSCs is mainly related to their secretome, a set of soluble (SFs) or EV-encapsulated bioactive factors released from MSCs, which exert paracrine effects on neighbouring cells and tissues ${ }^{17,18}$. Mitchell and colleagues ${ }^{19}$ showed that Adipose-derived SC (ADSC)-secretome promoted muscle regeneration both in vitro and in vivo. Experiments indicate that SFs and EV fractions acted synergistically in promoting and modulating tissue restoration.

Long before discovering the existence and the promising properties of EVs (that will be discussed extensively in Sections 5 and 6), researchers had started to focus their efforts in order to understand the role and the possible exploitation of SFs in the regenerative process. Among those, immunomodulatory and pro-angiogenic proteins ${ }^{20}$ have been widely studied. Several membranebound proteins involved in inflammatory response, such as inteleukin-6 receptor (IL-6R), can be released by cells in a soluble form, thus playing as molecular mediators of regeneration ${ }^{21}$. Cytokines play a pivotal role in different stages of bone restoration, promoting resident Bone Marrow-MSCs (BM-MSCs) recruitment to the damaged area, where BM-MSCs start to proliferate and differentiate in mature bone cells ${ }^{22}$. Due to the involvement of cytokines and chemokines in SC homing and recruitment, their effects are being investigated both in bone and in cutaneous wound regeneration. For instance, it has been demonstrated that Interleukin-3 (IL-3) treatment promotes MSC differentiation into osteoblasts and increases in vitro and in vivo motility and wound healing 
abilities of MSCs, preventing bone and cartilage damage ${ }^{23}$. Likewise, treatment with conditioned media from ADSCS stimulated with tumour necrosis factor- $\alpha$ (TNF- $\alpha$ ), a proinflammatory cytokine, accelerated wound closure, angiogenesis, proliferation and immune cell infiltration into skin wound in vivo ${ }^{24}$. MSCs secrete also trophic factors that trigger target cell response through the binding and the activation of cell receptors. In specific conditions, e.g. after tissue injury, these factors can be also captured by the extracellular matrix acting in a paracrine manner ${ }^{25}$. Growth factors (GFs) released from MSCs comprise transforming growth factor (TGF) $\beta$, Epidermal Growth Factor (EGF) and Insulin Growth Factor -1 (IGF-1) $)^{26}$ as well as Bone Morphogenetic Factor (BMP), Fibroblast Growth Factor (FGF) $)^{27}$ and many others ${ }^{28}$.

Although SF therapeutic potential holds great promise in regenerative medicine applications, translation of SF into clinical treatments has been hindered by limitations including poor protein stability, low recombinant expression yield, and suboptimal efficacy ${ }^{25}$.

Nevertheless, some growth factors have been clinically-approved such as Regranex ${ }^{\circledR}$ (a plateletderived growth factor (PDGF)-BB) for the treatment of diabetic neuropathic ulcers or Bone Morphogenetic Factors -2 and -7 (BMP-2 and BMP-7) for lumbar spine fusion and tibial fracture (InFUSE ${ }^{\text {TM }}$ Bone Graft/LT-Cage ${ }^{\mathrm{Tm}}$ ). Most of these products are based on engineered proteins, which present high stability, long serum half-life, efficient biodistribution and limited tissue diffusion ${ }^{25}$. However, side effects from systemic administration of GFs in high and repeated doses limit their application in regenerative medicine as standard-of-care therapy. Moreover, although GFs or immunomodulatory molecules can be delivered to injured areas through circulating cells or scaffold proteins ${ }^{21}$, they do not intrinsically present targeting properties and induce controversial reactions, e.g. ectopic tissue formation. The development of delivery systems, such as biocompatible scaffolds or biogenic nanoparticles might increase SF-based approaches.

\subsection{Biogenic and synthetic nanoparticles}

Both biogenic and synthetic nanoparticles have gained attention in the field of regenerative medicine. The term biogenic is referred to particles naturally secreted by cells or present in biological fluids, like EVs (which will be discussed in Section 5) or lipoproteins. Contrarily, biomimetic (e.g. liposomes) and inorganic nanoparticles (e.g. gold nanoparticles) are considered synthetic or fully artificial particles. Lipoproteins are composed by a hydrophobic core bounded by free cholesterol, phospholipids and proteins. Lipoproteins are highly heterogeneous in both size and density and are divided into five major groups: 1) high-density lipoproteins (HDLs), 2) low- 
density lipoproteins (LDLs), 3) intermediate-density lipoproteins (IDLs), 4) very low-density lipoproteins (VLDLs) and 5) chylomicrons. Among these five classes, HDLs are the most characterized and their effects on stem cell proliferation ${ }^{29}$, wound repair and angiogenesis ${ }^{30}$, and liver homeostasis ${ }^{31}$ are established. Liposomes (self-assembled vesicles) and inorganic nanoparticles have emerged as drug delivery systems combined with bioscaffolds. In fact, applications of liposomes have been investigated as slow-delivery system in cartilage repair ${ }^{32}$ and stem cell proliferation ${ }^{33}$. Likewise, same reports pointed out the possibility to use gold nanoparticles (AuNPs) as powerful delivery system in bone regeneration, promoting stem cell osteogenic differentiation ${ }^{34,35}$.

\section{Extracellular Vesicles (EVs) in a nutshell}

\subsection{EV biological and physicochemical properties}

In 1981 Trams et al. ${ }^{36}$ reported for the first time that normal and neoplastic cell lines release membrane-derived vesicles, later named EVs, hypothetically involved in cell-to-cell recognition or transport processes. By this discovery, several groups became interested in studying EVs, biological nanoparticles heterogeneous in size, membrane composition, and bioactive content, secreted by both eukaryotic and prokaryotic cells ${ }^{37}$. In 2006, Ratajczak et al. ${ }^{38}$ demonstrated that EVs isolated from Embryonic Stem Cells (ESCs) mediated horizontal RNA and protein transfer to Hematopoietic Progenitor Cells (HPCs), corroborating the hypothesis according to which EVs represent an alternative intercellular communication pathway.

EV biogenesis and uptake processes. To date, EVs are described as soft bio-nanoparticles that are "naturally released from the cell [...] and cannot replicate, i.e. do not contain a functional nucleus" 39 . Interestingly, EVs can be secreted following at least two biogenesis pathways. In particular, shedding vesicles (ectosomes or microvesicles) are secreted through outside plasma membrane budding, whereas intraluminal vesicles (ILVs) are produced through reverse membrane budding of the Multivesicular Bodies (MVBs) and released upon the fusion of MVB with the plasma membrane (exosomes) ${ }^{40}$. ILV formation is regulated by different independent and parallel pathways: 1) recruitment of the endosomal sorting complex required for transport (ESCRT) and reversible ubiquitylation of protein cargos, 2) synthesis of ceramide by neutral sphingomyelinase $(\mathrm{N} \text {-SMase })^{41}$ and 3 ) tetraspanins-dependent mechanism ${ }^{42}$. Exosome releasing is related to the activation of different RAB GTPases (RAS-related proteins) and SNARE complex proteins ${ }^{41}$. Instead, microvesicle shedding/budding has been less characterized, but it is known that it occurs after a 
localized rearrangement of the actin cytoskeleton, reorganization of the plasma membrane and activation of actin-myosin machinery ${ }^{43}$.

Independently of their biogenesis, vesicles are internalized by the target cell via different uptake mechanisms: endocytic pathways (e.g. receptor-mediated endocytosis, clathrin-dependent endocytosis, caveolin mediated endocytosis, phagocytosis, macropinocytosis, lipid raft mediated internalization ${ }^{44,45}$ or antigen presentation ${ }^{41}$ ) or fusion with the plasma membrane ${ }^{41}$. It is possible that a population of EVs can simultaneously trigger a number of different gateways into a cell, with the primary entry points depending on the cell type and EV constituents ${ }^{46,47}$.

EV molecular composition. EVs possess a dual nature: they are composed of an aqueous core enriched in soluble proteins and nucleic acids and a lipid membrane (where hydrophobic and amphiphilic moieties harbor, e.g proteins), which enclose and protect EV payload from extracellular environment (Fig. 2.2). Proteomic analysis show that EVs contain a specific subset of cellular proteins, including transmembrane (e.g. tetraspanins CD81/CD9/CD63 and integrins) and GPIanchored proteins (e.g. CD73), depending on the secreting cell type ${ }^{39}$. Results of these studies are assembled into two major databases named Vesiclepedia (http://microvesicles.org/) and EVpedia (http://evpedia.info). Furthermore, current knowledge on EV molecular composition shows that EVs are enriched in specific lipid classes (e.g. phosphatidylserine) over than cells following an asymmetric distribution, as reported in Skotland et al. ${ }^{48}$. Interestingly, the great amount of saturated lipids and cholesterol contributes to EV membrane rigidity and therefore their stability as carriers of biologically active molecules ${ }^{49}$.

EVs contain in their aqueous core a great variety of nucleic acids, e.g. mRNAs ${ }^{50}$, miRNAs, isomiRs, snoRNA $^{51}$, IncRNAs ${ }^{52}$ and DNA (although depending on the subpopulation analyzed ${ }^{53,54}$ ). Functional transfers of genetic materials via vesicles have been demonstrated: miRNAs delivered by EVs can condition gene expression in distant cells ${ }^{4}$.

$* * *$ Insert Figure 2.2***

Figure 2.2 EV molecular composition. Examples of lipids, proteins and nucleic acids found in EVs. Abbreviations: SM, sphingomyelin; PE, phosphatidylethanolamine; PC, phosphatidylcholine; PE-O, alkyl-ether substituted phosphatidyl-ethanolamine; PS, phosphatidylserine; CHOL, cholesterol. For further information refer to ${ }^{4}$ and ${ }^{48}$. 
EV physicochemical properties. EVs are found in almost every bodily fluid, and their diameter spans three orders of magnitude - being the smallest particles detected around $30 \mathrm{~nm}$ in size, while the biggest exceed $1000 \mathrm{~nm}$.

EVs are generally reported as spherical particles, although ellipsoidal, tubular ${ }^{55}$ and more complex structures, including multilamellar vesicles ${ }^{56}$, are reported. EVs can be classified by their physical characteristics namely size, density, particle content, surface charge, and mechanical properties (Table 2.1). A general classification of EV subtypes according to their size and density have been proposed $^{39}$. They are mainly grouped into small EVs (sEVs) with a diameter $<100 \mathrm{~nm}$ or $<200 \mathrm{~nm}$ and medium/large EVs (m/I EVs) ranging in size more than $200 \mathrm{~nm}$, and into low-density EVs (IEVs) $\left(1.12-1.20 \mathrm{~g} \cdot \mathrm{cm}^{-3}\right)$ and high-density EVs (hEVs) $\left(1.25-1.30 \mathrm{~g} \cdot \mathrm{cm}^{-3}\right)^{55}$, showing distinct protein, lipid and nucleic acid content ${ }^{57}$.

Determination of EV particle number/concentration represent another important biophysical parameter, useful to compare different EV populations ${ }^{58}$ and to discriminate between physiological and pathological samples ${ }^{59}$. For example, EV plasma concentration in healthy donors is $\sim 1010 / \mathrm{mL}$ 60 , while the concentration of culture media-derived EVs is strictly dependent upon the cellular sources, as reported by Lane and colleagues ${ }^{61}$. Here, five different glioblastoma cell lines showed distinct EV particle content, ranging from 59.9 particles $/ \mathrm{mL}$ up to 259.2 particles/mL (U87 and G166 cell lines, respectively). However, EV quantification data are also hindered by the isolation protocol and the quantification method used ${ }^{62}$.

Considering their colloidal properties, EV surface charge is reflected by their zeta potential ( $\zeta-$ potential), a measure of charge stability that regulates particle-particle interactions. EV surface charge is mildly negative and varies together with the source of EVs: for instance, plasma EVs are generally more positive $(\sim-17 \mathrm{mV})$ than cerebrospinal fluid EVs $(\sim-30 \mathrm{mV})^{63}$. Overall negative charge is ascribable to EV membrane composition, which is enriched in glycolipids (e.g. gangliosides and modified ceramides ${ }^{48}$ ) and further tailored with many different types of glycans and glycoproteins. This fine-tuned decoration, together with peculiar structure, gives EVs remarkable delivery and targeting features.

In addition, the ability to properly carry molecular signals largely depends on the $\mathrm{pH}$, on the ionic strength of the biological fluid ${ }^{64}$ and on their mechanical properties, which are determined by EV membrane and intraluminal compositions ${ }^{65}$. Indeed, natural EV membrane buckling seems to be strictly related to EV membrane protein composition that could lead to a nonzero shear modulus ${ }^{65,66}$, affecting EV stability and functionality. For example, the Young's modulus of small EVs 
( $100 \mathrm{~nm}$ ) was seen to be around $200-300 \mathrm{MPa}$, much higher than one of synthetic lipid vesicles, pointing out an important structural role of membrane bounded proteins in the mechanical response of EVs. In this context, Vorselen et al. ${ }^{65}$ reported that EVs derived from red blood cells of patients with hereditary spherocytosis showed an altered protein composition and a significantly softer membrane compared to EVs from healthy subjects. Whitehead and colleagues ${ }^{67}$ observed that exosomes derived from malignant metastatic and non-metastatic cell lines displayed reduced stiffness and adhesion compared to exosomes derived from non-malignant cell lines. Furthermore, $\mathrm{EV}$ membrane rigidity has seen to be microenvironmental $\mathrm{pH}$-dependent ${ }^{68}$ and low $\mathrm{pH}$ levels might provide more fluidity of EV membranes promoting fusion and uptake in target cells ${ }^{69}$.

*** Insert Table 2.1***

Table 2.1 EV physicochemical properties. Reported data have been obtained from Thery et al. ${ }^{39}$ for EV size, from Lässer et al. ${ }^{55}$ for EV density, from Jamaly et al. ${ }^{60}$ for EV particle content, from Calò et al. ${ }^{66}$ for EV Young's modulus measurement, from Soares Martins et al. ${ }^{63}$ for EV $\zeta$-potential data and from Vorselen et al. ${ }^{56}$ for EV stiffness. Notice that the reported $\zeta$-potential refers to serum EVs. $\zeta$ potential varies significantly depending on EV media (e.g. saliva,urine, blood, cerebrospinal fluid), and oscillates between $-15 \mathrm{mV}$ and $-34 \mathrm{mV}$. Young's modulus is referred to $\mathrm{EV}$ derived from Saccharomyces cerevisiae. Multilamellar EV stiffness is referred to vesicles with 5 lipid bilayers. Unknown parameters are marked as "n.r." (not reported) and $\rho=$ density.

\subsection{EV separation and characterization}

EV separation methods. EVs can be isolated from biological sources, e.g. biofluids or culture media ${ }^{70}$, by ultrafiltration, precipitation, consecutive centrifugation and ultracentrifugation ${ }^{71}$, size exclusion chromatography $(\mathrm{SEC})^{72}$, density gradient ${ }^{73}$, immunopurification ${ }^{72}$, Tangential Flow Filtration $(T F F)^{74}$ and other microfluidic devices ${ }^{73}$. All separation methods influence EV formulations, to different extents. The correct approach should be adopted after careful evaluation of yield, volume of biofluid needed to be processed and EV source. Furthermore, EV final application is crucial in the selection of separation protocols. Although guidelines to trace and propose a common practice have been recently shared by the scientific community, a standardized practice in EV separation is currently lacking ${ }^{39,75,76}$. Standardization is mandatory in the clinical setting, where the handling and processing of EV preparations must be highly reproducible and robust. Moreover, the 
translation of EVs as drug delivery systems requires cost-effective isolation protocols scalable to massive volumes of samples. For example, ultracentrifugation (over 100,000 $\mathrm{g}$ ) and precipitation by "salting out" with polyethylene glycol (PEG) do not allow large-scale EV purification or pure preparations, which should be obtained by SEC and TFF ${ }^{74,75}$. Nevertheless, both TFF and SEC do not provide the separation between EVs and other biogenic nanoparticles (e.g. lipoproteins) present in biological fluids, and they should be combined with more specific methods ${ }^{77}$. In immunopurification, microbeads are conjugated with specific antibodies against EV markers and vesicles can be purified via antibody-EV protein affinity. As for ultracentrifugation and precipitation, immunopurification is difficult to use in mass-scale production ${ }^{77}$.

EV characterization. Currently, most EV formulations are characterized according to the Minimal Information for Studies of EVs (MISEV 2014) ${ }^{78}$ and the updated MISEV $2018^{39}$. Optical and nonoptical methods have been applied to define EV size distribution ${ }^{58}$. Conventional optical approaches comprise Dynamic Light Scattering (DLS), measuring the fluctuations of the light scattering due to particle Brownian motion ${ }^{58}$, combined or not with Fluorescence Correlation Spectroscopy (FCS) ${ }^{79}$, Nanoparticle Tracking Analysis (NTA), tracking the motion of EVs and calculating the diameter by the Stokes-Einstein equation ${ }^{73}$, Surface Plasmon Resonance devices, which detect ligand binding by calculating the spectral shift of the surface ${ }^{73}$ and Electron ${ }^{73}$ and Atomic Force Microscopy ${ }^{58}$, which can provide also information on structure and "wellness-state" of single EVs. On the other hand, non-optical techniques include impedance-based methods, such as Resistive Pulse Sensing (RPS), in which EV physical properties are reflected into the variation on the current or voltage of the sensor $^{80}$. NTA can be also employed for vesicle quantification, together with nano flow cytometry ${ }^{81}$ and colorimetric nanoplasmonic assay ${ }^{82}$, which can be also applied to check EV sample purity. Instead, biochemical EV characterization can be performed by western blotting, mass spectrometry analysis ${ }^{73}$, enzyme-linked immunosorbent assays ${ }^{75}$ or recently by nano flow cytometry ${ }^{81}$ and nano-FACS (nano-scale Fluorescence-Activated Cell Sorting) ${ }^{83}$.

\subsection{Medical translation of EVs}

EVs present outstanding medical translational opportunities as outlined by the following examples, with specific focus on EV application as therapeutics (drug delivery systems, vaccines) and in diagnostics (biomarkers). Medical applications of EVs in regenerative medicine will be further discussed in section 6 . 
Drug delivery. Nanosized vectors with superior targeting and delivery performance will certainly improve therapy and disease outcome. EVs provide better targeting both in vitro and in vivo, are biocompatible and benefit of increased stability compared to synthetic nanoparticles ${ }^{84}$. Furthermore, due to their heterogeneous structure, EVs can be loaded with both hydrophilic and hydrophobic moieties.

EVs perfectly fulfill the role of carrier for diverse therapeutics, such as RNAs ${ }^{85}$, chemotherapeutics ${ }^{86}$ and small molecules ${ }^{87}$ outperforming synthetic vectors under many aspects (e.g. targeting, toxicity, clearance and stability ${ }^{88}$ ). An example is given by Qu et al. ${ }^{89}$, who have loaded mouse blood EVs with dopamine to treat mouse model of Parkinson's disease. The authors showed that exosomes delivered efficiently dopamine across the Blood-Brain Barrier (BBB) based on the transferrin-transferrin receptor (TfR) interaction, improving disease condition in vivo.

Vaccination. EVs are potent modulators of the immune system ${ }^{90}$. Depending on their origin, cargo, and surface molecules, EVs can both improve or suppress immune response. Due to their immunomodulatory properties EVs, in particular the ones derived from prokaryotic cells, have been explored as vaccines or immunostimulatory agents. For instance, EVs produced by parasites carry a significant number of antigens and can be exploited as vaccine to contrast helminth infections ${ }^{91}$. Moreover, it is known that tumors elicit immune escape through the release of soluble factors and EVs carrying immunosuppressive molecules ${ }^{92}$. However, tumor EVs also represent an interesting source of tumor antigens and could possibly be used as anticancer vaccines in the next years. For instance, with tailored modifications, Morishita et al. ${ }^{93}$ created a delivery system based on tumor EVs, able to induce dendritic cells activation and improve in vivo anti-tumoral response in model mice.

Diagnostics. EVs are enumerated among the most promising, easily accessible source of biomarkers. As a direct result, fluid biopsy - which was primarily devised as a non-invasive method to track circulating tumor cells (e.g. for relapse monitoring), is starting to get complemented with (or replaced by) the analysis of circulating tumor EVs, which transport very valuable information for diagnostics ${ }^{94}$. EVs readily supply two different sources of markers: molecular and biophysical. Molecular information is represented by EV structural components and cargo: proteins, carbohydrates and nucleic acids, all shielded within a lipid bilayer. To date, specific "molecular fingerprints" made of proteins and RNAs have been recognized as marker for (cancer and non-cancer) diseases affecting liver ${ }^{51}$, kidney ${ }^{95}$ and many other organs ${ }^{96,97}$, together with systemic and neurological diseases ${ }^{98}$. Biophysical information is denoted by EV colloidal properties, such as EV size, concentration and mechanical properties. For instance, altered levels of EVs are reported in pancreatic cancer ${ }^{99}$, multiple myeloma ${ }^{59}$ and others ${ }^{100,}$ 
${ }^{101}$, while EV size showed to be significantly different between prostate cancer patients and healthy controls $^{102}$. As mentioned above, EV mechanical properties are likely to be very important for EV interactions with cell membrane and uptake kinetics. For example, altered stiffness and adhesion properties of malignant cell line-derived EVs might have a role in loss of endothelial integrity and complement activation, facilitating the transendothelial EV passage and inducing tumor growth and metastatic lesion formation ${ }^{67}$.

\section{Regenerative properties of EVs}

\subsection{Why EVs?}

As stated earlier in this chapter, recent investigation suggests that the therapeutic efficacy of SCs essentially depends on their secretome ${ }^{57}$, consisting in a mixture of well-known soluble factors (described in Section 4) and the newly discovered EVs, which synergistically cooperate in the regenerative process. Indeed, only in the last few years SC-derived EVs (SC-EVs) not only have been conceived and integrated as part of SC secretome but they also emerged for their biological properties able to mirror the ones of the secreting cells ${ }^{103,104}$. It is now clear that the main therapeutic effect of the secretome may be largely attributed to the constituent EVs within ${ }^{105}$. EV function is related to the role of their bio-active content (proteins, lipids, genetic information) transported to target cells. Their involvement in physiological (i.e. stem cell maintenance, tissue repair, immune surveillance) and pathological events (i.e. carcinogenesis, tumor progression, proinflammatory phenotype) has been indicated by several studies ${ }^{106-108}$. SC-EVs exert immuno-modulatory, as well as regenerative influences, and efficiently mimic the therapeutic effects of SCs alone. Moreover, cell-free delivery of bioactive cargos by EVs induces the same beneficial responses as SC transplantation. Several studies proved that MSC-derived conditioned media (MSC-CM) preserve many therapeutic properties of progenitor cells, and EVs secreted by MSCs upon transplantation might concur to the healing processes ${ }^{109}$. EVs offer remarkable benefits over conventional cell-therapy, since they do not have a nucleus, cannot undergo to neoplastic transformation, are stable to freezing/thawing cycles and can be loaded with many small therapeutic molecules. They possess excellent biocompatibility and biostability characteristics. Being nanosized particles allows them to avoid the pulmonary first-pass effect and to penetrate deep inside tissues ${ }^{110}$. Thus, EVs could be exploited in regenerative medicine, promoting repair and regeneration of damaged target tissues ${ }^{111,112}$. 


\subsection{Preclinical studies}

EVs are currently applied as therapeutics for regenerative medicine in different preclinical studies. Particularly, EVs derived from SCs and immune cells, namely macrophages and dendritic cells, are so far the most studied for regenerative and immunomodulatory applications, although other cell sources have been explored.

MSCs secrete high number of EVs (MSC-EVs) which are highly exploited due to their inability to induce tumors or trigger the host immune system ${ }^{113}$.

Natural or engineered SC-EVs have regenerative effects and unique features that have been exploited in the design of tissue engineering approaches ${ }^{114,115}$. Furthermore, EV regenerative properties have been studied in several in vitro and in vivo models of tissue injury (summarized in Table 2.2), such as lung ${ }^{116}$, liver ${ }^{117}$, and colon injury ${ }^{118}$, as well as myocardial infarction, hereditary or traumatic skin conditions ${ }^{119,}{ }^{120}$, cerebral artery occlusion ${ }^{40}$ and kidney fibrosis ${ }^{121}$. For example, Kholia et al. ${ }^{121}$ investigated the role of human liver SC-EVS (HLSC-EVs) in tubular regeneration and interstitial fibrosis in chronic kidney disease (CKD) mouse model. They demonstrated that HLSC-EVs might act as therapeutic agents in CKD by downregulating pro-fibrotic genes such as alpha smooth muscle actin, Collagen $1 \mathrm{a} 1$ and TGF 1 , showing that the therapeutic effects of MSC-derived EVs mirror those of MSCs. Recently, Zhang et al. ${ }^{122}$ reported that transplantation of both small intestinal submucosa-extracellular matrix seeded with gingival MSCs (GMSCs) and GMSC-EVs promotes the recovery of tongue epithelium papillae, taste bud regeneration and re-innervation in rat model. In 2018, Mohammed et al. ${ }^{123}$ described a possible application of ADSCs and ADSC-EVs in periodontal regeneration. Several authors reported that MSC-EVs can promote bone regeneration ${ }^{124-126}$, angiogenesis in the newly formed tissue ${ }^{127}$, and cartilage repair ${ }^{128}$. Furthermore, recent works have shown that natural SC-EV-based treatments can ameliorate Diabetic Erectile Dysfunction $(D E D)^{129}$, reduce microglia-mediated neuroinflammation ${ }^{130}$, and promote skin wound healing ${ }^{131}$ and nerve sciatic restoration ${ }^{132}$ in rat models. For instance, Ma and colleagues ${ }^{132}$ observed that human Umbilical Cord-MSC (hUCMSC)-EVs promoted axon regeneration and restoration of motor function in rat models of sciatic nerve transection. They also demonstrated that hUCMSC-EVs modulate the inflammation in the damaged nerve, downregulating inflammatory interleukins (IL)- 6 and IL-1 $\beta$, and increasing anti-inflammatory responses. Effects of SC-EVs in maintenance of self-renewal, differentiation or cell fate determination are mostly modulated by EV-small non-coding RNA (sncRNA), including micro RNA 
(miRNA), small nucleolar RNA (snoRNA), RNA transfer (tRNA) or small nuclear RNA (snRNA) ${ }^{133}$. RNA sequencing experiments revealed that EVs preserve characteristic profiles of sncRNA, depending on the stem cells source ${ }^{133}$. According to this study, MSC-derived EVs resulted enriched in sncRNA involved in osteogenesis, chondrogenesis and adipogenesis regulation.

Recently, small non-coding genetic material has been studied as potential molecular therapeutics for the treatment of a broad range of life-threatening pathologies. Small natural or synthetic RNAs regulate the expression of target genes involved in cell cycle, and migration, and in other physiological (angiogenesis) and pathological processes (inflammation). Non-coding RNAs are characterized by low or absent toxicity, and high selectivity toward the target genes ${ }^{134}$. However, if administered, RNA molecules suffer from poor stability and high blood clearance requiring dedicated biocompatible nano-vehicles among which EVs. Hu et al. ${ }^{135}$ reported that astrocyte-EVs loaded with siRNAs targeting proinflammatory IncRNA-Cox2 and administered intranasally restored microglia phagocytic activity in mice treated with morphine.

Furthermore, miRNAs have been also explored as therapeutics for cardiovascular diseases. EVs secreted from HEK293T cells and naturally enriched in miRNA-21 were able to protect cardiomyocytes from apoptosis promoting a cardiac function recovery in mouse models till four weeks after miR21-EV treatment ${ }^{120}$.

Benefits of MSC-EVs have been also observed in in vivo models of brain injury. Interestingly, the administration of MSC-EVs promoted neurogenesis processes by the formation of new synapses, and regulated anti-inflammatory responses together with microglial cells ${ }^{136}$. Recently, tweaked and engineered EVs have been used as biocompatible nanocarriers for endochondral repair ${ }^{137}$, cardiac 119 and thymus ${ }^{138}$ tissue regeneration, and retinal diseases ${ }^{139}$. Banfai et al. ${ }^{138}$ showed that EVs derived from transgenic Thymus Epithelial Cells (TEC) overexpressing Wnt4 and Wnt4-pathway activator miR27b (inhibitors of thymic adipose involution) counteract adipose transformation in a cellular aging model. Because of their biocompatibility, EVs could also be applied as therapeutic systems in neurodegenerative disorders. In fact, EVs are known to be able to cross endothelial barriers such as the blood brain barrier (BBB) without inducing immune responses ${ }^{89}$. Finally, EVs released from immune cells (monocytes, granulocytes and lymphocytes) play a pivotal role in modulation of innate and adaptive immune response by mediating transfer of information between the two immunological pathways ${ }^{140}$. Several studies showed that EVs released by immune cells modulate neovascularization and angiogenesis but the specific role of EVs in this process has 
not been clarified. Immune cell derived-EVs exhibit both pro-angiogenic and anti-angiogenic potentials depending on the parental cells, microenvironment conditions and stimuli involved in their production ${ }^{141}$. Neutrophils pre-treated with $\mathrm{N}$-formylmethionyl-leucyl-phenylalanine (fMLP) secrete EVs with anti-inflammatory properties, whereas neutrophils pre-incubated with HUVEC cells before administration of fMLP produce EVs with pro-inflammatory potential ${ }^{141}$.

$* * *$ Insert Table $2.2 * * *$ 
Table 2.2 Recent EV-based preclinical studies. HLSCs, human Liver Stem Cells; TNBS, 2,4,6trimitrobenzen sulfonic acid; HEK, Human Embryonic Kidney; GMSCs, Gingival Mesenchymal Stem Cells; ESC-MSCs, Embryonic Stem Cell-derived Mesenchymal Stem Cells; hUSCs, human Urinederived Stem Cells; TECs, Thymic Epithelial Cells; BALB/C, TEP1 primary-derived; HPDLSCs, Human Periodontal-Ligament Stem Cells.

\subsection{Clinical studies}

To date, few clinical trials in regenerative medicine based on EVs have been led, including patient treatment for Graft-versus-host ${ }^{142}$ and chronic kidney diseases ${ }^{143}$ (GVHD and CKD). Recently, two phase 1 clinical trials have started, focused on studying the effects of BM-MSC-derived EVs in Bronchopulmonary Dysplasia (NCT03857841) and of MSCs enriched in miR-124 in patients with Acute Ischemic Stroke (NCT03384433) (https://clinicaltrials.gov/). In the study conducted by Kordelas and colleagues ${ }^{142}$, a 20-year old therapy-refractory GvHD female patient was treated with EVs derived from BM-MSCs. MSC-conditioned media derived from bone marrow of four donors have been filtered and small EVs have been isolated via PEG-precipitation. All the vesicle formulations were tested in vitro on patient-derived peripheral blood mononucleated (PBM) and natural killer (NK) cells, in order to prevent any unexpected immune response in vivo. During the therapy, MSC-EVs were administrated every 2-3 days along several months. Mucosal and cutaneous GVHD decreased within two weeks and was stable even after four months from the treatment. Finally, numerous companies have emerged in this field, with the aim to develop EV-based therapeutics, as for example, Capricor Therapeutics Inc. which develops cardiac-derived stem cells and their EVs to repair damaged heart tissue ${ }^{110}$.

\subsection{Limits of EV applications in clinical treatments}

Despite some preclinical promising data, EV-based therapeutic approaches are hindered by several issues $^{76}$. First, the lack of methods allowing isolation of pure EV populations as well as standardized characterization procedures remain critical points that unavoidably limit clinical setting. Second, vesicle biodistribution and circulation kinetics have not yet been defined. Non-invasive imaging techniques and the use of animal models could give concrete answers establishing EV mode of action and optimal doses. Moreover, the elucidation of the mechanism of the significant accumulation of EVs in same organs (e.g. lungs or liver) is required for their practical application. Development of both organ-on-a-chip models and engineered nanoformulations are contributing to improve drug transport providing higher targeting properties. Localized EV releasing might be 
assured by tissue engineering systems, including hydrogels, nanotubes or polymeric biomaterials. For instance, Yerneni and colleagues ${ }^{144}$ developed a bioprinting exosome-like extracellular vesicles microenvironment using macrophage-derived exosomes bioprinted on collagen type-I substrate. Furthermore, this bioprinting technology can be directly translated to in vivo applications for localized exosome delivery to tissues. Finally, analysis of modified EVs is useful to control their pharmacokinetics, but it is not possible to exclude side effects of tailoring on EV biodistribution and target-tissue delivery.

\section{Conclusions}

Extracellular vesicles have gained much importance in the last few years since they are emerging as highly potent therapeutic bio nanoparticles in regenerative medicine, due to their capacity to recapitulate the beneficial properties of originating cells without proliferation issues. The field is still at the beginning and will require significant contributions to understand the complexity of EV biology, distribution and uptake mechanisms. Furthermore, together with basic knowledge, in order to advance the science and later-stage clinical applications, some issues must be faced to shed light on the real potentiality of EVs as therapeutic agents. Among the most important, the wide variability in EV preparations, including EV cell source and culture conditions, EV separation methods applied and the development of standardized quality and functional assays. A practical approach has recently been proposed for a particular set of MSC derived EVs, namely MSC-small EVs (MSC-sEVs), by Society for Clinical Research and Translation of Extracellular Vesicles Singapore (SOCRATES), International Society for Cell and Gene Therapy (ISCT), International Society for

Extracellular Vesicles (ISEV), and International Society of Blood Transfusion (ISBT) societies. The goal of this approach is "to develop a set of minimal quantifiable metrics to harmonize the definition of MSC-sEVs and provide a denominator for comparative manufacturing and functional testing of different preparations"75. This will lead to define physical and biological characteristics of MSCsEVs and develop assays for their measurement. After defining these important structural characteristics of EVs, it will be necessary to establish appropriate functional assays to examine EV therapeutic efficiency, connecting the biological and biophysical information to EV activity. Finally the bottleneck for EV clinical translation remains the scale-up production, although this could be possibly addressed by the use of bioreactors followed by TFF operations ${ }^{110}$. 
In conclusion, EVs either natural or modified with therapeutic agents are envisioned to find increasing applications in the field of regenerative medicine, and together with the development of other technologies will synergistically expand the portfolio of regenerative strategies presently in use.

\section{Acknowledgements}

This work was supported by MIUR through PRIN 2017E3A2NR_004 project to A.R. and P.B., Center for Colloid and Surface Science (CSGI) through the evFOUNDRY project, Horizon 2020- Future and emerging technologies (H2020-FETOPEN), ID: 801367 to P.B, A.R. and L.P. and Mayo Clinic in Florida Focused Research Team Program to S.B. 


\section{References}

1. Tazaki, A.; Tanaka, E. M.; Fei, J. F., Salamander spinal cord regeneration: The ultimate positive control in vertebrate spinal cord regeneration. Dev Biol 2017, 432 (1), 63-71.

2. Mason, C.; Dunnill, P., A brief definition of regenerative medicine. Regenerative Medicine 2007, 3 (1), 1-5.

3. Vacanti, C. A., The history of tissue engineering. J Cell Mol Med 2006, 10 (3), 56976.

4. Colombo, M.; Raposo, G.; Théry, C., Biogenesis, Secretion, and Intercellular Interactions of Exosomes and Other Extracellular Vesicles. Annual Review of Cell and Developmental Biology 2014, 30 (1), 255-289.

5. Weissman, I. L., Stem cells: units of development, units of regeneration, and units in evolution. Cell 2000, 100 (1), 157-68.

6. Ciccocioppo, R.; Cantore, A.; Chaimov, D.; Orlando, G., Regenerative medicine: the red planet for clinicians. Intern Emerg Med 2019.

7. Ayala-Cuellar, A. P.; Kang, J.-H.; Jeung, E.-B.; Choi, K.-C., Roles of Mesenchymal Stem Cells in Tissue Regeneration and Immunomodulation. Biomol Ther (Seoul) 2019, 27 (1), 25 33.

8. Orlando, G.; Murphy, S. V.; Bussolati, B.; Clancy, M.; Cravedi, P.; Migliaccio, G.; Murray, P., Rethinking Regenerative Medicine From a Transplant Perspective (and Vice Versa). Transplantation 2019, 103 (2), 237-249.

9. Cossu, G.; Birchall, M.; Brown, T.; De Coppi, P.; Culme-Seymour, E.; Gibbon, S.; Hitchcock, J.; Mason, C.; Montgomery, J.; Morris, S.; Muntoni, F.; Napier, D.; Owji, N.; Prasad, A.; Round, J.; Saprai, P.; Stilgoe, J.; Thrasher, A.; Wilson, J., Lancet Commission: Stem cells and regenerative medicine. The Lancet 2018, 391 (10123), 883-910.

10. Baldari, S.; Di Rocco, G.; Piccoli, M.; Pozzobon, M.; Muraca, M.; Toietta, G., Challenges and Strategies for Improving the Regenerative Effects of Mesenchymal Stromal CellBased Therapies. Int J Mol Sci 2017, 18 (10).

11. Bason, C.; Gallorini, M.; Berardi, A., The Extracellular Matrix, Growth Factors and Morphogens in Biomaterial Design and Tissue Engineering 2018, 3-26.

12. Bason, C.; Gallorini, M.; Berardi, A., The Extracellular Matrix, Growth Factors and Morphogens in Biomaterial Design and Tissue Engineering. 2018; pp 3-26.

13. Dzobo, K.; Thomford, N. E.; Senthebane, D. A.; Shipanga, H.; Rowe, A.; Dandara, C.; Pillay, M.; Motaung, K., Advances in Regenerative Medicine and Tissue Engineering: Innovation and Transformation of Medicine. Stem Cells Int 2018, 2018, 2495848. 
14. Moroni, L.; Burdick, J. A.; Highley, C.; Lee, S. J.; Morimoto, Y.; Takeuchi, S.; Yoo, J. J., Biofabrication strategies for 3D in vitro models and regenerative medicine. Nat Rev Mater 2018, 3 (5), 21-37.

15. Li, W.; Wu, D.; Zhu, S.; Liu, Z.; Luo, B.; Lu, L.; Zhou, C., Sustained release of plasmid DNA from PLLA/POSS nanofibers for angiogenic therapy. 2019; Vol. 365.

16. Yang, L.; Chueng, S.-T. D.; Li, Y.; Patel, M.; Rathnam, C.; Dey, G.; Wang, L.; Cai, L.; Lee, K.-B., A biodegradable hybrid inorganic nanoscaffold for advanced stem cell therapy. Nature Communications 2018, 9 (1), 3147.

17. Abu-Khader, A.; Law, K. W.; Jahan, S.; Manesia, J. K.; Pasha, R.; Hovey, O.; Pineault, N., Paracrine Factors Released by Osteoblasts Provide Strong Platelet Engraftment Properties. Stem Cells 2019, 37 (3), 345-356.

18. Giampà, C.; Alvino, A.; Magatti, M.; Silini, A. R.; Cardinale, A.; Paldino, E.; Fusco, F. R.; Parolini, O., Conditioned medium from amniotic cells protects striatal degeneration and ameliorates motor deficits in the R6/2 mouse model of Huntington's disease. Journal of cellular and molecular medicine 2019, 23 (2), 1581-1592.

19. Mitchell, R.; Mellows, B.; Sheard, J.; Antonioli, M.; Kretz, O.; Chambers, D.; Zeuner, M.-T.; Tomkins, J. E.; Denecke, B.; Musante, L.; Joch, B.; Debacq-Chainiaux, F.; Holthofer, H.; Ray, S.; Huber, T. B.; Dengjel, J.; De Coppi, P.; Widera, D.; Patel, K., Secretome of adipose-derived mesenchymal stem cells promotes skeletal muscle regeneration through synergistic action of extracellular vesicle cargo and soluble proteins. Stem Cell Research \& Therapy 2019, $10(1), 116$.

20. Ferreira, J. R.; Teixeira, G. Q.; Santos, S. G.; Barbosa, M. A.; Almeida-Porada, G.; Gonçalves, R. M., Mesenchymal Stromal Cell Secretome: Influencing Therapeutic Potential by Cellular Pre-conditioning. Frontiers in immunology 2018, 9, 2837-2837.

21. Galun, E.; Rose-John, S., The regenerative activity of interleukin-6. Methods Mol Biol 2013, 982, 59-77.

22. Sane, M. S.; Misra, N.; Mousa, O. M.; Czop, S.; Tang, H.; Khoo, L. T.; Jones, C. D.; Mustafi, S. B., Cytokines in umbilical cord blood-derived cellular product: a mechanistic insight into bone repair. Regen Med 2018, 13 (8), 881-898.

23. Barhanpurkar-Naik, A.; Mhaske, S. T.; Pote, S. T.; Singh, K.; Wani, M. R., Interleukin-3 enhances the migration of human mesenchymal stem cells by regulating expression of CXCR4. Stem Cell Res Ther 2017, 8 (1), 168. 
24. Heo, S. C.; Jeon, E. S.; Lee, I. H.; Kim, H. S.; Kim, M. B.; Kim, J. H., Tumor necrosis factor-alpha-activated human adipose tissue-derived mesenchymal stem cells accelerate cutaneous wound healing through paracrine mechanisms. J Invest Dermatol 2011, 131 (7), 1559-67.

25. Mitchell, A. C.; Briquez, P. S.; Hubbell, J. A.; Cochran, J. R., Engineering growth factors for regenerative medicine applications. Acta biomaterialia 2016, 30, 1-12.

26. Hodgkinson, T.; Shen, B.; Diwan, A.; Hoyland, J.; Richardson, S., Therapeutic potential of growth differentiation factors in the treatment of degenerative disc diseases. 2019.

27. Chen, T. M.; Chen, Y. H.; Sun, H. S.; Tsai, S. J., Fibroblast growth factors: Potential novel targets for regenerative therapy of osteoarthritis. Chin J Physiol 2019, 62 (1), 2-10.

28. Fu, Y.; Karbaat, L.; Wu, L.; Leijten, J.; Both, S. K.; Karperien, M., Trophic Effects of Mesenchymal Stem Cells in Tissue Regeneration. Tissue Eng Part B Rev 2017, 23 (6), 515-528.

29. Shen, H.; Zhou, E.; Wei, X.; Fu, Z.; Niu, C.; Li, Y.; Pan, B.; Mathew, A. V.; Wang, X.; Pennathur, S.; Zheng, L.; Wang, Y., High density lipoprotein promotes proliferation of adipose-derived stem cells via S1P1 receptor and Akt, ERK1/2 signal pathways. Stem Cell Res Ther 2015, 6, 95 .

30. Tsatralis, T.; Ridiandries, A.; Robertson, S.; Vanags, L. Z.; Lam, Y. T.; Tan, J. T.; Ng, M. K.; Bursill, C. A., Reconstituted high-density lipoproteins promote wound repair and blood flow recovery in response to ischemia in aged mice. Lipids Health Dis 2016, 15 (1), 150.

31. Ding, B.-S.; Liu, C. H.; Sun, Y.; Chen, Y.; Swendeman, S. L.; Jung, B.; Chavez, D.; Cao, Z.; Christoffersen, C.; Nielsen, L. B.; Schwab, S. R.; Rafii, S.; Hla, T., HDL activation of endothelial sphingosine-1-phosphate receptor-1 (S1P(1)) promotes regeneration and suppresses fibrosis in the liver. JCI Insight 2016, 1 (21), e87058-e87058.

32. Giannoni, P.; Hunziker, E. B., Release kinetics of transforming growth factor-beta1 from fibrin clots. Biotechnol Bioeng 2003, 83 (1), 121-3.

33. Janeczek, A. A.; Scarpa, E.; Horrocks, M. H.; Tare, R. S.; Rowland, C. A.; Jenner, D.; Newman, T. A.; Oreffo, R. O.; Lee, S. F.; Evans, N. D., PEGylated liposomes associate with Wnt3A protein and expand putative stem cells in human bone marrow populations. Nanomedicine (Lond) 2017, 12 (8), 845-863.

34. Yi, C.; Liu, D.; Fong, C. C.; Zhang, J.; Yang, M., Gold nanoparticles promote osteogenic differentiation of mesenchymal stem cells through p38 MAPK pathway. ACS Nano 2010, 4 (11), 6439-48.

35. Heo, d. n.; Ko, W.-K.; Bae, M. S.; Bok Lee, J.; Lee, D.-W.; Byun, W.; Hoon Lee, C.; Kim, E.; Jung, B.-Y.; Kwon, I. K., Enhanced bone regeneration with a gold nanoparticlehydrogel complex. 2014; Vol. 2. 
36. Trams, E. G.; Lauter, C. J.; Salem, N., Jr.; Heine, U., Exfoliation of membrane ectoenzymes in the form of micro-vesicles. Biochim Biophys Acta 1981, 645 (1), 63-70.

37. Yanez-Mo, M.; Siljander, P. R.; Andreu, Z.; Zavec, A. B.; Borras, F. E.; Buzas, E. I.; Buzas, K.; Casal, E.; Cappello, F.; Carvalho, J.; Colas, E.; Cordeiro-da Silva, A.; Fais, S.; Falcon-Perez, J. M.; Ghobrial, I. M.; Giebel, B.; Gimona, M.; Graner, M.; Gursel, I.; Gursel, M.; Heegaard, N. H.; Hendrix, A.; Kierulf, P.; Kokubun, K.; Kosanovic, M.; Kralj-Iglic, V.; KramerAlbers, E. M.; Laitinen, S.; Lasser, C.; Lener, T.; Ligeti, E.; Line, A.; Lipps, G.; Llorente, A.; Lotvall, J.; Mancek-Keber, M.; Marcilla, A.; Mittelbrunn, M.; Nazarenko, I.; Nolte-'t Hoen, E. N.; Nyman, T. A.; O'Driscoll, L.; Olivan, M.; Oliveira, C.; Pallinger, E.; Del Portillo, H. A.; Reventos, J.; Rigau, M.; Rohde, E.; Sammar, M.; Sanchez-Madrid, F.; Santarem, N.; Schallmoser, K.; Ostenfeld, M. S.; Stoorvogel, W.; Stukelj, R.; Van der Grein, S. G.; Vasconcelos, M. H.; Wauben, M. H.; De Wever, O., Biological properties of extracellular vesicles and their physiological functions. J Extracell Vesicles 2015, 4, 27066.

38. Ratajczak, J.; Miekus, K.; Kucia, M.; Zhang, J.; Reca, R.; Dvorak, P.; Ratajczak, M. Z., Embryonic stem cell-derived microvesicles reprogram hematopoietic progenitors: evidence for horizontal transfer of mRNA and protein delivery. Leukemia 2006, 20 (5), 847-56.

39. Thery, C.; Witwer, K. W.; Aikawa, E.; Alcaraz, M. J.; Anderson, J. D.; et al., Minimal information for studies of extracellular vesicles 2018 (MISEV2018): a position statement of the International Society for Extracellular Vesicles and update of the MISEV2014 guidelines. $J$ Extracell Vesicles 2018, 7 (1), 1535750.

40. Bjorge, I. M.; Kim, S. Y.; Mano, J. F.; Kalionis, B.; Chrzanowski, W., Extracellular vesicles, exosomes and shedding vesicles in regenerative medicine - a new paradigm for tissue repair. Biomater Sci 2017, 6 (1), 60-78.

41. Xu, R.; Rai, A.; Chen, M.; Suwakulsiri, W.; Greening, D. W.; Simpson, R. J., Extracellular vesicles in cancer - implications for future improvements in cancer care. Nat Rev Clin Oncol 2018, 15 (10), 617-638.

42. Andreu, Z.; Yanez-Mo, M., Tetraspanins in extracellular vesicle formation and function. Front Immunol 2014, 5, 442.

43. Cocucci, E.; Meldolesi, J., Ectosomes and exosomes: shedding the confusion between extracellular vesicles. Trends Cell Biol 2015, 25 (6), 364-72.

44. Murphy, D. E.; de Jong, O. G.; Brouwer, M.; Wood, M. J.; Lavieu, G.; Schiffelers, R. M.; Vader, P., Extracellular vesicle-based therapeutics: natural versus engineered targeting and trafficking. Exp Mol Med 2019, 51 (3), 32. 
45. Costa Verdera, H.; Gitz-Francois, J. J.; Schiffelers, R. M.; Vader, P., Cellular uptake of extracellular vesicles is mediated by clathrin-independent endocytosis and macropinocytosis. $J$ Control Release 2017, 266, 100-108.

46. Mulcahy, L. A.; Pink, R. C.; Carter, D. R., Routes and mechanisms of extracellular vesicle uptake. J Extracell Vesicles 2014, 3.

47. Busatto, S.; Giacomini, A.; Montis, C.; Ronca, R.; Bergese, P., Uptake Profiles of Human Serum Exosomes by Murine and Human Tumor Cells through Combined Use of Colloidal Nanoplasmonics and Flow Cytofluorimetric Analysis. Anal Chem 2018, 90 (13), 7855-7861.

48. Skotland, T.; Sandvig, K.; Llorente, A., Lipids in exosomes: Current knowledge and the way forward. Prog Lipid Res 2017, 66, 30-41.

49. Zaborowski, M. P.; Balaj, L.; Breakefield, X. O.; Lai, C. P., Extracellular Vesicles: Composition, Biological Relevance, and Methods of Study. Bioscience 2015, 65 (8), 783-797.

50. Bruno, S.; Grange, C.; Deregibus, M. C.; Calogero, R. A.; Saviozzi, S.; Collino, F.; Morando, L.; Busca, A.; Falda, M.; Bussolati, B.; Tetta, C.; Camussi, G., Mesenchymal stem cellderived microvesicles protect against acute tubular injury. J Am Soc Nephrol 2009, 20 (5), 10531067.

51. Berardocco, M.; Radeghieri, A.; Busatto, S.; Gallorini, M.; Raggi, C.; Gissi, C.; D'Agnano, I.; Bergese, P.; Felsani, A.; Berardi, A. C., RNA-seq reveals distinctive RNA profiles of small extracellular vesicles from different human liver cancer cell lines. Oncotarget 2017, 8 (47), 82920-82939.

52. Spinelli, C.; Adnani, L.; Choi, D.; Rak, J., Extracellular Vesicles as Conduits of NonCoding RNA Emission and Intercellular Transfer in Brain Tumors. Noncoding RNA 2018, 5 (1), 1.

53. Fernando, M. R.; Jiang, C.; Krzyzanowski, G. D.; Ryan, W. L., New evidence that a large proportion of human blood plasma cell-free DNA is localized in exosomes. PloS one 2017, 12 (8), e0183915-e0183915.

54. Jeppesen, D.; Fenix, A.; Franklin, J.; Higginbotham, J.; Zhang, Q.; Zimmerman, L.; Liebler, D.; Ping, J.; Liu, q.; Evans, R.; Fissell, W.; Patton, J.; Rome, L.; Burnette, D.; Coffey, R., Reassessment of Exosome Composition. Cell 2019, 177, 428-445.e18.

55. Lasser, C.; Jang, S. C.; Lotvall, J., Subpopulations of extracellular vesicles and their therapeutic potential. Mol Aspects Med 2018, 60, 1-14.

56. Vorselen, D.; Marchetti, M.; López-Iglesias, C.; Peters, P. J.; Roos, W. H.; Wuite, G. J. L., Multilamellar nanovesicles show distinct mechanical properties depending on their degree of lamellarity. Nanoscale 2018, 10 (11), 5318-5324. 
57. Busatto, S.; Zendrini, A.; Radeghieri, A.; Paolini, L.; Romano, M.; Presta, M.; Bergese, P., The Nanostructured Secretome. Biomaterials Science 2019.

58. Paolini, L.; Zendrini, A.; Radeghieri, A., Biophysical properties of extracellular vesicles in diagnostics. Biomark Med 2018, 12 (4), 383-391.

59. Di Noto, G.; Bugatti, A.; Zendrini, A.; Mazzoldi, E. L.; Montanelli, A.; Caimi, L.; Rusnati, M.; Ricotta, D.; Bergese, P., Merging colloidal nanoplasmonics and surface plasmon resonance spectroscopy for enhanced profiling of multiple myeloma-derived exosomes. Biosens Bioelectron 2016, 77, 518-24.

60. Jamaly, S.; Ramberg, C.; Olsen, R.; Latysheva, N.; Webster, P.; Sovershaev, T.; Brækkan, S. K.; Hansen, J.-B., Impact of preanalytical conditions on plasma concentration and size distribution of extracellular vesicles using Nanoparticle Tracking Analysis. Scientific Reports 2018, $8(1), 17216$

61. Lane, R.; Simon, T.; Vintu, M.; Solkin, B.; Koch, B.; Stewart, N.; Benstead-Hume, G.; Pearl, F. M. G.; Critchley, G.; Stebbing, J.; Giamas, G., Cell-derived extracellular vesicles can be used as a biomarker reservoir for glioblastoma tumor subtyping. Communications Biology 2019, $2(1), 315$.

62. Serrano-Pertierra, E.; Oliveira-Rodríguez, M.; Rivas, M.; Oliva, P.; Villafani, J.; Navarro, A.; Blanco-López, M. C.; Cernuda-Morollón, E., Characterization of Plasma-Derived Extracellular Vesicles Isolated by Different Methods: A Comparison Study. Bioengineering (Basel, Switzerland) 2019, 6 (1), 8.

63. Soares Martins, T.; Catita, J.; Martins Rosa, I.; A. B. da Cruz e Silva, O.; Henriques, A. G., Exosome isolation from distinct biofluids using precipitation and column-based approaches. PLOS ONE 2018, 13 (6), e0198820.

64. Beit-Yannai, E.; Tabak, S.; Stamer, W. D., Physical exosome:exosome interactions. Journal of cellular and molecular medicine 2018, 22 (3), 2001-2006.

65. Vorselen, D.; van Dommelen, S. M.; Sorkin, R.; Piontek, M. C.; Schiller, J.; Döpp, S. T.; Kooijmans, S. A. A.; van Oirschot, B. A.; Versluijs, B. A.; Bierings, M. B.; van Wijk, R.; Schiffelers, R. M.; Wuite, G. J. L.; Roos, W. H., The fluid membrane determines mechanics of erythrocyte extracellular vesicles and is softened in hereditary spherocytosis. Nature Communications 2018, 9 (1), 4960.

66. Calò, A.; Reguera, D.; Oncins, G.; Persuy, M.-A.; Sanz, G.; Lobasso, S.; Corcelli, A.; Pajot-Augy, E.; Gomila, G., Force measurements on natural membrane nanovesicles reveal a composition-independent, high Young's modulus. Nanoscale 2014, 6 (4), 2275-2285. 
67. Whitehead, B.; Wu, L.; Hvam, M. L.; Aslan, H.; Dong, M.; Dyrskjøt, L.; Ostenfeld, M. S.; Moghimi, S. M.; Howard, K. A., Tumour exosomes display differential mechanical and complement activation properties dependent on malignant state: implications in endothelial leakiness. Journal of extracellular vesicles 2015, 4, 29685-29685.

68. Laulagnier, K.; Motta, C.; Hamdi, S.; Roy, S.; Fauvelle, F.; Pageaux, J.-F.; Kobayashi, T.; Salles, J.-P.; Perret, B.; Bonnerot, C.; Record, M., Mast cell- and dendritic cellderived exosomes display a specific lipid composition and an unusual membrane organization. Biochem J 2004, 380 (Pt 1), 161-171.

69. Parolini, I.; Federici, C.; Raggi, C.; Lugini, L.; Palleschi, S.; De Milito, A.; Coscia, C.; Iessi, E.; Logozzi, M.; Molinari, A.; Colone, M.; Tatti, M.; Sargiacomo, M.; Fais, S., Microenvironmental $\mathrm{pH}$ is a key factor for exosome traffic in tumor cells. J Biol Chem 2009, 284 (49), 34211-34222.

70. Paolini, L.; Orizio, F.; Busatto, S.; Radeghieri, A.; Bresciani, R.; Bergese, P.; Monti, E., Exosomes Secreted by HeLa Cells Shuttle on Their Surface the Plasma Membrane-Associated Sialidase NEU3. Biochemistry 2017, 56 (48), 6401-6408.

71. Vescovi, R.; Monti, M.; Moratto, D.; Paolini, L.; Consoli, F.; Benerini, L.; Melocchi, L.; Calza, S.; Chiudinelli, M.; Rossi, G.; Bugatti, M.; Maio, M.; Fonsatti, E.; Farisoglio, C.; Simbolo, M.; Almici, C.; Verardi, R.; Scarpa, A.; Bergese, P.; Manganoni, A.; Facchetti, F.; Vermi, W., Collapse of the Plasmacytoid Dendritic Cell Compartment in Advanced Cutaneous Melanomas by Components of the Tumor Cell Secretome. Cancer Immunol Res 2019, 7 (1), 12-28.

72. Ferreira, A. D. F.; Gomes, D. A., Stem Cell Extracellular Vesicles in Skin Repair. Bioengineering (Basel) 2018, 6 (1).

73. Ingato, D.; Lee, J. U.; Sim, S. J.; Kwon, Y. J., Good things come in small packages: Overcoming challenges to harness extracellular vesicles for therapeutic delivery. $J$ Control Release 2016, 241, 174-185.

74. Busatto, S.; Vilanilam, G.; Ticer, T.; Lin, W. L.; Dickson, D. W.; Shapiro, S.; Bergese, P.; Wolfram, J., Tangential Flow Filtration for Highly Efficient Concentration of Extracellular Vesicles from Large Volumes of Fluid. Cells 2018, 7 (12).

75. Witwer, K. W.; Van Balkom, B. W. M.; Bruno, S.; Choo, A.; Dominici, M.; Gimona, M.; Hill, A. F.; De Kleijn, D.; Koh, M.; Lai, R. C.; Mitsialis, S. A.; Ortiz, L. A.; Rohde, E.; Asada, T.; Toh, W. S.; Weiss, D. J.; Zheng, L.; Giebel, B.; Lim, S. K., Defining mesenchymal stromal cell (MSC)-derived small extracellular vesicles for therapeutic applications. $J$ Extracell Vesicles 2019, 8 (1), 1609206. 
76. Margolis, L.; Sadovsky, Y., The biology of extracellular vesicles: The known unknowns. PLoS Biol 2019, 17 (7), e3000363-e3000363.

77. Somiya, M.; Yoshioka, Y.; Ochiya, T., Drug delivery application of extracellular vesicles; insight into production, drug loading, targeting, and pharmacokinetics. 2017; Vol. 4, p 7392.

78. Lotvall, J.; Hill, A. F.; Hochberg, F.; Buzas, E. I.; Di Vizio, D.; Gardiner, C.; Gho, Y. S.; Kurochkin, I. V.; Mathivanan, S.; Quesenberry, P.; Sahoo, S.; Tahara, H.; Wauben, M. H.; Witwer, K. W.; Thery, C., Minimal experimental requirements for definition of extracellular vesicles and their functions: a position statement from the International Society for Extracellular Vesicles. $J$ Extracell Vesicles 2014, 3, 26913.

79. Montis, C.; Zendrini, A.; Valle, F.; Busatto, S.; Paolini, L.; Radeghieri, A.; Salvatore, A.; Berti, D.; Bergese, P., Size distribution of extracellular vesicles by optical correlation techniques. Colloids Surf B Biointerfaces 2017, 158, 331-338.

80. Ahmad, M. A., Electrical Detection, Identification, and Quantification of Exosomes. IEEE Access 2018, 6, 22817-22826.

81. Friedrich, R.; Block, S.; Alizadehheidari, M.; Heider, S.; Fritzsche, J.; Esbjorner, E. K.; Westerlund, F.; Bally, M., A nano flow cytometer for single lipid vesicle analysis. Lab Chip 2017, 17 (5), 830-841.

82. Maiolo, D.; Paolini, L.; Di Noto, G.; Zendrini, A.; Berti, D.; Bergese, P.; Ricotta, D., Colorimetric nanoplasmonic assay to determine purity and titrate extracellular vesicles. Anal Chem 2015, 87 (8), 4168-76.

83. Morales-Kastresana, A.; Telford, B.; Musich, T. A.; McKinnon, K.; Clayborne, C.; Braig, Z.; Rosner, A.; Demberg, T.; Watson, D. C.; Karpova, T. S.; Freeman, G. J.; DeKruyff, R. H.; Pavlakis, G. N.; Terabe, M.; Robert-Guroff, M.; Berzofsky, J. A.; Jones, J. C., Labeling Extracellular Vesicles for Nanoscale Flow Cytometry. Sci Rep 2017, 7 (1), 1878.

84. Armstrong, J. P. K.; Stevens, M. M., Strategic design of extracellular vesicle drug delivery systems. Adv Drug Deliv Rev 2018, 130, 12-16.

85. Usman, W. M.; Pham, T. C.; Kwok, Y. Y.; Vu, L. T.; Ma, V.; Peng, B.; Chan, Y. S.; Wei, L.; Chin, S. M.; Azad, A.; He, A. B.; Leung, A. Y. H.; Yang, M.; Shyh-Chang, N.; Cho, W. C.; Shi, J.; Le, M. T. N., Efficient RNA drug delivery using red blood cell extracellular vesicles. Nat Commun 2018, 9 (1), 2359.

86. Chulpanova, D. S.; Kitaeva, K. V.; James, V.; Rizvanov, A. A.; Solovyeva, V. V., Therapeutic Prospects of Extracellular Vesicles in Cancer Treatment. Front Immunol 2018, 9, 1534. 
87. Haney, M. J.; Klyachko, N. L.; Zhao, Y.; Gupta, R.; Plotnikova, E. G.; He, Z.; Patel, T.; Piroyan, A.; Sokolsky, M.; Kabanov, A. V.; Batrakova, E. V., Exosomes as drug delivery vehicles for Parkinson's disease therapy. J Control Release 2015, 207, 18-30.

88. Dai, Q.; Wilhelm, S.; Ding, D.; Syed, A. M.; Sindhwani, S.; Zhang, Y.; Chen, Y. Y.; MacMillan, P.; Chan, W. C. W., Quantifying the Ligand-Coated Nanoparticle Delivery to Cancer Cells in Solid Tumors. ACS Nano 2018, 12 (8), 8423-8435.

89. Qu, M.; Lin, Q.; Huang, L.; Fu, Y.; Wang, L.; He, S.; Fu, Y.; Yang, S.; Zhang, Z.; Zhang, L.; Sun, X., Dopamine-loaded blood exosomes targeted to brain for better treatment of Parkinson's disease. J Control Release 2018, 287, 156-166.

90. Tan, K.; Li, R.; Huang, X.; Liu, Q., Outer Membrane Vesicles: Current Status and Future Direction of These Novel Vaccine Adjuvants. 2018, 9 (783).

91. Mekonnen, G. G.; Pearson, M.; Loukas, A.; Sotillo, J., Extracellular vesicles from parasitic helminths and their potential utility as vaccines. Expert Rev Vaccines 2018, 17 (3), 197-205.

92. Alfonsi, R.; Grassi, L.; Signore, M.; Bonci, D., The Double Face of Exosome-Carried MicroRNAs in Cancer Immunomodulation. Int J Mol Sci 2018, 19 (4).

93. Morishita, M.; Takahashi, Y.; Matsumoto, A.; Nishikawa, M.; Takakura, Y., Exosome-based tumor antigens-adjuvant co-delivery utilizing genetically engineered tumor cellderived exosomes with immunostimulatory CpG DNA. Biomaterials 2016, 111, 55-65.

94. Garcia-Romero, N.; Esteban-Rubio, S.; Rackov, G.; Carrion-Navarro, J.; BeldaIniesta, C.; Ayuso-Sacido, A., Extracellular vesicles compartment in liquid biopsies: Clinical application. Mol Aspects Med 2018, 60, 27-37.

95. Zhang, W.; Zhou, X.; Zhang, H.; Yao, Q.; Liu, Y.; Dong, Z., Extracellular vesicles in diagnosis and therapy of kidney diseases. Am J Physiol Renal Physiol 2016, 311 (5), F844-F851.

96. Fredsoe, J.; Rasmussen, A. K. I.; Mouritzen, P.; Borre, M.; Orntoft, T.; Sorensen, K. D., A five-microRNA model ( $\mathrm{pCaP}$ ) for predicting prostate cancer aggressiveness using cell-free urine. Int J Cancer 2019.

97. Castillo, J.; Bernard, V.; San Lucas, F. A.; Allenson, K.; Capello, M.; Kim, D. U.; Gascoyne, P.; Mulu, F. C.; Stephens, B. M.; Huang, J.; Wang, H.; Momin, A. A.; Jacamo, R. O.; Katz, M.; Wolff, R.; Javle, M.; Varadhachary, G.; Wistuba, II; Hanash, S.; Maitra, A.; Alvarez, H., Surfaceome profiling enables isolation of cancer-specific exosomal cargo in liquid biopsies from pancreatic cancer patients. Ann Oncol 2018, 29 (1), 223-229.

98. Salvi, A.; Vezzoli, M.; Busatto, S.; Paolini, L.; Faranda, T.; Abeni, E.; Caracausi, M.; Antonaros, F.; Piovesan, A.; Locatelli, C.; Cocchi, G.; Alvisi, G.; De Petro, G.; Ricotta, D.; 
Bergese, P.; Radeghieri, A., Analysis of a nanoparticle-enriched fraction of plasma reveals miRNA candidates for Down syndrome pathogenesis. Int J Mol Med 2019, 43 (6), 2303-2318.

99. Liang, K.; Liu, F.; Fan, J.; Sun, D.; Liu, C.; Lyon, C. J.; Bernard, D. W.; Li, Y.; Yokoi, K.; Katz, M. H.; Koay, E. J.; Zhao, Z.; Hu, Y., Nanoplasmonic Quantification of Tumorderived Extracellular Vesicles in Plasma Microsamples for Diagnosis and Treatment Monitoring. Nat Biomed Eng 2017, 1.

100. Liang, L. G.; Kong, M. Q.; Zhou, S.; Sheng, Y. F.; Wang, P.; Yu, T.; Inci, F.; Kuo, W. P.; Li, L. J.; Demirci, U.; Wang, S., An integrated double-filtration microfluidic device for isolation, enrichment and quantification of urinary extracellular vesicles for detection of bladder cancer. Sci Rep 2017, 7, 46224.

101. Liu, C.; Xu, X.; Li, B.; Situ, B.; Pan, W.; Hu, Y.; An, T.; Yao, S.; Zheng, L., Single-Exosome-Counting Immunoassays for Cancer Diagnostics. Nano Lett 2018, 18 (7), 42264232.

102. Yang, J. S.; Lee, J. C.; Byeon, S. K.; Rha, K. H.; Moon, M. H., Size Dependent Lipidomic Analysis of Urinary Exosomes from Patients with Prostate Cancer by Flow Field-Flow Fractionation and Nanoflow Liquid Chromatography-Tandem Mass Spectrometry. Anal Chem 2017, 89 (4), 2488-2496.

103. Timmers, L.; Lim, S. K.; Arslan, F.; Armstrong, J. S.; Hoefer, I. E.; Doevendans, P. A.; Piek, J. J.; El Oakley, R. M.; Choo, A.; Lee, C. N.; Pasterkamp, G.; de Kleijn, D. P., Reduction of myocardial infarct size by human mesenchymal stem cell conditioned medium. Stem Cell Res 2007, 1 (2), 129-37.

104. van Koppen, A.; Joles, J. A.; van Balkom, B. W.; Lim, S. K.; de Kleijn, D.; Giles, R. H.; Verhaar, M. C., Human embryonic mesenchymal stem cell-derived conditioned medium rescues kidney function in rats with established chronic kidney disease. PLoS One 2012, 7 (6), e38746.

105. Lai, R. C.; Arslan, F.; Lee, M. M.; Sze, N. S.; Choo, A.; Chen, T. S.; Salto-Tellez, M.; Timmers, L.; Lee, C. N.; El Oakley, R. M.; Pasterkamp, G.; de Kleijn, D. P.; Lim, S. K., Exosome secreted by MSC reduces myocardial ischemia/reperfusion injury. Stem Cell Res 2010, 4 (3), 214-22.

106. Quesenberry, P. J.; Aliotta, J.; Deregibus, M. C.; Camussi, G., Role of extracellular RNA-carrying vesicles in cell differentiation and reprogramming. Stem Cell Res Ther 2015, 6, 153.

107. Yuana, Y.; Sturk, A.; Nieuwland, R., Extracellular vesicles in physiological and pathological conditions. Blood Rev 2013, 27 (1), 31-9. 
108. Hosseinkhani, B.; Kuypers, S.; van den Akker, N. M. S.; Molin, D. G. M.; Michiels, L., Extracellular Vesicles Work as a Functional Inflammatory Mediator Between Vascular Endothelial Cells and Immune Cells. Front Immunol 2018, 9, 1789.

109. Gunawardena, T. N. A.; Rahman, M. T.; Abdullah, B. J. J.; Abu Kasim, N. H., Conditioned media derived from mesenchymal stem cell cultures: The next generation for regenerative medicine. J Tissue Eng Regen Med 2019, 13 (4), 569-586.

110. Riazifar, M.; Pone, E. J.; Lotvall, J.; Zhao, W., Stem Cell Extracellular Vesicles: Extended Messages of Regeneration. Annu Rev Pharmacol Toxicol 2017, 57, 125-154.

111. Cossu, G.; Birchall, M.; Brown, T.; De Coppi, P.; Culme-Seymour, E.; Gibbon, S.; Hitchcock, J.; Mason, C.; Montgomery, J.; Morris, S.; Muntoni, F.; Napier, D.; Owji, N.; Prasad, A.; Round, J.; Saprai, P.; Stilgoe, J.; Thrasher, A.; Wilson, J., The Lancet Commissions Lancet Commission: Stem cells and regenerative medicine Executive summary. 2018.

112. Radeghieri, A.; Savio, G.; Zendrini, A.; Di Noto, G.; Salvi, A.; Bergese, P.; Piovani, G., Cultured human amniocytes express hTERT, which is distributed between nucleus and cytoplasm and is secreted in extracellular vesicles. Biochem Biophys Res Commun 2017, 483 (1), 706-711.

113. Cheng, L.; Zhang, K.; Wu, S.; Cui, M.; Xu, T., Focus on Mesenchymal Stem CellDerived Exosomes: Opportunities and Challenges in Cell-Free Therapy. Stem Cells Int 2017, 2017, 6305295.

114. Lamichhane, T. N.; Sokic, S.; Schardt, J. S.; Raiker, R. S.; Lin, J. W.; Jay, S. M., Emerging roles for extracellular vesicles in tissue engineering and regenerative medicine. Tissue Eng Part B Rev 2015, 21 (1), 45-54.

115. Wei, F.; Li, M.; Crawford, R.; Zhou, Y.; Xiao, Y., Exosome-integrated titanium oxide nanotubes for targeted bone regeneration. Acta Biomater 2019, 86, 480-492.

116. Willis, G. R.; Fernandez-Gonzalez, A.; Anastas, J.; Vitali, S. H.; Liu, X.; Ericsson, M.; Kwong, A.; Mitsialis, S. A.; Kourembanas, S., Mesenchymal Stromal Cell Exosomes Ameliorate Experimental Bronchopulmonary Dysplasia and Restore Lung Function through Macrophage Immunomodulation. Am J Respir Crit Care Med 2018, 197 (1), 104-116.

117. Chen, L.; Chen, R.; Kemper, S.; Cong, M.; You, H.; Brigstock, D. R., Therapeutic effects of serum extracellular vesicles in liver fibrosis. Journal of extracellular vesicles 2018, 7 (1), 1461505-1461505.

118. Yang, J.; Liu, X. X.; Fan, H.; Tang, Q.; Shou, Z. X.; Zuo, D. M.; Zou, Z.; Xu, M.; Chen, Q. Y.; Peng, Y.; Deng, S. J.; Liu, Y. J., Extracellular Vesicles Derived from Bone Marrow Mesenchymal Stem Cells Protect against Experimental Colitis via Attenuating Colon Inflammation, Oxidative Stress and Apoptosis. PLoS One 2015, 10 (10), e0140551. 
119. Vandergriff, A.; Huang, K.; Shen, D.; Hu, S.; Hensley, M. T.; Caranasos, T. G.; Qian, L.; Cheng, K., Targeting regenerative exosomes to myocardial infarction using cardiac homing peptide. Theranostics 2018, 8 (7), 1869-1878.

120. Song, Y.; Zhang, C.; Zhang, J.; Jiao, Z.; Dong, N.; Wang, G.; Wang, Z.; Wang, L., Localized injection of miRNA-21-enriched extracellular vesicles effectively restores cardiac function after myocardial infarction. Theranostics 2019, 9 (8), 2346-2360.

121. Kholia, S.; Herrera Sanchez, M. B.; Cedrino, M.; Papadimitriou, E.; Tapparo, M.; Deregibus, M. C.; Brizzi, M. F.; Tetta, C.; Camussi, G., Human Liver Stem Cell-Derived Extracellular Vesicles Prevent Aristolochic Acid-Induced Kidney Fibrosis. Front Immunol 2018, 9 , 1639.

122. Zhang, Y.; Shi, S.; Xu, Q.; Zhang, Q.; Shanti, R. M.; Le, A. D., SIS-ECM Laden with GMSC-Derived Exosomes Promote Taste Bud Regeneration. J Dent Res 2019, 98 (2), 225-233.

123. Mohammed, E.; Khalil, E.; Sabry, D., Effect of Adipose-Derived Stem Cells and Their Exo as Adjunctive Therapy to Nonsurgical Periodontal Treatment: A Histologic and Histomorphometric Study in Rats. Biomolecules 2018, 8 (4).

124. Chu, C.; Wei, S.; Wang, Y.; Wang, Y.; Man, Y.; Qu, Y., Extracellular vesicle and mesenchymal stem cells in bone regeneration: recent progress and perspectives. $J$ Biomed Mater Res A 2019, 107 (1), 243-250.

125. Lu, Z.; Chen, Y.; Dunstan, C.; Roohani-Esfahani, S.; Zreiqat, H., Priming Adipose Stem Cells with Tumor Necrosis Factor-Alpha Preconditioning Potentiates Their Exosome Efficacy for Bone Regeneration. Tissue Eng Part A 2017, 23 (21-22), 1212-1220.

126. Pizzicannella, J.; Gugliandolo, A.; Orsini, T.; Fontana, A.; Ventrella, A.; Mazzon, E.; Bramanti, P.; Diomede, F.; Trubiani, O., Engineered Extracellular Vesicles From Human Periodontal-Ligament Stem Cells Increase VEGF/VEGFR2 Expression During Bone Regeneration. Front Physiol 2019, 10, 512.

127. Zimta, A. A.; Baru, O.; Badea, M.; Buduru, S. D.; Berindan-Neagoe, I., The Role of Angiogenesis and Pro-Angiogenic Exosomes in Regenerative Dentistry. Int J Mol Sci 2019, 20 (2).

128. Zhang, S.; Chuah, S. J.; Lai, R. C.; Hui, J. H. P.; Lim, S. K.; Toh, W. S., MSC exosomes mediate cartilage repair by enhancing proliferation, attenuating apoptosis and modulating immune reactivity. Biomaterials 2018, 156, 16-27.

129. Ouyang, B.; Xie, Y.; Zhang, C.; Deng, C.; Lv, L.; Yao, J.; Zhang, Y.; Liu, G.; Deng, J.; Deng, C., Extracellular Vesicles From Human Urine-Derived Stem Cells Ameliorate Erectile Dysfunction in a Diabetic Rat Model by Delivering Proangiogenic MicroRNA. Sex Med 2019, 7 (2), 241-250. 
130. Thomi, G.; Surbek, D.; Haesler, V.; Joerger-Messerli, M.; Schoeberlein, A., Exosomes derived from umbilical cord mesenchymal stem cells reduce microglia-mediated neuroinflammation in perinatal brain injury. Stem Cell Res Ther 2019, 10 (1), 105.

131. Zhang, B.; Wang, M.; Gong, A.; Zhang, X.; Wu, X.; Zhu, Y.; Shi, H.; Wu, L.; Zhu, W.; Qian, H.; Xu, W., HucMSC-Exosome Mediated-Wnt4 Signaling Is Required for Cutaneous Wound Healing. Stem Cells 2015, 33 (7), 2158-68.

132. Ma, Y.; Dong, L.; Zhou, D.; Li, L.; Zhang, W.; Zhen, Y.; Wang, T.; Su, J.; Chen, D.; Mao, C.; Wang, X., Extracellular vesicles from human umbilical cord mesenchymal stem cells improve nerve regeneration after sciatic nerve transection in rats. J Cell Mol Med 2019, 23 (4), 28222835.

133. Kaur, S.; Abu-Shahba, A. G.; Paananen, R. O.; Hongisto, H.; Hiidenmaa, H.; Skottman, H.; Seppanen-Kaijansinkko, R.; Mannerstrom, B., Small non-coding RNA landscape of extracellular vesicles from human stem cells. Sci Rep 2018, 8 (1), 15503.

134. Pomatto, M. A. C.; Bussolati, B.; D'Antico, S.; Ghiotto, S.; Tetta, C.; Brizzi, M. F.; Camussi, G., Improved Loading of Plasma-Derived Extracellular Vesicles to Encapsulate Antitumor miRNAs. Mol Ther Methods Clin Dev 2019, 13, 133-144.

135. Hu, G.; Liao, K.; Niu, F.; Yang, L.; Dallon, B. W.; Callen, S.; Tian, C.; Shu, J.; Cui, J.; Sun, Z.; Lyubchenko, Y. L.; Ka, M.; Chen, X. M.; Buch, S., Astrocyte EV-Induced lincRNA-Cox2 Regulates Microglial Phagocytosis: Implications for Morphine-Mediated Neurodegeneration. Mol Ther Nucleic Acids 2018, 13, 450-463.

136. Elia, C. A.; Losurdo, M.; Malosio, M. L.; Coco, S., Extracellular Vesicles from Mesenchymal Stem Cells Exert Pleiotropic Effects on Amyloid-beta, Inflammation, and Regeneration: A Spark of Hope for Alzheimer's Disease from Tiny Structures? Bioessays 2019, 41 (4), e1800199.

137. Ferreira, E.; Porter, R. M., Harnessing extracellular vesicles to direct endochondral repair of large bone defects. Bone Joint Res 2018, 7 (4), 263-273.

138. Banfai, K.; Garai, K.; Ernszt, D.; Pongracz, J. E.; Kvell, K., Transgenic Exosomes for Thymus Regeneration. Front Immunol 2019, 10, 862.

139. van der Merwe, Y.; Steketee, M. B., Extracellular Vesicles: Biomarkers, Therapeutics, and Vehicles in the Visual System. Curr Ophthalmol Rep 2017, 5 (4), 276-282.

140. Fendl, B.; Eichhorn, T.; Weiss, R.; Tripisciano, C.; Spittler, A.; Fischer, M. B.; Weber, V., Differential Interaction of Platelet-Derived Extracellular Vesicles With Circulating Immune Cells: Roles of TAM Receptors, CD11b, and Phosphatidylserine. Front Immunol 2018, 9, 2797. 
141. Sanchez-Alonso, S.; Alcaraz-Serna, A.; Sanchez-Madrid, F.; Alfranca, A., Extracellular Vesicle-Mediated Immune Regulation of Tissue Remodeling and Angiogenesis After Myocardial Infarction. Front Immunol 2018, 9, 2799.

142. Kordelas, L.; Rebmann, V.; Ludwig, A. K.; Radtke, S.; Ruesing, J.; Doeppner, T. R.; Epple, M.; Horn, P. A.; Beelen, D. W.; Giebel, B., MSC-derived exosomes: a novel tool to treat therapy-refractory graft-versus-host disease. Leukemia 2014, 28 (4), 970-3.

143. Nassar, W.; El-Ansary, M.; Sabry, D.; Mostafa, M. A.; Fayad, T.; Kotb, E.; Temraz, M.; Saad, A. N.; Essa, W.; Adel, H., Umbilical cord mesenchymal stem cells derived extracellular vesicles can safely ameliorate the progression of chronic kidney diseases. Biomater Res 2016, 20, 21.

144. Yerneni, S.; Whiteside, T.; E. Weiss, L.; Campbell, P., Bioprinting Exosome-Like Extracellular Vesicle Microenvironments. 2019; p e00041. 
NOVEL STRATEGIES TO IMPROVE DELIVERY PERFORMANCES

\title{
Emanuele Mauri
}

Department of Engineering, Università Campus Bio-Medico di Roma, via Alvaro del Portillo 21, 00128 Rome

\section{[NON PRINT ITEMS]}

\begin{abstract}
:
Inducing a desired bioresponse, promoting a targeted therapy or inhibiting potentially adverse reactions are the pivotal challenges in tissue engineering and theranostic fields. Through the smart design of nanomaterials, it is possible to overcome a lot of constrains which significantly reduce the efficiency of the treatments. However, even if the simple combination of different materials could be the first approach to address the biocompatible criteria and some requested physical properties, the use of functionalization strategies can lead the nanomaterial science into an innovative area of selective and versatile nature. Indeed, desired molecules, such as aminoacids, peptides, drugs, paramagnetic compounds, markers, or simple chemical groups can be added improving the mechanical properties, the selectivity towards a specific cell line and the control of the release kinetic of therapeutics. In particular, chemical functionalization methods involve the orthogonal chemistry to form covalent linkages between the nanomaterial and the moieties to be grafted: these bonds can also be stimuli-sensitive (i.e. $\mathrm{pH}$-sensitive, temperature-sensitive, light-sensitive, redox-sensitive) ensuring a tunable effect in the biological environment. On the other hand, physical functionalizations are based on non-covalent interactions and modify the architectural structures of the nanomaterials without affecting their chemical composition. The possibility to design a nanodevice ad-hoc opens new opportunities for the researchers to solve the critical points related to cross the biological barriers and improve the delivery performance.
\end{abstract}

\section{Key Words:}

Chemical functionalization, click chemistry, physical functionalization, polymers, nanoparticles, nanogels, membrane barriers. 


\subsection{Introduction}

The rationale design of nanoscale systems represents the pivotal challenge for a successful strategy aimed to guarantee therapeutic benefits, without side-effects, in the complex scenario of tissue engineering and theranostic. Indeed, nanocarriers are asked to treat constantly evolving diseases through the interactions with biological barriers, the in situ bio-distribution of encapsulated active principles, and the diagnostic targeting. The first generation of nanomaterials was essentially focused on the simple physical or chemical combination of natural and synthetic compounds, exploiting the neat molecular structures and the inner reactive groups. These nanocarriers, such as liposomes, micelles and gold-derived configurations, are also known as the base of nanoparticle formulation[1], and they are characterized by non-specific surface properties, physical- and steric hindrance-driven encapsulation of the payload, and subjected to passive cellular internalization (in particular by the mononuclear phagocyte system that recognizes them as foreign bodies). Consequently, the concepts of targeted therapy and controlled delivery were not fully embraced due to the conjunction of raw materials and active principles only in accordance to their molecular composition. In detail, the design of hydrophilic nanosystems evokes the use of water-soluble cargo, showing the criticism of the undesired rapid diffusion of hydrophilic molecules in biological medium, which in turn reduces pharmacological or imaging activity at the target site. On the other side, the hydrophobic materials are generally non-responsive towards tissue integration and their accurate biodistribution without adverse or toxic consequences over time represents a questionable point, even if their use in biomedical fields as platforms to deliver lipid organic molecules, substrates to control protein adsorption, cellular interaction and bacterial growth and diagnostic tools[2-4].

In addition, the delivery efficiency of the active principles, avoiding under- and over-dosing, has to be addressed towards the preservation of the nanosystem biocompatibility. Nowadays, tissue engineering demands nanocarriers characterized by cell selectivity and tunable pharmacokinetic. These needs lead to improve the performance of the biomaterials through the introduction of novel and specific moieties on the nano-backbone, such as aminoacids, peptides and chemical groups, for precise coupling, tunable drug release and cell recognition sites. Similarly, theranostic applications have focused their efforts in the design of smart functionalized nanovectors through the chemical grafting of biomarkers, therapeutic agents or targeting molecules. The screening and early detection of damaged cells is undoubtedly the main goal towards an effective imaging and therapeutic treatment of different disorders. For these reasons, the modification of the starting materials, such as polymers, inorganic particles, dendrimers and peptides, is a pivotal approach to achieve the discussed aims and propose new innovative systems. Indeed, several properties, such as the hydrophilicity, hydrophobicity, superficial charge and degradation time can be tuned by surface functionalization. The full potential of the nanomaterials can be expressed through the rationale study of the molecular structures and the physico-chemical interconnection with peculiar biomolecules, that represent the basis for the novel improvement strategies of the delivery performance[5]. 


\subsection{Functionalization strategies: the rationale}

The functionalization of nanomaterials can be defined as the grafting of active-functional groups pre-synthesis (structural functionalization) or post-synthesis (surface functionalization or decoration) to enhance the properties, arrange components that would otherwise not be linked together and hit the target with high precision[6-8]. This approach is commonly recognized as the strategy to tune and control the interactions between biomaterials and tissues to optimize the therapeutic effects and disease diagnostics[9, 10]. The functionalization can be exploited to control size, synthesis and self-organization of nanomaterials during their formation. In this case, a crucial aspect is the preservation of the nanometric dimensions: the coupling of new moieties needs to be addressed to minimize their effects on the steric hindrance of the final system and to tune the potential aggregation or agglomeration preventing the generation of complexing groups that bind the nanosurfaces. Biomaterials, such as nanoparticles, nanogels, micelles and dendrimers can be susceptible of modifications to be selectively captured by cells, serve as carriers in drug or gene delivery, biosensing, bio-imaging, and thermal therapy[11]. In details, the modification strategies concern both inorganic and organic compounds, with a particular emphasis on polymers $[12,13]$. The study of the physical and chemical properties of the neat materials has led to the development of different functionalization routes. They can be categorized as chemical or physical functionalization (Figure 3.1). The former generates covalent bonds between the reactive moieties giving rise to a stable linker in biological environment or to a cleavable bond, responsive to $\mathrm{pH}$, temperature, specific biochemical pathways or irradiation; otherwise, the latter includes non-covalent interactions, including van der Waals, hydrogenbonding, ionic interactions and stereo- and polyelectrolyte complexation.

\section{*** Insert Figure $3.1 * * *$}

Caption: Figure 3.1. Schematic representation of the chemical and the physical functionalization.

\subsubsection{Chemical routes}

The chemical functionalization requires the presence of specific groups anchored on the reagent molecules. Mainly, a polymer and a biomolecule are involved. The techniques can be arranged according to the involved chemical groups, as showed in Figure 3.2 . 
Caption: Figure 3.2. Chemical functionalization strategies. The reactions belonging to click chemistry are labeled in green.

\subsubsection{Esterification and modification of active ester}

The condensation reaction between the carboxyl group of an acid and the hydroxyl group of an alcohol, in the presence of a catalyst, gives rise to the formation of an ester bond (Figure 3.2a). It is commonly used in the design of controlled drug release system or biologically-driven degradation of nanomaterials. Indeed, ester bond can be hydrolyzed in physiological conditions (acid, neutral and alkaline $\mathrm{pH}$ values), according to the concentration gradient of $\mathrm{H}^{+}$and $\mathrm{OH}^{-}$ ions, and through the enzymatic activity of esterase. It represents a versatile functionalization approach to modulate the release of hydrophilic molecules in biological media following a pH-sensitive kinetic or employing selective enzymesubstrate pairs. Many esters show appreciable rates of enzyme-independent hydrolysis and cellular esterases show broad substrate reactivity, declassing the cell specificity; however, this type of linker when synthetized in branched esters configuration can be promote the molecule delivery in specific cells and cellular esterases exhibit surprising selectivity toward these complex esters[14]. Moreover, active esters (i.e. group that is highly susceptible towards nucleophilic attack) can be coupled to amine moieties to form an amide, which represents one of the most versatile linkages in organic chemistry and is characterized by high stability to hydrolysis and in extreme chemical environments[15]. This stability leads to an important biological consequence: because amino acids in proteins are linked by amide bonds, proteins do not readily hydrolyze in physiological condition and at body temperature in the absence of a specific enzyme catalyst and preserve their configuration and spatial orientation.

\subsubsection{Click chemistry}

The class of selective and orthogonal reactions to define the structure and the biochemical and mechanical properties of biomolecule-polymer hybrids is commonly recognized as 'click chemistry'. As defined by Sharpless, its philosophy is based on the definition of procedures simple to perform, insensitive to moisture and oxygen and characterized by a rapid process in mild reaction conditions, high yielding, tolerance to various chemical groups and formation of stable products [16]. Click chemistry has led to define new opportunities to design bioactive, multifunctional and architecturally controlled macromolecules involving biomotifs like proteins, peptides, aminoacids, growth factors, genes, saccharides, vitamins, nucleosides, drugs[17], to stimulate specific cellular responses at the molecular level or define a controlled theranostic therapy[18, 19]. Considering the complex physico-chemical structure of the biomolecules and of the substrate materials, the applied chemistry can be defined as 'orthogonal' to each other, to avoid any side reactions when conjugating more than one kind of biomolecules. This approach is commonly used to fabricate bio-scaffolds, like polymer films, 
fibers, spheres, hydrogels and porous network, both as bulk and as nanoscale systems, and several well-known reactions comply with the "click" context. For example, copper(I)-catalyzed alkyne-azide cycloaddition (CuAAC), strain-promoted alkyne-azide cycloaddition (SPAAC), thiol chemistry, Diels-Alder reactions, oxime ligation are stereospecific reactions that can be conducted using minimal amount of solvents and can generate only inoffensive byproducts removable with non-chromatographic purification processes[20].

- $\mathrm{CuAAC}$ reaction occurs between a terminal alkyne and an azide group, forming a thermally and hydrolytically stable triazole bond (Figure 3.2b), that cannot be oxidized or reduced[21]. Moreover, thanks to the copper(I) catalysis, the chemical mechanism ensures a complete conversion and selectivity towards the 1,4-disubstituted 1,2,3-triazole and, as a result, structural ambiguities do not exist and products separation is not necessary, compared with the conventional Huisgen 1,3-dipolar cycloaddition[22]. Indeed, $\mathrm{Cu}(\mathrm{I})$ catalyst significantly decrease the activation energy of the cycloaddition from $24 \mathrm{kcal} / \mathrm{mol}$ to $11 \mathrm{kcal} / \mathrm{mol}$ and the formation of 1,5 -triazole ring is strongly disfavored. Generally, $\mathrm{CuAAC}$ is widely used to produce polymeric materials through the conjugation of biomolecules onto a polymer backbone, or the assembling of monomers already carrying bioactive moieties. For example, Wei et al.[23] have used this strategy to 'click' folic acid targeting ligands to nanoparticles composed by polyurethane, polyethylene glycol and poly( $\varepsilon$-caprolactone) functionalized with lysine- and cystine-derivatives: these nanomaterials were able to switch tumor targeting under acidic $\mathrm{pH}$ and trigger the drug release for tumor therapy and magnetic resonance imaging (MRI). Wang et al. [24] applied the click conjugation between chitosan and folate modified poly (L-lysine) dendrons to deliver siRNA into osteosarcoma cells effectively and silence the targeted gene both in vitro and in vivo. Mauri et al.[25] have used CuAAC to label thiol-sensitive drug delivery nanosystems with a chromophore and ensure their traceability in vitro.

- SPAAC reaction can be defined as the metal-free 'click' providing bio-orthogonal modification of various biomolecules in living systems or in physiological conditions solving the questioned aspect of potential drawbacks related to the toxicity of $\mathrm{Cu}(\mathrm{I})$. In this 
case, the reaction is between an azide group and a cyclooctyne (Figure 3.2c), whose ring strain ensures a significant rate acceleration to the chemical mechanism: indeed, the active energy of this ring chain reaction could decrease to $18 \mathrm{kcal} / \mathrm{mol}$ due to the bond angle deformation, compared to the use of normal unstrained alkyne[26]. However, the high efficiency of this strategy under physiological conditions is counterbalanced by the potential outcome of a regioisomeric mixture of triazoles. In a wide range of applications, this aspect is not mandatory compared to the benefits of metal-free environment: for example, Becker et al.[27] have conjugated azido-modified peptides onto nanofibers surface possessing cyclooctynes to promote stem cell neural differentiation and neurite extension; Du et al.[28] have applied SPAAC in vivo to deliver their multifunctional nanoagents composed by zinc(II)-phthalocyanine conjugated to a lipid-poly(ethylene glycol) and following modified with cyclooctyne to azide groups on tumor tissues for photothermal therapy and photoacoustic therapy.

- Thiol chemistry includes different reactions based on the interaction between a thiol group and a heteroatom (X). The radical- and light-mediated thiol-alkene (Figure 3.2d) and thiol-alkyne reactions are widely employed in tissue engineering due to their metal-free mechanism and the advantages of a photoinitiated process activated at specific times and locations: quantitative yield of a single regioselective product, high reaction rates, compatibility with environmentally benign solvents, simple and efficient purification techniques, and insensitivity to ambient oxygen or water[29, 30]. However, the request of UV source might induce potential damages to cells and tissues[31, 32]. Alternative approaches are base/nucleophile-mediated thiol-X reactions, including Michael addition, thiol-isocyanate, thiol-epoxide and thiol-halide reactions[33]. In particular, the former is a nucleophilic addition occurring between an enolate (carbanion) and an $\alpha, \beta$-unsaturated carbonyl compound and represents a solution to produce polymeric biomaterials with a large host design of branched, linear, or network polymers, without additional toxic 
concerns and to achieve polymer side-chain and end-group modification. For example, Kröger et al[34] have functionalized linear and cyclic glucose moieties with methacrylates to design glycopolymer nanoparticles by thiol-Michael cross-linking in water: these nanosystems were used to tune the cell uptake via molecular recognition in HeLa cells; Wu et al[35] have used Michael addition process to synthetize theranostic nanoagents based on folate-conjugated reducible polyethylenimine passivated carbon dots: the resulting nanomaterial was a good siRNA gene delivery carrier in lung cancer cells, where it was selectively accumulated providing a better gene silencing and anti-cancer effect; moreover the bioimaging of carbon dots ensures monitoring and tracking of the carcinomatous tissues and the therapeutic effects.

- Diels-Alder reaction is a highly selective [4+2] cycloaddition between a diene and a dienophile to form a stable cyclohexene adduct[36] (Figure 3.2e). A peculiarity of this reaction is the thermal reversibility in the temperature range $50{ }^{\circ} \mathrm{C}-150{ }^{\circ} \mathrm{C}$ that offers the opportunity to develop 'self-healing' materials[37]. Moreover, the variant indicated as 'inverse Diels-Alder tetrazine cycloadditions' can be addressed to an improved biorthogonal feature combined with fluorogenic property designing a powerful probe for bioimaging at the cellular level, as discussed by Yang et al[38].

- Imine and oxime linkages are related to the carbonyl condensation reaction between a ketone or aldehyde group and a nucleophile (Figure 3.2f). The resulting bonds are well stable in physiological environment; however, a potential limitation of oxime ligation lies in the requirement of neutral and basic condition to minimize potential oxime exchange reaction. Compared to $\mathrm{CuAAC}$ and thiol-X mechanism, the oxime ligation can be directly performed at room temperature avoiding the use of metal catalyst or UV light, and ensuring a safe application both in vitro and in vivo studies. The biorthogonal formation of oxime was discussed by Tang et al[39]: they synthetized polyethylene glycol-polylactide (PEGPLA) nanoparticles, surface-functionalized with aldehyde groups as targeting ligands and 
an oxyamine group (the artificial target) was injected into murine breast cancer cells through liposome delivery; the reaction between aldehyde and oxyamine moieties ensured an in vivo cancer targeting.

\subsubsection{Other chemical cross-linking strategies}

The chemical panorama of nanomaterial functionalization extends beyond the esterification and the click philosophy, involving other synthetic strategies to promote the self-assembling of nanosystems in situ or their surface decoration.

Schiff-base crosslinking involves the reaction of macromolecules containing alcohol, amine or hydrazide functionalities with aldehydes. The reaction usually occurs under physiological or basic conditions with aromatic amines to form a Schiff's base, which is $\mathrm{pH}$-responsive according to its chemical structure. This linkage can be generated in situ with cells, tissues and bioactive molecules and it is characterized by a better chemical stability to $\mathrm{pH}$ value changes compared to imine, oxime or hydrazone bonds, due to the mesomeric effect that reduces the electrophilicity of the original $\mathrm{C}=\mathrm{N}[40]$. Due to the mild reaction conditions, this process has been utilized to prepare cell-compatible nanocarriers for cell internalization and controlled drug delivery applications. Chen et al[41] conjugated heparosan to the anticancer drug doxorubicin via Schiff base and the resulting material, due to its amphiphilic nature, could self-assemble into nanoparticles in aqueous solution. These nanonetworks were rapidly internalized in endosome of HeLa and human pulmonary carcinoma cell lines, releasing the drug according to the acid-sensitive nature of the Schiff-base crosslinking (Figure 3.3). Instead, Aggarwal et al[42] synthetized poly(lactide)-co-glycolide-polyethylene glycol (PLGA-PEG) nanoparticles with coupling of epidermal growth factor receptor (EGFR)-specific monoclonal antibody to achieve a cell type-specific drug carrier system against pancreatic cancer.

*** Insert Figure $3.3 * * *$

Caption: Figure 3.3. Synthesis of heparosan-DOX conjugated nanoparticles using one of the Schiff-base reaction and evaluation of: nanoparticle uptake (in red) in HeLa (a1-3) and A549 (b1-3) cell line, flow cytometric quantitative 
determination of red fluorescent $\operatorname{DOX}(\mathrm{a} 4, \mathrm{~b} 4)$ and drug release profile at $\mathrm{pH} 7.4$ and 5.0 (c1). Cell nuclei were stained by DAPI (in blue). (Adapted from Chen et al. [41] [with permission of Elsevier. Copyright 2014])

Another important class of reaction is related to the ring-opening mechanisms which represent very versatile methods for polymer transformations. The nucleophilic attack on strained heterocycles, such as epoxides[43,44] and aziridines[45], enables the grafting of desired heteroatoms on the polymer backbone. Finally, multicomponent reactions (MCRs) define methods to introduce a high degree of functional complexity in a single atom modification step and include, for example, isocyanide- or non-isocyanide-based reactions and organometallic catalyzed reactions[46, 47].

\subsubsection{Physical routes}

Tunable nanoscale modifications can be also performed through non-covalent interactions, such as van der Waals and hydrogen-bonding, hydrophobic interactions, charge transfer interactions and stereo- and polyelectrolyte complexation: in these cases, the physico-chemical features of the starting materials play a leading role in the definition of the architectural structure of the final nanovehicle. Generally, these processes focus on amphiphilic materials combined with therapeutic molecules in emulsions (water/oil or oil/water) followed by the evaporation of the organic solvent and purification through dialysis, or ionic interactions between the polymers and the payload. The main advantage of the physical functionalization is the preservation of the reagent molecular structure, in particular when the functionalization involves the use of protein- or peptide-derivatives, and for this reason, this approach is usually define as non-destructive in nature and easily accessible. However, the stability of a physical grafting is limited compared to nanostructures produced via chemical functionalization and the ability to control the orientation of the adsorbed molecules is not guaranteed[48]. Physical modifications include the coating of a biomaterial surface with a specific biomimetic functional group without altering the chemical features of either: for example, collagen, 
laminin, integrin, fibronectin are commonly used as coating layers[49], and chitosan and gelatin are chosen as extracellular matrix-resembling molecules to decorate the nanosurface[50, 51], enhancing the cell adhesion and proliferation on the functionalized biomaterial. Primarily, these methods are based on the physical adsorption of the functional molecules over the substrate and they are mainly governed by Waals forces, hydrogen bond or hydrophobic interactions, which lead to consider the strategies as 'non-destructive' and easily accessible. However, the tunable orientation of the adsorbed biomolecules remains an important challenge: the physical adsorption gives rise to a random distribution of the functional moieties, not ensuring a specific orthogonal conformation in the final nanomaterial, that can represent a critical aspect in some applications[52]. Moreover, the surface coating may influence the cellular response through the surface topography, charge, and wettability. For example, Mavis et al[53] have used polycaprolactone (PCL) as a fiber-based nanoscaffold for tissue engineering applications: due to the hydrophobic nature of the polymer surface, cell adhesion was strictly limited and they have deposited calcium phosphate on the polymer obtaining an improved osteoblastic activity of the cultured cells. Campos et al[54] studied the adsorption of fibronectin on PLGA scaffolds surface developing a tailored cell recognition system that exploits the interactions with specific integrin binding sites. Musrchel et al[55] have proposed a physical adsorption of vascular endothelial growth factor (VEGF) onto poly(allylamine)-functionalized polystyrene. Other extracellular matrix-resemble compounds were investigated for the surface functionalization of nanobiomaterials to modulate the stem cell fate in vitro for a wide range of tissue engineering applications.

\subsection{The application in tissue engineering}

The successful use of rationale-modified nanomaterials is strongly dependent on their potential in vitro and in vivo studies, in terms of interactions with the biological barriers. Indeed, the release of a payload requires that the nanosystems can overcome the cellular frontiers and reach the target site. Over the past few years, a wide range of strategies have been focused on the modulation of nanomaterials size or physico-chemical properties to cross the different biological barriers, including functionalization approaches to decorate the surface or add functional characteristics. For example, using 
responsive moieties, nanostructures can be designed to be sensitive to specific internal or external stimuli, such as $\mathrm{pH}$ variations, activity of overexpressed enzymes, intracellular reductive environment, temperature changes, magnetic field or light irradiation, which enable the cell internalization via endocytosis, the penetration of tight barriers (in the case of stratum corneum), or the targeted delivery of the drugs.

Different biological barriers could require different arrangements in the synthesis of an adaptable nanosystem due to their specific composition or physico-chemical behavior: the blood brain barrier (BBB) hampers the passage of big or hydrophilic molecules into the cerebrospinal fluid and this represent a great crucial point in the development of novel therapies for most central nervous system (CNS) and brain disorders. Regarding the tumoral scenario, the enhanced permeation and retention (EPR) effect can improve the accumulation and penetration of nanostructures in the tumor microenvironment, overcoming the aspect of their low retention time due to the increase of the interstitial fluid pressure and the heterogeneous vasculature of the tumor. Another common biological barrier is the epithelium: nasally and pulmonary administration of nanosystem require to cross the stratum corneum, which is the main challenge of this approach.

\subsubsection{The cell membranes barrier}

Cell membranes can be defined as thin semi-permeable layers composed by phospholipids and embedded with proteins[56]. They are very selective and can enable the internalization of some molecules (nutrients) and prevent the access of other ones (foreign molecules). However, the main criteria ruling the diffusive transport of compounds are related to the electric charge, the polarity, and the molar mass of the molecule. The nanostructure uptake can be promoted exploiting these guidelines: indeed, positively charged nanosystems can link to the negative charged cell membrane through electrostatic interaction, the hydrophobicity can improve the nanosystem adhesion to the cell membranes and the receptor-mediated uptake represents a mechanism for selective cell internalization. In all cases, the result is an increase of the amount of internalized nanomaterial.

Gordon et al[57] designed nanogels characterized by a charge modulation activated by an enzymatic process close to a tumor microenvironment. This nanomaterial was formulated from a random copolymer obtained by pyridyl disulfide ethylmethacrylate (PDSEMA) and polyethylene glycol monomethyl ether methacrylate. The surface charge conversion was addressed installing a protease-cleavable substrate to the nanoparticle at its C-terminus that is shielded by PEG at its N-terminus (Figure 3.4). The peptide hydrolysis by metalloproteinase-9 (MMP-9) removes the PEG decoration, revealing an 'active' surface composed by amine groups that, due to the charge conversion (from neutral to positive charge) and the reduced steric stabilization, shows enhanced cell membrane interactions and a higher uptake in tumors. 
Caption: Figure 3.4. A: Representation of MMP-9-responsive nanogels and resulting mechanism of cell uptake in presence of glutathione. B: Structures of polymer nanosystem and mechanism of activation of the positively charged surface (Adapted from Gordon et al. [57] [with permission of American Chemical Society. Copyright 2018])

Indeed, the MMP-9 is a protease frequently observed in the tumor extracellular environment and the conversion from passive PEG-coated particles to active amine-decorated particles can be modulated on time and by the biological concentration of MMP-9, resulting in a MMP-9 upregulated cell uptake. Moreover, the proteolysis of the surface does not cause guest release or nanogel disassembly, until the cellular internalization, where the glutathione was able to degrade the material.

\subsubsection{The tumor environment}

The main critical points of a tumor therapy are connected to the relapses, metastasis and drug resistance developed by the cancer cells. In particular, the latter is affected by physiological and physical mechanisms that lead limited benefits in most treatments: nanosystems usually interact only with the tumor peripheral region due to the increase of the interstitial fluid pressure in the damaged microenvironment supported by the high density of cells and extracellular matrix, leaky vasculature and limited lymphatic drainage. To overcome these barriers, a good distribution and a tailored uptake are the essential aspects to be improved. Nanomaterials with a dimension in the range 50-100 nm can be transported to the entire tumor tissue and less accumulated in the spleen, increasing the half-life[58]. On the other hand, nanocarriers modulating their size according to different external stimuli are the alternatives to reach a deep distribution in the damaged sites after shrinking or swelling in response to any local stimulus[59-61]. In this case, methacrylate and acrylate groups[62, 63] or cationic polymers, such as chitosan[60] and polyethyleneimine[64], are involved.

Stefanick et al[65] have discussed a strategy for the selective targeting in myeloma cells using liposomal nanoparticles. They conjugated two peptide-receptors (VLA-4 and LPAM-1 targeting peptides) to the liposome surface, with an optimal control over the stoichiometry of targeting ligands on the nanosystem (Figure 3.5). The resulting nanostructure was able to link to the cells expressing both VLA-4 and LPAM-1 receptors, simultaneously and negligible uptake by cell lines expressing only one or none of the two receptors, discriminating between the desired target cells and the healthy areas. The strategy is based on the pre-functionalization of the nanosystem: they synthetized the peptide-lipid conjugates using the Fmoc chemistry on a solid support (amide or resin) prior to liposome preparation, in order to ensure the reproducibility and eliminate the use of coupling agents post-nanosynthesis. 
Caption: Figure 3.5. Liposomes functionalization with peptides VLA-4 and LPAM-1 and representation of the selective cell internalization regarding cells expressing both VLA-4 and LPAM-1 receptors (Adapted from Stefanick et al. [65] [with permission of Royal Society of Chemistry. Copyright 2019])

Biscaglia et al[66] have proposed a targeting strategy based on the functionalization of gold nanoparticles with cyclic RGD-modified PEG. The conjugation between them was performed using different oligolysine spacers. The rationale combination of the peptide moiety and the plasmonic nature of the nanosystem ensured, respectively, an efficient targeting towards colorectal cancer cells overexpressing $\alpha_{v} \beta_{3}$ integrin, and the cancer imaging, monitoring the therapeutic effect.

\subsubsection{The blood brain barrier}

The blood brain barrier (BBB) is a highly semipermeable membrane composed by endothelial cells and pericytes connected by tight junctions which selectively exclude most molecules, including many therapeutic drugs, from entering the central nervous system (CNS). The membrane is composed by heparan sulfate proteoglycans, laminin, collagen (type IV) and other extracellular matrix proteins. Moreover, an electrical resistance (around 1500-2000 $\Omega \mathrm{cm}^{2}$ ) between the endothelial cells contributes to regulating the penetration of small molecules, such as water, some lipid-soluble compounds and gases by passive diffusion and blocks the passage of large molecules characterized by high electric charge, polarity and hydrophilicity, which has to rely on specific proteins via active transport routes to cross the BBB. Referring to this scenario, in the last years, a convenient method to realize the transport of drugs focuses on the temporary disruption of the extracellular matrix protein to improve the BBB permeability[67, 68]. However, the use of osmotic pressure, microbubbles and ultrasound is a risk because could damage the BBB integrity and an uncontrolled diffusion of both therapeutic compounds and unwanted toxins would take place[69-71]. For these reasons, functionalized nanostructures represent a potential method to minimize the BBB side-effects[72]. Nanoparticles are decorated with nucleic acid[73], peptides[74], proteins[75], antibodies[76], antiretroviral or anticancer molecules[77, 78], following the previously discussed chemical or physical routes. Materials commonly used are polymers like polybutylcyanoacrylate (PBCA), PLGA and PLA, liposomes and inorganic composite materials such as gold, silver and zinc oxide nanoparticles[72, 79, 80].

For example, Cox et al[75] proposed a strategy for a multiple-functionalization of polyisoprene (PI) nanoparticles: via nitroxide-mediated polymerization (NMP), they chemically linked adenosine as a model drug, TEMPO-rhodamine as a labeling molecule and maleimide groups at the nanoparticles surface for the recognition of BBB-crossing proteins. This surface modification resulted in a significant enhancement of the nanoparticle passage through an in vitro BBB model 
due to the specific conjugation with the protein corona, performed by the selective interaction between maleimide and the proteins having a free cysteine residue (bovine serum albumin, $\alpha 2$-macroglobulin or Fetuin A), as schematically reported in Figure 3.6A. In this case, the surface functionalization with BBB-targeting proteins represents a rational approach to enhance the nanomaterial crossing through the BBB, opening new perspectives in the therapeutic treatments of neurodegenerative pathways.

Moreover, an efficient therapy requires to reach the biological target, limiting undesirable off-target activities including the immunological response, related to the nanomaterial uptake by macrophage/microglia cells. Indeed, the internalization of drug delivery nanosystems within the macrophage/microglia cytosol considerably reduces the amount of active principle available for the curative aims.

Mauri et al[81] have recently investigated the potential effects of primary amine groups in the modulation of microglia uptake. Nanogels composed by polyethylene glycol-polyethyleneimine (PEG-PEI) were decorated with two different strategies: the first one, via nucleophilic substitution, decorating the nanosystems with terminal - $\mathrm{NH}_{2}$ groups; the second one, through PEGylation combined with amine groups. The in vitro studies demonstrated that the only presence of amine moieties allows less phagocytosis, whereas the nanosystem functionalization with amine-modified PEG led to the minimal cellular internalization (Figure 3.6B). In addition, the two nanostructures were characterized by different surface properties (topography and hydration degree) and the different protonation state could be responsible of the decreased cell adhesion and the activation of different microglia response. This scenario proposes an alternative and challenging approach, based on the nanosurface grafting of specific chemical groups to modulate the cell uptake through the interaction cell membrane/receptors-chemical groups, and design a target therapy.

$$
\text { **** Insert Figure } 3.6 * * *
$$

Caption: Figure 3.6. Functionalization strategies for the central nervous system scenario. A: Functionalization of nanoparticles with maleimide group to improve the crossing of BBB through the selective conjugation between maleimide and recognition proteins. (Adapted from Cox et al. [75] [with permission of Elsevier. Copyright 2019]). B: Nanogel surface decoration with differen amine-based approach to modulate the microglia uptake (Adapted from Mauri et al. [81] [with permission of Elsevier. Copyright 2020])

\subsection{The application in theranostic}


The development of theranostic nanoprobes is a focus of the latest researches. Indeed, the functionalization of nanomaterials provides a targeted accumulation in tumors tissue through the enhanced permeability and retention (EPR) effect, which helps to identify the injured area and confine the drug delivery[7, 82]. The tumoral conditions are characterized by an increased level of different growth factors (endothelial and basic fibroblast) compared to a well-being state of the cells, and this differentiation supports the targeting strategies. Also in this case, the conjugation of proteins and peptides over a nanostructure appears as the most promising solution, in particular using the cross-linking between maleimide and thiol groups or the coupling between amine and carboxyl groups.

Ligands of lipoprotein receptor-related protein (LRP), such as Angiopep-2, can be conjugated to PEG-PCL nanoparticles designing a system that can be selectively accumulated in glioma tissue due to EPR effect, bypassing the BBB, in higher concentration than the non-modified systems, as discussed by Xin et al[83].

Moreover, multiple orthogonal bioconjugation strategies have been developed to bond peptide moieties, labeling molecules and therapeutic drugs to the same construct. Cano-Cortes et al[84] have recently performed a triple functionalization of polyvinylpyrrolidone (PVP) nanoparticles, using the peptide coupling strategy (reaction between the peptide terminal $-\mathrm{NH}_{2}$ and the nanoparticle - $\mathrm{COOH}$ groups) to link the CRGDK peptide, the hydrazone bond to conjugate doxorubicin (DOX), and the orthogonal reactivity of Dde protecting groups to graft the marker Cy7. The resulting formulated nanosystems were able to target triple negative breast cancer (TNBC) cells overexpressing neuropilin-1 thanks to the selective interaction of the grafted peptide and release the drug through the cleavability of the hydrazone linker in acid $\mathrm{pH}$, like the one in tumor cells. As explained by the authors, the rationale choice of the multi-functionalization strategies provides the possibility to graft other molecules presenting the same reactive chemical groups, defining the versatility of the proposed nanotheranostic device.

Finally, another innovative method regards the design of smart functionalized 2D nanomaterials[85] aimed to an application in photothermal therapy (PTT) as well as synergistic cancer therapy. Generally, these materials are composed by graphene and its derivatives, metallic compounds, transition metals, black phosphorous, specific moieties such as carbides, nitrides or carbonitrides and polymers. Biomolecules, fluorescent dyes, radioisotopes or drugs can be conjugated to the $2 \mathrm{D}$ substrate via chemical bonding, physical adsorption, hydrophobic or electrostatic interactions, to provide an improvement in properties such as biocompatibility, non-toxicity, high payload binding, targeted tumor accumulation and cellular uptake. About the polymers, natural and synthetic chains are used as coating agent to modulate the hydrophilic behavior, the cell internalization, the compatibility and degradability in biological environment. Dextran, cellulose, chitosan, PEG, PEI, PVP, polyacrylic acid and polyvinyl alcohol are commonly chosen to achieve these aims[86-88]. Instead, decoration with noble metals or metal oxides, including palladium, platinum, silver and gold, guarantees a surface plasmon resonance absorption which in turn increases the photothermal conversion efficiencies. The radioisotopic 
labelling can be used in both PTT and positron emission tomography (PET) to synthetize specific radio-nucleotide labeling for imaging and diagnosis in cancer therapy: for example, PEGylated substrates were functionalized with ${ }^{131} \mathrm{I}$ radio-nucleotide, showing strong NIR absorbance and radio-ionization effect, resulting in a potential nanodevice for combined radio-photothermal therapy[89]; whereas, the use of ${ }^{64} \mathrm{Cu}$ radioisotope increased the performance in PET imaging and radiotherapy[90].

\section{Conclusions}

Tissue engineering and theranostic require nanomaterials satisfying different specific needs, from the biocompatibility to the selective targeting. The only smart combination of different compounds is not enough to design a powerful nanodevice able to meet all the requirements: generally, specific moieties are essential to perform desired nanomaterial-cell interactions, to promote a controlled release of therapeutics or to ensure the monitoring of the disease over time. For these reasons, functionalization strategies represent the adequate tool to design tailored nanostructures grafting biomolecules, such as peptides, proteins and drugs, or chemical groups. In particular the versatility of the functionalization methods lies in the potential choice between chemical or physical routes to achieve a rational modification of nanomaterials, complying with the characteristic of the added functionality: chemical strategies lead to the formation of covalent linkages that can be stable or cleavable under specific biological conditions, whereas physical methods are basically focused on weak interactions that do not affect the conformation and the chemical structure of the introduced moieties. Delivery performances can be successfully improved through the use of these techniques, overcoming the main constraint in the biological applications and tackling new challenge to optimize the therapeutic effects.

\section{[Chapter References]}

[1] J.O. Martinez, B.S. Brown, N. Quattrocchi, M. Evangelopoulos, M. Ferrari, E. Tasciotti, Chin Sci Bull, 57 (2012) 3961-3971.

[2] S. Kalepu, V. Nekkanti, Acta Pharmaceutica Sinica B, 5 (2015) 442-453.

[3] A.C. Yu, L.M. Stapleton, J.L. Mann, E.A. Appel, 10 - Self-assembled biomaterials using host-guest interactions, in: H.S. Azevedo, R.M.P. da Silva (Eds.) Self-assembling Biomaterials, Woodhead Publishing, 2018, pp. 205-231.

[4] E.J. Falde, S.T. Yohe, Y.L. Colson, M.W. Grinstaff, Biomaterials, 104 (2016) 87-103.

[5] P. Sengupta, B.L.V. Prasad, Acs Omega, 3 (2018) 4242-4251.

[6] J. Jeevanandam, A. Barhoum, Y.S. Chan, A. Dufresne, M.K. Danquah, Beilstein J Nanotech, 9 (2018) 1050-1074. 
[7] R. Thiruppathi, S. Mishra, M. Ganapathy, P. Padmanabhan, B. Gulyas, Adv Sci, 4 (2017).

[8] R.A. Bohara, N.D. Thorat, S.H. Pawar, Rsc Adv, 6 (2016) 43989-44012.

[9] G.S. Wu, P.H. Li, H.Q. Feng, X.M. Zhang, P.K. Chu, J Mater Chem B, 3 (2015) 20242042.

[10] E. Mauri, G. Perale, F. Rossi, Acs Applied Nano Materials, 1 (2018) 6525-6541.

[11] S. Parveen, R. Misra, S.K. Sahoo, Nanomedicine: Nanotechnology, Biology and Medicine, 8 (2012) 147-166.

[12] E. Mauri, S. Papa, M. Masi, P. Veglianese, F. Rossi, Expert Opin Drug Del, 14 (2017) 1305-1313.

[13] W.J. Yang, L. Liang, X. Wang, Y. Cao, W. Xu, D. Chang, Y. Gao, L. Wang, Biomater Sci-Uk, 7 (2019) 247-261.

[14] L. Tian, Y.L. Yang, L.M. Wysocki, A.C. Arnold, A. Hu, B. Ravichandran, S.M. Sternson, L.L. Looger, L.D. Lavis, P Natl Acad Sci USA, 109 (2012) 4756-4761.

[15] R.J. Ouellette, J.D. Rawn, 12 - Amines and Amides, in: R.J. Ouellette, J.D. Rawn (Eds.) Principles of Organic Chemistry, Elsevier, Boston, 2015, pp. 315-342.

[16] H.C. Kolb, M.G. Finn, K.B. Sharpless, Angewandte Chemie - International Edition, 40 (2001) 2004-2021.

[17] L.S. Nair, C.T. Laurencin, Prog Polym Sci, 32 (2007) 762-798.

[18] L.L. Hench, J.M. Polak, Science, 295 (2002) 1014-1017.

[19] Y. Zou, L. Zhang, L. Yang, F. Zhu, M. Ding, F. Lin, Z. Wang, Y. Li, J Control Release, 273 (2018) 160-179.

[20] H.C. Kolb, K.B. Sharpless, Drug Discov Today, 8 (2003) 1128-1137.

[21] E. Haldón, M.C. Nicasio, P.J. Pérez, Org Biomol Chem, 13 (2015) 9528-9550.

[22] R. Huisgen, Pure and Applied Chemistry, 1989, pp. 613.

[23] J. Wei, X. Shuai, R. Wang, X. He, Y. Li, M. Ding, J. Li, H. Tan, Q. Fu, Biomaterials, 145 (2017) 138-153.

[24] F. Wang, J.D. Pang, L.L. Huang, R. Wang, D. Li, K. Sun, L.T. Wang, L.M. Zhang, Int J Nanomed, 13 (2018) 857-875.

[25] E. Mauri, P. Veglianese, S. Papa, A. Mariani, M. De Paola, R. Rigamonti, Giulia M.F. Chincarini, I. Vismara, S. Rimondo, A. Sacchetti, F. Rossi, Rsc Adv, 7 (2017) 30345-30356.

[26] N.J. Agard, J.A. Prescher, C.R. Bertozzi, J Am Chem Soc, 126 (2004) 15046-15047.

[27] L.A. Smith Callahan, S. Xie, I.A. Barker, J. Zheng, D.H. Reneker, A.P. Dove, M.L. Becker, Biomaterials, 34 (2013) 9089-9095. 
[28] L. Du, H. Qin, T. Ma, T. Zhang, D. Xing, Acs Nano, 11 (2017) 8930-8943.

[29] A.B. Lowe, Polym Chem-Uk, 5 (2014) 4820-4870.

[30] M.J. Kade, D.J. Burke, C.J. Hawker, J Polym Sci Pol Chem, 48 (2010) 743-750.

[31] S.-L. Yu, S.-K. Lee, Molecular \& Cellular Toxicology, 13 (2017) 21-28.

[32] T.M. Rünger, Journal of Investigative Dermatology, 127 (2007) 2103-2105.

[33] C.E. Hoyle, A.B. Lowe, C.N. Bowman, Chem Soc Rev, 39 (2010) 1355-1387.

[34] A.P.P. Kröger, M.I. Komil, N.M. Hamelmann, A. Juan, M.H. Stenzel, J.M.J. Paulusse, Acs Macro Lett, 8 (2019) 95-101.

[35] Y.-F. Wu, H.-C. Wu, C.-H. Kuan, C.-J. Lin, L.-W. Wang, C.-W. Chang, T.-W. Wang, Sci Rep-Uk, 6 (2016) 21170.

[36] M.A. Tasdelen, Polym Chem-Uk, 2 (2011) 2133-2145.

[37] Y. Zhang, A.A. Broekhuis, F. Picchioni, Macromolecules, 42 (2009) 1906-1912.

[38] J. Yang, J. Šečkutė, C.M. Cole, N.K. Devaraj, Angewandte Chemie International Edition, 51 (2012) 7476-7479.

[39] L. Tang, Q. Yin, Y. Xu, Q. Zhou, K. Cai, J. Yen, L.W. Dobrucki, J. Cheng, Chem Sci, 6 (2015) 2182-2186.

[40] Z. Zhang, C. He, X. Chen, Materials Chemistry Frontiers, 2 (2018) 1765-1778.

[41] J.-X. Chen, W. Liu, M. Zhang, J.-H. Chen, Int J Pharm, 473 (2014) 493-500.

[42] S. Aggarwal, S. Gupta, D. Pabla, R.S.R. Murthy, Cancer Nanotechnology, 4 (2013) $145-157$

[43] A. Pikabea, J. Forcada, J Polym Sci Pol Chem, 55 (2017) 3573-3586.

[44] M.O.P. Kara, A.K. Ekenseair, Biomacromolecules, 18 (2017) 1473-1481.

[45] R. Akhtar, S.A.R. Naqvi, A.F. Zahoor, S. Saleem, Mol Divers, 22 (2018) 447-501.

[46] Y. Pan, L.J. Ma, S. Lin, Y.F. Zhang, B.W. Cheng, J.Q. Meng, J Mater Chem A, 4 (2016) 15945-15960.

[47] M.A. Mironov, I.D. Shulepov, V.S. Ponomarev, V.A. Bakulev, Colloid Polym Sci, 291 (2013) 1683-1691.

[48] D. Rana, K. Ramasamy, M. Leena, C. Jiménez, J. Campos, P. Ibarra, Z.S. Haidar, M. Ramalingam, Biotechnology Progress, 32 (2016) 554-567.

[49] A. Ibara, H. Miyaji, B. Fugetsu, E. Nishida, H. Takita, S. Tanaka, T. Sugaya, M. Kawanami, J Nanomater, 2013 (2013) 11.

[50] S. Bierbaum, V. Hintze, D. Scharnweber, Biomatter, 2 (2012) 132-141.

[51] L. Yang, M. Yaseen, X. Zhao, P. Coffey, F. Pan, Y. Wang, H. Xu, J. Webster, J.R. Lu, Biomed Mater, 10 (2015) 025003. 
[52] D. Rana, K. Ramasamy, M. Leena, C. Jimenez, J. Campos, P. Ibarra, Z.S. Haidar, M. Ramalingam, Biotechnology Progress, 32 (2016) 554-567.

[53] B. Mavis, T.T. Demirtas, M. Gumusderelioglu, G. Gunduz, U. Colak, Acta Biomater, 5 (2009) 3098-3111.

[54] D.M. Campos, K. Gritsch, V. Salles, G.N. Attik, B. Grosgogeat, Bioresearch Open Acc, 3 (2014) 117-126.

[55] F. Murschel, A. Zaimi, S. Noel, M. Jolicoeur, G. De Crescenzo, Biomacromolecules, 16 (2015) 3445-3454.

[56] S.J. Singer, G.L. Nicolson, Science, 175 (1972) 720-+.

[57] M.R. Gordon, B. Zhao, F. Anson, A. Fernandez, K. Singh, C. Homyak, M. Canakci, R.W. Vachet, S. Thayumanavan, Biomacromolecules, 19 (2018) 860-871.

[58] N. Hoshyar, S. Gray, H.B. Han, G. Bao, Nanomedicine-Uk, 11 (2016) 673-692.

[59] M.H. Zan, J.J. Li, S.Z. Luo, Z.S. Ge, Chem Commun, 50 (2014) 7824-7827.

[60] C.Y. Ju, R. Mo, J.W. Xue, L. Zhang, Z.K. Zhao, L.J. Xue, Q.N. Ping, C. Zhang, Angew Chem Int Edit, 53 (2014) 6253-6258.

[61] J.C. Cuggino, E.R.O. Blanco, L.M. Gugliotta, C.I.A. Igarzabal, M. Calderon, J Control Release, 307 (2019) 221-246.

[62] H.Z. Deng, K. Song, J.H. Zhang, L.D. Deng, A.J. Dong, Z.H. Qin, Chem Commun, 54 (2018) 3014-3017.

[63] H. Yang, Q. Wang, Z.F. Li, F.Y. Li, D. Wu, M. Fan, A.B. Zheng, B. Huang, L. Gan, Y.L. Zhao, X.L. Yang, Nano Lett, 18 (2018) 7909-7918.

[64] H. Yim, S.J. Park, Y.H. Bae, K. Na, Biomaterials, 34 (2013) 7674-7682.

[65] J.F. Stefanick, D.T. Omstead, T. Kiziltepe, B. Bilgicer, Nanoscale, 11 (2019) 44144427.

[66] F. Biscaglia, G. Ripani, S. Rajendran, C. Benna, S. Mocellin, G. Bocchinfuso, M. Meneghetti, A. Palleschi, M. Gobbo, ACS Applied Nano Materials, 2 (2019) 6436-6444.

[67] N. McDannold, C.D. Arvanitis, N. Vykhodtseva, M.S. Livingstone, Cancer Res, 72 (2012) 3652-3663.

[68] S.M. Stamatovic, R.F. Keep, A.V. Andjelkovic, Curr Neuropharmacol, 6 (2008) 179192.

[69] R.K. Upadhyay, Biomed Res Int, (2014).

[70] S.K. Wu, P.C. Chu, W.Y. Chai, S.T. Kang, C.H. Tsai, C.H. Fan, C.K. Yeh, H.L. Liu, Sci Rep-Uk, 7 (2017).

[71] P.C. Chu, W.Y. Chai, C.H. Tsai, S.T. Kang, C.K. Yeh, H.L. Liu, Sci Rep-Uk, 6 (2016). 
[72] Y.Q. Zhou, Z.L. Peng, E.S. Seven, R.M. Leblanc, J Control Release, 270 (2018) 290303.

[73] H. Peluffo, U. Unzueta, M.L. Negro-Demontel, Z.K. Xu, E. Vaquez, N. Ferrer-Miralles, A. Villaverde, Biotechnol Adv, 33 (2015) 277-287.

[74] J.A. Loureiro, B. Gomes, G. Fricker, M.A.N. Coelho, S. Rocha, M.C. Pereira, Colloid Surface B, 145 (2016) 8-13.

[75] A. Cox, D. Vinciguerra, F. Re, R. Dal Magro, S. Mura, M. Masserini, P. Couvreur, J. Nicolas, Eur J Pharm Biopharm, 142 (2019) 70-82.

[76] Y. Monsalve, G. Tosi, B. Ruozi, D. Belletti, A. Vilella, M. Zoli, M.A. Vandelli, F. Forni, B.L. Lopez, L. Sierra, Nanomedicine-Uk, 10 (2015) 1735-1750.

[77] S.D. Mahajan, I. Roy, G.X. Xu, K.T. Yong, H. Ding, R. Aalinkeel, J.L. Reynolds, D.E. Sykes, B.B. Nair, E.Y. Lin, P.N. Prasad, S.A. Schwartz, Curr Hiv Res, 8 (2010) 396-404.

[78] W. Tang, W.P. Fan, J. Lau, L.M. Deng, Z.Y. Shen, X.Y. Chen, Chem Soc Rev, 48 (2019) 2967-3014.

[79] J.B. Xie, Z.Y. Shen, Y. Anraku, K. Kataoka, X.Y. Chen, Biomaterials, 224 (2019).

[80] T.T. Zhang, W. Li, G.M. Meng, P. Wang, W.Z. Liao, Biomater Sci-Uk, 4 (2016) 219229.

[81] E. Mauri, P. Veglianese, S. Papa, A. Rossetti, M. De Paola, A. Mariani, Z. Posel, P. Posocco, A. Sacchetti, F. Rossi, Colloids and Surfaces B: Biointerfaces, 185 (2020) 110574.

[82] H. Maeda, Advances in Enzyme Regulation, 41 (2001) 189-207.

[83] H. Xin, X. Jiang, J. Gu, X. Sha, L. Chen, K. Law, Y. Chen, X. Wang, Y. Jiang, X. Fang, Biomaterials, 32 (2011) 4293-4305.

[84] M.V. Cano-Cortes, S.A. Navarro-Marchal, M.P. Ruiz-Blas, J.J. Diaz-Mochon, J.A. Marchal, R.M. Sanchez-Martin, Nanomedicine: Nanotechnology, Biology and Medicine, 24 (2020) 102120.

[85] C. Murugan, V. Sharma, R.K. Murugan, G. Malaimegu, A. Sundaramurthy, J Control Release, 299 (2019) 1-20.

[86] G.Y. Liu, J.H. Zou, Q.Y. Tang, X.Y. Yang, Y.W. Zhang, Q. Zhang, W. Huang, P. Chen, J.J. Shao, X.C. Dong, Acs Appl Mater Inter, 9 (2017) 40077-40086.

[87] S.X. Shi, T. Liu, C. Liang, T. Barnhart, Z. Liu, W.B. Cai, J Nucl Med, 56 (2015).

[88] L. Cheng, J.J. Liu, X. Gu, H. Gong, X.Z. Shi, T. Liu, C. Wang, X.Y. Wang, G. Liu, H.Y. Xing, W.B. Bu, B.Q. Sun, Z. Liu, Adv Mater, 26 (2014) 1886-1893.

[89] L. Chen, X.Y. Zhong, X. Yi, M. Huang, P. Ning, T. Liu, C.C. Ge, Z.F. Chai, Z. Liu, K. Yang, Biomaterials, 66 (2015) 21-28. 
[90] L. Cheng, S.D. Shen, S.X. Shi, Y. Yi, X.Y. Wang, G.S. Song, K. Yang, G. Liu, T.E. Barnhart, W.B. Cai, Z. Liu, Adv Funct Mater, 26 (2016) 2185-2197. 


\section{HR-MAS NMR Spectroscopy: novel technologies to measure delivery performances \\ Franca Castiglione, Andrea Mele}

\section{Introduction}

Understanding the transport phenomena at a molecular level is of key importance to explain and regulate macroscopic processes such as controlled-release and safe delivery of drugs. Among all the experimental techniques, Nuclear Magnetic Resonance Spectroscopy (NMR) is widely used to study structure, rotational and translational dynamics of small-drug molecules in liquid state solution ${ }^{1-6}$. High resolution magic angle spinning (HR-MAS) is a further recent ${ }^{7}$ advancement $^{-}$ of the NMR spectroscopic technique as it provides a direct analysis of heterogeneous soft materials ${ }^{8,9}$ such as gels $^{10}$, polymers ${ }^{11}$ and cells ${ }^{12-14}$ characterized by low molecular mobility. Indeed, HR-MAS NMR technique is based on spinning the sample at the magic angle (MAS) to reduce resonance line broadening caused by anisotropic interactions inherent to semi-solid materials and enforced to small molecules encapsulated in gel systems. Thus, high resolution (HR) signals are observed in heterogeneous semi-solid materials and biological samples. Several HR approaches, in particular pulse field gradient spin echo (PGSE) experiments, are used to study the motion regime of small drugs entrapped in swollen polymers or hydrogels.

This chapter introduces the novel field of HR-MAS NMR starting with the basic theoretical principles of the NMR Spectroscopy, the MAS approach and a short summary of the experimental procedure in section 1. The PGSE theory combined with MAS, and concepts on the motion regime are introduced in section 2. Showcases of the application of MAS PFG NMR are presented in Section 3. They include the study of the drug motion regime in swollen cyclodextrinbased polymers (cyclodextrin nanosponges, section 3.1) and diffusion study of small drugs loaded in hydrogel carbomeragarose polymers in section 3.2. In this case a relation between the drug motion and drug-polymer or drug-drug interaction is discussed.

\section{High Resolution Magic angle spinning NMR}

\subsection{Theory}

The general spin Hamiltonian which describes an NMR experiment ${ }^{15-18}$ is given by:

$$
H=H_{Z}+H_{R F}+H_{\text {int }}
$$

The first two terms $H_{Z}$ and $H_{R F}$ accounts for external interactions between the nuclear spins and the external magnetic fields. $H_{z}$ is the Zeeman term, which describes the interaction between a nuclear magnetic moment $\mu$ and the external static magnetic field $\mathrm{B}_{0}$.

$$
H_{Z}=-\mu \cdot B_{0}
$$

$\mu$ is proportional to the nuclear spin operator $I$ and the magnetogyric ratio $\gamma$ as $\mu=\gamma \hbar I$ with $\hbar=\frac{h}{2 \pi}$. The Zeeman interaction occurs only with nuclei having spin $I>0$ and yields $2 I+1$ energy levels. Spin transitions between different energy levels are induced by the application of a time-varying orthogonal magnetic field $\mathrm{B}_{1}$ in the radio-frequency region (10 MHz-1 GHz). The RF Hamiltonian $H_{R F}$ is expressed by:

$H_{R F}=-\frac{\hbar \hbar B_{1}}{2}\left(I_{+} e^{-i \omega t}+I_{-} e^{i \omega t}\right)$

where $I+$ and $I$ - are the conventional spin raising and lowering operators respectively.

The 'internal' spin Hamiltonian $H_{\text {int }}$ represents the interaction of the nuclear spin with its surroundings and contains relevant structural and dynamic information. $H_{\text {int }}$ is expressed by the following terms:

$$
H_{\text {int }}=H_{C S}+H_{D}^{I S}+H_{D}^{I I}+H_{Q}
$$


$H_{C S}$ is the chemical shielding Hamiltonian, which accounts for the modification of the magnetic field at the nucleus due to the surrounding electrons as they, also, have magnetic moments affected by the external field $\mathrm{B}_{0}$. The chemical shielding Hamiltonian is given by:

$$
H_{C S}=\left(\omega_{0} \sigma_{i s o}+\frac{\omega_{0} \delta_{\sigma}}{2}\left[\left(3 \cos ^{2} \vartheta-1\right)-\eta_{\sigma} \operatorname{sen}^{2} \vartheta \cos 2 \phi\right]\right) \cdot I_{z}
$$

The term $\sigma_{i s o}$ is the isotropic chemical shielding tensor, $\omega_{0}$ is the Larmor frequency of the nucleus and $\delta_{\sigma}$ is the anisotropic term. The second-rank tensor $\sigma_{i s o}$, its anisotropy and the asymmetry parameter $\eta_{\sigma}$ are most conveniently represented by a tensor $\sigma$ in the principal axis system (PAS), which is an axis frame defined in such a way that the symmetric part of the shielding tensor is diagonal, and the principal values of the shielding tensor can be given as ${ }^{15,19}$ :

$$
\begin{aligned}
& \sigma_{i s o}=\frac{1}{3}\left(\sigma_{x x}^{P A S}+\sigma_{y y}^{P A S}+\sigma_{z z}^{P A S}\right) \\
& \delta_{\sigma}=\sigma_{z z}^{P A S}-\sigma_{i s o} \\
& \eta_{\sigma}=\frac{\sigma_{x x}^{P A S}-\sigma_{y y}^{P A S}}{\delta}
\end{aligned}
$$

The angles $\vartheta$ and $\phi$ determine the orientation of the principal axis system of the tensorial anisotropic interaction with respect to the static magnetic field.

The terms $H_{D}^{I S}$ and $H_{D}^{I I}$ describe the direct magnetic interaction through space with nearby nuclear magnetic moments. This interaction may involve homonuclear I spins $\left(H_{D}^{I I}\right)$ or heteronuclear $I-S$ spins $\left(H_{D}^{I S}\right)$ and depends upon the internuclear distance according to the following equations:

$$
\begin{aligned}
& H_{D}^{I I}=-\frac{\mu_{0} \hbar}{4 \pi} \sum_{i} \sum_{j} \frac{\gamma^{2}}{r_{i j}^{3}} \frac{\left(3 \cos ^{2} \vartheta_{i j}-1\right)}{2} \cdot\left[3 I_{z}^{i} I_{z}^{j}-\left(I^{i} \cdot I^{j}\right)\right] \\
& H_{D}^{I S}=-\frac{\mu_{0} \hbar}{4 \pi} \sum_{i} \sum_{j} \frac{\gamma^{I} \gamma^{S}}{r_{i j}^{3}} \frac{\left(3 \cos ^{2} \vartheta_{i j}-1\right)}{2} \cdot 2 I_{z}^{i} S_{z}^{j}
\end{aligned}
$$

$\gamma_{I}$ and $\gamma_{S}$ represent the gyromagnetic ratios of spin $I$ and $S, \mathrm{r}_{\mathrm{ij}}$ is the magnitude of the distance vector between the interacting nuclei $\mathrm{i}$ and $\mathrm{j}$, and $\vartheta_{i j}$ is the angle between $r_{i j}$ and the $\mathrm{z}$-axis.

Nuclei with spin $I>1 / 2$ are also affected by the nuclear electric quadrupole interaction $\left(H_{Q}\right)$ with the gradient in the electric field at the nucleus. Although this is an electrical interaction it depends on the magnetic quantum number and so affects the NMR spectrum. $H_{Q}$ is represented in equation 11:

$$
H_{Q}=-\frac{e V_{z z} Q}{4 I(2 I-1) \hbar}\left[\left(3 \cos ^{2} \vartheta-1\right)-\eta_{\sigma} \operatorname{sen}^{2} \vartheta \cos 2 \phi\right] \cdot\left(3 I_{z}^{2}-I^{2}\right)
$$

$V_{z z}$ is the largest component of the electric field gradient tensor, $Q$ is the quadrupole moment of the nuclei, $h$ is the Planck constant, and $e$ is the electronic charge.

All the nuclear magnetic interactions described in eq. 5, 9-11 share a common anisotropic term of the form $\left(3 \cos ^{2} \vartheta-1\right)$ , where $\vartheta$ is the angle that indicates the molecular orientation with respect to the static magnetic field $\mathrm{B}_{0}$. Consequently, in solid and gel systems the orientational distribution of all the molecules in the sample is observed in the NMR spectra giving featureless broad lines. In order to improve spectral resolution in solids, Andrew ${ }^{20}$ and Lowe ${ }^{21}$ proposed the MAS technique based on the mechanical spinning of the sample tube around an axis inclined at an angle $\vartheta=\cos ^{-1}\left(\frac{1}{\sqrt{3}}\right)=54.74^{\circ}$ (called the magic angle) with respect to the direction of the static magnetic fileld $\mathrm{B}_{0}$. A schematic representation of the experimental setup is reported in Figure 1.

When the sample is spun at the magic angle with a spinning frequency $\omega_{r}$, the term $\left(3 \cos ^{2} \vartheta-1\right)=0$, thus the anisotropic part of the Hamiltonian $\left(H_{C S}\right)$ (third term in Eq. 5) produces only spinning sidebands due to the frequency modulation. In Eq. 5 also the isotropic term $\omega_{0} \sigma_{i s o}$ remains, leading to the isotropic chemical shifts in the spectrum. The mechanical rotation of the sample at the magic angle introduces a time modulation of the dipolar frequency (in Eq. 9, 10):

$\omega_{i j}^{D}(t)=\omega_{i j}^{D}\left\{G_{0}+G_{1}\left(\cos \left(\omega_{r} t+\phi\right)\right)+G_{2}\left(\cos \left(2 \omega_{r} t+2 \phi\right)\right)\right\}$

where $\omega_{i j}^{D}(t)=\frac{\mu_{0} \gamma_{I} \gamma_{S} \hbar}{4 \pi r_{I S}^{3}}, G_{0}=-\frac{\left(3 \cos ^{2} \vartheta-1\right)\left(3 \cos ^{2} \beta-1\right)}{4}, G_{1}=\frac{3}{4} \operatorname{sen} 2 \vartheta \operatorname{sen} 2 \beta, G_{2}=\frac{3}{4} \operatorname{sen}^{2} \vartheta_{\operatorname{sen}^{2}} \beta$.

The term $G_{0}=0$ at the magic angle, $G_{1}$ and $G_{2}$ are oscillatory terms that are averaged to zero over a single rotor period. At this point the NMR spectrum consists of a center band and a series of sidebands at multiple values of the spinning rate. These sidebands may complicate the spectrum, thus for an effective line narrowing and spectral simplification, the spinning rate must exceed the magnitude of the dipolar interactions. Magic angle orientation and spinning rate are the 
two important parameters to be adjusted for a partial or complete line narrowing effect in the experimental spectra. For samples that are in the heterogeneous/semi-solid classification, a restricted molecular motion will partially reduce the magnitude of the anisotropic interactions, so even moderate spinning speeds will produce high resolution spectra: this niche of the NMR spectroscopy is called HR-MAS.

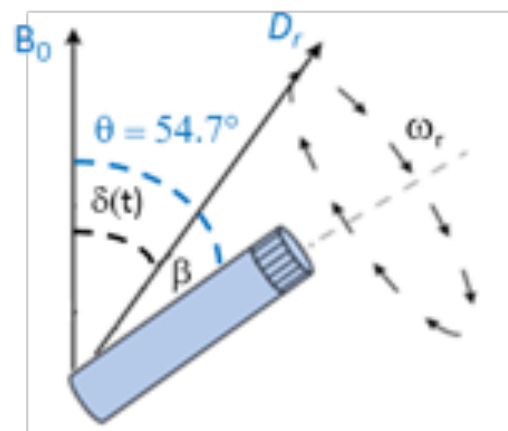

Figure 1. Schematic representation of the MAS technique, where $\theta_{\mathrm{m}}=54,7^{\circ}$ is the magic-angle, $\delta(\mathrm{t})$ is the angle between $\mathrm{B}_{0}$ and the dipolar vector, $D_{r}, \beta$ the angle between the rotation axis and $D_{R}$ and $\omega_{r}$ is the spinning frequency.

\subsection{Experimental setup}

HR-MAS experimental setup essentially consists on an NMR probe capable of magic angle orientation (MAS) and a pneumatic unit for controlling the sample spinning rate and sample insertion/ejection. HR-MAS probes ${ }^{22,23}$ are equipped with a deuterium lock channel and usually are available in double (e.g. ${ }^{1} \mathrm{H}$ and $\left.{ }^{13} \mathrm{C}\right)$ resonance modes. Moreover, these probes handle low power radio frequency (RF) and are configured with the gradient coil aligned along the magic angle enabling the access to all experimental techniques characteristic of solution-state NMR, including pulse field gradient ${ }^{24,25}$ (PFG) experiments under MAS conditions. A typical commercial HR-MAS probe is shown in Figure 2 and may be used with a conventional solution-state NMR spectrometer.

Samples for HR-MAS spectroscopy are generally packed into zirconium rotors provided with Kel-F caps and inserts (figure 2). The function of the rotor cap is twofold: firstly, to close the rotors, and secondly, to provide the driving of the rotor. Nowadays different type of rotors and spacers are available, the most commonly used is the 4 mm outer diameter rotor containing a volume of $50 \mu \mathrm{L}$ suitable for structural study. The $4 \mathrm{~mm}$ rotor designed for $12 \mu \mathrm{L}$ volume of the sample, able to minimize centrifugal effects, is particularly recommended ${ }^{26}$ for diffusion study.

For samples, such as gels, swollen polymers, lipids, where residual dipolar interactions or chemical shift anisotropy are small, sample-spinning frequencies of $2-6 \mathrm{kHz}$ are generally sufficient to obtain high-resolution spectra.

a)

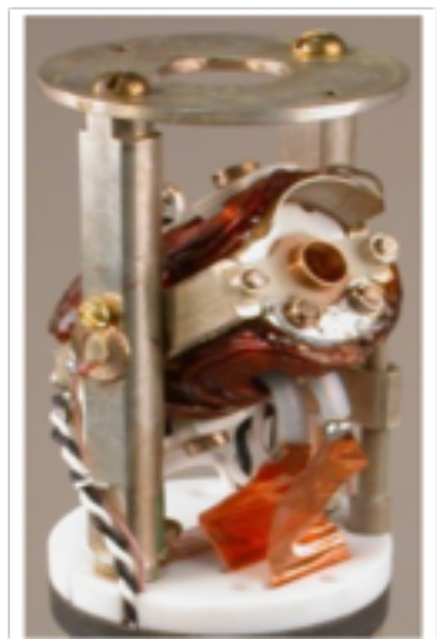

b)

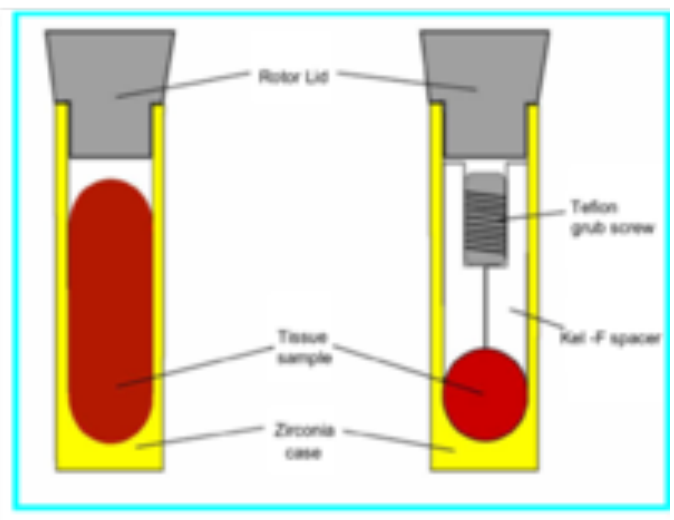

Figure 2. a) HR-MAS NMR probe oriented at the magic angle; b) A zirconia $4 \mathrm{~mm}$ (o.d.) rotor with Kel-F spacers and cups. Source: Lindon et al. 2009 [14]. Reprinted with permission 
The effect on spectral resolution obtained with ${ }^{1} \mathrm{H}$ HR-MAS technique compared with static (liquid-state-like) experiments, is reported in figure 3. The investigated system concerns a drug-like molecule, namely fluorescein encapsulated in cyclodextrin nanosponge-water-swollen polymer system especially designed for controlled drug delivery (see section 3.1). To study drug motion in confined polymer systems, diffusion experiment are usually performed following a specific molecular signal under the effect pulse field gradients. The spectrum acquired under static conditions (panel 3c) shows broad lines useless for diffusion or structural experiments. A liquid-state-like resolution is obtained for fluorescein (panel $3 \mathrm{~b}$ full spectrum) under moderate spinning speed $(4 \mathrm{KHz})$ at the magic angle.

another paradigmatic example comes from the structural investigation ${ }^{27}$ of a composite material made of polymeric hydrogel functionalized with polymer nanoparticles. This system is particularly suitable for drug delivery applications. The ${ }^{13} \mathrm{C}$ HR-MAS NMR spectrum of the swollen polymer is shown in figure 4 (panel a) and compared with high resolution ${ }^{13} \mathrm{C}$ spectrum of the monomer (4b).

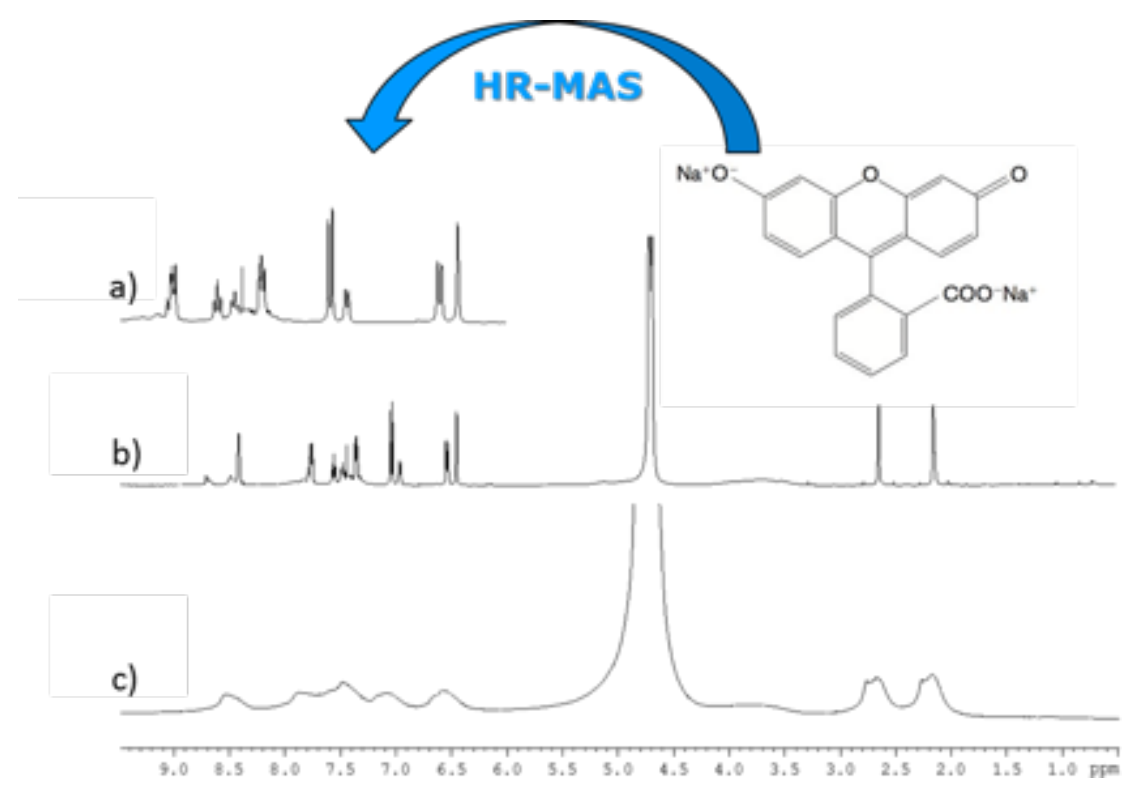

Figure 3. ${ }^{1} \mathrm{H}$ spectra of fluorescein encapsulated in nanosponge-water-swollen polymer system, c) static spectrum, b) HR-MAS spectrum spinning at $4 \mathrm{KHz}$, a) expanded aromatic region of $\mathrm{B}$ and sketch of fluorescein chemical structure.

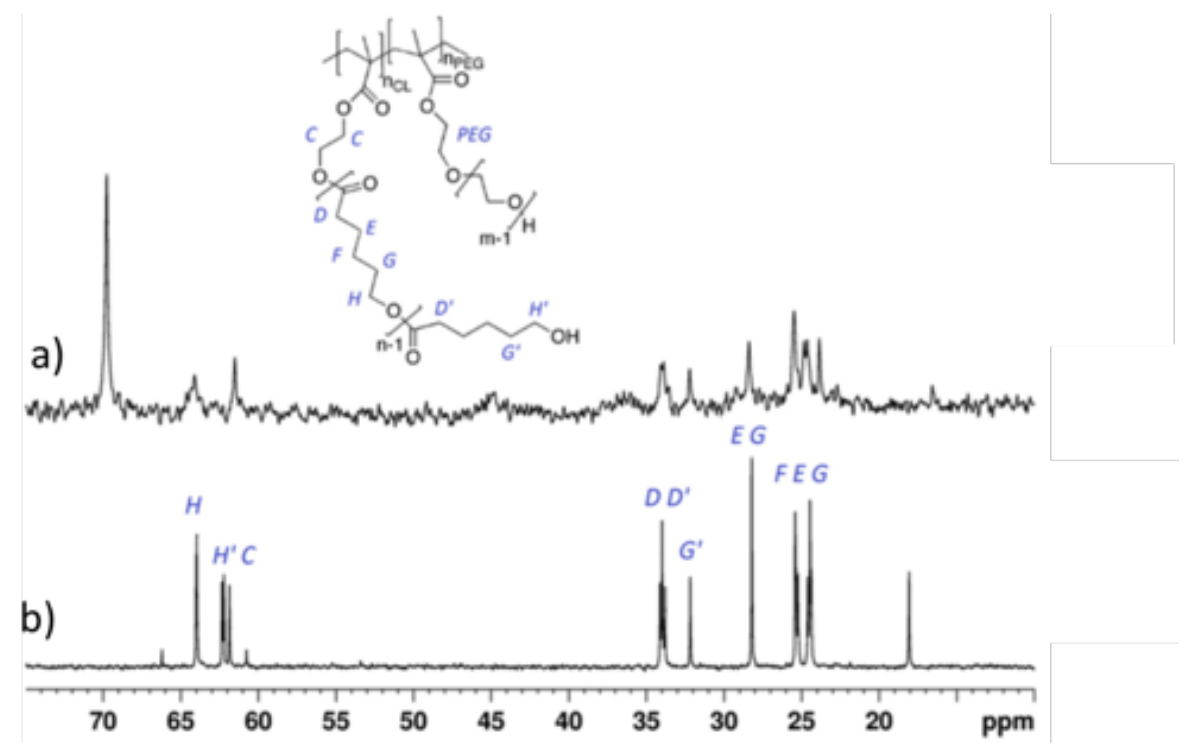

Figure 4. a) HRMAS spectrum of the f-AC polymer together with molecular formula and atom numbering; b) ${ }^{13} \mathrm{C}$ high resolution spectrum (0-85 ppm expanded region) of HEMA-CL 3 macromonomer. Source: Rossi et al. 2015 [27]. Reprinted with permission 


\section{PFG HR-MAS NMR spectroscopy}

Before describing in details the innovative approach based on using diffusion technique (pulse gradient spin echo PGSE) combined with MAS setup, we briefly recollect the basic principles of PGSE ${ }^{28-30} \mathrm{NMR}$. The basis for diffusion-sensitive experiment is the application of field gradients pulses (PFG) of duration $\delta$ and increasing intensity along a defined direction (usually the z-axis). The magnetic field gradient indirectly labels the position of NMR-active nuclei introducing a spatial dependence to their Larmor frequency $\omega_{0}$, according to:

$\omega_{t o t}(z)=\omega_{0}-\gamma g(z)$

where $\gamma$ is the gyromagnetic ratio and the first term represents the contribution from the static field $\mathrm{B}_{0}$. The application of a gradient pulse of length $\delta$ and magnitude $g($ i.e., 'area' $=\delta g$ ) creates a position-dependent phase shift defined as follow:

$$
\phi=-\gamma g(z) \delta
$$

and leads to the definition of the reciprocal space vector $q=(\gamma g \delta) / 2 \pi$.

The acquired phase angle depends linearly on both $g$ and duration of the gradient, while $(z)$ the spin position term (in eq. 14) is time-dependent (i.e., $z(t)$ ) due to molecular diffusion during the observation time $t_{d}$. The effect of this phase shift, is not refocused with the application of an opposite gradient pulse (equal in magnitude and duration), consequently the signal intensity decreases. The observed signal attenuation is directly dependent on the space $q$, and time $t_{d}$ variables. Consequently, performing experiments at variable $t_{d}$ time enable to study the motion regime in the micrometers/milliseconds space/time scale.

Usually, in conventional liquid-state probes the gradient coil may produce magnetic gradient pulses only along the $z$ axis, while (x, y, z) gradients are provided in probes particularly designed for magnetic resonance imaging (MRI) applications. In order to properly combine the MAS rotation with PGSE methodologies, modern HR-MAS probes include a gradient coil able to produce a magnetic gradient along the magic angle axis. In this setup, the rotation axis coincide with the magnetic gradients direction so that the signal decay is affected only by molecular motion and uninfluenced by sample spinning. In figure $4 \mathrm{a}$ a graphical representation of the field gradient is shown along with the signal attenuation observed increasing the gradient intensity (panel 4c). A recent instrumental design with two gradient coils on the top and on the bottom of the MAS stator of a Bruker probe reaches about $0.5 \mathrm{~T} / \mathrm{m}$ with a 10 A power supply. Measurement of particularly small molecular displacements would require larger pulsed field gradient intensities $(g \delta)$, limited by the probe hardware performances. The recent advances in PGSE experimental pulse sequences ${ }^{31}$, based on the theory described previously, are all modifications of the Hahn spin echo pulse sequence ${ }^{32}$ (fig. 4b).
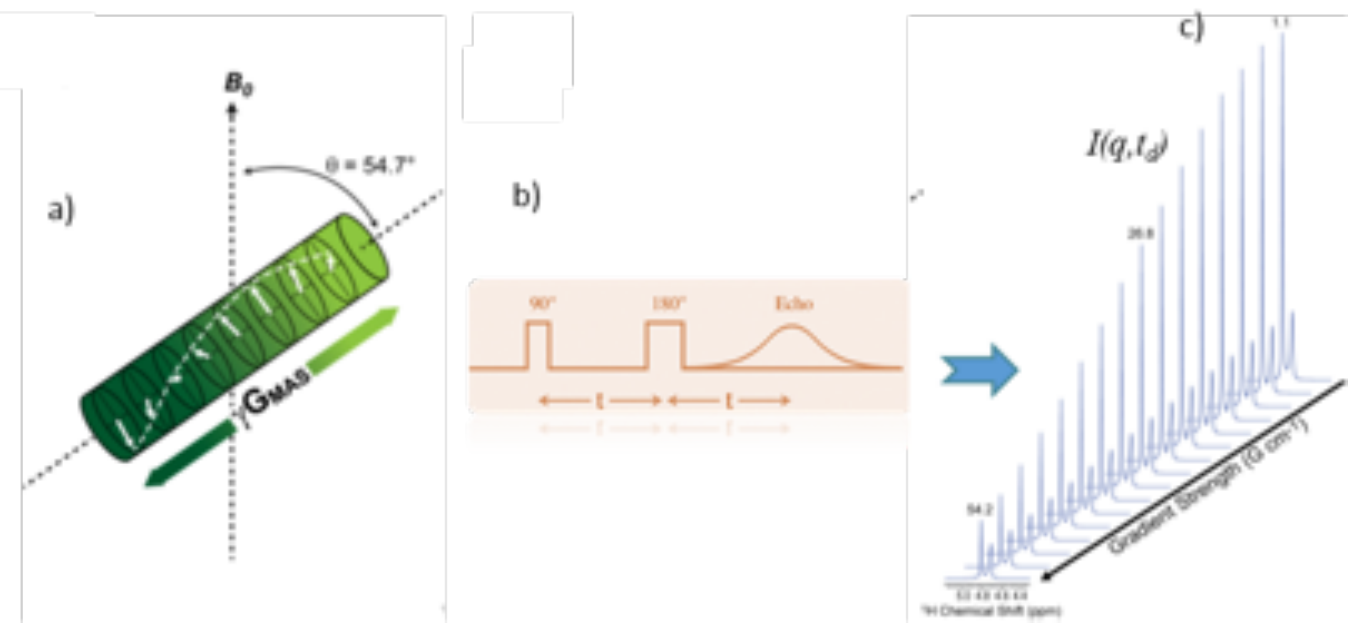
Figure 4. a) Pictorial representation of the gradient produced along the magic angle direction; b) basic spin echo pulse sequence; c) signal decay observed with increasing gradient strength in a conventional PGSE experiment. Source: Adapted from Alam el al. [11].

The attenuation of the NMR signal intensity $I\left(q, t_{d}\right)$, under the influence of molecular motion and the field gradients is given by the relation:

$$
I\left(q, t_{d}\right)=I_{0} \int_{-\infty}^{+\infty} \bar{P}\left(z, t_{d}\right) e^{i q z} d z
$$

Where $\bar{P}\left(z, t_{d}\right)$ is the displacement probability average propagator ${ }^{33,34}$. It denotes the probability density that, after a time $t_{d}$, an arbitrary molecule within the sample is shifted over a distance $\mathrm{z}$ in the direction of the applied field gradient. A Fourier transformation (FT) relates the NMR signal decay with the molecular diffusivity.

\subsection{Translational motion in isotropic systems}

In a homogeneous infinitely extended medium, Fickian diffusion is characterized by a molecular mean square displacement (MSD), $\left\langle z^{2}\right\rangle$, scaling linearly with time:

$$
\left\langle z^{2}\left(t_{d}\right)\right\rangle=n D t_{d}
$$

where $\mathrm{D}$ is the molecular diffusion coefficient (the factor $n$ is 2, 4, or 6 for the cases of one-, two- and three-dimensional motion). In the simplest case, for free and unrestricted diffusion, the mean propagator is given by a Gaussian function that broadens with the increase in the observation time $t_{d}$, and is defined as follows:

$$
\bar{P}\left(z, t_{d}\right)=I_{0} \sqrt{\frac{\pi}{D t}} \exp ^{-\frac{z^{2}}{4 D t}}
$$

The experimental signal intensity $I\left(q, t_{d}\right)$ is related to the variable $\left\langle z^{2}\right\rangle$ at each time $t_{d}$ according to the Stejkal-Tanner equation $^{35,36}$ :

$$
I\left(q, t_{d}\right)=I\left(0, t_{d}\right) e^{-\frac{1}{2} q^{2}\left\langle z^{2}\left(t_{d}\right)\right\rangle}
$$

The numerical value of the molecular diffusion coefficient $D$ can be calculated either by fitting eqs. 18-16, or from the full width at half-maximum:

$\Delta\left(z_{0.5}\right)=2[4 D t \ln 2]^{1 / 2}$

Equations (15-18) describe not only the free diffusion motion of small molecules dissolved in low viscosity liquid samples, but also many diffusion processes occurring in the presence of obstacles or heterogeneities whenever the observation time is much larger than the characteristic time- and length-scales associated with these obstacles ${ }^{37,38}$.

Diffusion motion is closely related to molecular size, as seen from the Stokes-Einstein equation ${ }^{39}$ :

$$
D=\frac{k_{b} T}{6 \pi \eta r_{s}}
$$

where $\mathrm{k}_{\mathrm{b}}$ is the Boltzmann, $\mathrm{T}$ the absolute temperature, $\eta$ the medium viscosity and $\mathrm{r}_{\mathrm{s}}$ the hydrodynamic radius. Equation 20 indicates that, by measuring the diffusion coefficient of a given molecular species in solution, is possible to obtain information on its effective size and, therefore, on specific molecular interactions or aggregation phenomena ${ }^{3,40}$. This type of motion is commonly found in isotropic, liquid-state solutions.

\subsection{Restricted and anisotropic motion}

In complex systems such as heterogeneous materials, the signal attenuation often reflects several diffusion processes, including a combination of free and restricted motion regimes characterised by anisotropic contributions. In such systems, (e.g. gels or polymers) where there are barriers prohibiting free diffusion, an increase in the diffusion time $t_{d}$ does not 
imply an increase in the mean displacement of the diffusing species. In this case, the MSD exhibits a power law relation with the observation time $t_{d}$, according to:

$\left\langle z^{2}(t)\right\rangle=n D^{\prime} t_{d}^{\alpha}$

where $D^{\prime}$ is a generalized diffusion coefficient (whose units are $\alpha$-dependent) and the parameter $\alpha$ is the anomalous diffusion exponent. Its numerical value can be determined as log-log plot of the MSD versus the observation time $t_{d}$. When $0<\alpha<1$ the diffusion process is sub-diffusive, for $\alpha>1$ is super-diffusive and when $\alpha=1$ the diffusion is Gaussian and the relation (16) is recovered. In all these cases, the Stejkal-Tanner equation still holds, but only an apparent diffusion coefficient may be determined by fitting eq. 18.

Heterogeneous rigid porous systems ${ }^{41,42}$ give rise to ordinary $(\alpha=1)$ or sub-diffusive processes $(\alpha<1)$ depending on the dimension of the pores as physical barriers. In soft heterogeneous media, such as gels, the motion of solute molecules may be affected by more complex mechanisms due to drug-polymer interactions resulting in molecular-trapping into geometrically restricted zones. These phenomena are well described in the framework of the continuous time random walk (CTRW) model ${ }^{43,45}$. Anisotropic diffusion may be found in cases where the barriers which impose restriction are not uniformly distributed in the three-dimensional network.

\section{Applications in drug delivery}

In this paragraph we describe two case-studies on the use of ${ }^{1} \mathrm{H}$ HR-MAS NMR spectroscopy to spot on the transport properties of encapsulated active molecules in polymeric matrices of potential use for drug delivery. The cases are labelled according to the type of polymers: i) cyclodestrin nanosponges swollen polymers, ii) agarose-carbomemer co-polymers forming hydrogels.

\subsection{Cyclodextrin nanosponges (CDNS) polymers}

Cyclodextrins are well known macro-cyclic oligosaccharides containing D-glucopyranose units linked via $\alpha(1 \rightarrow 4)$ glycosidic bonds. The usual nomenclature adopts the greek prefixes $\alpha, \beta$ and $\gamma$ for the 6,7 and 8 glucose macroring units in the order, leading to $\alpha, \beta$ - and $\gamma$-cyclodextrin $(\alpha \mathrm{CD}, \beta \mathrm{CD}$ and $\gamma \mathrm{CD})$. The main feature of $\mathrm{CDs}$ is the presence of an hydrophilic external surface, making them water soluble (although with significant different values of solubility among them), and an internal hydrophobic cavity, amenable to host lipophilic molecules by establishing van der Waals interactions and forming stoichiometric, non-covalent inclusion complexes. Such a feature has been extensively exploited to improve water solubility of poorly soluble drugs and/or enhancing the bioavailability of some active pharmaceutical ingredients (API) ${ }^{46}$. However, CDs can be considered also as potential monomeric units for larger architectures. Each glucose unit has indeed three free hydroxyl functional groups available for functionalization, for example via polymerization reaction with suitable multifunctional crosslinkers. A typical example is the reaction of CDs - mainly $\beta C D$ - with activated derivatives of tetracarboxylic acids, such as pyromellitic anhydride (PMA) or ethylenediaminetetraacetic acid dianhydride (EDTAn), or with carbonylating agents equivalent to phosgene, such as diphenilcarbonate (DPC) or carbonyldiimidazole (CDI). The resulting reaction products are generally cross-linked polymers, very often amorphous, characterized by the presence of two types of molecular voids: the cavity of the CD units and the empty spaces generated by the random process of cross-linking. The nanoporous nature of these materials justifies the name of "cyclodextrin nanosponges (CDNS)" commonly used for these derivatives ${ }^{47}$. In many cases it was found that some classes of CDNS can be swollen with aqueous solutions of API, thus allowing the preparation of drugloaded hydrogels. The first HR-MAS NMR characterization of a small molecule mimicking a drug inside a CDNS hydrogel was presented in $2011^{48}$. The high resolution spectra of sodium fluoresceine could be obtained under HR-MAS NMR consitions, thus opening the route to the exploitation of the large repertoire of 1D- and 2D NMR experiments to 
directly monitor the molecular state and the dynamics of the confined drug. A systematic application of the diffusion measurements of a drug of interest (ibuprofen sodium salt, IP) in hydrogels prepared from $\beta C D$ and EDTAn was first published in $2014^{49}$. Since the properties of the CDNS can be modulated by varying the molar ratio $\mathrm{n}$ of crosslinker to $\mathrm{CD}$, two different formulations of the nanosponges CDNDEDTA were tested with $\mathrm{n}=4$ and $\mathrm{n}=8$, referred to as CDNSEDTA 1:4 and CDNSEDTA 1:8, respectively. The viscous, drug loaded gel was then loaded in the HR MAS rotors in order to carry out the diffusion measurements by the PGSE experimental set up. The main purpose of the experiments was to work out the type of motion the drug undergoes inside the hydrogel. This type of information can be conveniently exploited in the rational design of a molecular scaffold for drug delivery with known release properties. It is important to stress here that the goal of the experiment is not the formal determination of the apparent diffusion coefficient $\mathrm{D}$, rather is the determination of the mean squared displacement in the selected time window $t_{d}$. This approach, based on "variable diffusion time" experiments, ends up with a collection of experimentally determined $\left\langle z^{2}\left(t_{d}\right)\right\rangle$ values for any $t_{d}$ used in the arrayed experiments. The determination of the molecular MSD is the key passage to get a first indication of the diffusion regime the drug undergoes in the polymer matrix.

A flow chart of the experimental set-up - commonly referred to as the gradient-dependent echo decay analysis (GDES) - can described in the following two steps:

i) The first step consists of the acquisition of a collection of PGSE decay curves as a function of the diffusion delay $t_{d}$. The linearized form of equation 18 (eq. 22) allows one to extract the collection of the experimental mean squared displacements experienced by the diffusing drug in the observe interval between the minimum and the maximum $t_{d}$. In this case study the $t_{d}$ values were in the interval $50-170 \mathrm{~ms}$.

$$
\ln \frac{I\left(q, t_{d}\right)}{I\left(0, t_{d}\right)}=-\frac{1}{2} q^{2}\left\langle z^{2}\left(t_{d}\right)\right\rangle
$$

ii) The second step is based on the relationship between $\left\langle z^{2}\left(t_{d}\right)\right\rangle$ and $t_{d}$, as described in the generalized way by eq. 21 . From the experimental standpoint, the exponent of the power law relating the MSD and diffusion time can be determined by a linear regression of a simple log-log plot. Figure 5 shows the results obtained during the investigation on the transport of IP in CDNSEDTA 1:4 and 1:8.

(a)

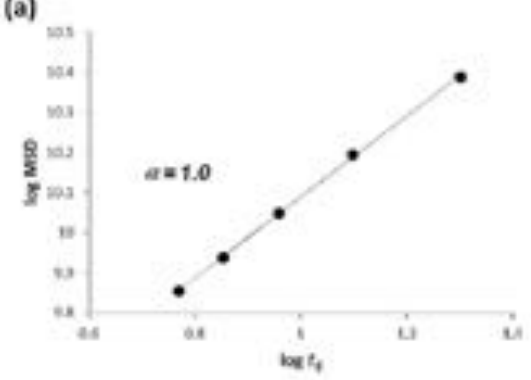

(b)

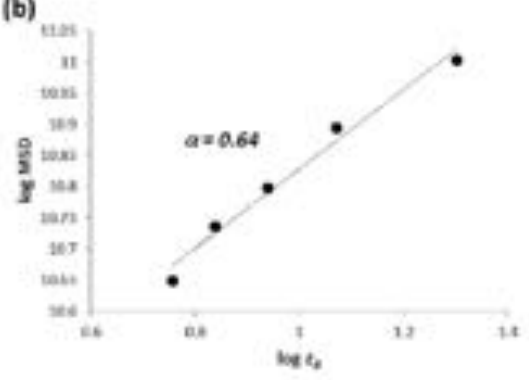

(c)

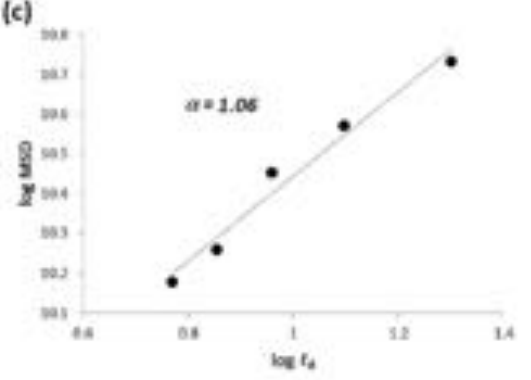

Figure 5. a) logarithmic regression plot of MSD $v s$ diffusion time of IP in $\mathrm{D}_{2} \mathrm{O}$ solution. This is a "control" experiment carried out with a conventional NMR probe for liquids. The slope of the linear plot corresponds to $\alpha=1$ and it is a paradigmatic example of purely Fickian diffusion, with MSD scaling linearly with the diffusion time; b) experimental 
result of the multiple diffusion time experiment on IP confined in a hydrogel of CDNSEDTA 1:4. The obtained slope is indicative of a marked subdiffusive behaviour; c) same as (b) but with IP entrapped in CDNSEDTA 1:8. The $\alpha$ value reported in the plot is slightly greater than 1, thus suggesting a superdiffusive motional regime (see text for caveat). Source: Ferro et al. 2014 [49]. Reprinted with permission

The picture emerging from Figure 5 is that of a modulation of the type of motion of the same molecule by acting on the surrounding lattice. The starting point is the water solution, where no anomalous diffusivity is detected, pointing towards a Fickian transport regime. This experiment outlines the reference state, where no effects of interactions of the solute with the polymer backbone are present, nor any effect of restricted diffusion in a confined empty pore. Plot b) of Fig. 5 shows clearly the first important finding: the IP molecules undergo a transition from normal Fickian diffusion $\left(\mathrm{D}_{2} \mathrm{O}\right.$ solution) to a subdiffusive behaviour when encapsulated in hydrogel of CDNSEDTA 1:4. This is highlighted by the experimental value $\alpha=0.64$. A second important point coming out from the comparison of plots b) and c) of Fig. 5 is that the confinement of IP in CDNSEDTA obtained with different preparations - molar ratios of CD to crosslinker 1:4 and 1:8, respectively - leads to different effects on the diffusive regime: the diffusivity of IP is significantly influenced by the polymer preparation. In the present case, the $\alpha$ exponent passes from 0.64 of the 1:4 preparation to the value 1.06 of the 1:8. The value $\alpha=1.06$ indicates a transport behaviour on the border between normal diffusion and a slightly superdiffusive regime. The main conclusion is that the transport properties of IP can be modulated by the polymer preparation keeping constant other physico-chemical parameters, such as temperature and drug concentration. This is actually an important indication for the rational design of drug delivery and release systems. A clear-cut rational of this behaviour is not yet available. Suffices it to mention, at this stage, that significant variation in both the void size (meshsize) of CDNS and the sensitivity of the nanosponges to hydration in terms of swelling kinetics were observed by small angle neutron scattering (SANS) experiments ${ }^{50}$. However, the prediction of the type of motion on the basis of the pure void size of the crosslinked polymer is an oversimplified approach leading to non-consistent conclusion, and great care should be take when trying to correlate the diffusive regime to the pure geometrical descriptors of the scaffold. A tentative explanation of the transition from subdiffusive to normal/superdiffusive motion in the cases of Fig. 5 should be based on extra factors, including the larger presence of negatively charged $\mathrm{COO}^{-}$dangling groups in CDNSEDTA 1:8 with respect to CDNSEDTA 1:8. This is a consequence of the fact that increasing the molar ratio CD/EDTAn leads to increasing crosslinking of the resulting polymer up to $1: 6$, then further increase in the molar ratio results branching rather than further reticulation, basically for steric reasons ${ }^{51}$. A simple sketch is reported in Figure 6.

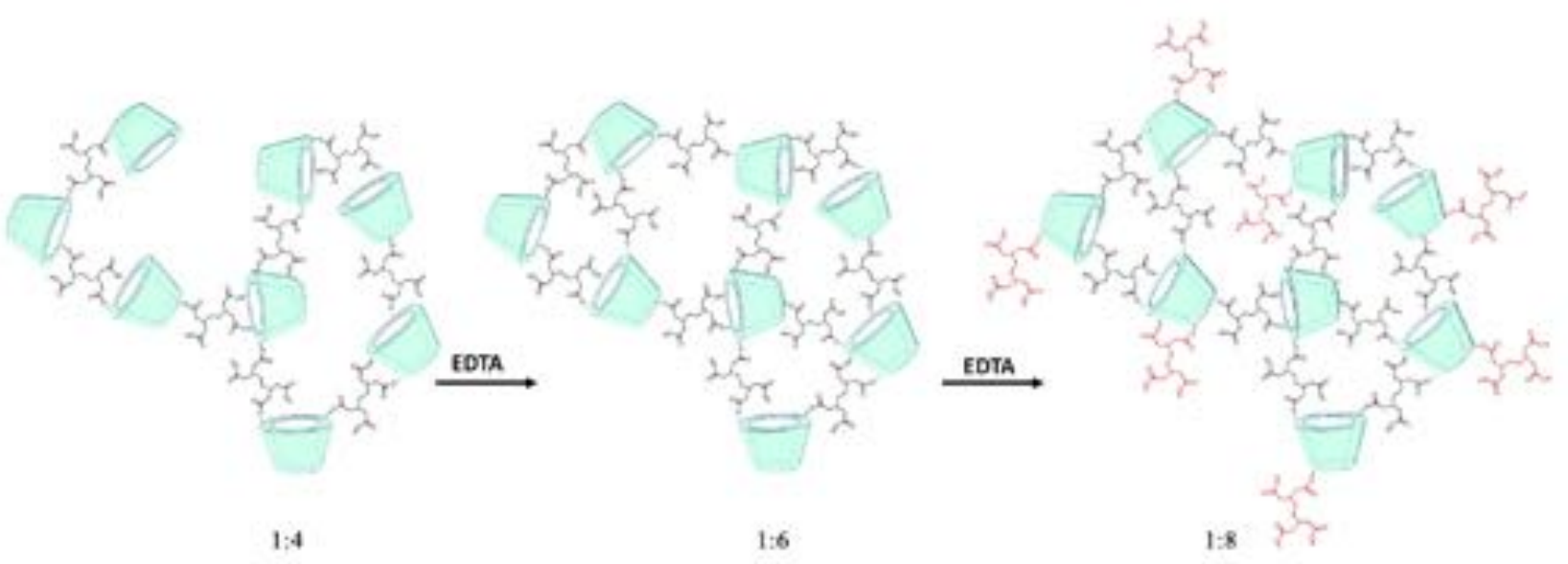

Figure 6. Sketch of the formation of CDNSEDTA. Left: at low excess of EDTEn the crosslinking is the dominant process. Middle: the 1:6 molar ratio was found to be the cross-over value for competitive processes of crosslinking and branching. 
Right: the sketch highlights the presence of the dangling carboxylic groups resulting from hydrolysis of EDTA dianhydride after the first condensation reaction with the $\mathrm{OH}$ groups of the growing cyclodextrin polymer. Source: Ferro et al. 2014 [49]. Reprinted with permission

From the standpoint of the transport phenomena inside the hydrogels of CDNSEDTA 1:8, the presence of negatively charged carboxylate groups provides the pore surface of the nanosponge voids with a negative electric potential which, in turn, may be responsible of the acceleration effects. The diffusion experiments on CDNSEDTA 1:8 were carried out at $\mathrm{pH}$ values in the range 6.5-6.9. Considering the $\mathrm{pKa}$ values of EDTA reported in the literature, the ionization state of the dangling residues of EDTA in the nanosponge are expected to provide the overall negative electric potential able to attenuate, or to overwhelm, the subdiffusive behaviour detected in the 1:4 formulation.

Finally, this case also propose a methodological conclusion: ${ }^{1} \mathrm{H}$ HRMAS setup combined with PFG NMR spectroscopy is a unique physical method able to monitor the diffusivity of API in the realistic molecular environment for delivery, targeting or controlled release.

\subsection{Agarose-carbomer co-polymers hydrogels.}

In this section we present a case study based on API entrapped in a class of polymeric hydrogels designed for drug delivery and tissue engineering and based on Carbomer 974P and Agarose. The detailed description of the preparation of hydrogels and loading with API is reported in ref. 52. The synthetic procedure allows a good control of the porosity of the resulting polymer, thus offering the route to a variable and controlled mesh-size family of scaffolds for molecular encapsulation. The main purpose of this section is to illustrate how the diffusivity data obtained via ${ }^{1} \mathrm{H}$ HR-MAS NMR spectroscopy can be exploited to derive a generalized model of the drug transport accounting for both drug-polymer and drug-drug interactions.

The former interactions were investigated by monitoring the diffusivity of ethosuximide (ESM), a drug classified as anticonvulsant and used for the treatment of epilepsy, inside hydrogel formulations based on swollen co-polymers obtained from agarose and carbomer $(\mathrm{AC})^{53}$. The diffusion coefficients of ESM in standard $\mathrm{D}_{2} \mathrm{O}$ solutions were determined and compared to the homologous data in AC hydrogel measured via ${ }^{1} \mathrm{H}$ HR-MAS NMR methods. Figure 7 shows the molecular formula, atom numbering and the proton spectrum of ESM in AC hydrogel at $75 \mathrm{mg} \mathrm{mL}^{-1}$ concentration.

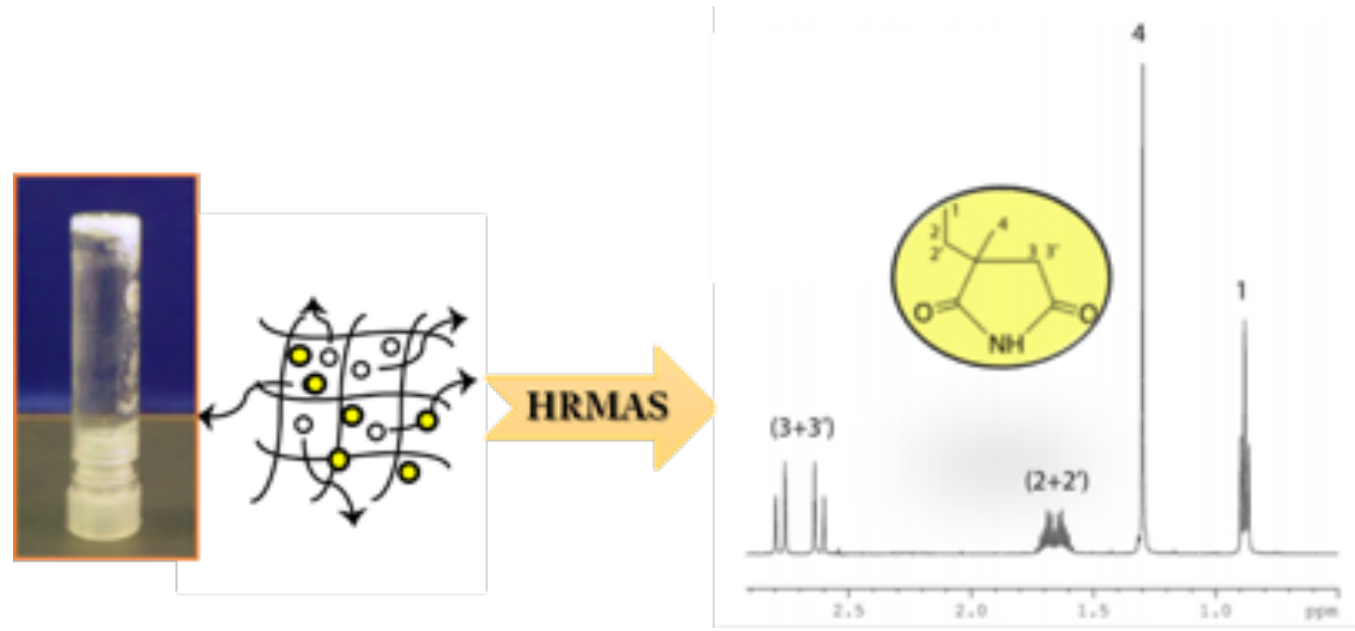

Figure 7. Sketch of the experimental methodology. ${ }^{1} \mathrm{H}$ HR-MAS NMR spectrum of ESM in AC hydrogels with peak assignment. Source: Adapted from Rossi et al. 2015 [53].

The comparison of the diffusion coefficients measured in $\mathrm{D}_{2} \mathrm{O}$ and in $\mathrm{AC}$ hydrogels allowed to spot on possible differenced of the transport properties in the two different environments. The results are shown in Table 1. 
Table 1. Diffusion coefficients of ESM as a function of concentration measured via PGSE NMR in $\mathrm{D}_{2} \mathrm{O}$ solution $\left(\mathrm{D}_{0}\right)$, in AC hydrogel by HR-MAS NMR (D) and their ratio (D/D $)$. [Reprinted from ref. 53 with permission]

\begin{tabular}{llll}
\hline $\begin{array}{l}\text { ESM concentration } \\
{\left[\mathrm{mg} \mathrm{mL}^{-1}\right]}\end{array}$ & $\begin{array}{l}\mathrm{Da} \\
\left.\mathrm{m}^{2} \mathrm{~s}^{-1}\right]\end{array}$ & $\begin{array}{l}D_{0}{ }^{[\text {a] }]} \\
{\left[\mathrm{m}^{2} \mathrm{~s}^{-1}\right]}\end{array}$ & $D / D_{0}$ \\
\hline 4 & $0.49 \pm 0.03$ & $0.92 \pm 0.05$ & $0.53 \pm 0.05$ \\
19 & $0.87 \pm 0.05$ & $0.88 \pm 0.05$ & $0.99 \pm 0.08$ \\
38 & $0.89 \pm 0.05$ & $0.86 \pm 0.05$ & $1.03 \pm 0.08$ \\
56 & $0.91 \pm 0.06$ & $0.82 \pm 0.05$ & $1.11 \pm 0.1$ \\
75 & $0.87 \pm 0.05$ & $0.78 \pm 0.05$ & $1.11 \pm 0.12$ \\
\hline
\end{tabular}

[a] All values have to be multiplied by $10^{-9}$.

The reported values indicate that the observed diffusion coefficient of ESM in deuterated water decreased with increasing concentration due to ESM aggregation phenomena affecting both the hydrodynamic radius and the solution viscosity. Surprisingly, the measured diffusion coefficients in AC hydrogels show the opposite trend, with the counterintuitive increase of diffusivity with increasing ESM concentration. This first finding indicates that the molecular environment experienced by ESM in AC dramatically affects the drug diffusivity. A simple model accounting for this behaviour can be designed starting from the following assumptions: i) ESM molecule do interact with the polymer backbone within the hydrogel pores. The main mechanism is adsorption. ii) The adsorption phenomena are important especially at low ESM concentration. The data of Table 1 show that the diffusivity in $\mathrm{AC}$ is lower than in water. iii) At larger concentrations, the adsorption sites are progressively saturated. This causes the ESM molecules to faster, with values of D comparable to those in water at the same concentration. It is important to stress that, at molecular level, the mesh size of the hydrogel is much larger than the mean hydrodynamic radius of ESM which, in turn, experiences a bulk-water like environment. A sketch of this model is reported in Figure 8a.

The assumptions described above can be translated into a mathematical model by combining the Langmuir equation with the Fick's laws. The final equation is reported as:

$\frac{D}{D_{0}}=\frac{\varepsilon}{\epsilon+(1-\varepsilon) \frac{q^{\infty} K}{\left(1-K C_{G}\right)^{2}}}$

Where $\varepsilon$ is the hydrogel porosity porosity ${ }^{54}$, q $\infty$ is the maximum adsorbed ESM, $\mathrm{K}$ is the Langmuir adsorption parameter, $\mathrm{CG}$ is the drug concentration in the gel. Equation 23 was validated against the experimental diffusivities measured via HR-MAS NMR. The fitting is reported in Figure $8 \mathrm{~b}$. The experimental trend is very well reproduced by eq. 23 . The low concentration part of the plot is of particular importance for drug delivery since the concentration range where the adsorption phenomena are dominant coincides with the drug concentrations used in clinical trials.
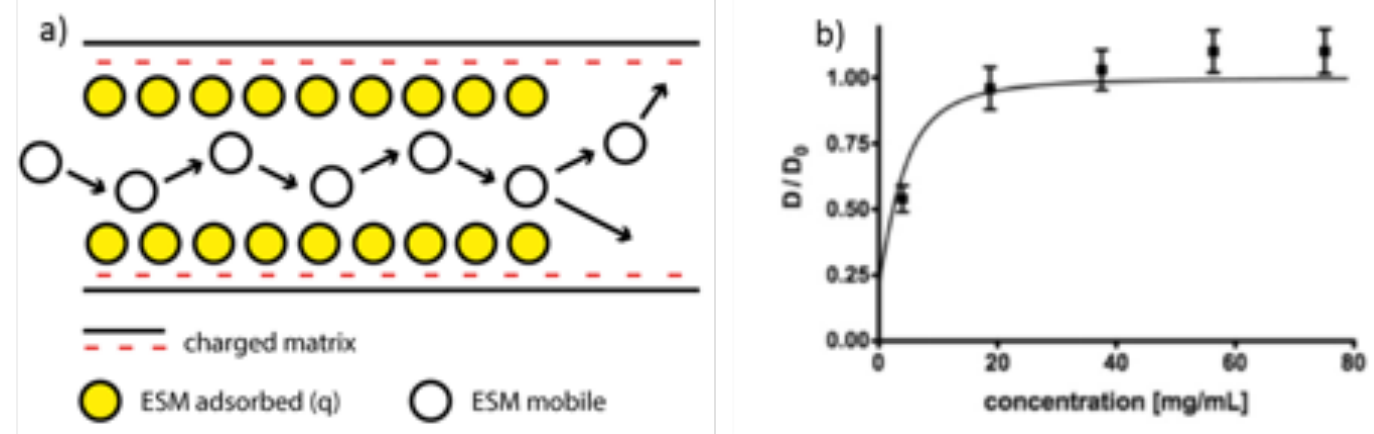

Figure 8. a) Simple scheme of the adsorption-partitioning model; b) Experimental trend of $D / D_{0}$ for ESM at variable concentration (squares) and predicted (line) by equation 23. Source: Rossi et al. 2015 [53]. Reprinted with permission 
The second aspect - drug-drug interactions - was also considered within the same type of hydrogel. ${ }^{55}$ The observed molecule was sodium fluorescein (SF). SF is not an active pharmaceutical ingredient, however it mimics very well steric hindrance, polarity and aggregation phenomena of some important drugs such as corticosteroids and anti-inflammatory drugs (methylprednisolone, ibuprofen, estradiol, etc.).

Figure 9 shows the ${ }^{1} \mathrm{H}$ HR-MAS NMR spectra of SF in AC hydrogels at increasing concentrations. The spectra show selective chemical shift dependence on SF concentrations, diagnostic of intermolecular interactions, possibly $\pi-\pi$ stacking. On the other side, the narrow linewidth of the NMR signals even at large concentrations clearly rules out the formation of large aggregates, rather suggesting the formation of oligomers (dimers, trimers). The diffusion coefficients of SF in deuterated water and in AC hydrogels were measured. The results are summarized in Table 2.

Table 2. Diffusion coefficients obtained via ${ }^{1} \mathrm{H}$ NMR of SF in water and in AC hydrogels as a function of SF concentration. The ratios are referred to $D$ at infinite dilution. [Reprinted from ref. 53 with permission]

\begin{tabular}{|c|c|c|c|c|}
\hline $\begin{array}{l}\text { SF concentration } \\
{\left[\mathrm{mg} \mathrm{mL}^{-1}\right]}\end{array}$ & $\begin{array}{l}D_{\mathrm{od}}^{|\mathrm{a}|} \\
{\left[\mathrm{m}^{2} \mathrm{~s}^{-1}\right]}\end{array}$ & $\begin{array}{l}D_{\text {wane }} \\
{\left[\mathrm{m}^{2} \mathrm{~s}^{-1}\right]}\end{array}$ & $D_{\text {oed }} / D_{\text {ht }}$ & $D_{\text {waned }} / D_{i t}$ \\
\hline 10 & $5.4 \pm 0.5$ & $4.5 \pm 0.4$ & $0.98 \pm 0.06$ & $0.82 \pm 0.08$ \\
\hline 50 & $4.7 \pm 0.4$ & $2.9 \pm 0.3$ & $0.85 \pm 0.07$ & $0.53 \pm 0.06$ \\
\hline 100 & $4.2 \pm 0.5$ & $2.9 \pm 0.3$ & $0.76 \pm 0.05$ & $0.53 \pm 0.05$ \\
\hline 150 & $3.3 \pm 0.3$ & $2.4 \pm 0.3$ & $0.61 \pm 0.06$ & $0.44 \pm 0.05$ \\
\hline 200 & $3.9 \pm 0.4$ & $2.9 \pm 0.3$ & $0.70 \pm 0.07$ & $0.53 \pm 0.05$ \\
\hline
\end{tabular}

[a] All values have to be multiplied by $10^{-10}$.

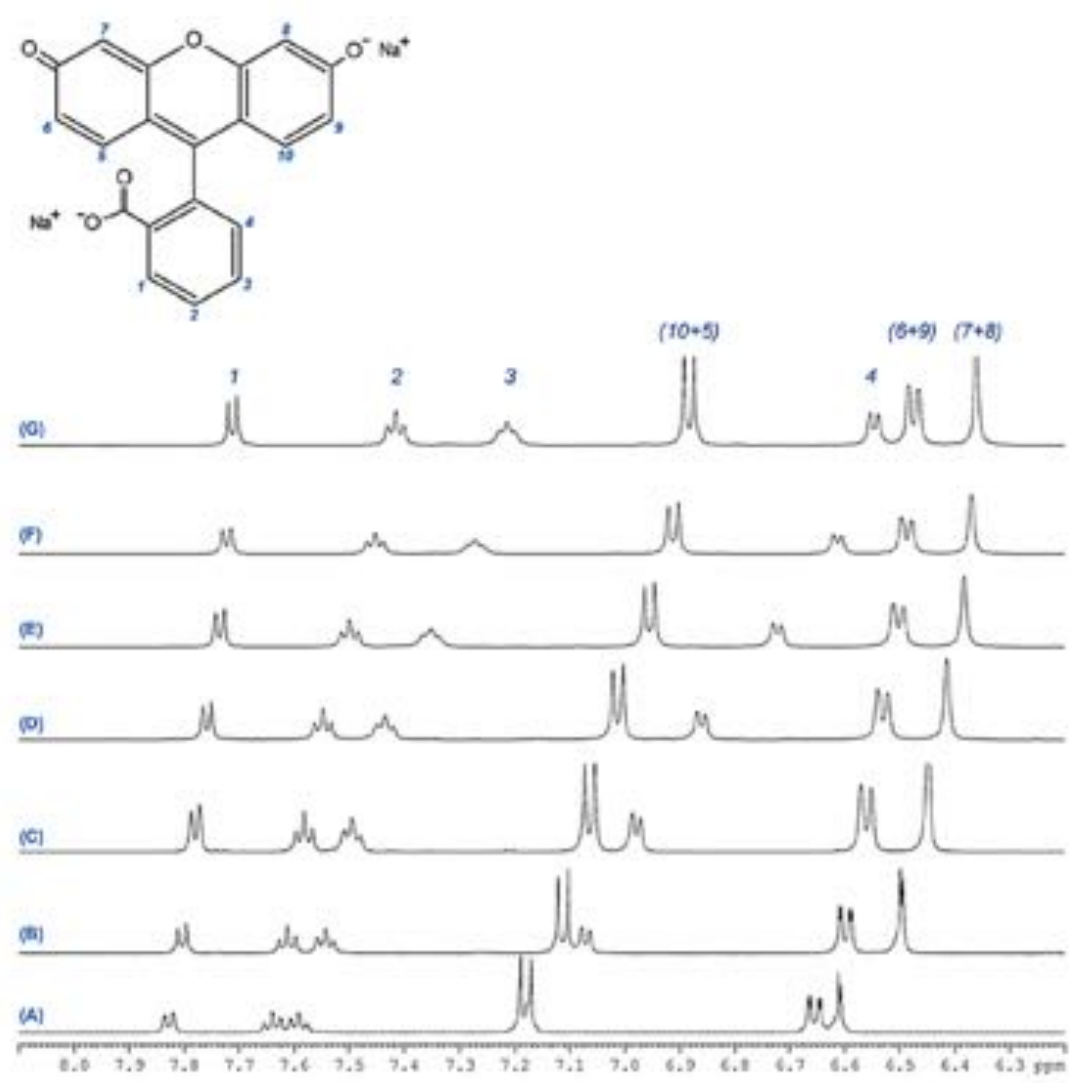

Figure 9. ${ }^{1} \mathrm{H}$ HR-MAS spectra of SF in AC at the following concentrations: A) 6, B) 12.5, C) 25, D) 50, E) 100, F) 150, and G) $200 \mathrm{mgmL}^{-1}$. Molecular formula and atom numbering of SF are also shown. Source: Rossi et al. 2016 [55]. Reprinted with permission

The values of Table 2 show the decrease of $D$ with increasing concentration in both water and hydrogel, as expected, but also show the counterintuitive finding that SF diffusion coefficients $D$ in gel are always larger than in water at the same 
concentration. A suitable model accounting for these experimental aspects can be formulated assuming the presence, both in water and in the hydrogel, of monomeric, dimeric and trimeric species in equilibrium. The observed diffusion constant by NMR can be thus expressed as:

$D=\frac{C_{M}}{C_{\text {tot }}} D_{M}+\frac{C_{D}}{C_{\text {tot }}} D_{D}+\frac{C_{T}}{C_{\text {tot }}} D_{T}$

Where $C_{i}$ and $D_{i}$ are the concentrations and the diffusion coefficients of the monomer (M), dimer (D) and trimer (T). Using similar arguments as in the previous section, a suitable mathematical model can be built on following hypotheses: i) only monomeric SF can be adsorbed onto the polymer surface. The process of adsorption hampers the aggregation of SF reducing the monomers available for the formation of dimers and trimers. This effect if particularly important at low DF concentration. ii) At higher SF concentration, the adsorption sites are progressively saturated and SF can diffuse faster, showing a behaviour like in bulk water. Considering that the hydrodynamic radius id much smaller than the mesh size of the pore where the solute is confined, the SF molecules can diffuse with a high free motion. Also in this case, the adsorption phenomena are expected to play a dominant role at low SF concentrations and negligible at high concentrations. A graphical sketch of the model is shown in Figure 10.

As in the previous case, a mathematical model combining the adsorption of SF, the speciation of SF and the Fick's law leads to a general expression of the observed $D$. (Equation 25)

$D_{g e l}=\frac{\varepsilon}{\epsilon+(1-\varepsilon) \frac{q^{\infty} K}{\left(1-K C_{G}\right)^{2}}}\left(\frac{C_{M}}{C_{t o t}} D_{M}+\frac{C_{D}}{C_{t o t}} D_{D}+\frac{C_{T}}{C_{t o t}} D_{T}\right)$

Equation 25 reasonably predicts the observed trend of the NMR determined D values as a function of SF concentration, as shown by Figure 10 .
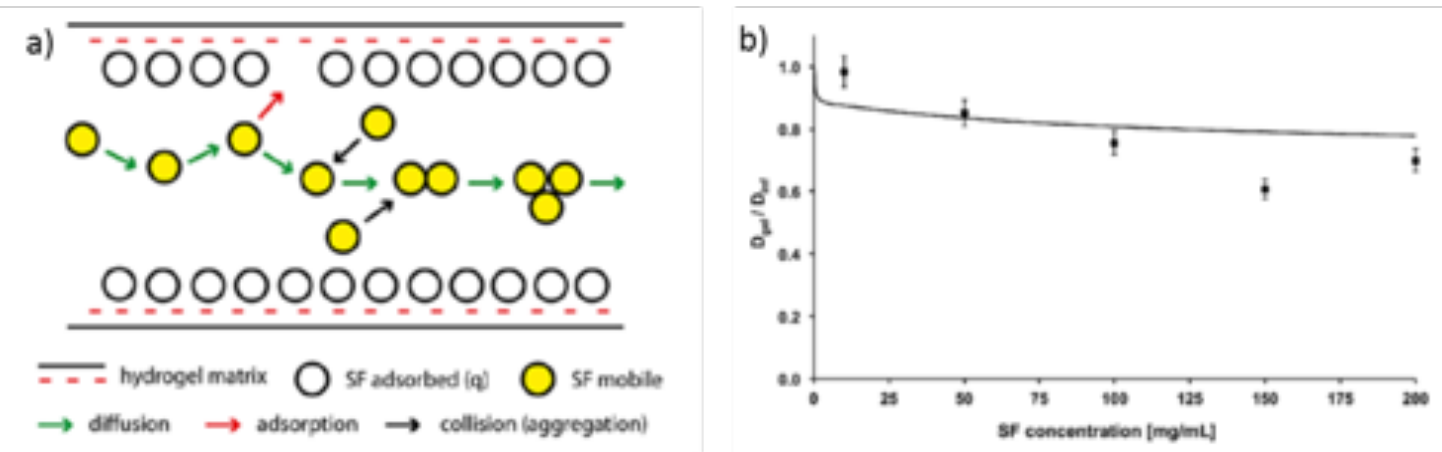

Figure 10. a) Scheme of the partition model. The white circles indicate the monomeric SF adsorbed onto the polymeric surface, the yellow circles SF diffusing and colliding in solution within the hydrogel pores. The adsorption, diffusion and collision acts are sketched by the green, red and black arrows in the order; b) Experimental (squares) and predicted (line) trend of normalized diffusivity of SF as a function of concentration in AC hydrogels. Source: Rossi et al. 2016 [55]. Reprinted with permission

In conclusion, the dominant adsorption phenomena at low SF concentration inhibit drug association leading to the nonintuitive diffusivity of SF faster than in pure water, where the dimer and trimer formation is present even at low concentration. From the methodological standpoint, the availability of diffusion data by direct observation of SF transport inside the hydrogel by HR-MAS NMR investigation is a plus in formulating realistic and quantitative models of diffusion in confined media.

\section{Final remarks}

High Resolution-MAS is a relatively recent NMR technique developed to allow the direct investigation of semi-solid/soft materials, using low spinning speed, for several field of application. When combined with PFG methods, HR-MAS-PFG 
represents a simple and accurate tool to obtain detailed insights on the local transport processes in the hydrogels/polymer formulations especially designed for controlled drug release.

In heterogeneous soft materials, the dynamic processes may be ascribed to complex phenomena due to the different chemical/physical interactions between all the components of the multicomponent systems in gel phase. In particular, drug-drug aggregation phenomena, drug-polymer chemical interactions, or polymer matrix as physical barrier to drug motion need to be considered jointly. Accordingly, the NMR experimental data are analysed using mathematical models specially formulated for considering all these phenomena.

HR-MAS PFG NMR methods together with appropriate mathematical models opens the possibility to analyse the molecular dynamic regime in a time range from few to hundreds of milliseconds simultaneously gaining information about the structure-dependent diffusional behaviour. A thorough understanding/prediction of the drug transport behaviour in hydrogel/polymer scaffolds is crucial to establish an accurate connection between the dynamics at molecular level and macroscopic drug release kinetics in novel materials designed for controlled drug delivery. Future work will combine theory and experiments to address this connection as done in our previous work ${ }^{56}$ on ibuprofen loaded in AC hydrogels.

\section{References}

[1] A. C. F., Ribeiro, M. A., Esteso, Transport properties for pharmaceutical controlled-release systems: A brief review of the importance of their study in biological systems, Biomolecules 8 (2018) 178.

[2] M. D., Mantle, NMR and MRI studies of drug delivery systems, Current Opinion in Colloid and Interface Science 18 (2013) 214.

[3] E. Okamura, Solution NMR to quantify mobility in membranes: Diffusion, protrusion, and drug transport processes, Chemical and Pharmaceutical Bulletin 67 (2019) 308.

[4] L. O., Sillerud, R. S. Larson, Advances in nuclear magnetic resonance for drug discovery, Bioinformatics and Drug Discovery 910 (2012) 910195.

[5] J.D., Figueroa-Villar, L.W., Tinoco, Spin-lattice relaxation time in drug discovery and design, Current Topics in Medicinal Chemistry 9 (2009) 811.

[6] A.L., Skinner, J.S., Laurence, High-field solution NMR spectroscopy as a tool for assessing protein interactions with small molecule ligands, J. of Pharmaceutical Science 97 (2008) 4670.

[7] W. E., Maas, F. H., Laukien, D. G., Cory, Gradient, high resolution, magic angle sample spinning NMR J. Am. Chem. Soc. 118 (1996) 13085.

[8] W. P. Power, High Resolution Magic Angle Spinning - Applications to Solid Phase Synthetic Systems and Other Semi-Solids, Annu. Rep. NMR Spectrosc. 51 (2003) 261.

[9] J. E., Jenkins, M. R., Hibbs, T. M., Alam, Identification of multiple diffusion rates in mixed solvent anion exchange membranes using high resolution MAS NMR, ACS Macro Letters, 1 (2012) 910.

[10] S., Iqbal, F., Rodrıguez-LLansola, B., Escuder, J. F., Miravet, I., Verbruggen and R., Willem, HRMAS ${ }^{1} \mathrm{H}$ NMR as a tool for the study of supramolecular gels, Soft Matter 6 (2010) 1875.

[11] T. M., Alam and J. E., Jenkins, HR-MAS NMR Spectroscopy in Material Science, in Advanced Aspects of Spectroscopy, ed. M. A.Farrukh, InTech, 10, (2012) 279.

[12] B., Sitter, T. F., Bathen, M. B., Tessem, and I. S., Gribbestad, High-resolution magic angle spinning (HR MAS) MR spectroscopy in metabolic characterization of human cancer, Prog. Nucl. Magn. Reson. Spectrosc., 54 (2009) 239.

[13] W., Li, Multidimensional HRMAS NMR: a platform for in vivo studies using intact bacterial cells, Analyst 131 (2006) 777.

[14] J. C., Lindon, O. P., Beckonert, E., Holmes, J. K., Nicholson, High-resolution magic angle spinning NMR spectroscopy: Application to biomedical studies 55 (2009) Prog. Nucl. Magn. Reson. Spectrosc. 79.

[15] M. J., Duer, Introduction to solid-state NMR spectroscopy, Blackwell Publishing Ltd., Oxford (2004).

[16] D. D., Laws, H.-M. L., Bitter, and A., Jerschow, Solid-State NMR Spectroscopic Methods in Chemistry, Angew. Chem. Int. Ed. 4120023096.

[17] C. P., Slichter, Principles of Magnetic Resonance 1990 (Berlin: Springer).

[18] M. H., Levitt, Spin Dynamics: Basics of Nuclear Magnetic Resonance Wiley.

[19] K., Schmidt-Rohr, W., Spiess, Multidimensional solid-state NMR and polymers (1994) Academic Press Ltd., London.

[20] E. R., Andrew, A., Bradbury, R. G., Eades, Nuclear magnetic resonance spectra from a crystal rotated at high speed Nature 182 (1958) 1659.

[21] I. J., Lowe, Free induction decays of rotating solids Phys Rev Let 2 (1959) 285-287. 
[22] F. D., Doty, G., Entzminger and Y. A., Yang, Magnetism in high-resolution NMR probe design. II: HR MAS, Concepts Magn. Reson. 10 (1998) 239.

[23] A., Sodickson, and D. G. Cory, Shimming a High-Resolution MAS Probe, J. Magn. Reson. 128 (1997) 87.

[24] W. E., Maas, A., Bielecki, M., Ziliox, F. H., Laukien, D. G., Cory, Magnetic Field Gradients in Solid State Magic Angle Spinning NMR, J. Magn. Reson. 141 (1999) 29.

[25] W. E., Maas, F. H., Laukien, D. G., Cory, Gradient, High Resolution, Magic Angle Sample Spinning NMR, J. Am. Chem. Soc. 118 (1996) 13085.

[26] S., Viel, F., Ziarelli, G., Pages, C., Carrara, S., Caldarelli, Pulsed field gradient magic angle spinning NMR selfdiffusion measurements in liquids, J. of Magn. Reson. 190 (2008) 113.

[27] F., Rossi, R., Ferrari, F., Castiglione, A., Mele, G., Perale and D., Moscatelli, Polymer hydrogel functionalized with biodegradable nanoparticles as composite system for controlled drug delivery, Nanotechnology 26 (2015) 015602.

[28] W. S., Price, Studies of Translational Motion Cambridge University Press: (2009) Cambridge, UK.

[29] M., Zubkov, M. Gary, R. Dennis, T. Stait-Gardner, A. M. Torres, S. A. Willis, G. Zheng and W. S. Price Physical characterization using diffusion NMR spectroscopy Magn. Reson. Chem. 55 (2017) 414.

[30] Callaghan, P.T. Principles of NMR Microscopy; Clarendon Press: Oxford, UK, 1991.

[31] D., Wu, A., Chen, C. S., Johnson, An improved diffusion ordered spectroscopy experiment incorporating bipolar gradient pulses, J. Magn. Reson. Series A 115 (1995) 260.

[32] E. L., Hahn, Spin echoes, Phys. Rev. 80 (1950) 580.

[33] D. G., Cory, A. N., Garroway, Measurement of translational displacement probabilities by NMR: An indicator of compartmentation, Magn. Reson. Med. 14 (1990) 435.

[34] Y., Cohen, Y., Assaf, High b-value q-space analysed diffusion-weighted MRS and MRI in neuronal tissues - a technical review, NMR Biomed. 15 (2002) 516.

[35] D., Sinnaeve, The Stejskal-Tanner Equation Generalized for Any Gradient Shape-An Overview of Most Pulse Sequences Measuring Free Diffusion, Concepts Magn. Reson., Part A, 40 (2012) 39.

[36] P. W. Kuchel, G. Pagès, K. Nagashima, S. Velan, V. Vijayaragavan, V. Nagarajan and K. H. Chuang, Stejskaltanner equation derived in full, Concepts Magn. Reson., Part A 40 (2012) 205.

[37] B., Wang, J., Kuo, S. C., Bae, and S., Granick, When Brownian Diffusion is not Gaussian, Nature Mat. 11 (2012) 481.

[38] B., Wang, S. M., Anthony, S. C., Bae, and S., Granick, Anomalous yet Brownian, Proc. Natl. Acad. Sci., 106 (2009) 15160.

[39] A., Einstein, A new determination of molecular dimensions, Ann. Phys. 19 (1906) 289.

[40] Y., Cohen, L., Avram, L., Frish, Diffusion NMR spectroscopy in supramolecular and combinatorial chemistry: An old parameter - New insights, Angew. Chem. 44 (2005) 520.

[41] A., Cortis, Y., Chen, H., Scher, \& B., Berkowitz, Quantitative characterization of pore-scale disorder effects on transport in "homogeneous" granular media. Phys. Rev. E. 70 (2004) 041108.

[42] J. Karger, D. M. Ruthven, Diffusion in Zeolites and Other Microporous Solids, Wiley, New York.

[43] R., Metzler, J., Klafter, The random walk's guide to anomalous diffusion: a fractional dynamics approach, Physics Reports 339 (2000) 1.

[44] H., Scher and M., Lax, Stochastic Transport in a Disordered Solid. I. Theory, Phys. Rev. B 7 (1973) 4491.

[45] J., Klafter and R., Silbey, Derivation of the Continuous-Time Random-Walk Equation, Phys. Rev. Lett. 44 (1980) 55.

[46] K. H., Frömming, J., Szejtli, Cyclodextrins in Pharmacy, Springer Netherlands (1994).

[47] F., Trotta, and A. Mele, (Eds) Nanosponges: Synthesis and Applications, Wiley, (2019).

[48] A. Mele, F., Castiglione, L., Malpezzi, F., Ganazzoli, G., Raffaini, F., Trotta, B., Rossi, A., Fontana, G., Giunchi, HR MAS NMR, powder XRD and Raman spectroscopy study of inclusion phenomena in $\beta C D$ nanosponges, J. Incl. Phenom. Macrocycl. Chem. 69 (2011) 403.

[49] M., Ferro, F., Castiglione, C., Punta, L., Melone, W., Panzeri, B., Rossi, F., trotta and A., Mele, Anomalous diffusion of Ibuprofen in cyclodextrin nanosponge hydrogels: an HRMAS NMR study, Beilstein J. Org. Chem. 10 (2014) 2715.

[50] B., Rossi, A., Paciaroni, V., Venuti, G. C., Fadda, L., Melone, C., Punta, V., Crupi, D., Majolino, A., Mele, SANS investigation of water adsorption in tunable cyclodextrin-based polymeric hydrogels, Phys. Chem. Chem. Phys. 19 (2017) 6022.

[51] V., Crupi, A., Fontana, M., Giarola, D., Majolino, G., Mariotto, A., Mele, L., Melone, C., Punta, B., Rossi, F., Trotta, V., Venuti, Connection between the vibrational dynamics and the cross-linking properties in cyclodextrinsbased polymers, J. Raman Spectrosc. 44 (2013) 1457.

[52] G., Perale, F., Rossi, M., Santoro, P., Marchetti, A., Mele, F., Castiglione, E., Raffa, and M., Masi, Drug release from hydrogels: A new understanding of transport phenomena, J. Biomed. Nanotechnol. 7 (2011) 1.

[53] F., Rossi, F., Castiglione, M., Ferro, P., Marchini, E., Mauri, M., Moioli, A., Mele, M., Masi, Drug-polymer interactions in hydrogel-based drug-delivery systems: an experimental and theoretical study, Chem. Phys. Chem. 16 (2015) 2818. 
[54] M., Santoro, P., Marchetti, F., Rossi, G., Perale, F., Castiglione, A., Mele, M., Masi, Smart approach to evaluate drug diffusivity in injectable Agar-Carbomer hydrogels for drug delivery, J. Phys. Chem. B 115 (2011) 2503.

[55] F., Rossi, F., Castiglione, M., Ferro, M., Moioli, A., Mele, M., Masi, Drug-Drug interactions in hydrogel systems: an experimental and model study, Chem. Phys. Chem. 17 (2016) 1615.

[56] F., Castiglione, M., Casalegno, M., Ferro, F., Rossi, G. Raos, and A. Mele, Evidence of superdiffusive nanoscale motion in anionic polymeric hydrogels: Analysis of PGSE- NMR data and comparison with drug release properties, J. of Controlled Release, 305 (2019) 110. 


\title{
Chapter 5
}

The role of first principles mathematical modeling in the nanomedicine field

Tommaso Casalini

Institute for Chemical and Bioengineering, Department of Chemistry and Applied Bioscience, ETH Zurich, Vladimir - Prelog - Weg 1 - 5/10, 8093 Zurich, Switzerland;

Biomaterials Laboratory, Institute for Mechanical Engineering and Materials Technology, SUPSI, Via Cantonale 2C, 6928 Manno, Switzerland;

\begin{abstract}
: the advent of nanomedicine brought a new paradigm in the biomedical field, introducing novel health care solutions based on nanosized carriers. In particular, nanoparticles attracted a lot of interest as devices for drug and vaccine delivery, imaging and diagnostic purposes. By virtue of their size (which ranges from 1 to $1000 \mathrm{~nm}$ and thus comparable to molecules of biological interest, like proteins), such nanocarriers exhibit some peculiar features that make nanomedicine a unique discipline. Indeed, nanoparticles can spread all over the body (including cells and organelles) and when injected in body fluids they interact with biological components (such as proteins, carbohydrates, small molecules, et cetera). This aspect cannot be underestimated, since it can lead to unpredictable toxic effects as well as substantial deviations from the expected cellular uptake. In addition, nanoparticles must be designed so that they mainly target the area of interest (e.g., cancer) avoiding accumulation and drug release in healthy organs. This chapter aims at answering the following question: how can first principles mathematical modeling deal with the new challenges and issues introduced by nanomedicine?
\end{abstract}

Keywords: mathematical modeling; molecular dynamics; protein corona; targeting; drug delivery;

\subsection{The new challenges introduced by nanomedicine}

The conception and the synthesis of nanosized devices such as dendrimers, nanoparticles and liposomes has shaped a new discipline usually referred as nanomedicine. Because of their size (between 1 and $1000 \mathrm{~nm}$ ), such nanovectors are able to spread all over the body and to penetrate inside cells and organelles. Effective nanocarriers must be designed so that they remain in the systemic circulation for an adequate time span to perform their task and to target the area of interest, avoiding accumulation in healthy organs. In principle this allows, e.g., releasing an active compound mainly in cancer cells at a desired rate, maintaining a therapeutic concentration for a given period and at the same time minimizing the amount of administered drug, with a positive impact on side effects and costs. Amongst the proposed and discussed nanosized devices, nanoparticles found extensive applications as platforms for the targeted delivery of drugs and vaccines, image contrast agents and for diagnostic purposes ${ }^{1}$. However, the peculiarity of nanomedicine did not bring only new heath care solutions but also novel challenges to face.

When nanoparticles are injected in biological fluids (blood, plasma, interstitial fluids) they interact with the biological components present in the surrounding medium. This aspect has remarkable consequences, since such interactions affect not only in vivo biodistribution and clearance but can provide toxic effects that cannot be easily predicted $a$ priori.

The interactions between nanoparticle surface and biological components lead to the so-called nanobio interface ${ }^{2}$. The fundamental driving forces behind nano-bio interface have been already identified and discussed in literature and are essentially related to Van der Waals and electrostatic interactions as well as hydrophobic and depletion effects. In this regard, the challenge arises from a correct rationalization of the interconnections and the synergistic effects of the involved phenomena, which are strictly dependent on the specific feature of the system. 
One of the most relevant outcomes is the biomolecular corona, that is, the formation of a layer of adsorbed proteins and other biomolecules on the nanoparticle surface ${ }^{3,4}$. The corona is constituted by a heterogeneous mixture of different components with high affinity for the surface of the nanoparticle; therefore, the relative abundances of a given component in the corona and in the surrounding fluid can be very different. The formation of the corona is a very fast process (in the order of milliseconds) and strongly depends on environmental conditions such as ionic strength, $\mathrm{pH}$, biomolecules concentration, et cetera. Consequently, predicting the composition of biomolecular corona is very challenging both in situ and in vitro.

On top of that, the formation of the corona can lead to unpredictable adverse effects. Adsorbed proteins can experience substantial conformational changes because of the interactions with the surface, which can cause protein aggregation and fibrillation, loss of activity and exposure of new antigenic epitopes.

As mentioned, a properly designed nanoparticle must be able to selectively target the tissue of interest (e.g., to penetrate mainly within a tumor), lessening the accumulation in healthy organs. A common strategy to perform this task is the active targeting; briefly, nanoparticle surface is functionalized with suitable ligands that specifically interact with receptors, which are overexpressed by the cells of the target of interest. The ligands must thus be available on nanoparticle surface and, if peptides are employed, their interactions with the surface should not imply conformational changes that can compromise their binding with the receptor.

To perform their task, nanoparticles must also cross some barriers (such as the blood brain barrier (BBB)), diffuse within a tumor (if anticancer drugs are loaded) and release the active compound at a given rate, so that a suitable therapeutic concentration can be maintained for an adequate time span. Mathematical modeling constitutes a valuable support to address these issues, by providing a fundamental understanding of the most important phenomena and optimize nanoparticles design and formulation.

The described challenges exhibit different characteristic time and length scales and this has an obvious impact on the modeling approach and thus on model outcomes. The formation of the biomolecular corona and the targeting (through the ligand/receptor binding) have a characteristic time scale of milliseconds and a characteristic length scale of nanometers, due to the fundamental interactions at atomic level that govern system behavior. Approaches like molecular dynamics simulations, with their resolution at molecular scale, represent an attractive choice.

On the other hand, nanoparticles clearance and diffusion within barriers and tissues, as well as drug release over time, are characterized by higher time (seconds to hours) and length scales (up to centimeters), for which macroscale models, i.e. fundamental conservation equations, are the appropriate approach.

After a brief theoretical background concerning the commonly adopted computational technique, the advantages and the opportunities of mathematical modeling in the nanomedicine field are discussed through selected examples from scientific literature.

For the sake of clarity and completeness, the here presented approaches deal with first principles mathematical modeling and methods that belong to data science (machine learning, artificial intelligence) are not covered. Machine learning is experiencing an increasing use also in nanomedicine and can be coupled to first principle models leading to the so-called hybrid models, which combine the advantages of both approaches. The interested reader is referred to ad hoc reviews ${ }^{5,6}$.

\subsection{Modeling approaches}

\subsubsection{An introduction to molecular modeling}

Broadly speaking, molecular modeling can be rationalized as the combination of two elements: a molecular model and a suitable computational technique to study molecular motion ${ }^{7}$.

The molecular model represents how the system is rationalized, simplified and represented in order to perform meaningful simulations. This is an essential step, due to the limited number of atoms that 
can be included in a simulation (up to $10^{5}-10^{6}$, according to the available computational resources and infrastructures).

In this regard, there are essentially two approaches that can be adopted to represent the system in a molecular simulation. In full atomistic (FA) models, all atoms are explicitly included as the smallest constitutive units of the system. Coarse-grained (CG) models lose the atomic detail by embedding groups of atoms into beads, which are representative of the enclosed atoms in terms of charge, polarity, hydrogen bonding, et cetera. Such simplification is mandatory for those systems whose investigation at atomic scale is not affordable because of an excessive computational effort due to the intrinsic high time and/or length scales of the phenomena of interest. Anyway, if a coarse-grained model is able to keep the main features of the system (charge, balance between hydrophobic/hydrophilic effects, et cetera), it constitutes a powerful tool to perform meaningful simulations with an affordable computational effort. On the other side, also the drawbacks of CG models, due to their intrinsic limits, should be taken into account: indeed, strong electrostatic interactions, anisotropic interactions (like hydrogen bonding) and solvation effects are poorly accounted for ${ }^{8}$. In addition, changes in protein secondary structures are still challenging to describe. For the sake of completeness, it must be mentioned that there are more detailed representations, where the smallest constitutive units are not atoms themselves but electrons; these models are usually treated with quantum chemistry-based methods, which are seldom employed due to the low computational efficiency that strongly limits the maximum number of atoms present in the system (few hundredths). The concept of molecular model also includes those simplifications, which cannot be avoided when complex systems are investigated, either with full atomistic or coarse-grained representations. The adsorption of a protein on a nanoparticle surface is usually unfeasible due to the system size (with the exception of very small particles, whose diameter ranges between 1 and $10 \mathrm{~nm}$ ). A common simplification is approximating the system as a protein that adsorbs on a flat surface with a suitable thickness. On the one side, the phenomena of interest take place at solvent/nanoparticle interface, while the bulk of nanoparticle is not of interest. On the other side, if protein size (hydrodynamic or gyration radius) is much smaller than nanoparticle size, curvature effects are negligible. However, if characteristic sizes are comparable this simplification is no longer acceptable and curvature effects must be accounted for.

As mentioned, the second component of molecular modeling is a suitable computational technique, which allows characterizing the dynamics, the energetics and obtaining a conformational sampling of the system. This topic is covered in the following paragraphs.

\subsubsection{Molecular dynamics}

Molecular dynamics (MD) simulations are the method of choice for FA models. The system is represented as spheres mutually interacting according to a potential energy function called force field

9 . Dynamics are propagated by integrating Newton equation of motion (eq. 1):

$m_{i} \frac{d^{2} r_{i}}{d t^{2}}=F_{i}=-\nabla U(r)$

where $m_{i}$ is the mass of the $\mathrm{i}$-th atom, $r_{i}$ are the spatial coordinates of the $\mathrm{i}$-th atom, $\mathrm{t}$ is time, $F_{i}$ is the force acting on the i-th atom and $U(r)$ is the force field (FF), which is function of the atomic coordinates of all atoms present in the system $r$. The main assumption behind MD is that the use of classical mechanics is a reasonable approximation if quantum effects are not relevant ${ }^{9}$.

Force fields account for long-range interactions (electrostatic, Van der Waals) as well as interactions involving covalent bonds (i.e., bonds, angles, dihedrals). They are parameterized in order to best reproduce minimum energy conformations obtained through quantum mechanics calculations at high level of theory and/or experimental data (hydration enthalpies, structural parameters from nuclear magnetic resonance (NMR), et cetera). There are both "general purposes" force fields, usually chosen to simulate small ligands, as well as FF tailored and parameterized for specific categories of molecules, like proteins, lipids, polymers, carbohydrates, et cetera ${ }^{10}$. The force field must be wisely 
chosen, because the reliability of the results is strongly dependent on the accuracy of the adopted force field.

MD simulations provide a detail at molecular level and can take into account environmental effects by including explicit solvent molecules and ions (or other solute molecules) at a given concentration. The main output of a standard simulation is the conformational sampling of the system contained in the molecular trajectory, whose subsequent post-processing provides insights concerning molecular conformations or interaction energies.

MD simulations do not explicitly consider electrons (charges are accounted for by assigning a partial atomic charge to each atom), therefore phenomena like chemical reactions, excited states and dynamic protonation/deprotonation in solution cannot be simulated with standard protocols. Such investigation would require quantum mechanics/molecular mechanics (QM/MM) methods (for chemical reactions) or Constant $\mathrm{pH}$ simulations (for protonation/deprotonation), whose description is outside the purpose of this chapter.

\subsubsection{Coarse-grained simulations}

As mentioned, when the system of interest is too complex for a simulation at atomic level (because of, e.g., the involved time and length scales), CG models represent a good compromise between a reasonable computational effort and meaningful simulations. It is also worth mentioning that the coarse-graining procedure can be performed at different levels, i.e., a bead can be representative of a group of atoms, a protein or a nanoparticle, according to the phenomenon of interest. The solvent can be taken into account explicitly (by adding beads representative of groups of solvent molecules) or implicitly, by tuning the interactions between beads.

There are different computational techniques to run a simulation a CG scale.

Coarse-grained molecular dynamics simulations are still based on the integration of Newton equation of motion by adopting a suitable force field where the interactions between beads are consistently parameterized. In this regard, MARTINI force field emerged as an interesting choice, due to the straightforward coarse-graining procedure and the validated parameters of the $\mathrm{FF}^{11,12}$. Indeed, MARTINI implements a library of beads, divided in categories and subcategories according to charge, polarity and hydrogen bond capability. Each bead encloses a group of 3 or 4 heavy atoms and are already parameterized in order to best reproduce thermodynamic properties such as free energy of hydration, free energy of vaporization and partitioning between water and other solvents.

Examples of MARTINI coarse-graining are provided in Figure 1.

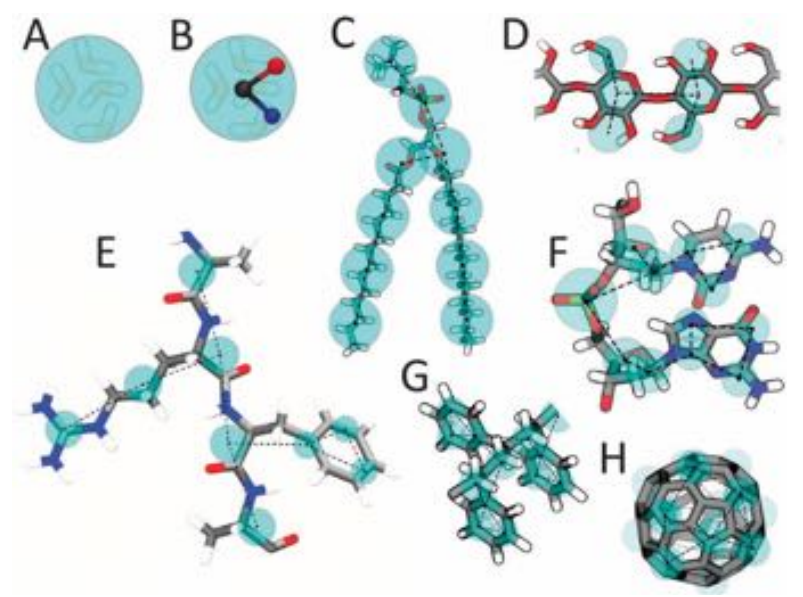

Figure 1. Examples of MARTINI coarse-graining. Water bead containing four water molecules (A). Polarizable water bead with embedded charges (B). DMPC lipid (C). Polysaccharide (D). Peptide (E). DNA fragment (F). Polystyrene fragment (G). Fullerene (H). Reproduced with permission from Marrink and coworkers ${ }^{12}$. Copyright The Royal Society of Chemistry, 2013. 
Brownian Dynamics (BD) simulations are based on Langevin equation; Newton equation of motion is numerically integrated considering three different contributions: a systematic force (due to beads/beads interactions), a frictional force (that depends on velocity and accounts for the friction with solvent) and a random force (which acts as a white noise and determines Brownian motion). In particular, BD simulations constitute the so-called overdamped Langevin dynamics, where the inertial term is neglected and set equal to zero ${ }^{13}$.

Dissipative Particle Dynamics (DPD) constitutes another suitable method for simulations at CG level. The starting point is still the integration of Newton equation of motion, but according to DPD formalism, each bead experiences three different forces: a conservative one due to beads interaction potential, a dissipative one and a random one. DPD represents the minimal model that can account for viscous forces and thermal noise ${ }^{14}$.

In Monte Carlo simulations, beads still interact according to a suitable parameterized potential, but their motion is described through a Metropolis algorithm. Briefly, at a generic simulation step $n$ the value of the potential energy $U_{n}$ is computed. In the subsequent step $n+1$, randomly-chosen particles attempt to perform a displacement $\Delta r$ and a new value of potential energy $U_{n+1}$ is computed. The probability to accept the displacement is computed as follows:

$\operatorname{acc}(n \rightarrow n+1)=\min \left(1, \exp \left[-\frac{1}{k_{B} T}\left(U_{n+1}-U_{n}\right)\right]\right)$

The displacement is accepted or rejected according to a random number $x$, obtained from a uniform distribution in the interval $[0,1]$. The movement is accepted if the probability computed through eq. 2 is higher or equal than $x$ and rejected otherwise ${ }^{9}$.

\subsubsection{Enhanced sampling methods}

Some phenomena occur at molecular scale but with a characteristic time scale that is much higher than the one accessible to an affordable simulation at either FA or CG scale. A typical example is constituted by protein folding, which occurs in a time period that ranges from milliseconds to seconds and therefore could not be investigated with a standard simulation protocol. Another typical case, more related to the content of this chapter, is constituted by the conformational changes of a protein resulting from its adsorption on nanoparticle surface.

Broadly speaking, this has been explained considering the presence of metastable states separated by free energy barriers much higher than the thermal energy $k_{B} T$ (where $k_{B}$ is Boltzmann constant and $T$ is absolute temperature), which would be rarely crossed in a simulation at temperature $T$. This issue led to the development of enhanced sampling methods, which promote the crossing of such barriers and thus the transitions between metastable states while assuring a reasonable computational effort. There are essentially three different approaches: increasing the temperature $T$, changing the potential $U(r)$ and introducing a bias potential $V(r)$. A detailed discussion of the theoretical background and the different approaches is beyond the purpose of this chapter and the reader is referred to ad hoc reviews ${ }^{15,16}$.

Among the different methods, Well-Tempered Metadynamics (WTM) and its variants attracted a lot of interest ${ }^{17}$. Broadly speaking, WTM and WTM-based methods allow recovering the free energy of the system of interest as a function of few relevant degrees of freedom, commonly referred as collective variables $(\mathrm{CV})$; this is carried out by adding a time dependent bias potential (third approach). CV are function of atomic coordinates with different degrees of complexity, since they can vary from a simple atomic distance to more complicate quantities such as the number of hydrogen bonds or hydrophobic contacts, electrostatic interaction energy or the content of alpha helix or beta sheet in a protein. The chosen collective variables must be able to discriminate metastable states and should be representative of the transition mechanism as well.

Phenomena of interest, such as protein conformational changes, may require many $\mathrm{CV}$; although conceptually feasible, this introduces some issues such a drop in computational efficiency, a nontrivial interpretation of the results and a difficult convergence of the free energy profile. 
This led to the development of different WTM-based methods, namely Bias Exchange Metadynamics $(B E M D)^{18}$, Parallel Tempering Metadynamics (PTMD) ${ }^{19}$ and Parallel Tempering Metadynamics in the Well-Tempered Ensemble (PTMD-WTE) ${ }^{20}$, in order to alleviate such issues. The interested readers are referred to the corresponding papers for a detailed discussion of the methods and their theoretical basis.

\subsubsection{Macroscale models}

Macroscale models are based on fundamental mass and momentum conservation equations. Energy conservation equation is seldom employed, since the systems under investigation can be reasonably assumed in isothermal conditions. The characteristic time and length scales are seconds to hours and centimeters to meters, respectively.

A typical application of macroscale models in nanomedicine is the investigation of drug release rate from nanoparticles and their transport and distribution in tumors and/or in the human body.

Focusing on drug release, the starting point is usually is the diffusion equation:

$\frac{\partial C}{\partial t}=\nabla(D \nabla C)$

where $C$ is drug concentration, $t$ is time and $D$ is the diffusion coefficient of the drug in the nanoparticle. Equation 3 is written in a general form, where the diffusion coefficient can vary both in time and space and diffusion can take place along all considered spatial coordinates. Focusing on particles, eq. 3 is usually written is spherical coordinates and only radial coordinate is considered, since radius is the characteristic diffusion length. Diffusion equation can be solved with suitable initial and boundary conditions. The initial concentration of drug is known and it is assumed that the active compound is initially uniformly distributed in the particle. Focusing on boundary conditions, the symmetry of concentration profile at particle center and a fixed concentration value or the presence of mass transfer resistances (through the continuity of mass fluxes) are usually assumed ${ }^{21}$. Eq. 3 is usually solved numerically but it is worth mentioning that, under some simplifying assumptions, analytical solutions are available in literature ${ }^{21}$.

The challenge lies in a reliable estimation of the diffusion coefficient, which, in principle, can depend on several variables such as swelling, polymer molecular weight, polymer and drug concentration, et cetera. Currently, there are many established modeling approaches for the estimation of diffusivities in polymers and gels, whose strong and weak points have been already discussed exhaustively in scientific literature. The interested reader is thus referred to specific reviews ${ }^{22,23}$.

An analogous strategy (i.e., the solution of diffusion equation) is also employed for computing the distribution of nanoparticles in cancer and the impact of the released drug on cancer cells, adopting suitable kinetic laws for drug uptake and its growth inhibiting effect. In this case, nanoparticles modeling is often coupled with the mathematical description of tumor growth; the discussion of cancer modeling would require another book chapter and it is thus beyond the purpose of this work, but the interested reader is referred to ad hoc reviews ${ }^{24-26}$.

Mass balances can be also employed to describe the transport of drugs inside blood vessels, for example inside the new capillaries created inside the tumor because of angiogenesis:

$\frac{\partial C}{\partial t}+u \cdot \nabla C=D \nabla^{2} C$

where $u$ is blood flow velocity and $D$ is drug diffusivity in the blood. The formalism is similar to equation 3, while on the left side there is an addition term that accounts for the convective flow. The migration of the drug inside the tumor is taken into account through suitable boundary conditions at vessel/tumor interface or through additional terms in the mass balance. Assuming that blood can be modeled as a Newtonian fluid with a constant viscosity $\mu$ and density $\rho$, momentum conservation equation (Navier-Stokes equation) can be written as follows: 
$\rho\left(\frac{\partial u}{\partial t}+u \nabla u\right)=\mu \nabla^{2} u-\nabla P$

where $P$ is pressure.

Such macroscale models contain many input parameters, such as diffusion coefficients and kinetic constants related to various processes like clearance, metabolism, binding, et cetera. Such parameters have a defined physical meaning and are usually estimated from experimental data, so that model results are as close as possible to experimental outcomes.

In this regard, it is possible to highlight two fundamental aspects.

On the one side, there is a limited availability of experimental data in vitro environment and even less in in vivo environment. This hinders parameters estimation and model validation; consequently, many works remain purely theoretical and are based on parametric simulations.

On the other side, especially in in vivo environment there are many interconnected phenomena to account for. In principle, this can lead to a high number of system - specific input parameters. Their estimation can be challenging and can imply the risk of overfitting, that is, a good agreement between the model results and experimental data even if the mathematical description is wrong. In other words, the agreement is due to the high number of adaptive parameters and not to the consistency of the theoretical framework. Therefore, a good agreement does not mean that the mathematical model is correct; its validity must be assessed with purely predictive simulations that are compared with independent experimental data, which were not employed for parameters estimation.

A robust mathematical model cannot contain all involved phenomena but must account for only the rate - determining processes. This reduces the complexity of the formulaic description and the number of involved parameters, improving the reliability of the results.

Specific modeling frameworks are discussed in section 5.3.3.

\subsection{Applications of mathematical modeling in the nanomedicine field}

\subsubsection{Biomolecular corona}

Simulations at fundamental molecular scale represent the method of choice for the investigation of the early events leading to biomolecular corona, by virtue of their spatial and temporal resolution.

Molecular modeling allows highlighting both the structural changes resulting from adsorption and the main driving forces behind protein/surface interactions. In particular, the spatial resolution ad atomic scale provides some insights that are challenging or impossible to obtain experimentally. Indeed, while circular dichroism spectra show the changes in secondary structure, the computational microscope offered by molecular dynamics provides a detailed picture of structural modifications (in terms of secondary and tertiary structures). In particular, it can indicate which segments of the protein are subjected to structural changes as well as the most important amino acids that drive the adsorption. MD simulations also account for environmental effects (through the addition of explicit solvent molecules, ions, and other solutes) and also for nanoparticle functionalization, through a suitable molecular model of the surface.

As mentioned, the system is rationalized and simplified as a single protein interacting with a flat surface. This is a reasonable approximation if nanoparticle size is much bigger that protein characteristic size and curvature effects can be neglected. The attainment of structural changes may occur over time scales that can be not accessible to standard simulations and enhanced sampling methods are usually needed to obtain meaningful results.

Such system representation also implies that protein/protein interactions are neglected, i.e., simulations deal with extremely dilute protein solutions. Protein/protein interactions and the resulting conformational changes are challenging to be taken into account also with enhanced sampling methods.

The use of simulations at CG scale can alleviate these issues because of the possibility to explore longer time scales and provide interesting insights like input guess structures of protein/protein 
complexes for more detailed simulations at atomic scale. Given the intrinsic limits connected to coarse-graining, an accurate parameterization of the underlying force field is a mandatory requirement.

Simulations are employed for a wide range of systems, such as nanoparticles, carbon nanotubes, dendrimers, graphene sheets, hydroxyapatite and titanium oxide surfaces.

Obtained results must be validated against comparison with experimental data. Protein affinity with the surface can be compared with the experimental outcomes from isothermal titration calorimetry. While a good quantitative comparison is challenging to achieve, the ranking obtained by molecular simulations is usually in good agreement. In other words, simulations are able to discriminate between strong and weak binders. Conformational changes observed through molecular trajectories can be verified, e.g., with circular dichroism (CD) spectra. In some cases, it is possible to compare directly an experimental outcome with the corresponding simulated one, as happens for CD spectra ${ }^{27,28}$.

Chong et al. ${ }^{29}$ studied from both an experimental and a computational point of view the adsorption of the four most abundant plasma proteins (fibrinogen, immunoglobulin, transferrin and serum albumin) on graphene surface (Figure 2A). MD simulations were employed to compute binding affinity and the attainment of structural changes; results were in good agreement with experimental data. $\mathrm{Gu}$ and coworkers ${ }^{30}$ investigated the binding of $\mathrm{MoS}_{2}$ nanoflakes with potassium channel proteins, in order to highlight possible alteration of biological functions and thus the attainment of toxic effects (Figure 2B). Simulation results were supported by experimental data.

A

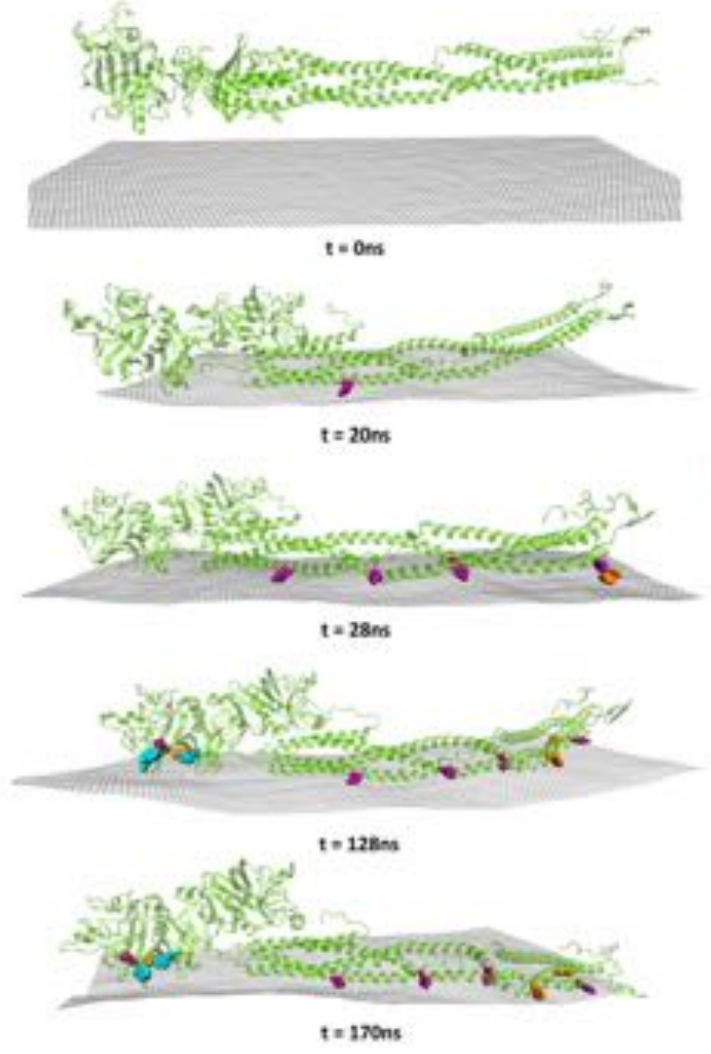

B

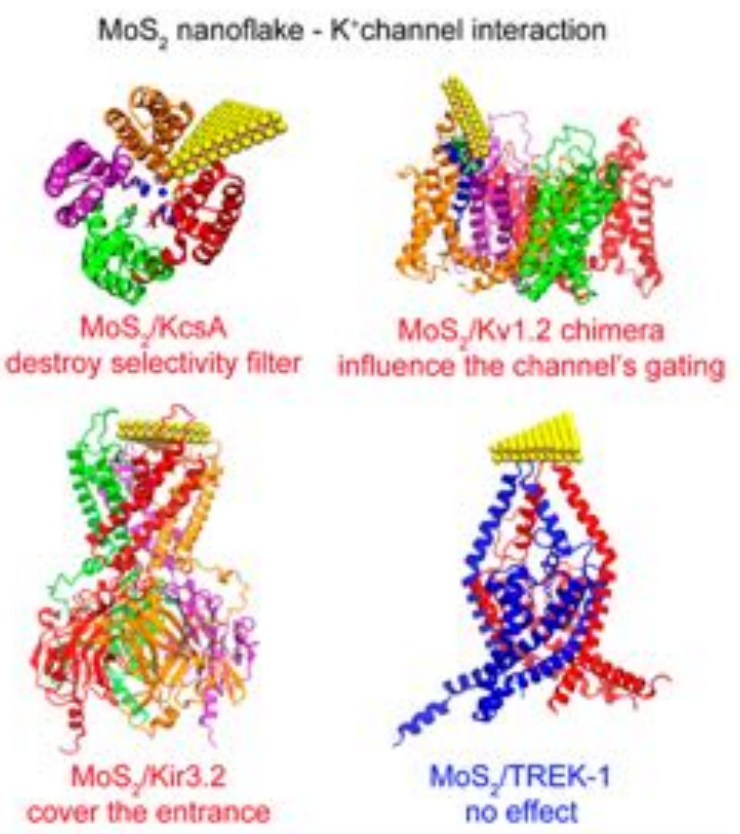

Figure 2. Fibrinogen adsorption on graphene oxide surface at different simulation times. Tyr, Phe and Trp are represented as purple, orange and blue VdW spheres, respectively. Graphene sheet atoms are colored in gray. Reproduced with permission from Chong et al. ${ }^{29}$. Copyright American Chemical Society, 2015 (A). Effect of MoS2 binding to different potassium channel proteins. Reproduced with permission from Gu et al. ${ }^{30}$. Copyright American Chemical Society, 2017 (B). 
Hildebrand and coworkers ${ }^{31}$ investigated the adsorption of the enzyme chymotrypsin on $\mathrm{SiO}_{2} \mathrm{surface}_{\text {, }}$ adopting a Metadynamics-based method. Simulations were in good agreement with CD spectra, which showed a loss in alpha helix content; in particular, calculations highlighted that only one of the two helical segments is affected by loss of secondary structure due to adsorption. In addition, results were employed to compute a theoretical CD spectra, in good agreement with the experimental one. Bellucci et al. ${ }^{32}$ studied the adsorption on a gold surface of the segment $16-22$ of the amyloid $\beta$ peptide, which forms fibrils in water solutions. PTMD simulations allowed identifying the correct conformation of the adsorbed peptide, which was validated by comparing experimental and theoretical sum generation frequency spectra, which were in good agreement each other. On top of that, simulations gave insights concerning the inhibition of fibril formation provided by the addition of gold nanoparticles. Results are summarized in Figure 3.

A
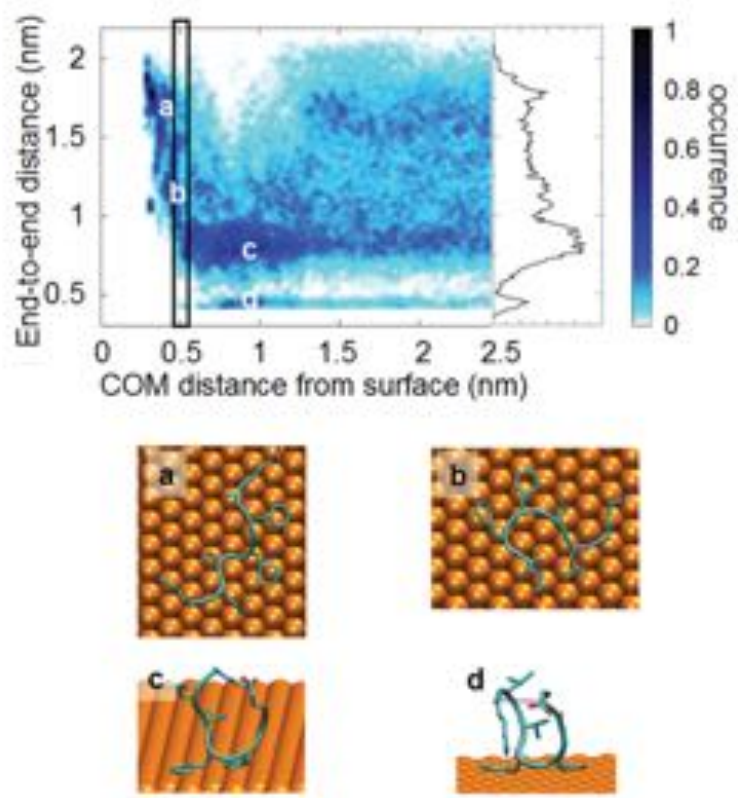
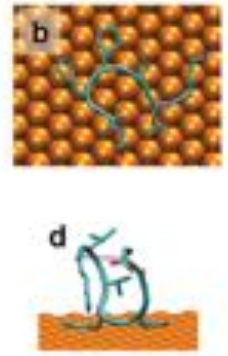

B

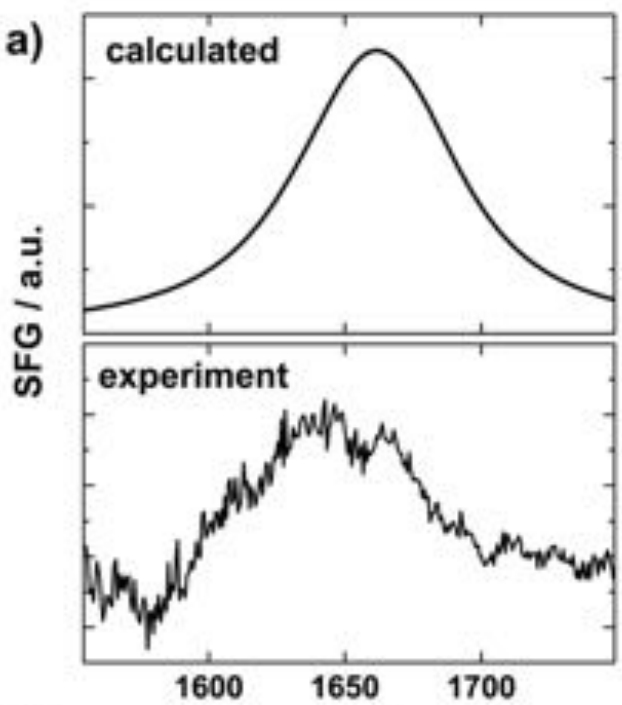

b) IR wavenumber $\mathrm{cm}^{-1}$

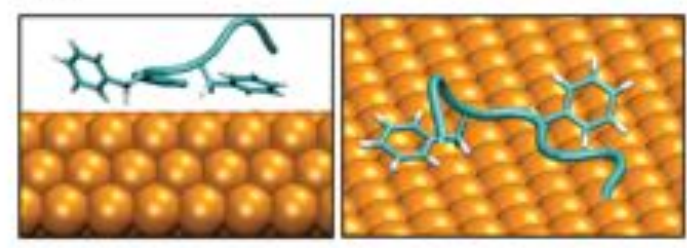

Figure 3. (A) Distribution of peptide end - to - end distance (computed considering terminal C $\alpha$ atoms) as a function of peptide - surface distance. The rectangle identifies the free energy minimum as a function of the peptide - surface distance. The inset represents the distribution of the end-toend distance in the bulk region (COM distance from the surface larger than $1.25 \mathrm{~nm}$ ). Panels a $-\mathrm{d}$ show representative conformation. (B) Comparison between calculated and experimental SFG spectra (a) and simulated structure used for spectra calculation (b). Reproduced with permission from Bellucci and coworkers ${ }^{32}$. Copyright The Royal Society of Chemistry, 2016.

Prakash and coworkers ${ }^{33}$ adopted metadynamics-based methods to investigate the adsorption of GGKGG peptide on silica surface, focusing on the influence of ionic strength and ions charge. The authors systematically analyzed the performances of the computational methods, providing suggestions for the optimal simulation protocol.

$\mathrm{Yu}$ and $\mathrm{Zhou}^{34}$ adopted MARTINI force field for CG simulations in order to highlight the effect of curvature and ionic strength on lysozyme adsorption on silica nanoparticles. The authors found that 
surface curvature has a relevant effect on structural changes, while ionic strength has a moderate influence (Figure 4). The study is purely theoretic and is not supported by experimental data.

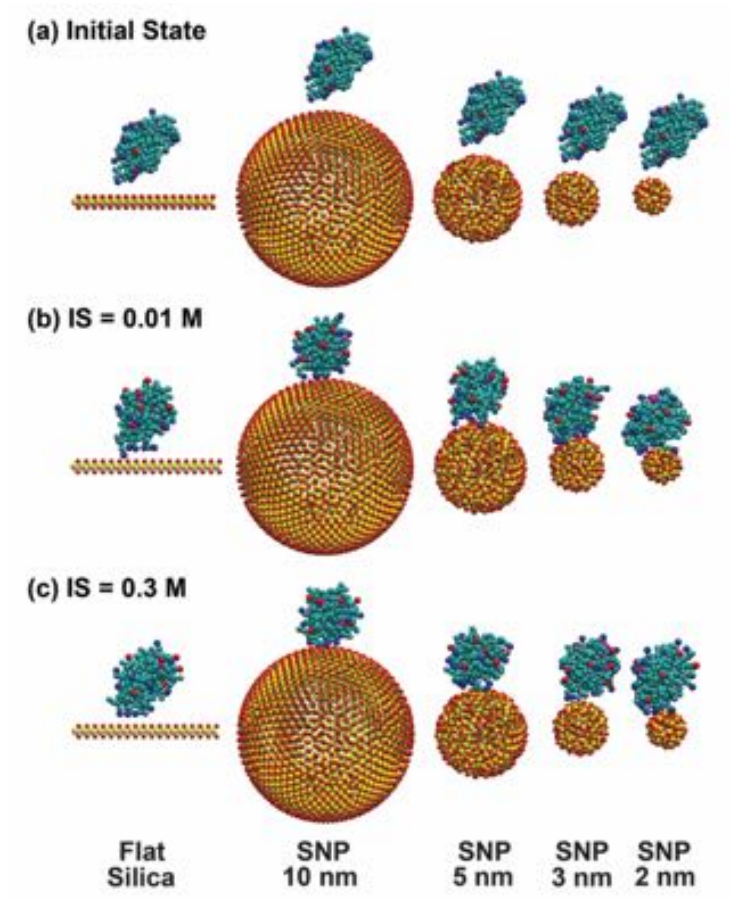

Figure 4. CG model of lysozyme adsorption on silica nanoparticles (SNP) for different values of ionic strength (IS). (a: initial configuration; $b$ and $c$ : representative configurations after CG simulations). Reproduced with permission from $\mathrm{Yu}$ and $\mathrm{Zhou}^{34}$. Copyright The Royal Society of Chemistry, 2016.

Ding and $\mathrm{Ma}^{35}$ employed dissipative particle dynamics to investigate from a theoretical point of view the adsorption of human serum albumin on generic nanoparticles with hydrophobic, hydrophilic and charged surfaces at different $\mathrm{pH}$ and nanoparticle size values. They computed the binding free energy as a function of the centers of mass of the protein and the particle (Figure 5A). Results showed that albumin only binds to hydrophobic and positively charged nanoparticles. The authors also simulated the early events leading to corona formation, computing the number of adsorbed proteins for different value of particle size at physiological $\mathrm{pH}$ (Figure 5C). 
A

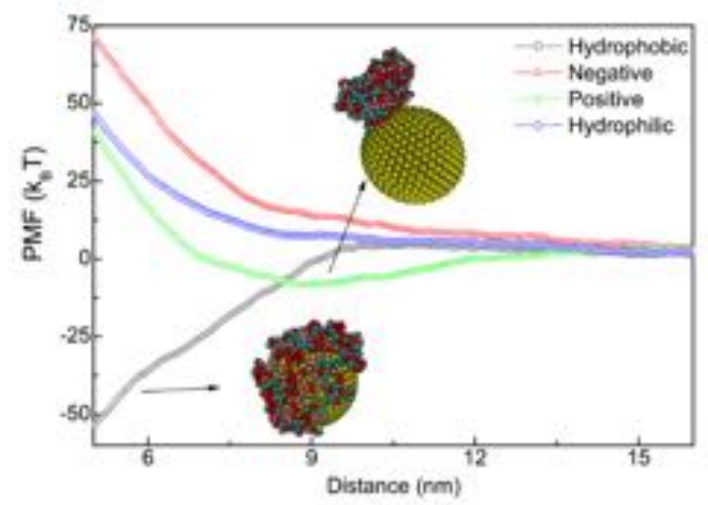

B

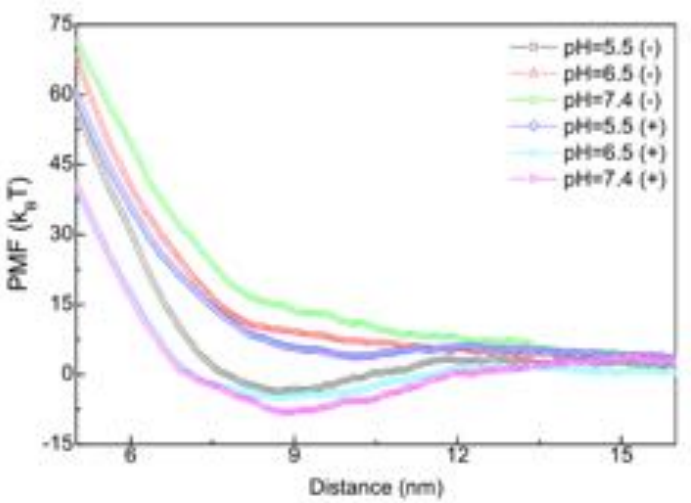

C

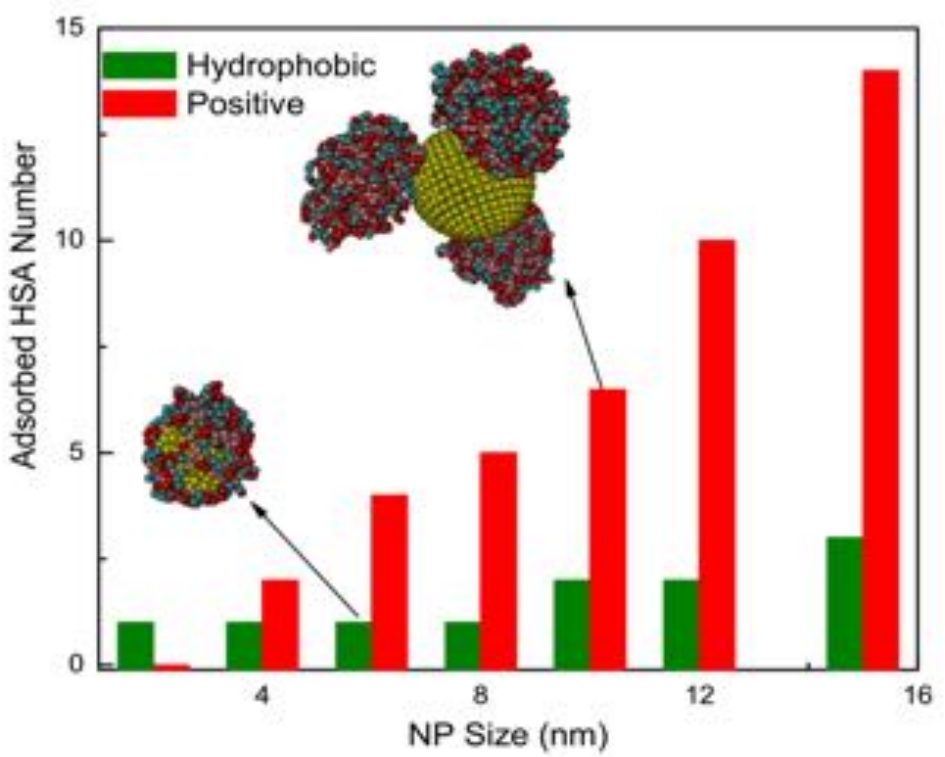

Figure 5. Potential of mean force for HSA binding at $\mathrm{pH} 7.4 \mathrm{on} 10 \mathrm{~nm}$ nanoparticles as a function of protein/nanoparticle COM distance for different surface properties (A). Potential of mean force for HSA binding on $10 \mathrm{~nm}$ charged particles at different $\mathrm{pH}$ values as a function of protein/nanoparticle COM distance (+: positively charged particles; -: negatively charged particles)

(B). Number of adsorbed HSA proteins at $\mathrm{pH} 7.4$ as a function of nanoparticle size and material (C). Reproduced with permission from Ding and $\mathrm{Ma}^{35}$. Copyright Elsevier, 2014.

\subsubsection{Targeting and cellular uptake}

As mentioned, nanoparticles must be able to selectively penetrate and diffuse in the tissue of interest and to minimize their accumulation in healthy organs. A common strategy is the active targeting: nanoparticle surface is decorated with ligands (small molecules, peptides, carbohydrates, et cetera) that specifically interact with receptors that are overexpressed in the diseased area. Simulations at molecular level can highlight the interactions between the ligands and the surface.

Similarly to protein corona simulations, big nanoparticles (100 nm or more) are modeled as flat surfaces while small nanoparticles $(1-5 \mathrm{~nm})$ are entirely included in the simulations. According to the investigated phenomena and system rationalization, a simulation can include a single ligand molecule or randomly distributed ones. If ligand surface density can be estimated experimentally, molecular model can be built accordingly.

In addition, molecular simulations can be employed to investigate the interactions between nanoparticles with cellular membranes and thus the cellular uptake not mediated by specific receptors. 
Cellular membranes constitute a heterogeneous and complex environment due to the presence of transmembrane proteins as well as the different kinds of lipid molecules included in the bilayer and thus simplifications are unavoidable, especially at FA level. Simulations of heterogeneous membranes is hindered by the lack of experimental data needed to validate force field parameters and the long simulation times to reach converged results.

The molecular model usually involves model lipid bilayer made of dioleoylphosphatidylcholine (DOPC) or dipalmitoylphosphatidylcholine (DPPC), usually chosen because of the availability of validated parameters for the force fields. Systems that are more complex involve the presence of cholesterol, but there are also examples of simulations at atomistic level of heterogeneous membranes with many involved compounds.

Model membranes are employed also at CG scale, but there are as well examples of simulations with more complex bilayers aimed at obtaining a better model of a real cellular membrane, thanks to the higher accessible time and length scales. Ingolfsson and coworkers ${ }^{36}$, e.g., adopted MARTINI force field to simulate an idealized mammalian plasma membrane, with 63 different compounds asymmetrically distributed in the two sides of the bilayer.

Anyway, most simulations concerning nanoparticles/membrane interactions are performed at CG level, because of the involved time and length scales ${ }^{7}$.

Capeletti et al. ${ }^{37}$ synthesized silica nanoparticles functionalized with gluconamide moieties, aimed at interacting with the lipopolysaccharide on the surface of the outer membrane of gram-negative bacteria. MD simulations were employed to study the interactions between the targeting moieties (nanoparticles were not included in the molecular model) and the liposaccharide surface.

Biscaglia and coworkers ${ }^{38}$ functionalized PEG polymer of PEGylated gold nanoparticles with GE11 targeting dodecapeptide, which specifically binds to epidermal growth factor receptor. MD simulations showed that a cationic spacer between PEG polymer and the peptide is necessary to assure a good exposure of the targeting moiety, as observed experimentally.

In a subsequent work, Mazzuca et al. ${ }^{39}$ studied the functionalization of gold nanoparticles themselves with GE11 peptide through a suitable cysteine-based linker and investigated the targeting capability both experimentally and theoretically by means of MD simulations.

Liu et al. ${ }^{40}$ designed gold nanoclusters functionalized with three different peptides aimed at targeting Glutathione Peroxidase-1 enzyme. They used MD simulations to study the affinity with the target protein and thus to identify the most promising formulation, which was subsequently experimentally tested in vitro.

$\mathrm{Li}$ and coworkers ${ }^{41}$ performed DPD simulations in order to study from a theoretical point of view the influence of PEG molecular weight $\left(550-5000 \mathrm{~g} \mathrm{~mol}^{-1}\right)$ and grafting density $\left(0.2-1.6\right.$ chains nm$\left.{ }^{-2}\right)$ on $8 \mathrm{~nm}$ nanoparticles, in order to maximize the cellular uptake by identifying the optimal parameters combination. The authors also studied in detail the cellular uptake process and proposed three different phases: membrane bending $(0<\mathrm{t}<122 \mathrm{~ns})$, membrane monolayer protruding $(122<\mathrm{t}<$ $750 \mathrm{~ns}$ ) and equilibrium ( $\mathrm{t}>750 \mathrm{~ns}$ ) (Figure 6). 
A
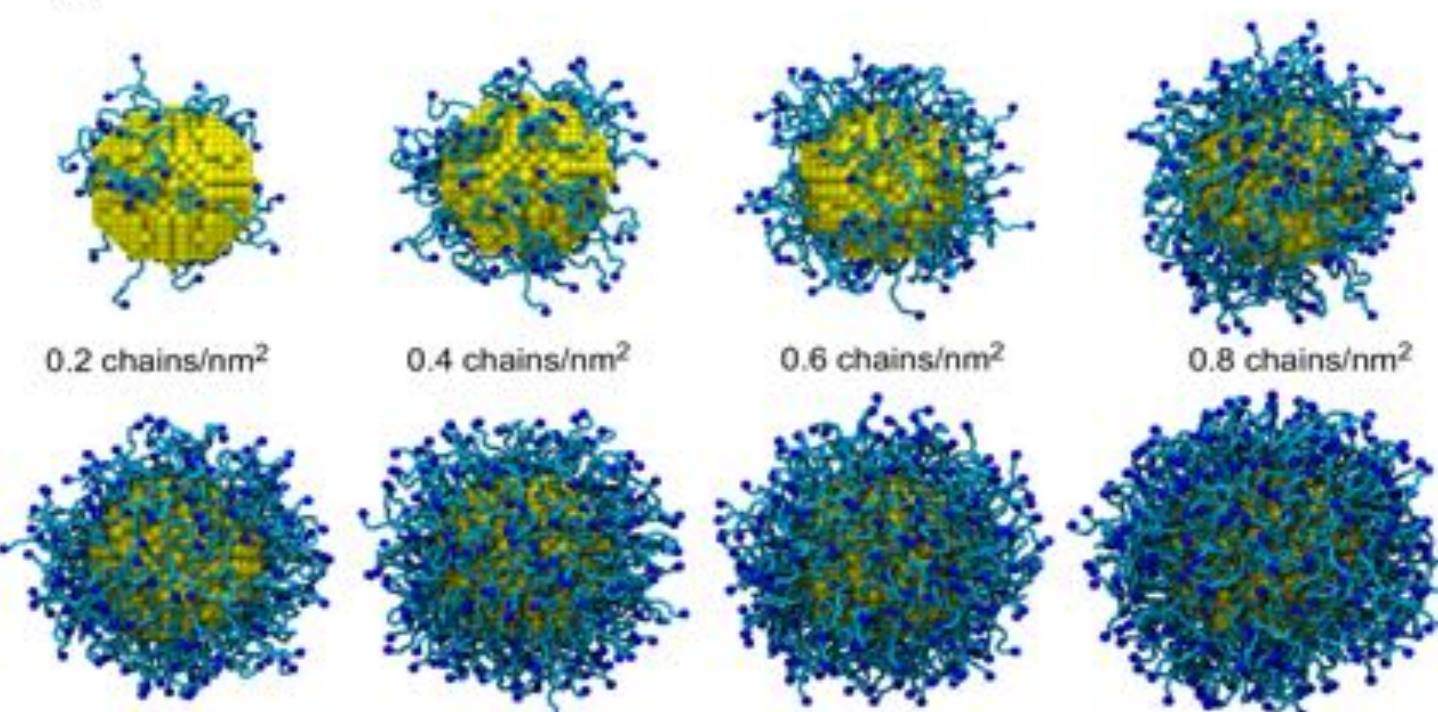

0.4 chains $/ \mathrm{nm}^{2}$

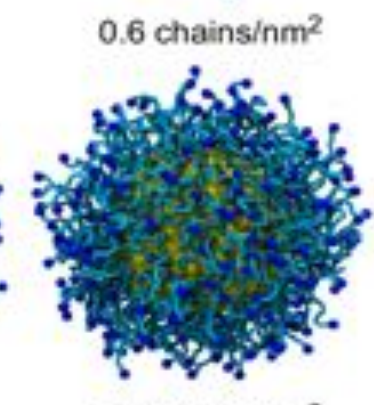

1.0 chainsinm ${ }^{2}$

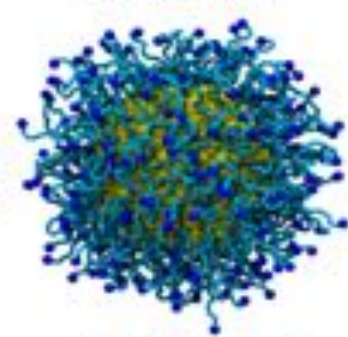

1.2 chains $/ \mathrm{nm}^{2}$

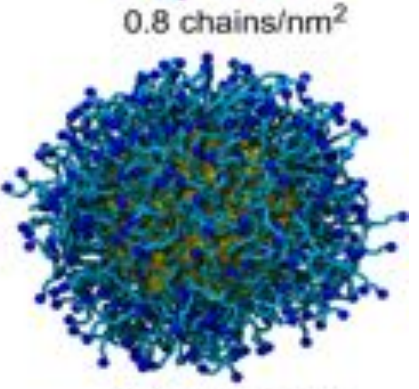

1.6 chains $/ \mathrm{nm}^{2}$

B
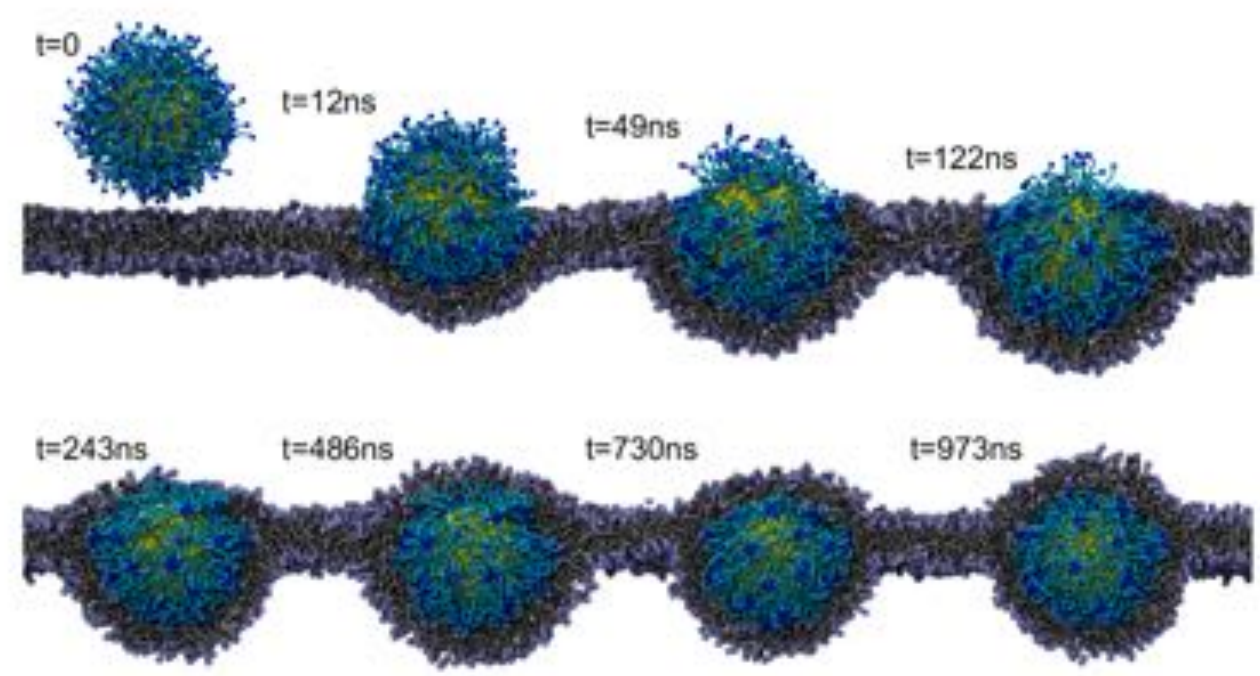

Figure 6. Employed particle models at different grafting density (PEG molecular weight: $838 \mathrm{Da}$ ) (A). Proposed model for particle internalization (grafting density: 1.6 chains $\mathrm{nm}^{-2}$; PEG molecular weight: $838 \mathrm{Da}$ ) (B). Reproduced from Li and coworkers ${ }^{41}$ with permission. Copyright Elsevier, 2014.

Ding and $\mathrm{Ma}^{35}$ adopted DPD to study the influence of a layer of adsorbed human serum albumin on nanoparticle ( $3 \mathrm{~nm}$ in size) permeation in a DPPC model of a cellular membrane. Simulations results showed that the protein layer hinders the cellular uptake due to the interactions with the bilayer, while the naked nanoparticle is able to cross the membrane (Figure 7). 


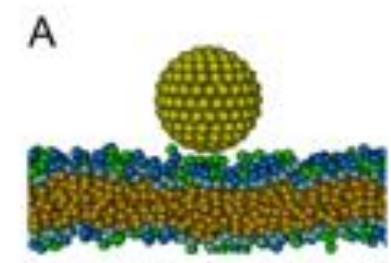

$0.0 \mathrm{Hs}$

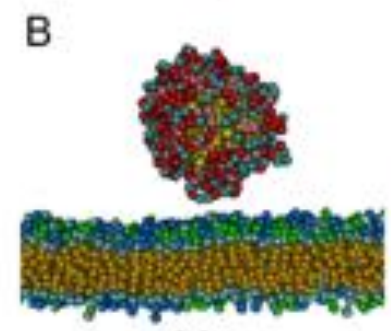

$0.0 \mathrm{ss}$

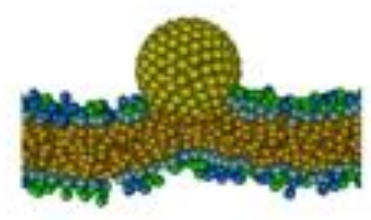

$0.18 \mu \mathrm{s}$

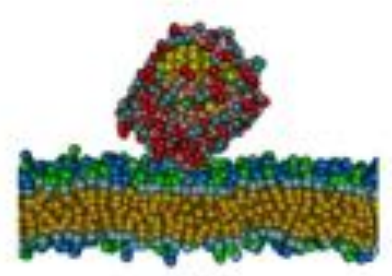

$0.72 \mu \mathrm{s}$

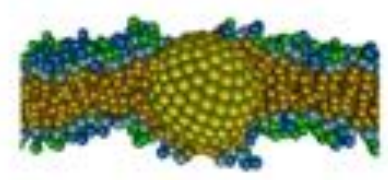

$1,44 \mu \mathrm{s}$

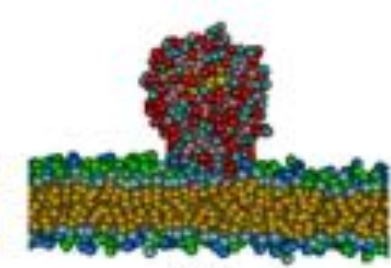

$3.60 \mu \mathrm{s}$
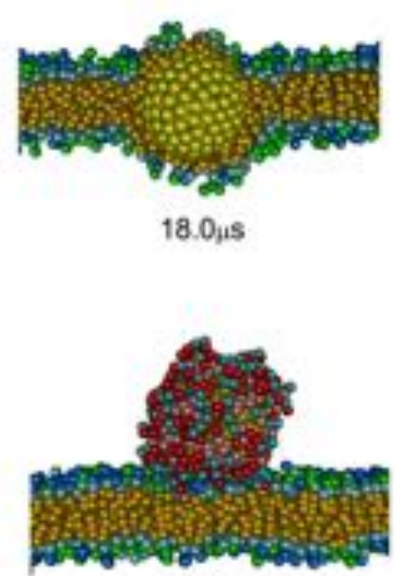

18.0 ps

Figure 7. Hydrophobic particle permeation (A) and HSA - coated particle adhesion (B) on model cellular membrane (pH: 7.4; particle size: $3 \mathrm{~nm}$ ) at different simulation time. Reproduced from Ding and $\mathrm{Ma}^{35}$ with permission. Copyright Elsevier, 2014.

Lunnoo et al..$^{42}$ performed simulations at CG level using MARTINI force field to study the uptake of gold nanodevices. They employed both the more realistic cellular membrane model proposed by Ingolfsson $^{36}$ and a commonly employed simplified model membrane made of DSPC/DSPG. The authors showed that the choice of membrane model is relevant, since the realistic and the simplified models led to different results for what regards the cellular uptake. In more detail, $10 \mathrm{~nm}$ gold nanoparticles experienced an endocytic pathway when the simplified model was chosen (Figure 8A) and a direct translocation across the more realistic membrane (Figure 8B).

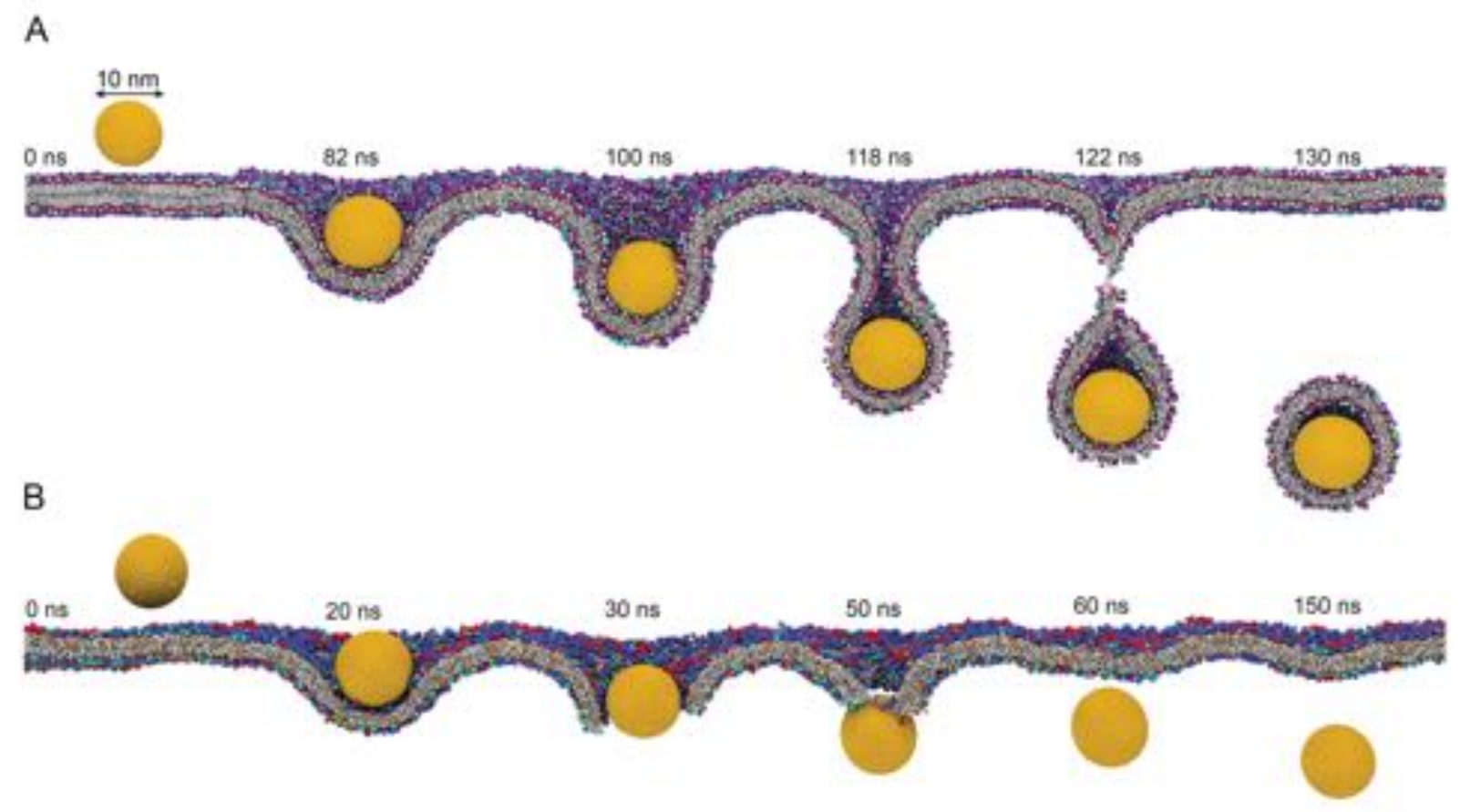

Figure 8. Endocytic pathway for 10nm neutral Au nanoparticle across a model DSPC/DSPG membrane (A). Direct translocation observed a cross a model mammalian cell membrane (B). 
Reproduced with permission from Lunnoo and coworkers ${ }^{42}$. Copyright American Chemical Society, 2019.

\subsubsection{Nanoparticles distribution and drug delivery}

By virtue of their characteristic time and length scales, mass and momentum conservation equations are usually employed to characterize nanoparticles distribution in tissues or cancer environment, drug release from nanoparticles and the impact of the active compound on the disease of interest.

Macroscale models allows understanding the influence of various formulations (in terms of nanoparticle size, surface functionalization, drug release kinetics, et cetera) on the therapeutic effects provided by nanocarriers. A validated model can be employed for predictive simulations, highlighting the most important design parameters and their impact on nanodevices performances, with the consequent optimization of experimental activity and thus saving time and money.

Miller and Frieboes ${ }^{43,44}$ developed a comprehensive model for the study of the release of cisplatin from polylactic-co-glycolic acid and gold nanoparticles, accounting for their distribution in the cancer and the impact of the active compound on tumor growth.

Cancer growth rate $v_{c}$ is expressed as follows:

$v_{c}=-\mu \nabla P+\chi_{E} \nabla E$

where $\mu$ is cell mobility, $P$ is tumor oncotic pressure, $\chi_{E}$ is haptotaxis and $E$ is extracellular matrix (ECM) density. In the modeling framework, time evolution of $E$ is related to ECM production due to angiogenesis, proliferating tumor tissue and degradation through a suitable constitutive law.

Assuming a constant cell density, the overall tumor growth can be related to the rate of volume change:

$\nabla v_{c}=\lambda_{p}$

where $\lambda_{p}$ is net proliferation rate. Diffusion equation at steady state is employed to compute oxygen and nutrient concentration in the tumor.

Focusing on nanoparticle transport, the authors developed a multicompartimental model, identifying an extracellular and a cytosolic compartment. Mass balances can be written as follows:

$\frac{\partial C_{E}}{\partial t} D_{E} \nabla^{2} C_{E}-\frac{k_{E C} C_{E}}{F}+\frac{k_{C E} C_{C}}{F}\left(\frac{10^{6}}{V_{C}}\right)+D(t)$

$\frac{\partial C_{C}}{\partial t}=k_{E C} C_{E}\left(\frac{V_{C}}{10^{6}}\right)-k_{E C} C_{C}-k_{D} C_{C}$

where $C_{E}$ and $C_{C}$ are nanoparticles concentration in extracellular and cytosolic compartments, respectively, $D_{E}$ is nanoparticles diffusion coefficient, $k_{E C}$ is the rate constant related to the transport from extracellular to cytosolic compartment, $F$ is extracellular fraction, $k_{C E}$ is the rate constant related to the transport from cytosolic to extracellular compartment, $k_{D}$ is lysosomal loss, $V_{c}$ is cell volume and $D(t)$ is a forcing function that represents a source of nanoparticles via bolus injection into vasculature. Nanoparticles enter into the extracellular compartment by means of extravasation from the vasculature, which depends on interstitial pressure.

The multicompartmental model was employed also for the released drug; in this case, three compartments were highlighted: cytosolic, extracellular and DNA-bound. Modeling framework also accounts for nanoparticles aggregation.

The model was employed by the authors to investigate the impact of tumor heterogeneity (in terms of viable, necrotic and vessel tissue fraction) on therapeutic efficacy.

Shipley and Chapman ${ }^{45}$ developed a model for fluid and drug transport in vascular tumors. They modeled the interstitium as an isotropic porous medium and adopted Darcy law (eq. 10) and continuity equation (eq. 11) to describe fluid motion therein: 
$u_{t}=-\frac{k}{\mu} \nabla P_{t}$

$\nabla u_{t}=0$

where $k$ is the interstitial permeability, $\mu$ is fluid viscosity and $P_{t}$ is pressure in the interstitium. Fluid flow in capillaries is described by means of Navier-Stokes equation (eq. 12) and continuity equation (eq. 13):

$\rho\left(\frac{\partial u_{c}}{\partial t}+u_{c} \cdot \nabla u_{c}\right)=-\nabla P_{c}+\mu \nabla^{2} u_{c}$

$\nabla u_{c}=0$

where $u_{c}$ is flow velocity and $P_{c}$ is pressure in capillary.

The flux from capillary to interstitium $q_{e}$ is accounted for by means of Starling law:

$q_{e}=L_{p}\left(P_{c}-P_{t}\right) n$

where $L_{p}$ is vascular permeability and $n$ is the unit vector perpendicular to capillary surface.

Drug transport is modeled with convection/diffusion equation, both in the interstitium and in capillary:

$\frac{\partial C}{\partial t}+u \cdot \nabla C=D \nabla^{2} C-\Lambda C$

Where $C$ is drug concentration, $u$ is fluid velocity, $D$ is drug diffusion coefficient and $A$ is drug loss kinetic constant, due to cellular uptake and metabolism. $\Lambda$ is equal to 0 in the capillary and equal to a suitable numerical value in the interstitium.

Sims et al. ${ }^{46}$ developed a mathematical model to characterize the transport of nanoparticles through the female reproductive tract. The authors adopted a compartmental model, identifying three different layers: mucus gel, vaginal epithelium and vaginal stroma and wrote a mass balance for each compartment:

$\frac{\partial C_{M}}{\partial t}=D_{M} \nabla^{2} C_{M}-\left(k_{m}+k_{a}+k_{b d}\right) C_{M}$

$\frac{\partial C_{E}}{\partial t}=D_{E} \nabla^{2} C_{E}-k_{a} C_{E}$

$\frac{\partial C_{S}}{\partial t}=D_{S} \nabla^{2} C_{S}-\left(k_{b}+k_{a}+k_{b d}\right) C_{S}$

where $C_{M}, C_{E}$ and $C_{S}$ are nanoparticles concentration values in mucus, epithelium and stroma, respectively, $D_{M}, D_{E}$ and $D_{S}$ are nanoparticles diffusion coefficient values in mucus, epithelium and stroma, respectively. Focusing on kinetic parameters (first-order rates were assumed), $k_{M}$ is related to the clearance in mucus due to vaginal fluid, $k_{b}$ accounts for the clearance due to vascular and lymphatic system, $k_{b d}$ characterizes the reversible binding between nanoparticles and mucine fiber meshwork, and $k_{a}$ is linked to the probability of self-aggregation, which hinders the transport of nanoparticles. In particular, nanoparticles are assumed to be released from a gel into mucus, according to a first order kinetics:

$C(t)=C_{0} e^{-B t}$

where $C(t)$ is nanoparticle concentration at gel/mucus interface at time $t, C_{0}$ is nanoparticle concentration in the gel before release onset and $B$ is the decay rate constant. 
The authors estimated the parameters from literature and computed nanoparticles concentration as a function of time in each compartment for different formulations, in terms of amount of PEG employed for surface functionalization and different release kinetics from the gel (i.e., for different values of $B$ parameter).

$\mathrm{Xi}$ et al. ${ }^{47}$ employed computational fluid dynamics (CFD) simulations to investigate respiratory airflow and the motion of inhaled and exhaled aerosol tracing particles. The starting point of the study is the hypothesis that the presence of lung cancer can substantially modify the distribution of exhaled particles; in principle, this phenomenon could be exploited as a non-invasive diagnostic tool. Simulations showed that growing bronchial tumors have a remarkable influence on both air velocity field and exhaled particles distribution.

\subsection{Conclusions}

Nanomedicine is experiencing a continuous development and mathematical modeling can constitute a powerful support to improve the understanding and speed up the assessment of new, safer and more effective formulations. Modeling offers a wide range of techniques that allow investigating different shades of the problem of interest, thanks to the different accessible time and length scales. On the one side, methods at molecular scale act as a computational microscope, which allows investigating the phenomena at nano/bio interface at atomic resolution, achieving insights that are challenging to obtain experimentally. A typical application is the study of biomolecular corona, where the main phenomena are known but the rationalization of their synergistic effects is difficult and strictly related to the specific system.

On the other side, mass and momentum conservation equations, fundamental pillars in chemical engineering, are employed to study the distribution of nanoparticles in cancer environment, drug release rate and the impact of active compound of tumor growth, thus assessing the efficacy of the proposed formulation.

Thanks to the advent of new advanced data science techniques, such as machine learning and artificial intelligence, which can support first principles approaches, mathematical modeling can act as a key player in the development of nanomedicine.

\subsection{References}

1. Irvine, D.J. \& Dane, E.L. Enhancing cancer immunotherapy with nanomedicine. Nat Rev Immunol (2020).

2. Nel, A.E., et al. Understanding biophysicochemical interactions at the nano-bio interface. Nat Mater 8, 543-557 (2009).

3. Cedervall, T., et al. Detailed identification of plasma proteins adsorbed on copolymer nanoparticles. Angew Chem Int Edit 46, 5754-5756 (2007).

4. Cedervall, T., et al. Understanding the nanoparticle-protein corona using methods to quantify exchange rates and affinities of proteins for nanoparticles. P Natl Acad Sci USA 104, 20502055 (2007).

5. Yamankurt, G., et al. Exploration of the nanomedicine-design space with high-throughput screening and machine learning. Nat Biomed Eng 3, 318-327 (2019).

6. Jones, D.E., Ghandehari, H. \& Facelli, J.C. A review of the applications of data mining and machine learning for the prediction of biomedical properties of nanoparticles. Comput Meth Prog Bio 132, 93-103 (2016). 
7. Casalini, T., et al. Molecular Modeling for Nanomaterial-Biology Interactions: Opportunities, Challenges, and Perspectives. Front Bioeng Biotechnol 7, 268 (2019).

8. Alessandri, R., et al. Pitfalls of the Martini Model. J Chem Theory Comput 15, 5448-5460 (2019).

9. Frenkel, D. \& Smit, B. Understanding molecular simulation : from algorithms to applications, (Academic Press, San Diego, 2002).

10. Riniker, S. Fixed-Charge Atomistic Force Fields for Molecular Dynamics Simulations in the Condensed Phase: An Overview. J Chem Inf Model 58, 565-578 (2018).

11. Marrink, S.J., Risselada, H.J., Yefimov, S., Tieleman, D.P. \& de Vries, A.H. The MARTINI force field: Coarse grained model for biomolecular simulations. J Phys Chem B 111, 78127824 (2007).

12. Marrink, S.J. \& Tieleman, D.P. Perspective on the Martini model. Chem Soc Rev 42, 68016822 (2013).

13. Chen, J.C. \& Kim, A.S. Brownian Dynamics, Molecular Dynamics, and Monte Carlo modeling of colloidal systems. Adv Colloid Interfac 112, 159-173 (2004).

14. Liu, M.B., Liu, G.R., Zhou, L.W. \& Chang, J.Z. Dissipative Particle Dynamics (DPD): An Overview and Recent Developments. Arch Comput Method E 22, 529-556 (2015).

15. Camilloni, C. \& Pietrucci, F. Advanced simulation techniques for the thermodynamic and kinetic characterization of biological systems. Adv Phys-X 3(2018).

16. Miao, Y.L. \& McCammon, J.A. Unconstrained enhanced sampling for free energy calculations of biomolecules: a review. Mol Simulat 42, 1046-1055 (2016).

17. Valsson, O., Tiwary, P. \& Parrinello, M. Enhancing Important Fluctuations: Rare Events and Metadynamics from a Conceptual Viewpoint. Annu Rev Phys Chem 67, 159-184 (2016).

18. Piana, S. \& Laio, A. A bias-exchange approach to protein folding. J Phys Chem B 111, 45534559 (2007).

19. Bussi, G., Gervasio, F.L., Laio, A. \& Parrinello, M. Free-energy landscape for beta hairpin folding from combined parallel tempering and metadynamics. J Am Chem Soc 128, 1343513441 (2006).

20. Deighan, M., Bonomi, M. \& Pfaendtner, J. Efficient Simulation of Explicitly Solvated Proteins in the Well-Tempered Ensemble. J Chem Theory Comput 8, 2189-2192 (2012).

21. Siepmann, J. \& Siepmann, F. Mathematical modeling of drug delivery. Int J Pharmaceut 364, 328-343 (2008).

22. Casalini, T. \& Perale, G. From Microscale to Macroscale: Nine Orders of Magnitude for a Comprehensive Modeling of Hydrogels for Controlled Drug Delivery. Gels-Basel 5(2019). 
23. Masaro, L. \& Zhu, X.X. Physical models of diffusion for polymer solutions, gels and solids. Prog Polym Sci 24, 731-775 (1999).

24. Deisboeck, T.S., Wang, Z.H., Macklin, P. \& Cristini, V. Multiscale Cancer Modeling. Annu Rev Biomed Eng 13, 127-155 (2011).

25. Anderson, A.R.A. \& Quaranta, V. Integrative mathematical oncology. Nat Rev Cancer 8, 227234 (2008).

26. Chauviere, A.H., et al. Mathematical Oncology: How Are the Mathematical and Physical Sciences Contributing to the War on Breast Cancer? Curr Breast Cancer R 2, 121-129 (2010).

27. Nagy, G., Igaev, M., Jones, N.C., Hoffmann, S.V. \& Grubmuller, H. SESCA: Predicting Circular Dichroism Spectra from Protein Molecular Structures. J Chem Theory Comput 15, 5087-5102 (2019).

28. Michaelis, M., et al. Impact of the Conformational Variability of Oligopeptides on the Computational Prediction of Their CD Spectra. J Phys Chem B 123, 6694-6704 (2019).

29. Chong, Y., et al. Reduced Cytotoxicity of Graphene Nanosheets Mediated by Blood-Protein Coating. Acs Nano 9, 5713-5724 (2015).

30. Gu, Z.L., et al. Exploring the Nanotoxicology of MoS2: A Study on the Interaction of MoS2 Nanoflakes and K+ Channels. Acs Nano 12, 705-717 (2018).

31. Hildebrand, N., et al. Atomistic Details of Chymotrypsin Conformational Changes upon Adsorption on Silica. Acs Biomater Sci Eng 4, 4036-4050 (2018).

32. Bellucci, L., et al. The interaction with gold suppresses fiber-like conformations of the amyloid beta (16-22) peptide. Nanoscale 8, 8737-8748 (2016).

33. Prakash, A., Sprenger, K.G. \& Pfaendtner, J. Essential slow degrees of freedom in proteinsurface simulations: A metadynamics investigation. Biochem Bioph Res Co 498, 274-281 (2018).

34. Yu, G.B. \& Zhou, J. Understanding the curvature effect of silica nanoparticles on lysozyme adsorption orientation and conformation: a mesoscopic coarse-grained simulation study. Phys Chem Chem Phys 18, 23500-23507 (2016).

35. Ding, H.M. \& Ma, Y.Q. Computer simulation of the role of protein corona in cellular delivery of nanoparticles. Biomaterials 35, 8703-8710 (2014).

36. Ingolfsson, H.I., et al. Lipid Organization of the Plasma Membrane. J Am Chem Soc 136, 14554-14559 (2014).

37. Capeletti, L.B., et al. Gram-Negative Bacteria Targeting Mediated by CarbohydrateCarbohydrate Interactions Induced by Surface-Modified Nanoparticles. Adv Funct Mater 29(2019). 
38. Biscaglia, F., et al. Enhanced EGFR Targeting Activity of Plasmonic Nanostructures with Engineered GE11 Peptide. Adv Healthc Mater 6(2017).

39. Mazzuca, C., et al. Understanding the good and poor cell targeting activity of gold nanostructures functionalized with molecular units for the epidermal growth factor receptor. Nanoscale Adv 1, 1970-1979 (2019).

40. Liu, M.Q., et al. Peptide-Au Clusters Induced Tumor Cells Apoptosis via Targeting Glutathione Peroxidase-1: The Molecular Dynamics Assisted Experimental Studies. Sci Rep$U k$ 7(2017).

41. Li, Y., Kroger, M. \& Liu, W.K. Endocytosis of PEGylated nanoparticles accompanied by structural and free energy changes of the grafted polyethylene glycol. Biomaterials 35, 84678478 (2014).

42. Lunnoo, T., Assaeakhajornsak, J. \& Puangmali, T. In Silico Study of Gold Nanoparticle Uptake into a Mammalian Cell: Interplay of Size, Shape, Surface Charge and Aggregation. $J$ Phys Chem C (2019).

43. Miller, H.A. \& Frieboes, H.B. Evaluation of Drug-Loaded Gold Nanoparticle Cytotoxicity as a Function of Tumor Vasculature-Induced Tissue Heterogeneity. Ann Biomed Eng 47, 257271 (2019).

44. Miller, H.A. \& Frieboes, H.B. Pharmacokinetic/Pharmacodynamics Modeling of DrugLoaded PLGA Nanoparticles Targeting Heterogeneously Vascularized Tumor Tissue. Pharm Res-Dordr 36(2019).

45. Shipley, R.J. \& Chapman, S.J. Multiscale Modelling of Fluid and Drug Transport in Vascular Tumours. B Math Biol 72, 1464-1491 (2010).

46. Sims, L.B., Miller, H.A., Halwes, M.E., Steinbach-Rankins, J.M. \& Frieboes, H.B. Modeling of nanoparticle transport through the female reproductive tract for the treatment of infectious diseases. Eur J Pharm Biopharm 138, 37-47 (2019).

47. Xi, J.X., et al. CFD Modeling and Image Analysis of Exhaled Aerosols due to a Growing Bronchial Tumor: towards Non-Invasive Diagnosis and Treatment of Respiratory Obstructive Diseases. Theranostics 5, 443-455 (2015). 
NANOSTRUCURED

SUBSTRATES
FOR

SPECTROSCOPY

Alois Bonifacio

Department of Engineering and Architecture, University of Trieste, Via Valerio 6a, 34127 - Trieste (TS) Italy

\begin{abstract}
After shortly introducing the basics and the most relevant experimental aspects of SERS in the first part, nanostructured metal substrates used as substrates in this technique are discussed in a second part of the chapter. First, general characteristics are considered, and then colloidal and non-colloidal substrates are specifically addressed. Both bottom-up and top-down strategies for substrates preparation are discussed, along with commonly used characterization techniques. A third part is specifically dedicated to how SERS substrates can be used for in-vitro and in-vivo diagnostics and theranostics, stressing the two different "direct" and "indirect" detection strategies, and the role of the nano-bio interface.
\end{abstract}

Key Words: SERS, Raman spectroscopy, nanoparticles, nanostructures, nanomaterials

This chapter is not meant to be an exhaustive, thorough review of the rich variety of metal nanostructures, as available from literature, to be used as Surface-Enhanced Raman Scattering (SERS) substrates. The number and diversity of such substrates are so great that such a task would be a formidable one. For broader and more comprehensive overviews on SERS substrates, the reader is referred to recent reviews (W. Li et al., 2017; Mosier-Boss, 2017) or books on SERS (Prochazka, 2015; Schlücker, 2011).

The purpose of this chapter is rather to introduce the non-specialist reader to most important aspects of metal nanostructures when used as SERS substrates to investigate in-vitro and in-vivo biological samples (e.g. biofluids, cells or tissues) for diagnostic or theranostic purposes. This chapter purposely targets researchers without a direct SERS expertise, addressing a broad audience with a variety of different backgrounds. Thus, theoretical aspects, equations and in-depth technical details will be avoided (the interested reader will be redirected to proper sources), in favor of qualitative explanations.

The chapter consists of three parts: in the first part, the reader is given a very short introduction to basic aspects of SERS, whereas in the second part general aspects of SERS substrates are discussed. The third part specifically addresses aspects relevant to biomedical and diagnostic applications.

\title{
1. AN INTRODUCTION TO SERS
}


Raman spectroscopy is an optical spectroscopic technique based on the homonymous Raman effect (Larkin, 2011; Smith and Dent, 2013; Vandenabeele, 2013): the inelastic scattering of monochromatic light due to molecular vibrations. The effect was theoretically predicted before his experimental discovery (Smekal, 1923), which has been achieved by C.V. Raman in 1928 (Raman and Krishnan, 1928). In the decades after his discovery, all the different theoretical aspects of the effect have been thoroughly described (Long, 2002). Leaving aside the rigorous but complex theoretical treatment, the Raman effect can be qualitatively described in very simple terms. When matter is illuminated with a monochromatic light such as that of a laser beam, most of the scattered light retain the same wavelength as the incident light (i.e. elastic or Rayleigh scattering). However, a very tiny fraction of the scattered light coming out of the sample has different wavelengths because of its interaction with the matter (i.e. Raman scattering). Put differently, the light-matter interaction "shifts" the wavelength of the incoming light to different extents (the units of the "x axis" of Raman spectra are called "Raman shifts"), so that a polychromatic light is generated as a consequence of the energy exchanged between light and matter during the scattering process. These wavelength shifts are modulated by molecular vibrations, so that a Raman spectrum is, like an Infrared (IR) spectrum, a vibrational spectrum, where each band corresponds to a specific type (a "normal mode") of vibration. Inelastic scattering, the physical process behind the Raman effect, however, is very different from the absorption process occurring in IR, and despite both spectroscopies originate from molecular vibrations, IR and Raman spectra are different.

What matters most is that different molecules will vibrate differently: each molecular structure will have its set of "normal modes" of vibrations. A direct consequence of this specificity will be that a vibrational spectrum will be unique for a specific structure, which is the reason why vibrational spectra are sometimes referred to as "molecular fingerprints". The specificity of Raman spectroscopy is of course a distinct advantage in chemical analysis, and this technique, especially with the coming of more compact, performing and accessible instrumentation has been proposed as a solution for many analytical applications. In spite of its many advantages, however, Raman spectroscopy has an intrinsic disadvantage: as the Raman effect is a weak effect, and thus the technique is not very sensitive.

SERS offers a solution to overcome this drawback of the Raman effect by enhancing the intensity of the Raman effect in presence of metal nanostructures (Figure 1). SERS has been serendipitously discovered in the 1970s while studying the behavior of pyridine adsorbed on Ag electrodes (Fleischmann et al., 1974; Jeanmaire and Van Duyne, 1977). After a careful consideration of all the experimental aspects, researchers concluded that the only possible explanation for the spectra observed was that that the intensity of the Raman bands due to the pyridine adsorbed on the roughened Ag electrodes was enhanced by a factor of $10^{6}$ with respect to the normal Raman spectrum observed from pyridine in a bulk solution. Two very important aspects of SERS have been evident since, which define this technique and should be stressed right from the start: i) SERS can boost Raman sensitivity: Raman spectra from very low amounts of substance can in fact 
be observed exploiting SERS, overcoming the intrinsic problem of the poor sensitivity of Raman spectroscopy; ii) SERS is a surface technique: enhanced Raman spectra can be observed only from species adsorbed on (or very close to) a metal surface, so that phenomena occurring at the metal-solution interface (e.g. catalysis) can be studied.

*** Insert Figure $1 * * *$

Caption: Schematic illustration of a SERS experiment

Credit: none (original unpublished figure)

For a long time, the origin and the explanation of the SERS effect has been debated and, in part, it still is (Graham et al., 2017). Experimental findings showed that both the morphology and the chemical nature of the metal surface played a central role, since flat surfaces did not show a significant enhancement and different metals lead to various results (with $\mathrm{Ag}, \mathrm{Au}$ and $\mathrm{Cu}$ being the most effective), but how and why remained a matter of debate. In time, the SERS community slowly built a consensus toward an explanation involving two main mechanisms of enhancement: the so called electromagnetic mechanism (EM) and the chemical mechanism (CM), which could explain the experimental results observed. Details about these mechanisms are not reported here: excellent books address this aspect in detail (Aroca, 2006; Ru and Etchegoin, 2008). It is now generally accepted that the EM is accountable for most of the SERS effect, and that this effect has to do with the presence of localized surface plasmons (i.e. collective oscillation of surface electrons) in metal nanostructures. This description well accommodates the fact that coinage metals such as $\mathrm{Ag}, \mathrm{Au}$ and $\mathrm{Cu}$ display a SERS effect with a laser excitation in the visible or near-infrared range and that the enhancement effect rapidly decays with the distance from the metal surface. This theory also explains why specific surface morphologies involving nanostructures are needed, since plasmonic properties adequate for SERS effect when using visible or near-infrared excitation only arise from metal nanostructures. Nowadays, our grasp of plasmonics allows us to design metal nanostructures tailored to have localized surface plasmons at specific wavelengths, which can be then realized via nanofabrication techniques. Experimental results confirmed theoretical predictions, so that EM is a well-understood and consolidated SERS mechanism.

\subsection{EXPERIMENTAL ASPECTS}

SERS is not an easy, straightforward technique to use. There are many experimental aspects to take into account when planning a SERS experiment, and an experimenter with some experience in SERS will be able to judge what are the best conditions to maximize the chances of success, that is to observe an intense SERS spectrum from a specific analyte. 


\section{Matching substrates and laser wavelengths.}

The first and perhaps most important aspect to take into account is the fact that not all substrates will work with all analytes and with all lasers (Álvarez-Puebla, 2012). The EM theory tells us that, for instance, given a metal surface, the choice of the exciting laser is limited. In the best case, one can design and realize a substrate having exactly the desired characteristics to maximize the match between the surface plasmons frequency and that of the exciting laser, but most often one has to work with a given substrate, or has a limited choice of excitation lasers, and get the most out of it. As a good rule of thumb, Ag substrates work well with a broad range of excitation sources: Ag nanoparticles, for instance, display a SERS effect when excited with lasers having wavelengths in the blue/green region (e.g. using 514/532 nm lasers, but also with $405 / 413 \mathrm{~nm}$ ), but yield intense SERS spectra also upon near infrared excitation (e.g. $785 \mathrm{~nm}$ ) (Álvarez-Puebla, 2012). Au substrates, on the other hand, depending on their characteristics, may work well upon red (e.g. $633 \mathrm{~nm}$ ) or near-infrared (e.g. 785/830 nm) excitation.

\section{Chemical nature of the analyte}

Of utmost importance is also that the analyte should be close to the nanostructured metal surface. Ideally, it should be in direct contact with the metal surface (i.e. physisorbed or chemisorbed). An experienced SERS spectroscopist will predict if such interaction has good chances to occur just by looking at the molecular structure of the analyte. Thiols, amines, $\mathrm{N}$ containing heterocyclic compounds and carboxylic acids do have a strong interaction with $\mathrm{Ag}$ and $\mathrm{Au}$ substrates, and thus they are expected to yield intense SERS spectra.

Sometimes a direct interaction is difficult or impossible. Non-polar molecules lacking the functional groups listed above or carbohydrates, for instance, will not have a strong interaction with Ag or Au surfaces, and SERS spectra of those kind of molecules are notoriously difficult to get, unless chemical (e.g. chemical bonding to an already adsorbed species) or physical methods (e.g. electrostatic interaction) are used to "attract" these analytes close to the surface.

A special attention should be paid to electrostatic charges. Depending on the conditions (e.g. pH of the environment, adsorbates already present on the substrate) the metal surface and the analyte might have definite electrostatic charges. For instance, citrate-reduced Au and Ag nanoparticles, a kind of very common and widely used SERS substrates, have citrate molecules with negatively-charged carboxylate groups adsorbed on the metal surface, conferring an overall negative charge to the surface. With such substrates it will be very difficult to get a SERS signal out of analytes having a definite negative charge. This is one of the reasons why in SERS experiments $\mathrm{pH}$ should be carefully controlled and checked, since a slight change in the $\mathrm{pH}$ might dramatically influence the analyte-substrate interaction, and thus the SERS spectrum generated. 
In general, in view of the subtle dependence on the specific analyte-metal interaction, of the influence of the environmental conditions and on the wavelength-metal matching, it can be stated that no such thing as a "universal" SERS substrate exists, which can be generally used with all possible analytes. Each analyte, or even better, each analytical problem requires a specific SERS solution, which is one of the reasons making SERS a tricky technique to be used by non-experts.

\section{Environmental conditions}

In general, environmental conditions such as $\mathrm{pH}$ and ionic strength (i.e. concentration of charged species in solution) can have a dramatic impact on a SERS experiment, not only for the analyte-substrate interaction but also for the substrate itself. In the case of $\mathrm{Au}$ and $\mathrm{Ag}$ colloids, for instance, the formation of nanoparticles aggregates is functional to the generation of adequate plasmonic nanostructures (Fraire et al., 2013; Zhang et al., 2015). Such aggregates can be formed by increasing the ionic strength of the environment, which will shield the surface charges responsible of the colloid stability, leading to nanoparticles aggregation. Sometimes the ionic strength is increased by the addition of salts to the colloid, but sometimes the analyte solution has already the necessary ionic strength to lead to nanoparticle aggregation. Working with analytes in buffered saline solutions rather than in water is often desirable in SERS experiments using colloidal $\mathrm{Ag}$ or $\mathrm{Au}$ as substrates, since $\mathrm{pH}$ is well defined and the ionic strength is often already high enough to induce nanoparticle aggregation.

\section{Interference from other species}

Often, more than one species is present together with the analyte in the solution: these can be buffering species, salts or other molecules, as in the case of "real", chemical complex samples such as biofluids. In these cases, the problem of competing species might arise. Species other than the analyte might compete with the analyte itself for the metal surface, so that the SERS spectrum observed contains intense bands which are not due to the analyte, but to the competing species. Sometimes, the competing species are already present on the substrate, as impurities or as a "capping" agent, which is intrinsically part of the substrate because of the synthetic method, used for its preparation (e.g the citrate ions present on the surface of the citrate-reduced metal colloids). Thus, observing strong SERS bands in a SERS experiment does not necessarily mean that the bands observed are due to the analyte (Sánchez-Cortés and García-Ramos, 1998). When using metal colloids as substrates, a common mistake is to interpret the intense bands due to citrate as those of the analyte. A direct comparison between the normal Raman spectrum of the analyte and the SERS spectrum obtained must be always done to ensure that the signal observed is actually due to the analyte, and not to interfering species. Impurities can also 
originate spurious SERS bands, as well as amorphous carbon which might form as a consequence of sample photodegradation (Sánchez-Cortés and García-Ramos, 1998).

\section{Photo-induced thermal degradation}

Thermal degradation of adsorbed species as a consequence of intense illumination is, in fact, another common problem for SERS, which often leads to an intense background due to two very broad bands around 1300 and $1600 \mathrm{~cm}-1$, a distinct marker for the presence of amorphous carbon. In SERS experiments, a good practice is to look for these bands and, in case, to decrease the laser power until no bands to amorphous carbon are detected.

\section{Normal Raman and fluorescence: competing processes}

Raman and fluorescence are two processes which are also competing with SERS. Fluorescence is often responsible for intense, sloping backgrounds underlying SERS bands. In worst cases, fluorescence (from the analyte or from impurities) can completely submerge the SERS bands, and no SERS spectrum is observed. Usually, however, excitation in the nearinfrared will minimize the interference from fluorescence, making the observation of SERS spectra possible from otherwise fluorescent samples. Sometimes, normal Raman bands from solvents or concentrated interfering species will also contribute to the spectrum. For instance, analyte solutions containing fractions of solvents such as methanol, ethanol or DMSO, often used to prepare solutions of poorly soluble analytes, might display normal Raman bands of these substances beside the SERS bands of the adsorbed analyte. This is one more reason why it is always advisable to check a spectrum of the "blank" sample (a solution with no analyte present) to get an idea of which bands are due to the matrix itself rather than to the analyte.

\subsection{SERRS: RESONANCE EFFECTS}

Many analytes are "colored", i.e. they present electronic transitions in the visible or near-infrared region, such as the pipi or n-pi transitions for organic molecules having extended systems of conjugated double bonds. When such analytes are probed with a laser having a wavelength corresponding to an energy similar to one of their electronic transitions, absorption processes occur, and the probability associated with the transitions involved in the Raman process is greatly enhanced. In that case, the intensity of the Raman bands is enhanced, and the overall resonant Raman effect is exploited as an enhancement mechanism in what is called "resonance Raman spectroscopy" or RR spectroscopy (Smith and Dent, 2013). This effect can take place even when resonant analytes are adsorbed on nanostructured metal surfaces in the course of a SERS experiment, yielding very intense spectra that benefit from the synergistic combination of both SERS and RR effects. When this is the case, the term used is "double R" SERRS, i.e. surface-enhanced resonance Raman spectroscopy 
(McNay et al., 2011; Smith and Dent, 2013). Because of this RR effect, one always has to keep in mind that the choice of the excitation wavelength, and consequently of the nature of the metal substrate, has to take into account resonant transitions. In other words, if your analyte is "colored", different excitation wavelengths can lead to very different results, i.e. SERS or SERRS, depending if the RR effect is present or not. Usually, the combination of SERS and RR effects in SERRS yield spectra so intense (detection of single molecules SERRS spectra have been repeatedly and consistently reported) that this is purposely exploited to boost the sensitivity of the method. Sometimes, however, SERRS bands due to resonant impurities present in the sample might interfere with the detection of the analyte bands. In any case, one has to remember that a wanted or unwanted RR effect might greatly affect SERS experiment, and thus the choice of a proper excitation wavelength is of utmost importance.

\subsection{ENHANCEMENT FACTORS}

The term "enhancement factor" (EF), in the context of SERS, is a multi-faceted and often misunderstood (and misused) word. Several different definitions of EF have been proposed, creating some confusion (Le Ru et al., 2007). In fact, it is a concept created to quantify with a number how much the signal observed in a SERS experiment is enhanced with respect to a normal Raman experiment. Often, this number is meant to quantify "how good" a SERS substrate is, compared to other substrates. However, things are complicated by the fact that the intensity of SERS signal depend also on the analyte and on the laser used (to name the two most important factors), so that the EF cannot refer to the substrate itself, but to the substrate-laser-analyte combination used.

A general definition for the enhancement factor, which assumes that two experiments (i.e. a SERS one and a normal Raman one) are performed with the same analyte, is

$$
E F=\frac{I_{S E R S} / N_{\text {Surf }}}{I_{R S} / N_{V o l}}
$$

Where $\mathrm{I}_{\mathrm{SERS}}$ is the intensity of the SERS signal and $\mathrm{N}_{\text {Surf }}$ is the number of molecules adsorbed on the metal surface of the SERS substrate in the SERS experiment; $I_{R S}$ is the intensity of the normal Raman signal and $N_{V o l}$ is the average number of molecules in the scattering volume for the normal Raman experiment (Le Ru et al., 2007). This general definition, however, presents some difficulties. While the term $\mathrm{N}_{\mathrm{vol}}$ can be calculated as the product of the molar concentration for the volume probed by the laser and whose signal is collected by the collection optics, the term $\mathrm{N}_{\text {Surf }}$ is much more difficult to estimate, as it depends on the affinity of the analyte for the surface. Moreover, this definition assumes that all the molecules adsorbed on the surfaces are equally contributing to the SERS signal, which is not true in general. A much more viable definition is that of the analytical enhancement factor (AEF), as

$$
A E F=\frac{I_{S E R S} / c_{S E R S}}{I_{R S} / c_{R S}}
$$


Where $c_{S E R S}$ and $c_{R S}$ are the analytical molar concentrations of the analyte in the SERS and normal Raman experiments, respectively (Le Ru et al., 2007). The AEF can be readily calculated, enabling a comparison between different substrates, if the same analyte is used. However, it should be stressed that, since the SERS signal is depending on how a specific analyte is interacting with the surface, the results obtained with one analyte might not hold true for others. In other words, while one substrate is better than a second one in enhancing the signal of an analyte, the reverse could be true when a different analyte is used. Thus, the information given by EFs should be used with care: EFs are useful to compare the performance of different substrates on the same analyte, but extending their use farther than that might be dangerous.

\section{SERS SUBSTRATES: CLASSIFICATION AND GENERAL CHARACTERISTICS}

The availability of nanostructured metal substrates with adequate plasmonic properties is central to SERS. Since the beginnings of SERS on electrochemically or chemically roughened electrodes, many other substrates have been proposed and used. Metal colloids were one of the first substrates to be used besides roughened electrodes, and rapidly became popular because of their ease of preparation and use, and they are still much used today. With the development of nanofabrication techniques and of wet nanotechnology synthetic protocols, a broad variety of SERS substrates have been prepared, so that the literature about this topic is ever growing, and in recent years many commercial substrates became available as well. Given the wide variety of approaches and characteristics, there are many ways in which SERS substrates can be categorized, besides the obvious criterion of the nature of the metal itself.

A very general criterion is to roughly divide the substrates into colloidal and non-colloidal, where the first are constituted by all those substrates made of metal nanoparticles dispersed into a liquid medium, forming a colloid. A problem with this criterion is that the class of non-colloidal substrates (sometimes referred to as "solid substrates") is very heterogeneous.

Another general criterion would be about the nature of the synthetic method used to prepare the substrates: chemical methods (e.g. chemical etching, electrochemical roughening, wet synthesis of nanoparticles by reduction of metallic salts, etc.) or physical methods (e.g. metal sputtering, electron beam nanolithography, nano-imprinting, laser ablation, etc.). However, in many cases a combination of the two approaches is used, so that this criterion is not very efficient.

A third general criterion would be, from the perspective of nanotechnology, the "direction" of the substrate preparation: top-down or bottom-up. Bottom-up substrates would be the ones prepared using already available building blocks are assembled as elements to form the final nanostructure. An example of bottom-up SERS substrate would be a solid substrate whose surface is constituted by self-assembled metal nanoparticles. Top-down substrates, on the other hand, are the ones prepared starting from a bulk material and forming the nanostructure by "sculpting" it, taking away the parts in excess or shaping it so that what is left in the end is the desired nanostructure. An example of a top-down SERS substrate 
would be a nanostructured surface obtained by selectively etching parts of the original surface thanks to nano-lithographic processes.

A fourth criterion, applicable to non-colloidal substrates, is concerning the "regularity" of the metal nanostructures. Such surface structures can be regularly spaced and ordered, such a regular array of nanoholes or nanodomes, or they can be randomly spaces, irregular and disordered.

\author{
*** Insert Figure $2 * * *$ \\ Caption: General classification of SERS substrates \\ Credit: none (original unpublished figure)
}

None of the criteria above, or any other possible criterion (for instance the chemical or physical characteristics of the metal surface), is generally accepted as a "universal" criterion capable of categorizing completely and unmistakably the vast universe of proposed SERS substrates. However, with respect to biomedical applications in particular, where samples are often biofluids, cells or tissues, it might be useful to combine two of the above criteria, first roughly dividing the substrates into colloidal and non-colloidal, and then further sub-classify the non-colloidal substrates as bottom-up or topdown (Figure 2). This categorization is proposed for practical purposes, that is, keeping in mind applications. In fact, samples such as biofluids or cells, for instance, behave very differently when put together with colloidal or non-colloidal substrates for SERS analysis, so that it makes sense to use this criterion when describing the use of different types of SERS substrates.

\title{
2.1. COLLOIDAL SUBSTRATES
}

Because of their simple and straightforward synthetic protocols, low costs of reagents needed, the use of basic, inexpensive laboratory equipment and most important, their effectiveness in enhancing the Raman signal, metal colloids have been and still are widely used as SERS substrates. Although metal colloids made of various transition metals have been reported as SERS substrates, the most used metal colloids are those made of Ag and Au. Different preparation protocols lead to differences in shape and size of the nanoparticles obtained, with a broad variety of morphologies (e.g. nanospheres, nanostars, nanocubes, nanorods, nanoflakes, nano-hollow spheres, etc.) and sizes, from tens to hundreds of nanometers. The shape and size of the nanoparticles, beside the nature of the metal, define the plasmonic characteristics, so that surface plasmons can be tuned to match the exciting laser to be used. For instance, for spherical nanoparticles, the larger the size, the smaller the frequency of the surface plasmons (Amendola and Meneghetti, 2009a; Haiss et al., 2007). The size of nanoparticles is usually a factor to take into account when planning a SERS experiment. The concept of size 
is easy to apply only when considering spherical nanoparticles or nanoparticles having a regular, symmetric shape (e.g. nano-cubes, nano-octaedra, etc.), whereas the description of more complex shapes such as nanorods or nanoplates require the specification of different sizes along different nanoparticle axes. The general concept is that a minimum size is necessary to generate a significant SERS effect, so that nanoparticles of few nanometers will not show any enhancement (Hong and Li, 2013; Njoki et al., 2007; Stamplecoskie et al., 2011). For spherical Au nanoparticles, a SERS effect is reported only when using nanoparticles of at least 30-40 nm of diameter (Njoki et al., 2007). For Ag nanoparticles, the correlation is less well defined, but the fact that the SERS signal depends on the nanoparticle size is established (Stamplecoskie et al., 2011).

Anisotropic shapes often have more than one plasmonic frequency, such as nanorods (J. Orendorff et al., 2006) or nanostars (Guerrero-Martínez et al., 2011; Khoury and Vo-Dinh, 2008). The literature is richer in protocols for the preparation of Au colloids than for Ag colloids, and the shape and size of Au nanoparticles can be better controlled than that of Ag nanoparticles, for which the choice is still somewhat limited. In spite of this wide range of choices, however, for most applications just few of these recipes (or their variants) are used. For most applications involving the direct detection of analytes in aqueous solutions, quasi-spherical nanoparticles dispersed in an aqueous medium are mostly used. $\mathrm{Ag}$ and $\mathrm{Au}$ nanoparticles obtained by reduction of metal ions in $\mathrm{AgNO}_{3}$ or $\mathrm{HAuCl}_{4}$ (or $\mathrm{AuCl}_{4}^{-}$) with citrate ions (in brief: citrate-reduced Ag and Au colloids), have been among the first SERS substrates to be used, and are still widely used (Kimling et al., 2006; Lee and Meisel, 1982). They have the advantage of being simple one-step synthetic protocols in aqueous environment, carried under mild experimental conditions using a readily available apparatus. Moreover, the obtained colloids are rather stable, if kept in the dark at room temperature, and can be stored for months without losing their function as SERS substrates. The colloidal stability is due to the layer of adsorbed citrate ions, conferring a negative charge (well below $-30 \mathrm{mV}$ ) to the nanoparticles surface that hinder aggregation thanks to the inter-particle electrostatic repulsion. With the citrate-reduction method, it is possible to obtain spheroidal Au nanoparticles having well-defined sizes (Njoki et al., 2007). A strong correlation between particle size and the maximum of the extinction band has been reported, so that an indication of the size can be simply obtained from an extinction spectrum. A rather different situation is encountered in the case of citrate-reduced Ag nanoparticles (Lee and Meisel, 1982), for which nanoparticles with a broad range of shapes (mainly spheroids, rods, plates) and sizes are obtained.

Another widely used protocol for obtaining Ag colloids is using hydroxylamine hydrochloride as reducing agent (Leopold and Lendl, 2003), leading to spherical Ag nanoparticles of sizes ranging from 23 to $67 \mathrm{~nm}$, depending on the ratio between the reagents. In that case the surface of the nanoparticles is also negatively charged, but because of the presence of adsorbed chloride ions. Limiting the adsorbates on the nanoparticles surface to simple atomic ions promotes the adsorption of analytes, which do not need to displace adsorbed molecular species, such as citrate ions or other capping 
agents. Metal colloids prepared by laser ablation (Amendola and Meneghetti, 2009b) also present the advantage of having a "naked" surface, devoid of molecular adsorbates.

In general, different synthetic protocols lead to nanoparticle surfaces with different physical and chemical characteristics. Often, the production of nanoparticles with more complex shapes requires the use of selective capping agents binding onto specific crystal facets to control the direction of crystal growth, and such capping agents need to be used in organic solvents and are difficult to remove, hindering the adsorption of the analyte on the metal surface, and interfering with the SERS analysis. Problems related to the use of capping agents binding too strongly to the metal and to the need of organic solvents (usually interfering because of their own intense Raman spectrum) as dispersing medium are limiting the use of many metal colloids other than the simple, quasi spherical metallic nanoparticles dispersed in aqueous media.

In spite of the wide choice of colloidal syntheses available, no protocol or shape is accepted as "standard", and in absence of standards each lab use its own recipe. This lack of standardization, together with the well-known repeatability issues linked to colloidal synthesis, makes a direct comparison of results obtained by different labs problematic, and constitutes a serious obstacle to the development of SERS as a standard analytical technique to be used outside academia. Moreover, the most efficient plasmonic nanostructures obtained from metal colloids are the nanoparticles aggregates, which help the formation of nano-sized gaps between particles (called hot-spots (L. Kleinman et al., 2013)) where the electromagnetic field amplification as required by the EM is particularly intense. Although still debated, evidences are supporting the fact that the SERS effect from single spherical metal nanoparticles is negligible with respect to that of aggregates (Zhang et al., 2015). The situation is more complicated for anisotropic nanoparticles such as nanostars or nanorods, for which it seems that SERS from single nanoparticles, especially from those molecules adsorbed on specific nanoparticle locations, is comparable to that of aggregates (Guerrero-Martínez et al., 2011). Still, at least in the case of spherical nanoparticles, which are the most commonly used, aggregation is needed to get a significant SERS effect. Spontaneous aggregation can be induced upon addition of the analyte solution, for different reasons. The analyte itself might readily adsorb in large amounts onto the nanoparticles surface, causing a sudden decrease of surface charge leading to the destabilization of the colloid, since the electrostatic repulsion between different nanoparticles is not enough to keep them apart anymore.

In the case of citrate-reduced colloids, for instance, since citrate ions are already present on the nanoparticles surface, a necessary condition to observe a SERS signal from an analyte is that it must be able to displace the citrate from the surface by strongly adsorbing on the metal. As a consequence, a major limitation of citrate-reduced metal colloids as SERS substrates is that their use with analytes bearing a net negative charge is problematic because of the analyte-particle repulsion. The addition of positively charged polyelectrolytes (e.g. poly-amines) to the system usually helps in mediating the interaction between those analytes and the negatively charged colloids, working as an "electrostatic glue" between the two (Garcia-Rico et al., 2018; Marsich et al., 2012). 
Ionic species, if present in the analyte solution, can shield electrostatic interactions, including those causing the repulsion between the colloidal particles, eventually leading to aggregation. However, it might be that the analyte is too diluted, and that the ionic strength of the solution is too low to induce a spontaneous aggregation, in which case some electrolytes (e.g. salts, acids, bases) can be purposely added to the system to induce aggregation.

Because of the electrostatic nature of the stability of the citrate-reduced colloids, for instance, these can be easily aggregated to maximize the SERS effect by increasing the ionic strength upon the addition of salts or saline solutions. There are some circumstances, however, in which aggregation is hindered, e.g. by the presence of species which sterically stabilize the colloid, such as thick polymer coatings or layers of proteins around the nanoparticles (Gebauer et al., 2012; Ho et al., 2018). In those cases, the need for aggregation limits the use of most common colloidal substrates. Often, to overcome problems related to aggregation, colloidal substrates are pre-aggregated (by adding small quantities of an electrolyte) before the addition of the analyte solution. In that case, small nanoclusters, or even dimers or trimers of nanoparticles are formed, forming the plasmonic nano-gaps before coming in contact with the analyte solution.

\subsection{BOTTOM-UP NON-COLLOIDAL SUBSTRATES}

Metal nanoparticles obtained by various protocols can be then assembled onto solid substrates, to form nanostructured surfaces which can be used as SERS substrates. Solid substrates used can be "hard" and compact solids, such as silicon, quartz or glass, or "soft" or porous such as polymers or paper. Simple, readily available and inexpensive substrates as glass and paper are often used. In particular, paper-based substrates (Figure 3) are raising an increasing interest: they are flexible, inexpensive, porous and allow the integration of chromatographic or microfluidics approaches to pre-process the sample before SERS analysis (Dalla Marta et al., 2017; F. Betz et al., 2014; Hoppmann et al., 2013; Restaino and White, 2019). The nanoparticles dispersed in the colloid can be assembled onto the solid substrates by different methods, such as jet printing, spraying, drop casting or dipping, usually leaving nanoparticles to self-assemble in random aggregates once the liquid medium evaporates. Sometimes, the nanoparticles are created directly on a solid substrate ("in situ nanoparticle synthesis") (Virga et al., 2013). In all these cases, nanoparticles usually form irregular, disordered nanostructures, and "hots-spots" are irregularly distributed.

Credit: (Dalla Marta et al., 2017) 
Another method that can be considered as "bottom-up" is nano-sphere lithography (Hulteen and Van Duyne, 1995). In this method, polystyrene or $\mathrm{SiO} 2$ nano-spheres are deposited on a solid substrate (e.g. glass or silicon), forming ordered monolayers in which the spheres are regularly packed. Then, an Au or Ag layer is deposited on the top of these spheres (Figure 4). The surface obtained is called Ag-FON (film over nano-spheres), and its plasmonic properties make it an excellent SERS substrate. Alternatively, the spheres can be removed, leaving regularly-spaced triangular metal nanoparticles where the interstitial spaces of the nano-spheres layer were.

$* * *$ Insert Figure $4 * * *$

Caption: Schematic illustration of a process to create a SERS substrate (AgFON: Film Over Nanospheres) with nanospheres lithography.

Credit: none (original unpublished figure)

In general, the preparation of bottom-up non-colloidal substrates does not require special or particularly expensive instrumentation, while giving SERS substrates with good performances that can be used for in-vitro diagnostic applications (see section 3.3).

\subsection{TOP-DOWN NON-COLLOIDAL SUBSTRATES}

Top-down substrates can be approximately divided in two classes: substrates with ordered, regular surface structures and substrates with disordered, irregular, randomly arranged structures. Chemical etching of metal plates with strong acids is perhaps the simplest method to obtain roughened metal surfaces having irregular nanostructures. However, this method is not very reproducible and yields SERS substrates which are not very efficient. Another "chemical" method , which however requires the availability of an electrochemical setup, is the electrochemical roughening of metal surfaces with oxidation-reduction cycles (ORC) (Roth et al., 1993). This method leads to roughened metal surface with disordered nanostructures. Physical methods such as laser ablation can be also used to create SERS substrates with disordered features (Lee et al., 2001). Irregular surface nanostructures can also be created by physical or chemical etching methods on materials other than metal (e.g. silicon, Figure 5), and then be successively coated with Ag or Au to obtain the plasmonic properties desired for SERS (Schmidt et al., 2012). Ordered metal nanostructures, on the other hand, are usually obtained by using electron-beam lithography techniques (Mosier-Boss, 2017).

$* * *$ Insert Figure $5 * * *$ 
Caption: Scanning electron microscope images of a SERS substrate (i.e. Ag-coated Si nanopillars) prepared with a topdown approach.

Credit: (Schmidt et al., 2012)

\subsection{DESIRABLE CHARACTERISTICS}

Besides an adequate EF, several other characteristics are important when considering the performance of SERS substrates. The most important characteristic for a SERS substrate is perhaps its reproducibility. In SERS, however, "reproducibility" is a complex, multi-fold concept, which is often misunderstood.

The reproducibility of a SERS substrate, for instance, might refer to the fact that spectra obtained on different substrates are qualitatively similar, i.e. they have bands at the same Raman shift and with the same "intensity pattern". That is, their overall intensity can be different, but when normalized they are ideally identical. This kind of reproducibility can be easily assessed by collecting several spectral replicates (i.e. 5-10) of the same sample on different substrates and by evaluating the spectral variability (e.g. the standard deviation of the intensity of one or more bands) after intensity normalization. As an option, this operation can be done also after the subtraction of a baseline. That is the minimum requirement for the reproducibility of a SERS substrate, allowing the development of methods for qualitative analysis or classification (e.g. identification or diagnosis). If an internal standard is used, this reproducibility will actually also allow the development of quantitative methods. This kind of reproducibility should not be given for granted, since even small variations in the preparation protocol for the substrates, which might be due to different operators or to other experimental factors, can lead to small differences in the surface chemistry of the metal substrate, and thus to a slightly different analyte-metal interaction which is will affect the SERS spectra.

An entirely different matter is the reproducibility of the overall intensity of the SERS spectra obtained from different substrates, i.e. the fact that two spectra can be overlaid even without normalization. This kind of reproducibility can be assessed by collecting several replicates of the same sample of different substrates and by calculating the intensity variability (e.g. the intensity standard deviation) of one or more bands, without performing an intensity normalization. Also in this case, this procedure can be also done after the subtraction of a baseline from all spectra, to compensate for differences in the background. The reproducibility in terms of absolute intensity is much more difficult to achieve, and it is very important when using the substrates for quantitative analysis.

Moreover, for non-colloidal substrates it is important to distinguish between intra- and inter-substrate reproducibility. For these substrates, spectra can in fact be collected from different areas or spots of the substrate, so that differences can be observed in spectra collected from different spots. The heterogeneity of a SERS substrate, both in terms of qualitative 
and quantitative response, can be assessed by collecting several replicates from different spots, or mapping an area of the substrates, and by calculating the spectral variability.

There is no general or standard procedure to assess the reproducibility of SERS substrates, but in any case all these aspects, and in particular, in the case of non-colloidal substrates, both the intra- and inter-substrate reproducibility should be considered.

Often, SERS substrates often have adsorbates on their surface prior to the analyte addition. For colloidal substrates, these are the species conferring the colloidal stability (e.g. citrate), whereas for non-colloidal substrates some adsorbates can be present as a consequence of the preparation protocol, or as impurities. Thus, it is not uncommon to have substrates displaying a background signal which might interfere with the bands due to the analyte. For instance, all metal colloids prepared by citrate reduction have citrate ions ad adsorbates, yielding a characteristic SERS spectrum which might interfere with that of the analyte. A "flat" or low background, however, is certainly a desirable characteristic for SERS substrate, as it makes analyte detection easier.

A long shelf-life, that is the capability of retaining its characteristics in time, is also a distinct advantage for a SERS substrate. Another desirable characteristic for SERS substrate, which will also impact on its shelf-life, is the stability toward a wide range of physical (e.g. temperature or light) or chemical (e.g. pH or ionic strength) conditions. Often, measurements must be performed in relatively harsh physical or chemical conditions, such as extremely acidic or basic $\mathrm{pH}$. Citrate-reduced colloidal substrates, for instance, have a long shelf-life (months), but conditions as $\mathrm{pH}$ and ionic strength heavily impact on their stability, sometimes compromising the detection of a SERS spectrum.

Economic aspects such as substrates costs and their re-usability are also important. In the development of many biomedical and diagnostic applications, a considerable number of independent spectra must be collected in order to build statistically significant predictive models. Thus, the costs related to a single measurement cannot be too high, so that producing or buying the necessary substrates is feasible with a reasonable budget. The low costs are one of the reasons why paper-based substrates are gaining popularity, offering a reasonable trade-off between efficiency and cost.

While colloidal substrates can be used just once, non-colloidal substrates, at least in principle, could be "re-generated" to be re-used more than once. Both physical (e.g. plasma cleaning or UV-light) or chemical (e.g. exposure to strongly oxidizing or reducing agents) methods could be used to get rid of the organic matter present on the metal surface, without compromising the nanostructure itself (Negri et al., 2011; Sadate et al., 2010; Siegfried et al., 2013).

\subsection{CHARACTERIZATION TECHNIQUES}

\section{Characterization of colloidal substrates}


The simplest tool to characterize colloidal substrates is UV-visible absorption spectroscopy, yielding so called "extinction spectra", which are the resultant of both scattering and absorption phenomena (Petryayeva and Krull, 2011). Extinction spectra give an indication of which are the plasmonic frequencies of the nanoparticles, but one has to remember that aggregated nanoparticles will behave differently from individual ones, so that the extinction spectra will depend on the aggregation state, with aggregated nanoparticles showing a red-shifted maximum and a much broader band. Thus UVvis spectroscopy is also useful to determine the aggregation state of your system. Moreover, so called "dark-modes" will be not visible from a far-field approach such as a UV-vis absorption experiment (Barrow et al., 2014; Koh et al., 2009), but can play a significant role on the SERS performance of the colloids. For instance, spheroidal Ag nanoparticles display an extinction maximum between $390-420 \mathrm{~nm}$, and, if aggregated the extinction maximum will shift to the green or even to the yellow-orange part of the spectrum. However, such particles will display intense SERS spectra even when excited with a near-infrared laser (e.g. at $785 \mathrm{~nm}$ ), because of the occurrence of dark plasmonic modes around that wavelength (Álvarez-Puebla, 2012).

Extinction spectra can also be used to check the shape of the nanoparticles: depending on the shape, nanoparticles can support more than one plasmonic frequency. For instance, nanorods show two extinction maxima, corresponding to two plasmonic frequencies: one frequency for each axis of the nanoparticles (i.e. short one and long one) (J. Orendorff et al., 2006). Also nanostars show two extinction maxima, one for the "core" and one for the "spikes" of the particle (GuerreroMartínez et al., 2011).

Since extinction spectra depend on the shape and size of the nanoparticles, the width of the extinction band will give a gross indication of the size distribution of the particles. If nanoparticles have only one definite shape (e.g. spherical), then the narrower is the width of the extinction band, the more mono-disperse is the colloid. This is not useful for precise and absolute analysis about size distribution, but rather to qualitatively compare the size distribution different colloid batches. For spherical Au nanoparticles, thanks to some detailed studies (Njoki et al., 2007), it is possible to use UV-visible extinction spectra to get a rather precise estimation of their size and concentration. However, for other shapes or metals, it is still necessary to use other characterization technique to get a more precise estimation of nanoparticle size and concentration.

Although transmission electron microscopy (TEM) is the safest and more accurate method to get information about the size and shape distribution of metal nanoparticles, less expensive and non-destructive methods based on light-particle interaction and Brownian motion analysis such as dynamic light scattering (DLS) or nanoparticle tracking analysis (NTA) (Hole et al., 2013) can be also used. Moreover, zeta-potential measurements, often combined with DLS and NTA are extremely useful. The zeta-potential is actually the potential difference, measured in $\mathrm{V}$ or $\mathrm{mV}$, between the static layer of fluid around the nanoparticle and the bulk medium in which the nanoparticles are dispersed, but it can indirectly give an 
indication about the surface charge of the nanoparticles (Bhattacharjee, 2016). Usually, for electrostatically stabilized colloids (as most of SERS colloidal substrates), absolute values higher than $30 \mathrm{mV}$ are indicative of a stable dispersion. Zeta-potential measurements are also particularly useful for the qualitative determination of the surface charge, especially in those cases in which one aims at reversing this charge (e.g. from negative to positive) by substituting the adsorbed species forming the so called "capping layer".

\section{Characterization of non-colloidal substrates}

In principle, non-colloidal substrates can be characterized with all the methods available in the field of surface science (O’Connor et al., 2013). However, most used methods include scanning electron microscopy (SEM), often coupled with energy-dispersive $\mathrm{x}$-ray spectroscopy (EDS), scanning tunnelling microscopy (STM) and atomic force microscopy (AFM). All of these can be used to get information about the substrate topology on the nanoscale, with SEM giving better results when investigating highly irregular surfaces such as those formed by random deposition of nanoparticles aggregates. EDS is also yielding information about elemental composition of the surface, including information about elements constituting the adsorbates. In EDS spectra, however, all molecular information about the adsorbed species is lost. Secondary ion mass spectrometry (SIMS) can give some more information about adsorbed molecules, since charged molecular fragments are detected.

Plasmonic frequencies arising from opaque, optically dense non-colloidal substrates can be investigated with UV-visNIR diffuse reflectance spectroscopy. This technique works particularly well with highly irregular and porous surfaces, such as those of nanoparticles-on-paper substrates (Weng et al., 2018).

\section{SERS SUBSTRATES FOR BIOANALYSIS, DIAGNOSTICS AND THERANOSTICS}

There is no such thing as a "general" SERS substrate that can be used with any analyte. Since the SERS response is the result of a complex interplay between the analyte, the matrix and the metal substrate, each analytical problem requires a SERS substrate with its own proper characteristics. It is highly advisable to choose the metal nanostructure in function of the analytical problem, and of the overall strategy chosen to tackle it. Biological samples such as biofluids (e.g. plasma, serum, urine and saliva) are chemically complex mixtures, often containing several thousands of chemical species (Bouatra et al., 2013; Psychogios et al., 2011). Sometimes the goal is to obtain a biochemical fingerprint of a biological sample, without specifically targeting one analyte, but aiming to get as much information as possible from SERS spectra, thus hoping to detect as many biomolecules as possible. This is called an "untargeted" approach. In other cases, one is interested in a specific analyte. Detecting or quantifying a specific analyte amidst all the biochemical species constituting the biological matrix, without a separation step involving a chromatographic approach, is a formidable task, requiring a 
definite strategy and, accordingly, a substrate with suitable properties. The SERS substrate must meet at least two requirements: it should have a plasmonic response at the wavelength selected for excitation, and it should be able to capture or bind the analyte of interest.

\subsection{INDIRECT VS. DIRECT SERS DETECTION}

The first and most important aspect to define in order to design or select a suitable substrate is the choice between a direct detection and an indirect detection strategy (Figure 6). A direct detection of the analyte involves the direct sensing of the vibrational bands due to the analyte or analytes of interest, whereas in the indirect detection, the presence or quantity of the analyte or analytes is inferred from the variation in intensity or Raman shifts of bands due to vibrations of other molecules (probes). The main challenge in the direct detection strategy, especially when the matrix in which the specific analyte of interest is found is chemically complex (e.g. a biofluid), is to limit the interference from all the other chemical species, which will compete with the analyte for the adsorption onto the metal surface (see section 3.2). Usually, unless the analyte itself has a very good affinity for the metal surface, the direct detection of a specific analyte in a complex matrix is very challenging. Lowering the complexity of the matrix (e.g. by introducing some pre-processing steps such as analyte extraction) or modifying the surface to make it more attractive for the analyte are two possible option. Strategies for surface functionalization include the modification of the surface charge or hydrophobicity, for instance using selfassembled monolayers (SAM). Alternatively, the analyte can be forced to bind close to the surface by a chemical reaction causing the formation of a bond between the analyte and a small molecule immobilized on the metal surface. In any case, the surface functionalization should not increase too much the distance between the analyte and the surface, otherwise the SERS effect, which rapidly decreases with the distance from the metal, will be negligible. If the surface has physicochemical characteristics (e.g. surface charge) which are compatible with the analyte of interest, the direct detection usually does not require further substrate functionalization. The direct detection strategy, since it is carried out without the use of Raman reporters or labels, is usually referred to as "label-free". An "untargeted" label-free approach is also possible, so to retain as much as possible of the biochemical complexity of the sample. In this approach, one does not look for a specific analyte but for as many biomolecules (e.g. metabolites) as possible, so that the interference from the matrix becomes a lesser problem.

$* * *$ Insert Figure $6 * * *$

Caption: An example of indirect (on the left) and direct (on the right) detection strategies for SERS of biofluids using colloidal substrates.

Credit: (Bonifacio et al., 2015) 
In an "indirect detection" approach, on the other hand, substrate functionalization is usually required. Raman reporters or labels are used in order to reveal the presence of the analyte, and they can be used according to different strategies. A common strategy is to bind both Raman reporters and recognition elements (e.g. antibodies) to nanoparticles, obtaining objects that are often called "SERS nanotags" (Laing et al., 2017). These SERS nanotags can be used in tests in which the target analyte is first captured by recognition elements on a different substrate, and then the nanotags, upon binding to the target, are used to reveal if the analyte is present. Another strategy is to use SERS beacons, i.e. molecular systems that can vary the distance (increasing it or decreasing it) between a Raman reporter and the metal surface in presence of the analyte (Wei et al., 2013). A different approach consists in using chemical reactions between a Raman reporter bound on the surface and the analyte, while looking for changes in the signal of the reporter (Sharma et al., 2016; Sun et al., 2014). Often, Raman reporters are dyes in resonance with the exciting laser, so that the further enhancement given by SERRS can be exploited (Graham and Faulds, 2008; Sabatté et al., 2008).

Glucose as an example where both direct and indirect approaches have been tried. The direct approach has been challenging, since glucose has little affinity for gold or silver surfaces, so that metal surfaces must be functionalized with self-assembled monolayers capable to bind the sugar (Lyandres et al., 2008; Yonzon et al., 2006). In the indirect approach, glucose was captured by organoborates via a chemical reaction, causing changes in the spectrum of these molecules that were clearly detectable, allowing the indirect sensing of glucose even in biological media (Sharma et al., 2016; Sun et al., 2014). These results were obtained using non-colloidal substrates, which allowed a better surface functionalization and avoided problems related to interference from other molecules present in the biological media.

\subsection{THE ROLE OF THE NANO-BIO INTERFACE}

When considering bioanalytical SERS applications using biological samples, one has to carefully consider the biochemical complexity of the biological matrix. When a nanostructured metal substrate is put in contact with a biological sample such as a biofluid, many biomolecules will spontaneously adsorb on the metal surface, creating a complex system called nano-bio interface (Nel et al., 2009). The nano-bio interface has been well characterized in the case of gold nanoparticles and blood or cells, especially as far as proteins are concerned (Docter et al., 2015; Piella et al., 2017), but information about such interface in the case of other nanostructured gold and silver surfaces or other biological samples is rather limited. What is know from Au nanoparticles, is that as soon as these nanostructures enter in contact with a protein-rich biological environment, such as blood or cytoplasm, a layer of adsorbed proteins called "protein corona" rapidly forms (Docter et al., 2015). A similar layer also forms on non-colloidal metal surfaces, which, depending on the specific application, may impair their function as SERS substrates (“protein fouling”) (Blaszykowski et al., 2012). Besides 
protein, a plethora of small molecular weight biomolecules can strongly adsorb on the metal surface, possibly interfering with the SERS detection of an analyte. In general, when looking for a specific analyte with SERS, the formation of a nano-biointerface can cause two type of problems. First, it can saturate the substrate surface, impeding the adsorption of the analyte on the metal surface. Second, even if the analyte has an affinity and concentration allowing it to co-adsorb together with the matrix biomolecules, the signal of the latter may strongly interfere with the bands due to the analyte, de facto hindering its detection, especially in the case of a direct detection strategy. This is true for all kinds of SERS substrates, but for colloidal substrates, there is another major problem with the formation of a protein corona: the hindering of colloidal aggregation by steric stabilization of the nanoparticles. Since colloidal aggregation is functional for the formation of SERS active sites, biological samples with a high protein concentration (e.g. blood serum or plasma), by promptly forming a protein corona around nanoparticles, may yield weak SERS spectra, or no SERS spectra at all. Thus, for protein-rich samples a de-proteinization step (e.g. by filtration) is often required to obtain intense SERS spectra from colloidal substrates. A pre-aggregation step, by addition of an aggregating agent or by increasing nanoparticles concentration by centrifugation, is also an option to overcome the problems caused by the protein-corona (Bonifacio et al., 2014).

Protein-corona and protein-fouling however, is only part of the problem, and it can solved by methods such as deproteinization or, in the case of non-colloidal substrates, by functionalization of the surface with an anti-fouling coating which allows the detection of the analyte. From SERS data, we know that in many cases (e.g. plasma, serum and cytosol) low-molecular weight molecules are strongly adsorbing on the metal surface, forming a "small-molecules corona" (Bonifacio et al., 2015, 2014; Genova et al., 2018; Hassoun et al., 2017). These molecules, mostly purines and -SH containing molecules (e.g glutathione) can saturate the available sites on the metal surface, of can yield such strong SERS signal to obscure the signal due to the analyte, especially when a direct detection strategy is employed. Moreover, the variability of the biological matrix signal (e.g the inter-individual variability in the case of blood or urine samples) is often making a univariate data analysis, where the intensity or area of a single band is considered, unfeasible, in favour of a multivariate approach. A possible solution is to functionalize the metal surfaces with a layer having the two-fold function of protecting the surface against the unwanted adsorption of small-molecules of the matrix and of promoting the adsorption of the analyte (Sun et al., 2016). Such a functionalization is not trivial: among others, the use of molecularly imprinted polymers (MIP) has been suggested (Bompart et al., 2010; Kostrewa et al., 2003) as a possible strategy.

\subsection{SERS SUBSTRATES FOR IN VITRO DIAGNOSTICS}

Both colloidal and non-colloidal SERS substrates can be used for in vitro diagnostics, with both direct and indirect detection strategies. Samples such as biofluids, especially serum or plasma, which are rich in proteins, might constitute a 
problem for analytical strategies using colloidal substrates and requiring aggregation (see section 3.4), while non-colloidal substrates might incur in the problem of protein fouling. Biofluids can be directly deposited on non-colloidal substrates, but then they must be left to incubate for some time and washed away, or let dry. In the latter case, depending on the drying conditions, the sampling area can become extremely heterogeneous, with different parts of the substrate yielding different spectra, to the detriment of experiment repeatability. Moreover, depending on the volume of biofluid, the drying process can take some time, from 15-30 min (for few microliters) to more than 1 hour. On the other hand, colloidal substrates require a "mixing" step with the biofluid sample, but then the resulting mixture can be immediately measured without delays. SERS substrates, in a point-of-care (POC) perspective, can also be incorporated into lateral flow assays devices (Gao et al., 2017; Marks et al., 2017; Tran et al., 2019), so that sample pre-processing or separation steps can be performed on the sample before it reaches the substrate (Figure 7). Colloidal substrates deposited or ink-jet printed on paper can also be part of a so-called "paper analytical device" (PAD) (Abbas et al., 2013). These devices are single-use analytical platforms on small pieces of paper, onto which polymers or waxes are printed to design microfluidic channels for separation, mixing or other pre-processing steps for the sample before SERS detection. These paper-based SERS devices are particularly attractive for diagnostic applications in a clinical setting or even in a POC perspective, since they are affordable, robust and easy to manage and use.

$* * *$ Insert Figure $7 * * *$

Caption: Schematic illustration of the operation principle of SERS paper-based lateral flow strip (PLFS). (a) Top and side views; (b) side view before and after biomarker detection; (c) optical photos of PLFS assembled in cassettes in the presence (upper) and absence (bottom) of the target.

Credit: (Gao et al., 2017)

If the in-vitro diagnostic test has to be performed on cells, the type of substrate to be used depends on the analytical strategy used. Usually, SERS nano-tags labelled with a Raman reporter are used to detect specific cells with an indirect detection strategy, as in the case of circulating tumor cells (CTCs) (Wang et al., 2011; Wu et al., 2015). These tags are meant to bind the external cell membrane of specific cells, revealing their presence in the sample. More recently, other approaches to characterize cells were proposed, such as the analysis of cell lysates (Genova et al., 2018; Hassoun et al., 2017) using colloidal substrates, or the analysis of the cell secretions (also called SERS optophysiology) using noncolloidal substrates (Lussier et al., 2016). These two approaches, however, have been proposed as methods to characterize cells and still need to be tested as diagnostic methods. 
The use of metal nanoparticles as label-free sensors inside intact cells (i.e. after an active or passive uptake) is also possible (Altunbek et al., 2016; Kneipp et al., 2007; Kneipp and Drescher, 2014; Taylor et al., 2016), but results reported by different studies are rather heterogeneous and no diagnostic applications have been reported yet.

\subsection{SERS SUBSTRATES FOR IN VIVO DIAGNOSTICS AND THERANOSTICS}

The type and characteristics of a SERS substrate to be used in vivo must be selected according to its purpose: SERS can be used for the direct sensing of a specific analyte (e.g. glucose levels in the blood) or for the disease detection in terms of spatial localization. Usually, the latter is achieved by using SERS nano-tags to define where the diseased tissue is spatially located. In this sense, the intense signal due to SERS nano-tags is used as a contrast agent for imaging. This approach can be used in diagnostics, to detect and locate the diseased tissue in the body, using a spatially offset approach (Stone et al., 2011) for regions relatively close to the body surface or coupled to endoscopy (Zavaleta et al., 2013) to reach inner tissues. The same approach can even be used intraoperatively to guide the surgeon in defining the margins on the diseased tissue to remove (Jiang et al., 2019). In all these cases, the design of the SERS nano-tag is guided by the same principles (Figure 8), and the choices to be made strictly depend on the final application.

$* * *$ Insert Figure $8 * * *$

Caption: Schematic illustration of the elements constituting a SERS nano-tag.

Credit: none (original unpublished figure)

$\mathrm{Au}$ is mostly used as metal for SERS nano-tags to be used in-vivo, because of its lower chemical toxicity with respect to other SERS metals (e.g. Ag or $\mathrm{Cu}$ ). Bulk Au is chemically inert and a-toxic. Still, nanomaterials such as nanoparticles can display a toxicity related to the size and shape of the material rather than on its chemical composition. This aspect is still being investigated for Au nanoparticles, so that toxicity still remains a concern (Laing et al., 2017). For this reasons, diagnostic approaches based on SERS using topically applied nanoparticles (e.g. in combination with endoscopy (Wang et al., 2015, 2014)) are considered safer with respect to those requiring intra-venous administration of nanoparticles.

The morphology of the nanoparticles is important in defining its plasmonic properties, and thus which laser can be used to get a SERS effect. In general, biological tissues are more "transparent" in a spectral region going from the red to the near-infrared (Lane et al., 2018), so Au nanoparticles with morphologies such as nano-starts, nano-rods or hollow nanospheres, having extinction maxima in those regions, are preferred. Moreover, these nanoparticles can efficiently convert the absorbed light into heat, making them ideal candidates for photo-thermal therapy applications combined with 
diagnosis (i.e. theranostics) (Gao et al., 2015; Lu et al., 2010; Maltzahn et al., 2009; Rycenga et al., 2009; Vo-Dinh et al., 2013).

Raman reporters to be embedded in SERS nanotags for in-vivo applications must give a signal as strong as possible: ideally, dyes absorbing in the NIR should be used (Lane et al., 2018), so to exploit a SERRS effect (see section 1.4), maximizing the signal intensity. To prevent the release of these potentially toxic Raman reporters into the organism, as well as to protect them from unwanted accidental desorption due to potentially aggressive biological environments, a protective coating layer, made of polymers, proteins or silica, is used (Laing et al., 2017).

The SERS nanotags must reach and accumulate in the diseased tissue via passive or active mechanisms. Nanoparticles can passively accumulate in the diseased tissues, but most often active targeting strategy is to be preferred, by functionalizing the SERS nano-tags surface with specific targeting elements such as antibodies, folic acid or aptamers (Laing et al., 2017).

When the purpose of in-vivo SERS sensing is the detection of a specific analyte (e.g. glucose), different strategies are employed, involving the use of non-colloidal substrates. Implanted solid SERS substrates (Ma et al., 2011; Stuart et al., 2006), patches with intradermal micro-needles (Kolluru et al., 2019; Yuen and Liu, 2014) or macroscopic needles with a nanostructured tip or surface (Dong et al., 2012, 2011; P. Li et al., 2017) have been used for this purpose. In these cases, toxicity is no longer a major concern, whereas the challenge is to keep the substrate "active" for a longer time, preventing its degradation due to the interaction with the biological environment and/or the irreversible saturation of its sensing surface with the analyte. A proper surface functionalization, by protecting the metal surface while ensuring a reversible analyte trapping, can play a crucial role in solving these problems (Laing et al., 2017), but, as in other SERS applications, there is still no general solution, and each analytical problem must be specifically addressed.

\section{CONCLUDING REMARKS AND PERSPECTIVES}

SERS substrates are complex objects addressing a complex function, and their design necessarily require an interdisciplinary expertise. Plasmonic aspects have to be considered according to specific physical models; surface functionalization requires a careful chemistry, and the coating with targeting molecules or recognizing elements involves a biological knowledge of the disease involved. In this sense, designing a SERS substrate for bioanalysis perfectly embodies the intrinsic multidisciplinary nature of nanotechnology.

Because of their complexity, SERS substrates must be tailored to the specific bioanalytical problem: experimental details such as the wavelength of the laser to be used, apparently less relevant, are extremely important in defining many aspect of the substrate, so that nothing should be left to the chance. 
Perhaps the most important decision to be made when planning the development of a SERS substrate for bioanalytical purposes is its final use: will it be used in-vivo or in-vitro? For an in-vivo substrates, the options are limited, whereas the in-vitro detection allows for a broader variety of choices. Then, another crucial decision is the strategy to be adopted: direct versus indirect detection. This decision will have consequences over all the other aspects, from the nature of the metal to be used (and then, as a consequence, the type of laser to be used) down to the complexity of the surface functionalization. In all cases, the interplay between the nanostructured metal surface and the incredibly complex and rich biological environments, be that of biofluids, of tissues or of cells, must be reckoned with. To summarize: selecting or designing a SERS substrate for diagnostic or theranostic purposes is far from trivial, and it is a task requiring a considerable amount of effort, including a careful planning about the strategy to be used.

\section{REFERENCES}

Abbas, A., Brimer, A., Slocik, J.M., Tian, L., Naik, R.R., Singamaneni, S., 2013. Multifunctional Analytical Platform on a Paper Strip: Separation, Preconcentration, and Subattomolar Detection. Anal. Chem. 85, 39773983. https://doi.org/10.1021/ac303567g

Altunbek, M., Kuku, G., Culha, M., 2016. Gold Nanoparticles in Single-Cell Analysis for Surface Enhanced Raman Scattering. Molecules 21, 1617. https://doi.org/10.3390/molecules21121617

Álvarez-Puebla, R.A., 2012. Effects of the Excitation Wavelength on the SERS Spectrum. The Journal of Physical Chemistry Letters 3, 857-866. https://doi.org/10.1021/jz201625j

Amendola, V., Meneghetti, M., 2009a. Size Evaluation of Gold Nanoparticles by UV-vis Spectroscopy. J. Phys. Chem. C 113, 4277-4285. https://doi.org/10.1021/jp8082425

Amendola, V., Meneghetti, M., 2009b. Laser ablation synthesis in solution and size manipulation of noble metal nanoparticles. Physical Chemistry Chemical Physics 11, 3805-3821. https://doi.org/10.1039/B900654K Aroca, R., 2006. Surface-Enhanced Vibrational Spectroscopy. John Wiley \& Sons, Chichester.

Barrow, S.J., Rossouw, D., Funston, A.M., Botton, G.A., Mulvaney, P., 2014. Mapping Bright and Dark Modes in Gold Nanoparticle Chains using Electron Energy Loss Spectroscopy. Nano Lett. 14, 3799-3808. https://doi.org/10.1021/nl5009053

Bhattacharjee, S., 2016. DLS and zeta potential - What they are and what they are not? Journal of Controlled Release 235, 337-351. https://doi.org/10.1016/j.jconrel.2016.06.017

Blaszykowski, C., Sheikh, S., Thompson, M., 2012. Surface chemistry to minimize fouling from blood-based fluids. Chemical Society Reviews 41, 5599-5612. https://doi.org/10.1039/C2CS35170F

Bompart, M., Wilde, Y.D., Haupt, K., 2010. Chemical Nanosensors Based on Composite Molecularly Imprinted Polymer Particles and Surface-Enhanced Raman Scattering. Advanced Materials 22, 2343-2348. https://doi.org/10.1002/adma.200904442

Bonifacio, A., Cervo, S., Sergo, V., 2015. Label-free surface-enhanced Raman spectroscopy of biofluids: fundamental aspects and diagnostic applications. Anal Bioanal Chem 407, 8265-8277. https://doi.org/10.1007/s00216-015-8697-z 
Bonifacio, A., Dalla Marta, S., Spizzo, R., Cervo, S., Steffan, A., Colombatti, A., Sergo, V., 2014. Surfaceenhanced Raman spectroscopy of blood plasma and serum using $\mathrm{Ag}$ and $\mathrm{Au}$ nanoparticles: a systematic study. Anal Bioanal Chem 406, 2355-2365. https://doi.org/10.1007/s00216-014-7622-1

Bouatra, S., Aziat, F., Mandal, R., Guo, A.C., Wilson, M.R., Knox, C., Bjorndahl, T.C., Krishnamurthy, R., Saleem, F., Liu, P., Dame, Z.T., Poelzer, J., Huynh, J., Yallou, F.S., Psychogios, N., Dong, E., Bogumil, R., Roehring, C., Wishart, D.S., 2013. The Human Urine Metabolome. PLoS ONE 8, e73076. https://doi.org/10.1371/journal.pone.0073076

Dalla Marta, S., Novara, C., Giorgis, F., Bonifacio, A., Sergo, V., 2017. Optimization and Characterization of Paper-Made Surface Enhanced Raman Scattering (SERS) Substrates with Au and Ag NPs for Quantitative Analysis. Materials 10, 1365. https://doi.org/10.3390/ma10121365

Docter, D., Strieth, S., Westmeier, D., Hayden, O., Gao, M., Knauer, S.K., Stauber, R.H., 2015. No king without a crown - impact of the nanomaterial-protein corona on nanobiomedicine. Nanomedicine 10, 503-519. https://doi.org/10.2217/nnm.14.184

Dong, J., Chen, Q., Rong, C., Li, D., Rao, Y., 2011. Minimally Invasive Surface-Enhanced Raman Scattering Detection with Depth Profiles Based on a Surface-Enhanced Raman Scattering-Active Acupuncture Needle. Anal. Chem. 83, 6191-6195. https://doi.org/10.1021/ac2007009

Dong, J., Tao, Q., Guo, M., Yan, T., Qian, W., 2012. Glucose-responsive multifunctional acupuncture needle: A universal SERS detection strategy of small biomolecules in vivo. Anal. Methods 4, 3879-3883. https://doi.org/10.1039/C2AY25733E

F. Betz, J., W. Yu, W., Cheng, Y., M. White, I., W. Rubloff, G., 2014. Simple SERS substrates: powerful, portable, and full of potential. Physical Chemistry Chemical Physics 16, 2224-2239. https://doi.org/10.1039/C3CP53560F

Fleischmann, M., Hendra, P.J., McQuillan, A.J., 1974. Raman spectra of pyridine adsorbed at a silver electrode. Chemical Physics Letters 26, 163-166. https://doi.org/10.1016/0009-2614(74)85388-1

Fraire, J.C., Pérez, L.A., Coronado, E.A., 2013. Cluster Size Effects in the Surface-Enhanced Raman Scattering Response of Ag and Au Nanoparticle Aggregates: Experimental and Theoretical Insight. J. Phys. Chem. C 117, 23090-23107. https://doi.org/10.1021/jp3123709

Gao, X., Zheng, P., Kasani, S., Wu, S., Yang, F., Lewis, S., Nayeem, S., Engler-Chiurazzi, E.B., Wigginton, J.G., Simpkins, J.W., Wu, N., 2017. Paper-Based Surface-Enhanced Raman Scattering Lateral Flow Strip for Detection of Neuron-Specific Enolase in Blood Plasma. Anal. Chem. 89, 10104-10110. https://doi.org/10.1021/acs.analchem.7b03015

Gao, Y., Li, Y., Wang, Y., Chen, Y., Gu, J., Zhao, W., Ding, J., Shi, J., 2015. Controlled Synthesis of Multilayered Gold Nanoshells for Enhanced Photothermal Therapy and SERS Detection. Small 11, 77-83. https://doi.org/10.1002/smll.201402149

Garcia-Rico, E., Alvarez-Puebla, R.A., Guerrini, L., 2018. Direct surface-enhanced Raman scattering (SERS) spectroscopy of nucleic acids: from fundamental studies to real-life applications. Chem. Soc. Rev. 47, 49094923. https://doi.org/10.1039/C7CS00809K

Gebauer, J.S., Malissek, M., Simon, S., Knauer, S.K., Maskos, M., Stauber, R.H., Peukert, W., Treuel, L., 2012. Impact of the Nanoparticle-Protein Corona on Colloidal Stability and Protein Structure. Langmuir 28, 96739679. https://doi.org/10.1021/la301104a 
Genova, E., Pelin, M., Decorti, G., Stocco, G., Sergo, V., Ventura, A., Bonifacio, A., 2018. SERS of cells: What can we learn from cell lysates? Analytica Chimica Acta 1005, 93-100. https://doi.org/10.1016/j.aca.2017.12.002

Graham, D., Faulds, K., 2008. Quantitative SERRS for DNA sequence analysis. Chem. Soc. Rev. 37, 1042-1051. https://doi.org/10.1039/B707941A

Graham, D., Goodacre, R., Arnolds, H., Masson, J.-F., Schatz, G., Baumberg, J., Kim, D.-H., Aizpurua, J., Lum, W., Silvestri, A., Nijs, B. de, Xu, Y., Martino, G.D., Natan, M., Schlücker, S., Wuytens, P., Bruzas, I., Kuttner, C., Hardy, M., Chikkaraddy, R., Sabanés, N.M., Delfino, I., Dawson, P., Gawinkowski, S., Bontempi, N., Mahajan, S., Reich, S., Hourahine, B., Bell, S., Królikowska, A., Porter, M., Keeler, A., Kamp, M., Fountain, A., Fasolato, C., Giorgis, F., Otero, J.C., Matricardi, C., Duyne, R.V., Lombardi, J., Deckert, V., Velleman, L., 2017. Theory of SERS enhancement: general discussion. Faraday Discuss. 205, 173-211. https://doi.org/10.1039/C7FD90095C

Guerrero-Martínez, A., Barbosa, S., Pastoriza-Santos, I., Liz-Marzán, L.M., 2011. Nanostars shine bright for you: Colloidal synthesis, properties and applications of branched metallic nanoparticles. Current Opinion in Colloid \& Interface Science 16, 118-127. https://doi.org/10.1016/j.cocis.2010.12.007

Haiss, W., Thanh, N.T.K., Aveyard, J., Fernig, D.G., 2007. Determination of Size and Concentration of Gold Nanoparticles from UV-Vis Spectra. Anal. Chem. 79, 4215-4221. https://doi.org/10.1021/ac0702084

Hassoun, M., W.Schie, I., Tolstik, T., Stanca, S.E., Krafft, C., Popp, J., 2017. Surface-enhanced Raman spectroscopy of cell lysates mixed with silver nanoparticles for tumor classification. Beilstein Journal of Nanotechnology 8, 1183-1190. https://doi.org/10.3762/bjnano.8.120

Ho, Y.T., Azman, N. 'Ain, Loh, F.W.Y., Ong, G.K.T., Engudar, G., Kriz, S.A., Kah, J.C.Y., 2018. Protein Corona Formed from Different Blood Plasma Proteins Affects the Colloidal Stability of Nanoparticles Differently. Bioconjugate Chem. 29, 3923-3934. https://doi.org/10.1021/acs.bioconjchem.8b00743

Hole, P., Sillence, K., Hannell, C., Maguire, C.M., Roesslein, M., Suarez, G., Capracotta, S., Magdolenova, Z., Horev-Azaria, L., Dybowska, A., Cooke, L., Haase, A., Contal, S., Manø, S., Vennemann, A., Sauvain, J.-J., Staunton, K.C., Anguissola, S., Luch, A., Dusinska, M., Korenstein, R., Gutleb, A.C., Wiemann, M., Prina-Mello, A., Riediker, M., Wick, P., 2013. Interlaboratory comparison of size measurements on nanoparticles using nanoparticle tracking analysis (NTA). J Nanopart Res 15, 2101. https://doi.org/10.1007/s11051-013-2101-8

Hong, S., Li, X., 2013. Optimal Size of Gold Nanoparticles for Surface-Enhanced Raman Spectroscopy under Different Conditions [WWW Document]. Journal of Nanomaterials. https://doi.org/10.1155/2013/790323

Hoppmann, E.P., Yu, W.W., White, I.M., 2013. Highly sensitive and flexible inkjet printed SERS sensors on paper. Methods, Biosensor Technologies 63, 219-224. https://doi.org/10.1016/j.ymeth.2013.07.010

Hulteen, J.C., Van Duyne, R.P., 1995. Nanosphere lithography: A materials general fabrication process for periodic particle array surfaces. Journal of Vacuum Science \& Technology A 13, 1553-1558. https://doi.org/10.1116/1.579726

Jeanmaire, D.L., Van Duyne, R.P., 1977. Surface raman spectroelectrochemistry: Part I. Heterocyclic, aromatic, and aliphatic amines adsorbed on the anodized silver electrode. Journal of Electroanalytical Chemistry and Interfacial Electrochemistry 84, 1-20. https://doi.org/10.1016/S0022-0728(77)80224-6

Jiang, C., Wang, Y., Song, W., Lu, L., 2019. Delineating the tumor margin with intraoperative surface-enhanced Raman spectroscopy. Anal Bioanal Chem. https://doi.org/10.1007/s00216-019-01577-9 
J. Orendorff, C., Gearheart, L., R. Jana, N., J. Murphy, C., 2006. Aspect ratio dependence on surface enhanced Raman scattering using silver and gold nanorod substrates. Physical Chemistry Chemical Physics 8, 165-170. https://doi.org/10.1039/B512573A

Khoury, C.G., Vo-Dinh, T., 2008. Gold Nanostars For Surface-Enhanced Raman Scattering: Synthesis, Characterization and Optimization. J. Phys. Chem. C 112, 18849-18859. https://doi.org/10.1021/jp8054747

Kimling, J., Maier, M., Okenve, B., Kotaidis, V., Ballot, H., Plech, A., 2006. Turkevich Method for Gold Nanoparticle Synthesis Revisited. J. Phys. Chem. B 110, 15700-15707. https://doi.org/10.1021/jp061667w

Kneipp, J., Drescher, D., 2014. SERS in Cells: from Concepts to Practical Applications, in: Frontiers of SurfaceEnhanced Raman Scattering. John Wiley \& Sons, Ltd, pp. 285-308. https://doi.org/10.1002/9781118703601.ch13

Kneipp, J., Kneipp, H., Kneipp, K., McLaughlin, M., Brown, D., 2007. Surface-Enhanced Raman Scattering for Investigations of Eukaryotic Cells, in: Biomedical Vibrational Spectroscopy. John Wiley \& Sons, Ltd, pp. 243261. https://doi.org/10.1002/9780470283172.ch11

Koh, A.L., Bao, K., Khan, I., Smith, W.E., Kothleitner, G., Nordlander, P., Maier, S.A., McComb, D.W., 2009. Electron Energy-Loss Spectroscopy (EELS) of Surface Plasmons in Single Silver Nanoparticles and Dimers: Influence of Beam Damage and Mapping of Dark Modes. ACS Nano 3, 3015-3022. https://doi.org/10.1021/nn900922z

Kolluru, C., Gupta, R., Jiang, Q., Williams, M., Gholami Derami, H., Cao, S., Noel, R.K., Singamaneni, S., Prausnitz, M.R., 2019. Plasmonic Paper Microneedle Patch for On-Patch Detection of Molecules in Dermal Interstitial Fluid. ACS Sens. https://doi.org/10.1021/acssensors.9b00258

Kostrewa, S., Emgenbroich, M., Klockow, D., Wulff, G., 2003. Surface-Enhanced Raman Scattering on Molecularly Imprinted Polymers in Water. Macromolecular Chemistry and Physics 204, 481-487. https://doi.org/10.1002/macp.200390015

Laing, S., Jamieson, L.E., Faulds, K., Graham, D., 2017. Surface-enhanced Raman spectroscopy for in vivo biosensing. Nature Reviews Chemistry 1, 0060. https://doi.org/10.1038/s41570-017-0060

Lane, L.A., Xue, R., Nie, S., 2018. Emergence of two near-infrared windows for in vivo and intraoperative SERS. Current Opinion in Chemical Biology, Molecular Imaging / Chemical Genetics and Epigenetics 45, 95-103. https://doi.org/10.1016/j.cbpa.2018.03.015

Larkin, P., 2011. Infrared and Raman Spectroscopy; Principles and Spectral Interpretation. Elsevier.

Le Ru, E.C., Blackie, E., Meyer, M., Etchegoin, P.G., 2007. Surface Enhanced Raman Scattering Enhancement Factors: A Comprehensive Study. J. Phys. Chem. C 111, 13794-13803. https://doi.org/10.1021/jp0687908

Lee, I., Han, S.W., Kim, K., 2001. Simultaneous preparation of SERS-active metal colloids and plates by laser ablation. Journal of Raman Spectroscopy 32, 947-952. https://doi.org/10.1002/jrs.781

Lee, P.C., Meisel, D., 1982. Adsorption and surface-enhanced Raman of dyes on silver and gold sols. J. Phys. Chem. 86, 3391-3395. https://doi.org/10.1021/j100214a025

Leopold, N., Lendl, B., 2003. A New Method for Fast Preparation of Highly Surface-Enhanced Raman Scattering (SERS) Active Silver Colloids at Room Temperature by Reduction of Silver Nitrate with Hydroxylamine Hydrochloride. The Journal of Physical Chemistry B 107, 5723-5727. https://doi.org/10.1021/jp027460u

Li, P., Zhou, B., Cao, X., Tang, X., Yang, L., Hu, L., Liu, J., 2017. Functionalized Acupuncture Needle as SurfaceEnhanced Resonance Raman Spectroscopy Sensor for Rapid and Sensitive Detection of Dopamine in Serum 
and Cerebrospinal Fluid. Chemistry - A European Journal 23, 14278-14285. https://doi.org/10.1002/chem.201702607

Li, W., Zhao, X., Yi, Z., Glushenkov, A.M., Kong, L., 2017. Plasmonic substrates for surface enhanced Raman scattering. Analytica Chimica Acta 984, 19-41. https://doi.org/10.1016/j.aca.2017.06.002

L. Kleinman, S., R. Frontiera, R., Henry, A.-I., A. Dieringer, J., Duyne, R.P.V., 2013. Creating, characterizing, and controlling chemistry with SERS hot spots. Physical Chemistry Chemical Physics 15, 21-36. https://doi.org/10.1039/C2CP42598J

Long, D.A., 2002. The Raman Effect: A Unified Treatment of the Theory of Raman Scattering by Molecules. Wiley.

Lu, W., Singh, A.K., Khan, S.A., Senapati, D., Yu, H., Ray, P.C., 2010. Gold Nano-Popcorn-Based Targeted Diagnosis, Nanotherapy Treatment, and In Situ Monitoring of Photothermal Therapy Response of Prostate Cancer Cells Using Surface-Enhanced Raman Spectroscopy. J. Am. Chem. Soc. 132, 18103-18114. https://doi.org/10.1021/ja104924b

Lussier, F., Brulé, T., Vishwakarma, M., Das, T., Spatz, J.P., Masson, J.-F., 2016. Dynamic-SERS Optophysiology: A Nanosensor for Monitoring Cell Secretion Events. Nano Lett. 16, 3866-3871. https://doi.org/10.1021/acs.nanolett.6b01371

Lyandres, O., Yuen, J.M., Shah, N.C., VanDuyne, R.P., Walsh, J.T., Glucksberg, M.R., 2008. Progress Toward an In Vivo Surface-Enhanced Raman Spectroscopy Glucose Sensor. Diabetes Technology \& Therapeutics 10, 257-265. https://doi.org/10.1089/dia.2007.0288

Ma, K., Yuen, J.M., Shah, N.C., Walsh, J.T., Glucksberg, M.R., Van Duyne, R.P., 2011. In Vivo, Transcutaneous Glucose Sensing Using Surface-Enhanced Spatially Offset Raman Spectroscopy: Multiple Rats, Improved Hypoglycemic Accuracy, Low Incident Power, and Continuous Monitoring for Greater than 17 Days. Anal. Chem. 83, 9146-9152. https://doi.org/10.1021/ac202343e

Maltzahn, G. von, Centrone, A., Park, J.-H., Ramanathan, R., Sailor, M.J., Hatton, T.A., Bhatia, S.N., 2009. SERSCoded Gold Nanorods as a Multifunctional Platform for Densely Multiplexed Near-Infrared Imaging and Photothermal Heating. Advanced Materials 21, 3175-3180. https://doi.org/10.1002/adma.200803464

Marks, H., Schechinger, M., Garza, J., Locke, A., Coté, G., 2017. Surface enhanced Raman spectroscopy (SERS) for in vitro diagnostic testing at the point of care. Nanophotonics 6, 681-701. https://doi.org/10.1515/nanoph-2016-0180

Marsich, L., Bonifacio, A., Mandal, S., Krol, S., Beleites, C., Sergo, V., 2012. Poly-L-lysine-Coated Silver Nanoparticles as Positively Charged Substrates for Surface-Enhanced Raman Scattering. Langmuir 28, 1316613171. https://doi.org/10.1021/la302383r

McNay, G., Eustace, D., Smith, W.E., Faulds, K., Graham, D., 2011. Surface-enhanced Raman scattering (SERS) and surface-enhanced resonance raman scattering (SERRS): A review of applications. Applied Spectroscopy 65, 825-837. https://doi.org/10.1366/11-06365

Mosier-Boss, P.A., 2017. Review of SERS Substrates for Chemical Sensing. Nanomaterials 7, 142. https://doi.org/10.3390/nano7060142

Negri, P., Marotta, N.E., Bottomley, L.A., Dluhy, R.A., 2011. Removal of Surface Contamination and SelfAssembled Monolayers (SAMs) from Silver (Ag) Nanorod Substrates by Plasma Cleaning with Argon. Appl. Spectrosc., AS 65, 66-74. 
Nel, A.E., Mädler, L., Velegol, D., Xia, T., Hoek, E.M.V., Somasundaran, P., Klaessig, F., Castranova, V., Thompson, M., 2009. Understanding biophysicochemical interactions at the nano-bio interface. Nature Materials 8, 543-557. https://doi.org/10.1038/nmat2442

Njoki, P.N., Lim, I.-I.S., Mott, D., Park, H.-Y., Khan, B., Mishra, S., Sujakumar, R., Luo, J., Zhong, C.-J., 2007. Size Correlation of Optical and Spectroscopic Properties for Gold Nanoparticles. J. Phys. Chem. C 111, 1466414669. https://doi.org/10.1021/jp074902z

O'Connor, D.J., Sexton, B.A., Smart, R.S.C., 2013. Surface Analysis Methods in Materials Science. Springer Science \& Business Media.

Petryayeva, E., Krull, U.J., 2011. Localized surface plasmon resonance: Nanostructures, bioassays and biosensing-A review. Analytica Chimica Acta 706, 8-24. https://doi.org/10.1016/j.aca.2011.08.020

Piella, J., Bastús, N.G., Puntes, V., 2017. Size-Dependent Protein-Nanoparticle Interactions in CitrateStabilized Gold Nanoparticles: The Emergence of the Protein Corona. Bioconjugate Chem. 28, 88-97. https://doi.org/10.1021/acs.bioconjchem.6b00575

Prochazka, M., 2015. Surface-Enhanced Raman Spectroscopy: Bioanalytical, Biomolecular and Medical Applications. Springer.

Psychogios, N., Hau, D.D., Peng, J., Guo, A.C., Mandal, R., Bouatra, S., Sinelnikov, I., Krishnamurthy, R., Eisner, R., Gautam, B., Young, N., Xia, J., Knox, C., Dong, E., Huang, P., Hollander, Z., Pedersen, T.L., Smith, S.R., Bamforth, F., Greiner, R., McManus, B., Newman, J.W., Goodfriend, T., Wishart, D.S., 2011. The Human Serum Metabolome. PLoS ONE 6, e16957. https://doi.org/10.1371/journal.pone.0016957

Raman, C.V., Krishnan, K.S., 1928. A New Type of Secondary Radiation. Nature 121, 501. https://doi.org/10.1038/121501c0

Restaino, S.M., White, I.M., 2019. A critical review of flexible and porous SERS sensors for analytical chemistry at the point-of-sample. Analytica Chimica Acta 1060, 17-29. https://doi.org/10.1016/j.aca.2018.11.057

Roth, E., Hope, G.A., Schweinsberg, D.P., Kiefer, W., Fredericks, P.M., 1993. Simple Technique for Measuring Surface-Enhanced Fourier Transform Raman Spectra of Organic Compounds. Appl. Spectrosc., AS 47, 17941800.

Ru, E.L., Etchegoin, P., 2008. Principles of Surface-Enhanced Raman Spectroscopy: and related plasmonic effects. Elsevier.

Rycenga, M., Wang, Z., Gordon, E., Cobley, C.M., Schwartz, A.G., Lo, C.S., Xia, Y., 2009. Probing the Photothermal Effect of Gold-Based Nanocages with Surface-Enhanced Raman Scattering (SERS). Angewandte Chemie International Edition 48, 9924-9927. https://doi.org/10.1002/anie.200904382

Sabatté, G., Keir, R., Lawlor, M., Black, M., Graham, D., Smith, W.E., 2008. Comparison of Surface-Enhanced Resonance Raman Scattering and Fluorescence for Detection of a Labeled Antibody. Anal. Chem. 80, 23512356. https://doi.org/10.1021/ac071343j

Sadate, S., Calzzani, F.A., Kassu, A., Sharma, A., Ruffin, P.B., Brantley, C., Edwards, E., 2010. Recycling of surface-enhanced Raman substrates by ultraviolet cleaning. OE 49, 106501. https://doi.org/10.1117/1.3491201

Sánchez-Cortés, S., García-Ramos, J.V., 1998. Anomalous Raman bands appearing in surface-enhanced Raman spectra. Journal of Raman Spectroscopy 29, 365-371. https://doi.org/10.1002/(SICI)10974555(199805)29:5<365::AID-JRS247>3.0.CO;2-Y 
Schlücker, S., 2011. Surface Enhanced Raman Spectroscopy: Analytical, Biophysical and Life Science Applications. John Wiley \& Sons.

Schmidt, M.S., Hübner, J., Boisen, A., 2012. Large Area Fabrication of Leaning Silicon Nanopillars for Surface Enhanced Raman Spectroscopy. Advanced Materials 24, OP11-OP18. https://doi.org/10.1002/adma.201103496

Sharma, B., Bugga, P., Madison, L.R., Henry, A.-I., Blaber, M.G., Greeneltch, N.G., Chiang, N., Mrksich, M., Schatz, G.C., Van Duyne, R.P., 2016. Bisboronic Acids for Selective, Physiologically Relevant Direct Glucose Sensing with Surface-Enhanced Raman Spectroscopy. J. Am. Chem. Soc. 138, 13952-13959. https://doi.org/10.1021/jacs.6b07331

Siegfried, T., Kind, M., Terfort, A., Martin, O.J.F., Zharnikov, M., Ballav, N., Sigg, H., 2013. Reusable plasmonic substrates fabricated by interference lithography: a platform for systematic sensing studies. Journal of Raman Spectroscopy 44, 170-175. https://doi.org/10.1002/jrs.4163

Smekal, A., 1923. Zur Quantentheorie der Dispersion. Naturwissenschaften 11, 873-875. https://doi.org/10.1007/BF01576902

Smith, E., Dent, G., 2013. Modern Raman Spectroscopy: A Practical Approach. John Wiley \& Sons.

Stamplecoskie, K.G., Scaiano, J.C., Tiwari, V.S., Anis, H., 2011. Optimal Size of Silver Nanoparticles for SurfaceEnhanced Raman Spectroscopy. J. Phys. Chem. C 115, 1403-1409. https://doi.org/10.1021/jp106666t

Stone, N., Kerssens, M., Rhys Lloyd, G., Faulds, K., Graham, D., Matousek, P., 2011. Surface enhanced spatially offset Raman spectroscopic (SESORS) imaging - the next dimension. Chemical Science 2, 776-780. https://doi.org/10.1039/C0SC00570C

Stuart, D.A., Yuen, J.M., Shah, N., Lyandres, O., Yonzon, C.R., Glucksberg, M.R., Walsh, J.T., Van Duyne, R.P., 2006. In Vivo Glucose Measurement by Surface-Enhanced Raman Spectroscopy. Anal. Chem. 78, 7211-7215. https://doi.org/10.1021/ac061238u

Sun, F., Hung, H.-C., Sinclair, A., Zhang, P., Bai, T., Galvan, D.D., Jain, P., Li, B., Jiang, S., Yu, Q., 2016. Hierarchical zwitterionic modification of a SERS substrate enables real-time drug monitoring in blood plasma. Nature Communications 7, 13437. https://doi.org/10.1038/ncomms13437

Sun, X., Stagon, S., Huang, H., Chen, J., Lei, Y., 2014. Functionalized aligned silver nanorod arrays for glucose sensing through surface enhanced Raman scattering. RSC Adv. 4, 23382-23388. https://doi.org/10.1039/C4RA02423K

Taylor, J., Huefner, A., Li, L., Wingfield, J., Mahajan, S., 2016. Nanoparticles and intracellular applications of surface-enhanced Raman spectroscopy. The Analyst 141, 5037-5055. https://doi.org/10.1039/C6AN01003B

Tran, V., Walkenfort, B., König, M., Salehi, M., Schlücker, S., 2019. Rapid, Quantitative, and Ultrasensitive Point-of-Care Testing: A Portable SERS Reader for Lateral Flow Assays in Clinical Chemistry. Angewandte Chemie International Edition 58, 442-446. https://doi.org/10.1002/anie.201810917

Vandenabeele, P., 2013. Practical Raman Spectroscopy: An Introduction. John Wiley \& Sons.

Virga, A., Rivolo, P., Frascella, F., Angelini, A., Descrovi, E., Geobaldo, F., Giorgis, F., 2013. Silver Nanoparticles on Porous Silicon: Approaching Single Molecule Detection in Resonant SERS Regime. J. Phys. Chem. C 117, 20139-20145. https://doi.org/10.1021/jp405117p

Vo-Dinh, T., Fales, A.M., Griffin, G.D., Khoury, C.G., Liu, Y., Ngo, H., Norton, S.J., Register, J.K., Wang, H.-N., Yuan, H., 2013. Plasmonic nanoprobes: from chemical sensing to medical diagnostics and therapy. Nanoscale 5, 10127-10140. https://doi.org/10.1039/C3NR03633B 
Wang, X., Qian, X., Beitler, J.J., Chen, Z.G., Khuri, F.R., Lewis, M.M., Shin, H.J.C., Nie, S., Shin, D.M., 2011. Detection of Circulating Tumor Cells in Human Peripheral Blood Using Surface-Enhanced Raman Scattering Nanoparticles. Cancer Res 71, 1526-1532. https://doi.org/10.1158/0008-5472.CAN-10-3069

Wang, Y.W., Kang, S., Khan, A., Bao, P.Q., Liu, J.T.C., 2015. In vivo multiplexed molecular imaging of esophageal cancer via spectral endoscopy of topically applied SERS nanoparticles. Biomed. Opt. Express, BOE 6, 3714-3723. https://doi.org/10.1364/BOE.6.003714

Wang, Y.W., Khan, A., Leigh, S.Y., Wang, D., Chen, Y., Meza, D., Liu, J.T.C., 2014. Comprehensive spectral endoscopy of topically applied SERS nanoparticles in the rat esophagus. Biomed. Opt. Express, BOE 5, 28832895. https://doi.org/10.1364/BOE.5.002883

Wei, X., Su, S., Guo, Y., Jiang, X., Zhong, Y., Su, Y., Fan, C., Lee, S.-T., He, Y., 2013. A Molecular Beacon-Based Signal-Off Surface-Enhanced Raman Scattering Strategy for Highly Sensitive, Reproducible, and Multiplexed DNA Detection. Small 9, 2493-2499. https://doi.org/10.1002/smll.201202914

Weng, G., Yang, Y., Zhao, Jing, Zhu, J., Li, J., Zhao, Junwu, 2018. Preparation and SERS performance of Au NP/paper strips based on inkjet printing and seed mediated growth: The effect of silver ions. Solid State Communications 272, 67-73. https://doi.org/10.1016/j.ssc.2018.01.014

Wu, X., Luo, L., Yang, S., Ma, X., Li, Y., Dong, C., Tian, Y., Zhang, L., Shen, Z., Wu, A., 2015. Improved SERS Nanoparticles for Direct Detection of Circulating Tumor Cells in the Blood. ACS Appl. Mater. Interfaces 7 , 9965-9971. https://doi.org/10.1021/acsami.5b02276

Yonzon, C.R., Lyandres, O., Shah, N.C., Dieringer, J.A., Van Duyne, R.P., 2006. Glucose Sensing with SurfaceEnhanced Raman Spectroscopy, in: Kneipp, K., Moskovits, M., Kneipp, H. (Eds.), Surface-Enhanced Raman Scattering: Physics and Applications, Topics in Applied Physics. Springer Berlin Heidelberg, Berlin, Heidelberg, pp. 367-379. https://doi.org/10.1007/3-540-33567-6_19

Yuen, C., Liu, Q., 2014. Towards in vivo intradermal surface enhanced Raman scattering (SERS) measurements: silver coated microneedle based SERS probe. Journal of Biophotonics 7, 683-689. https://doi.org/10.1002/jbio.201300006

Zavaleta, C.L., Garai, E., Liu, J.T.C., Sensarn, S., Mandella, M.J., Sompel, D.V. de, Friedland, S., Dam, J.V., Contag, C.H., Gambhir, S.S., 2013. A Raman-based endoscopic strategy for multiplexed molecular imaging. PNAS 110, E2288-E2297. https://doi.org/10.1073/pnas.1211309110

Zhang, Y., Walkenfort, B., Yoon, J.H., Schlücker, S., Xie, W., 2015. Gold and silver nanoparticle monomers are non-SERS-active: a negative experimental study with silica-encapsulated Raman-reporter-coated metal colloids. Phys. Chem. Chem. Phys. https://doi.org/10.1039/C4CP05073H 


\section{ELECTRO- AND NON- ELECTRO ASSISTED SPINNING TECHNOLOGIES FOR IN VITRO AND IN VIVO MODELS}

Janeth Serrano Bello $^{1++}$, Iriczalli Cruz-Maya ${ }^{2,3++}$, Patricia González-Alva ${ }^{1}$, Marco A. Alvarez-Perez ${ }^{1 *}$ and Vincenzo Guarino ${ }^{2 *}$

1 Tissue Bioengineering Laboratory, DEPeI, School of Dentistry, Universidad Nacional Autonoma de Mexico (UNAM), Circuito Exterior s/n C.P. 04510 Coyoacán, Mexico DF, Mexico 2 IPCB/CNR, Institute of Polymers, Composites and Biomaterials - Consiglio Nazionale delle Ricerche, Mostra D’Oltremare, Pad. 20, V.le J.F. Kennedy 54, 80125, Naples, Italy. 3 Department of Chemical, Materials and Industrial Production Engineering, University of Naples Federico II, Naples, Italy

++ equally contributed authors

Corresponding authors:

Marco Alvarez: marcoalv@unam.mx

Vincenzo Guarino: vguarino@unina.it:; vincenzo.guarino@cnr.it 


\section{List of contents:}

Abstract

1. Introduction:

2. Electro assisted technologies

\subsection{Electrospinning}

3. Not electro assisted technologies

\subsection{Air Jet spinning}

4. Optimization of nanofiber fabrication

4.1 Definition of process conditions

4.2 Selection of solution properties

4.3 Environmental conditions

5. In vitro applications

5.1 Tissue engineering

5.2 Molecular delivery

6. In vivo models 


\section{Introduction}

The use of fibers has gained attention in biomedical applications, due to their ability to mimic the extracellular matrix of native tissues and more accurately controlling the release of biomolecules via in order to enhance the regeneration process of damage tissues [1]. In tissue engineering, fibres can be successfully assembled to form micor/nanostructured scaffolds with peculiar topographic cues and composition suitable to support cells to growth and proliferation in vitro. In the last years, different strategies have been developed to fabricate micro- and sub-micrometric fibers for large scale productions, including phase-separation, self-assembly, melt spinning, electrospinning and air-jet spinning. In this chapter, the electrospinning technique will be described as the most widely used technique to produce fibers because allows fabricating fibers at different size scale by setting a large combination of process parameters, solution properties and environmental factors. Moreover, air-jet spinning or solution blow spinning will be introduced as an emerging technique that allows manipulating polymer solutions by the use of air or gas pressure to produce fibers [2].

\section{Electro-assisted spinning}

3.

\subsection{Pure electrospinning}

The basic principles of the electrospinning technique is based on the stretching of viscoelastic solution to form fibers by the application of electrostatic forces generated via high voltage electric fields. Final morphological properties of the fibres can be optimized by controlling several factors that can affect the final size, morphology and properties of fibers. These factors can be classified in three main groups: process parameters, solution properties and environmental conditions for both techniques (Table 1).

The electrospinning setup is mainly comprised of a syringe with a metallic tip to contain the polymer solution, a power supply and the grounded collector [14]. The electrospinning (ES) process begins 
the polymer solution is delivered in a constant flow rate by the syringe pump. When polymer solution interacts with a high voltage through the metallic needle, a polymer jet ejects from needle to the collector. The interaction between polymer solution and high applied voltage overcomes the surface tension forces of solution through repulsive electrical forces generated when the electrical field reaches to a critical value. The increase on the electric field, deforms the droplet to a conical shape, called Taylor cone, in the tip of the needle. Once the Taylor cone is formed, a stable jet is ejected in direction to the collector, kept at an adjusted distance. The travel between the tip and collector leads to evaporation of solvent, leaving the polymer fibers [15].

The increased use of electrospinning technology has addressed to develop several variations as orientation of fibers, co-electrospinning or co-axial electrospinning, to enhance the productivity, add functionality and improve the quality of fibers, and combining the knowledge on electrospinning to form particles in a process named electrospray $[3,16,17]$.

\subsection{Electro fluid dynamic tecnologies}

Electrospinning takes part of the group of electrofluidodynamic techniques (EFDTs), that are highly flexible and low-cost processes suitable to manipulate biomaterials via electrostatic forces. Conventionally, electrospun fibers can be successfully used to design porous scaffolds with a fully interconnected structure able to facilitate cell migration, high surface area for oxygen permeability and abroad of spatial arrangements to mimic the native tissues and enhance the regeneration process. Recent progresses in the nanotechnologies are currently addressing towards the design of multicomponent biomaterials - e.g., combining fibers and/or particles obtained by the basic principles of electrospinning/electrospraying, thus offering the opportunity to include selected molecular signals able to improve the bioactive response of the scaffold $[3,4]$.

Among them, coaxial electrospinning currently represents an interesting strategy to develop coreshell fibers and/or particles with a controlled release of hydrophilic/hydrophobic molecules and higher protection of labile molecules [5]. To combine the advantages of fibers with particles for tissue engineering and drug release, combined processes based on electrospraying and/or electrospinning 
techniques have been explored. [6,7]. In these cases, two different solutions can be pumped simultaneously or sequentially to form a multicomponent platform composed of net fibers and loaded fibre and/or particles to control their release and providing morphological/structural signals to cells, suitable for tissue engineering.

Recently additive manufacturing technologies (AMT) have been developed to fabricate ordered fibrous scaffolds [8]. For instance, melt-electrospinning and cryogenic electrospinning include additive technologies which use high or low temperatures to influence polymer solution properties in order to obtain different fiber patterned structures. In the case of melt-electrospinning, the interaction of melt polymers with the electrostatic forces allows generation of 3D scaffolds without using aggressive organic solvents [9,10]. Meanwhile cryogenic electrospinning or low-temperature electrospinning allows nucleating ice crystals into the forming fibres that, after their removal, result in the formation of highly controlled porosity into fibres [11].

Lastly, there are strategies for the modification of fibers surface once obtained by electrospinning. Between the post-treatments of electrospun fibers are laser-based processes and nanoimprinting lithography to create specific patterns on fibers, which can influence on morphology cell and migration. To obtain a more porous scaffold, thermal induced self-agglomeration (TISA) has been used to form a 3D structure, with high porosity and interconnected macropores for tissue engineering applications $[12,13]$.

\section{Non electro-assisted spinning}

Non-electro-assisted spinning include a group of low-cost technique that allow to assembly polymeric fibers by the application of shear and pressure gradients at the interface gas-solution. This process involves the stretching of the polymer solution by applying a gas flow (air, nitrogen, argon, etc) at high pressure (30 to $60 \mathrm{psi}$ ) regulated in a constant speed, able to stretch the polymer into fibers at the needle tip. The process mainly depends upon the solvent evaporation rate that allow reaching the 
solidification of the polymer fibre, at a controlled diameter on the micro or sub-micrometric size scale In order to optimize the fibre morphology, the control of some solution parameters - such as the viscosity, concentration and solvent permittivity - and processing parameters including and the gap - it means the distance between the needle and the collector - or nozzle size, is crucial, similarly to the case of electrospinning. However, the main advantage of non-electro assisted technologies is that they can work with higher rate of production, allowing to coat large areas in a faster time and on a great variety of substrates, as metals, ceramic or non-conductive scaffolds [21-23]. In the field of tissue engineering, non-electro assisted spinning techniques are a serious alternative technology to fabricate scaffolds with micro- and nano- fibers and random or aligned spatial distribution, able to mimic extracellular matrix (ECM) features [24-26]. Besides, fibre morphology plays an important role in tissue engineering because the cells could sense and mediating the cell adhesion, proliferation and differentiation improving the biological respond of the cells. Several reports synthesized fibre or composites spun mats by the non-electrospinning mode and evaluated the biological response of mesenchymal stem cells (MSC). The polymeric fibre and composite mats showed good cell-material interaction that allow to cell spreading and penetrated into the surface, supporting the cell adhesion, cell proliferation and the most important improving the differentiation to osteogenic phenotype [2732]. More recently, the non-electrospinning mode area used to coat of $3 \mathrm{D}$ tubular surface scaffold because the fibers could be sprayed rapidly covering in less than $1 \mathrm{~min}$ the entire surface of the 3D printed scaffold showing an enhanced cell respond of osteoblasts cells with application on bone tissue engineering [33]. Lastly, non-electro assisted spinning techniques can offer the opportunity to variously combine fibre spun mats with nanoparticles, biomolecules, drugs or cells thus resulting a high versatile technique to design bioinspired platforms for tissue engineering [34-37].

\subsection{Air jet spinning}

A large variety of techniques has been studied to design biomaterials for both, tissue engineering and drug delivery systems. Among them, Air-jet spinning (AJS) known also as solution blow spinning 
(SBS). The equipment required for AJS consists on a gas source, such as nitrogen, argon or air, a pressure regulator, a specialized nozzle system, whether a commercially available airbrush or custom airbrush and the collector. The AJS systems is based on the use of concentric nozzle system, in which polymer solution is pumped at a constant rate into the inner nozzle, and high-pressure gas is delivered in the outer nozzle simultaneously to stretch the droplet of polymer solution to form fibers [38]. When the droplet is at the tip of inner nozzle, the air delivered from the outer nozzle helps to deform the polymer solution into a conical shape, similar to Taylor cone in electrospinning process. When the critical pressure exceeds, the jet ejects from the cone to the collector. As in electrospinning process, the flight to the collector, allows the evaporation of solvents creating solid fibers [39].

As in case of electrospinning, different approaches have been explored in terms of the equipment, considering two different designs for AJS, commercially airbrush and custom-built airbrush. The custom airbrush uses one characteristic element of electrospinning setup, the syringe pump, which may allow more comparable the quality of fibers between both techniques [40,41].

\section{Optimization of nanofiber fabrication}

\subsection{Definition of process conditions}

Considering that stretching of polymer solution is induced differently in ES and AJS, should be consider two main different process parameters, applied voltage and air pressure, respectively. There are process parameters, as tip-to-collector distance, flow rate, needle diameter, that influence equally ES and AJS technologies.

a) Voltage (for electrospinning):

Electrospinning process is a unique approach based on the use of electrostatic forces to produce fibers from polymer solutions. The strength of the solution by applied electric field leads to form fibers, defects in the fibers or lack of the process. Several studies had explored the shape and defects formed using higher or lower voltages, which is related with the jet stability. The instability causes a change 
of shape in the initial jet, thus, affecting the morphology of fibers, where can result on presence or not of beaded fibers when is applied lower or higher voltage, respectively [42]. Several studies have observed the effect of voltage on the fibers' diameter, concluding that increasing the voltage, allows the increase of fibers diameter, with relatively uniform distribution when other parameters are constant $[43,44]$. The effect of voltage in diameter of fibers, has been explained by the interaction of three major forces, it means the Coulombic, the viscoelastic and the surface tension. Beads may appear when voltage is low, because Coulombic force is not enough respect to surface tension. An increase of voltage generates a greater Coulombic force than viscoelastic force, however may result in the over-stretched charged jet, and a faster travel of it to the collector, preventing the proper evaporation of solvent, creating fibers with large diameters but irregular morphology [45]. Regarding to fiber shape, there are studies suggesting that increasing the applied voltage, creates shape transition from round to flat/ribbon fibers [46]. To stablish the threshold value of applied voltage, it is necessary to considerer the solution properties, as solvent, polymer concentration and molecular weight to find the optimal parameter for the obtention of fibers.

\section{b) air pressure (for blow spinning)}

AJS, uses air or gas pressure flowing on the outer nozzle of the system. The use of air pressure helps to deform solution streams, evaporate solvent, and solidifying them into fibers. The morphology of fibers is influenced by the effect of air pressure which should be controlled by a manometer to maintain the pressure constant. It has been shown that air pressure has a linear influence on fibers diameters, the range reported is between 30 and 90 psi, depending of polymer solution properties. It has been shown that pressures under 30 psi are not able to overcome the surface tension to produce fibers, meanwhile higher pressures, fibers are ejected at high velocity from the nozzle, which does not allow the evaporation of solvent [47]. Due to high air pressure, there is a stronger shearing force between the gas and solution interface when all the other parameters were fixed. Conversely, when low gas pressure is applied, the solution is not stretched, and solvent evaporation is not allowed. 
However, it has to be pay attention on higher pressures because may be a waste of gas and polymer solution due to fibers start to fly out of the working zone.

\section{c) Distance}

Distance between needle tip and collector is an important parameter to encourage the optimum evaporation of solvent in AJS and ES technologies and control the morphology and diameter of fibers. Generally, it has been found that shorter distances result on wet fibers deposition, which instead generate fibers, film surface or beads are obtained, due to the dryness of the solvent used in the polymer solution. For instance, long distance may to be appropriate for evaporation of solvents, however, is in function of the boiling point of solvent used [48]. Particularly, in AJS higher distances have been used when solvents have higher boiling points as water or acetic acid, where used distance are about 50 or $60 \mathrm{~cm}$, differently in case of chloroform or acetone where shorter distances allowed correct evaporation $[29,49]$. In ES process, has been found that minimum distance is required for the correct evaporation of solvents, resulting in free defects fibers, with large diameters, and also increased the polydispersity of nanofibers diameters [50,51]. However, in some cases, distances too large or too short can form beads or beaded fibers, meanwhile in other cases distance does not affect the morphology $[52,53]$.

In otherwise, the effect of distance in ES may affect the field strength, if distance decreases, the field strength is higher, generating the same effect of an increased applied voltage $[41,54]$.

\section{d) Flow rate}

The rate at which the polymeric solution passes through the needle has effect on the morphology of fibers. This parameter depends of the polymeric system, however, in electrospinning are preferred slow rates to maintain a stable jet cone, contrary to AJS, even if at high flow rates the amount of electrospun material can be increased. In case of ES, the feed rate is a controllable parameter by a syringe pump that maintains the flow of solution during the time of deposition. Studies with different polymer solutions, shown that electric current increases, while volume charge density decreases as a function of flow rate [55]. In order to maintain the volume charge density and stable Taylor cone, 
the flow rate should be adjusted to obtain a narrowest fiber diameter distribution. [48,56] The diameter of fibers is increased with the flow rate, however if overcome a critical value, the fibers present defects as beads or ribbon-like structures due to the incompletely evaporation of solvent $[53,57]$.

For AJS, the control of flow rate may be different depending of the airbrush device. The feed rate in a commercial airbrush, is determined by measuring the known volume of polymer solution and the time deposition, which means, in this case, is not a controllable parameter [32]. In the other hand, custom devices include a syringe pump, thus the feed rate is controllable as in case of ES, but usually used in higher rates, for instance, rates below $20 \mu \mathrm{L} / \mathrm{min}$ had resulted in intermittent flow at the nozzle. Higher feed rates in AJS may cause nozzle obstruction, because the droplet tend to clog at the needle tip. In the other wise, lower feed rates than the critical value, jet instability is detected when the solution dragged to the collector faster than the feed rate [58]. Comparing commercial and custom airbrush, in the same conditions regarding to pressure and polymer concentration, studies concluded that both devices are able to produce micro and nanofibers, however, custom airbrush offers more control and more reproducible conditions, even at higher polymer concentrations [40,41]. AJS is considered as high fiber production rate, however, is important to select carefully the solvent, because many fibers come out at the same time at high velocity, reaching in a wet phase to the collector [2,59].

\subsection{Definition of Solution properties}

Properties of polymers as molecular weight, concentration and viscosity are correlated to each other and influence the spinnability of work solution, as well size and morphology of electrospun and airbrushed fibers. In the other hand, solvent properties also play an important role related with their capability to dissolve the polymers to use and their evaporation rate for the fabrication of fibers. In addition, the conductivity of solution is an important parameter exclusively of ES process for the jet formation. 
Molecular weight of polymers influences the viscosity of solutions, thus affecting the morphology of fibers. The viscosity of solution is proportional to polymer concentration and may influence the morphology and diameter of fibers obtained by ES and AJS.

\section{a) Polymer concentration, molecular weight and viscosity}

Molecular weight and polymer concentration have an effect in the rheological and electrical properties as viscosity, surface tension and conductivity for fiber formation in both, ES and AJS. In general, a polymer with high molecular weight has a high viscosity in solution compared to the same polymer with lower molecular weight. For example, low molecular weight prevents the fibers formation, usually results on droplets or beaded fibers [48]. Contrary, the polymers with high molecular weight can generate uniform and bead-less fibers [60].

In other wise, polymer concentration, highly related to solution viscosity, has been widely studied by its influence in fiber morphology. However, there is no a clear rule about the concentration since it depends on the polymer and solvent characteristics For instance, optimal concentrations can be lower but for other polymers should be higher to form fibers without beads [53]. In general is considered that the cohesion forces between polymer chains the ratio between polymer concentration and polymer concentration at which overlapping occurs between polymer chains, should be above 6 [61]. The use of lower concentration in ES may generate microparticles, due to the applied voltage which breaks the polymer chains into small beads or particles, in a process known as electrospraying [5]. In AJS the same behavior has been detected, when low concentrations are used, some fibers and many defects can be obtained, but by increasing the concentration fibers can be obtained [2,23]. Besides fiber morphology, polymer concentration also plays a determinative roll in diameter of fibers. It has been reported that increasing the polymer concentration for AJS and ES process, there is a proportional increase on fiber diameter $[62,63]$. Increasing the polymer concentration, results in an increase of polymer chain entanglement, thus the viscosity of solution is also increased. However, higher polymer concentration than a critical value, the solution is not able to pass through the needle or dry in the tip resulting in defective fibers or even blocking the fiber formation $[18,50]$. Another 
interesting aspect is that beads show a shape change according to the viscosity of solution. This effect has been studied by different groups where concluded that beads shape changes from droplet to stretched droplet when viscosity changes from low to higher, respectively $[64,65]$.

\section{b) Solvents}

For the selection of solvent, two main aspects should be considered, the type of polymer to use and the boiling point of solvent. The volatility of solvents in both technologies is important to raise the evaporation of solvent during fiber deposition into the path between needle tip to the collector. Solvents with low boiling point are highly volatile, are mostly avoided due to the high evaporation rate, which could dry and block the needle tip. However, solvents less volatile, does not allow the solvent evaporation, resulting on deposition beaded fibers or deformation of fibers to a film. The evaporation rate of solvents may be controlled by decrease or increase the distance between needle tip and collector to obtain smooth fibers and controlling environmental factors as temperature and humidity. Another approach is the use of solvent systems, it means the use of two solvents with different boiling points, to improve the morphology of fibers [66]. In this context, the improvement of morphology fibers could be attributable to the addition of one solvent with higher boiling point, for example ethanol/water, acetone/DMAc or chloroform/MeOH solvent systems [64,67]. Furthermore, a solvent system could be used to prepare porous fibers by the phase separation of the non-solvent component when the polymer jet is on the way to the collector [53].

Among solution properties, conductivity is a parameter to consider in ES process to influence the stretching of polymer jet for production of fibers. A good conductivity of solution lead to form the Taylor cone and helps to control the fibers diameter. In this regard, low conductivity does not generate a charge at the droplet and no formation of Taylor cone, contrariwise, a good conductivity allows the cone formation, and fibers with small diameters $[68,69]$. However, when conductivity is higher than a critical value, ES process is not possible. The conductivity of polymer solution is influenced by the nature of polymer, the solvent or the addition of salts as conductive agents [70]. For AJS the 
conductivity of polymer solution has no influence on fiber production, due to the physical process is based on the strain of polymer by effect of air pressure.

\subsection{Environmental conditions}

ES and AJS can be conducted at room temperature and atmosphere condition, however, there is an influence of these environmental conditions in the final morphology of fibers, which can be controlled to obtain more uniform and reproducible fiber morphologies. Several studies have evaluated the effect of temperature and relative humidity on size and morphology of fibers.

a) Temperature

Temperature has two effects, allows the faster evaporation of solvents, meanwhile the viscosity of polymer solution decreases, hence, the diameter of fibers is reduced.

b) Relative humidity

Other studies clarify the effect of relative humidity with different polymers. In general, when humidity increases, the diameter was reduced, however if humidity is more than $70 \%$, beaded fibers are obtained. The presence of high hydrophilic polymers, as natural polymers, the presence of beads can be detected form $40 \%$ humidity [71]. The use of binary solvent systems and control of humidity may generate porous fibers. The different evaporation rates of the solvents used, where the more volatile solvent evaporated during the deposition generating a cooling effect, leading to condensation of water vapor. The water vapor forms droplets on the fibers, and mixed with the second solvent and evaporate, creating porous fibers. Thus, increasing when humidity is increased, the density pore density was higher, without change on the diameter [72].

\section{In vitro applications}

\subsection{Tissue engineering}


Tissue engineering is a multidisciplinary field, searching for biomaterials to provide three dimensional scaffolds to work as bioactive ECM and good mechanical properties to support cell growth and allow the generation of new tissues. Fiber scaffolds in micro- or nano- scale have been widely studied since it is possible to mimic the architecture of native extracellular matrix of tissues, besides have a microporous structure and high surface-area-to-volume ratio to facilitate the adhesion, proliferation and differentiation of cells $[27,73]$.

Electrical and non-electrical technologies are able to reproduce the fiber morphology of ECM and incorporate different molecules to reproduce the physical architecture and chemical composition of tissues. Natural and synthetic polymers have been processed by several electric and non-electric assisted technologies. The most used synthetic polymers are poly(lactic acid) (PLA), poly(glycolic acid) (PGA), their co-polymer PLGA, poly(caprolactone) (PCL), poly(ethylene oxide) (PEO). Synthetic polymers are biocompatible, biodegradable and have good mechanical properties, however, lack of bioactivity. Natural polymers as collagen, gelatin, elastin, silk fibroin, keratin and zein have been use for scaffold fabrication, due to their good biocompatibility, low immunogenicity and most of them have the inherent ability for binding cells through specific sequences as Arg-Gly-Asp (RGD) and Leu-Asp-Val (LDV) in their structure.

A broad of fiber scaffolds have been explored for bone tissue engineering. PCL electrospun fibers have shown to have stability to support cells along the time, thus, have been proposed as biomaterial for bone tissue engineering. Some results shown an increased cell proliferation and synthesis of extracellular matrix, characterized by collagen type I and mineralization [74]. Blended natural and synthetic polymers had gain attention to improve the biocompatibility and stability of biomaterials, as well the addition of inorganic materials for mineralized tissues or molecules to improve the bioactivity of fibers to guide tissue regeneration. Different natural polymers as collagen and gelatin have been blended with PCL to form fibers by electrospinning. [75,76]. Results shown an improvement in wettability and a better interaction with cells promoting bone osteogenesis. Proteins from vegetal sources have gain attention in their use for biomedical applications. Zein is the main 
component of endosperm corn. Zein fibers have been obtained by electrospinning and AJS, with a controllable morphology and diameter of fibers, by modifying process parameter. $[49,77,78]$. Moreover, zein fibers have shown to be water stable, with good mechanical properties. Zein has been used in combination with polymers as cellulose acetate to design electrospun fibers for tissue engineering applications or more specific with gelatin for periodontal[79,80]. Also, electrospun fibers of poly(glycerol sebacate) and zein have been explored for cardiac tissue engineering due to the improved mechanical and physicochemical properties by the addition of zein [81].

The understanding of hierarchical structure of bone, has led to the development of scaffolds composed by both, organic and inorganic components, to mimic the nanoscale composition of collagen fibers and hydroxyapatite (HA), respectively. Composites fibers produced by AJS have been widely explored as alternative for bone tissue engineering. Abdal-hay and cols. have been working in the design of different biocomposites PVA/hydroxyapatite, nHA/PLA fabricated by AJS [22,59]. Composites were suitable biomaterial for in vitro cell culture of osteoblast-like cells, with an appropriate osteoinductive signal and improvement of mechanical properties by presence of HA. Electrospun composite fibers have been widely explored by using different biomaterials. Electrospun fibers of gelatin modified with calcium phosphate and PCL to facilitate the cell interaction and mineralization [82]. Electrospinning fibers of PCL and HA which have been able to support cell growth by evaluating different stem cells, including bone marrow-derived mesenchymal stem cells (BMSCs), dental pulp stem cells (DPSCs) and adiposed-derived mesenchymal stem cells (ADSCs). Regarding to osteogenic differentiation, DPSCs showed higher calcium deposition, thus PCL/HA fiber scaffolds are a suitable material for bone tissue engineering [83]. Porous electrospun scaffolds of PCL, collagen I and nanoparticles of HA shown an increase of MSCs proliferation, with positive results for bone tissue engineering and allow the vascularization [84]. The good biocompatibility hybrid scaffolds and osteoinductive ability s due to the presence of collagen, the main protein present on ECM able to induce the nucleation for apatite. Meanwhile, synthetic polymers as PCL confer stability to the scaffolds along the time [85]. Electrospun fibers of silk fibroin for bone tissue 
engineering have been used due to the presence of RGD sequences specific for cell adhesion and used for the incorporation of HA nanoparticles [86]. Hydroxyapatite nanoparticles have been incorporated in silk fibroin electrospun fibers without modified the size and morphology of fibers and improving the mechanical properties of fibers. A post-treatment of these composite fibers, can be achieved to promote osteogenic differentiation through the use of growth factors as bone morphogenetic protein2 (BMP-2) [87]. As well, polysaccharides have been considered as biomaterials. Chitosan is the most used for tissue engineering and as drug carrier for different molecules [88]. Chitosan fibers have been prepared by electrospinning and treated to enhance the formation of HA [89]. Results shown that cell proliferation increased and enhanced the cell differentiation, due to the fiber structure and composition. Chitosan nanofibers with PVA were fabricated by AJS to form three-dimensional mats that are highly hydrophilic and can form an hydrogel, suggested to use as dressing for wound healing [90].

The arrangement of fibers is an important factor for muscle, tendons, periodontal ligament and nerve regeneration. Electrospinning and AJS have been use for the develop of aligned fibers from different biomaterials $[24,25,91,92]$. For instance, cells seeded onto PCL/gelatin aligned electrospun fibers shown a preferential direction in their morphology and the extent elongation of neurite along the fibers [93]. A similar cell behavior was reported in electrospun fibers of PCL and elastin fibers [94]. The elongated shape of cardiac cell lines was investigated onto PLA and polyurethane aligned fibers produced by AJS [36]. The morphology of cardiac cell lines was highly influenced by fiber orientation, resulting in a similar phenotype than in living tissues.

Titanium materials have been widely used as implantable material. However, is a bioinert material, thus, is not able to interact with cells and create a functional interface with surrounding tissues. For this reason, coating strategies are required to improve the interaction of these materials with cells. Electro and non-electro assisted technologies can be use also for coating surfaces. PVA was used to coat titanium surfaces via AJS and evaluated in vitro the effect of coated surfaces in presence of MC3T3-E1 pre-osteoblast cell line. The results showed a highly interconnected porous structure over 
Ti surface which allowed the cell adhesion and improve bioactivity [31]. There are several studies on the use of AJS to fabricate hybrid nanofibers composed by nanohydroxyapatite and PLA, and poly(vinyl acetate) (PVA) with hydroxyapatite. Hydroxyapatite was greatly incorporated into the fibers no providing change in fiber morphology or size and have been used as coating for Ti surfaces. [22,31,59]. Titanium surfaces have been coated with natural proteins as keratin to improve the cell

adhesion of fibroblast for dental implant applications by electrospinning process [95]. Moreover, aligned keratin electrospun fibers deposited onto these Ti surfaces allow the alignment of fibroblast along the fibers, similar to periodontal ligament arrangement in nature [96]. As periodontal ligament, the particular morphology of nerve, musculoskeletal and cardiac muscle tissues makes necessary to control the arrangement of fibers. In this regard, PLA and PU fibers have been fabricate by AJS.

\subsection{Molecular delivery}

In tissue engineering concerning the regeneration of wound or damage tissue and in several treatment for bacterial infections, the most common administration of growth factor, biomolecules or drugs is by enteral routes, in the form of tablets, capsules, granules, etc., while some are administered by parenteral routes, such as intravenous, intra-arterial, intramuscular, or subcutaneous. However, this kind of administration have several disadvantages, such as first-pass metabolism, discomfort, pain and in bacterial treatment is more challenging due to the development of new resistance mechanisms during drug administration [97]. Thus, the research is mainly focused on new novelty systems for delivery directly in the place where the biological action is needed to carry out. For this purpose, electro- and non- electro assisted spinning may be considered versatile techniques to fabricate microand nanofibers able to incorporate hydrophilic drugs, growth factors, biomolecules, proteins or peptides, for a sustained delivery and controlled burst effects that is gaining a tremendous success in recent approaches for cancer therapy, nanomedicine and disease diagnosis [97,98]. Moreover, molecular delivery systems fabricated via electro- and non- electro assisted spinning techniques show high loading capacity, high encapsulation efficiency, ease of operation and cost-effectiveness, due to 
the high surface-to-volume ratio of fibers/particles, concurring to accelerate the solubility of the drug in the aqueous solution and improving the efficiency of the drug. In this context, the peculiar properties of biodegradable polymers may concur to protect encapsulated biomolecules or drugs from corrosion of gastric acid and enzymes, maintaining the bioactivity of the material [99].

The successful of molecular delivery by the electro- and non- electro assisted spinning depends of the bioactive molecule desirable to be loaded. The most research on this field involve the electrospinning device system because allow to carefully choose the polymeric or composite system for the preservation of the therapeutic effect, for example blending electrospinning method where drug encapsulation is achieved through electrospinning in a single step, because drugs are dissolved or dispersed in the polymeric solution [100,101]. In coaxial electrospinning is used for the production of core-shell nanofibers that give protection to the loaded compound and is used to obtained fibers with specific drugs encapsulated in the core of the fibers, which lead to a sustained and controlled drug release [102,103]. In emulsion electrospinning involve the mixtures of two or more immiscible liquids, where one liquid is usually dispersed as drops in the other, which is seen as a continuous phase, so the biomolecule or drug phase has a sufficient low molecular weight that allowing to load and obtain a well distribution within the fibre [103,104].

Numerous studies have reported the development of nanofiber spun mats for molecular delivery applications with reported successfully activity in the biomedical field. The antibiotics and antibacterial agents have been the most common drug molecules encapsulated, using different polymers such as PLA, PLGA and PCL for its biodegradability and also used for controlling the release pattern of the drug. In our group, explore the use of the integrated electrofluidodynamics (EFDs) technology combining electrospinning and electrospraying for designing nanostructured platforms with controlled release to prevent the formation of bacterial biofilms in oral implant sites. The results of this strategies were that combining this technology allow to be synthetized polycaprolactone (PCL) nanofibers decorated with chitosan (CS) nanoparticles at the same time, giving a more efficacious systems in terms of degradation protection, pharmacokinetic control and 
drug. Moreover, the hydrophobic properties of the PCL network promote a more homogeneous spatial distribution of nanoreservoirs for the amoxicillin trihydrate (AMX-DTH) and tetracycline hydrochloride $(\mathrm{TCH})$, improving the activity against bacteria by a more efficient drug confinement and serves as an innovative antibacterial treatment. The antibacterial properties were evaluated by halo inhibition zone size of the bacterial onto agar plates showing that CS/PCL integrated platforms of (AMX-DTH and TCH) showed a food antibacterial response against of three different population of bacteria as $S$. aureus, E. coli and A. actinomycetemcomitans. The results showed that fabricated platforms, could open new innovative routes for multiple drug release, as more effective therapies to overcome the limitation of the conventional antibiotic therapies by systemic administration in the presence of periodontal diseases where concerns the low efficacy to fight bacteria attacks during long treatment times [105].

In skin tissue engineering the fabrication of a skin grafts or analogues frequently play an important role in the treatment of chronic skin wounds, by supporting the regeneration of newly formed tissue, and at the same time preventing infections during the long-term treatment. In our study we focused on use the collagen as mimicking the structural protein of the dermal tissue combine with the properties of PCL for the fabrication of a micro/nano-structured matrices where the encapsulation of drugs, such as gentamicin sulfate also was explored as the capability for loaded into collagen-added nanofibers, for the controlled release in local infection treatments. The results showed that collagen added fibers can be efficaciously used to administrate gentamicin for $72 \mathrm{~h}$, improves the bioactivity of nanofibers and not showed any cytotoxicity when culture onto human dermal fibroblast after 5 days indicating that composite of PCL/Col serves as molecular delivery platform with good potential in skin tissue regeneration [16].

Concerning the treatment of pain and inflammation associated with rheumatoid arthritis, osteoarthritis, the drug most used are the non-steroidal anti-inflammatory analgesics (NSAIDs) for try to relief of moderate pain. In biomedical field the search on new molecular delivery for this application are focused on the fabrication of capsules or particles with peculiar properties (e.g., 
swelling, $\mathrm{pH}$-sensitive response) at the micro and sub-micrometric size scale, to be used as carriers for controlled drug and molecular release. In our group, special synthesis using electrohydrodynamic atomization an electro-dropping technology was able to development a mono-component device made of cellulose acetate based on the use of coaxial needles to design core/shell architectures to confine anti-inflammatory drugs (ketoprofen lysine) as microcarriers with mono- (MC) or bi-phasic (BC) composition as more efficiently for oral delivery applications. The results showed that design bi-phasic CA capsules improved encapsulation and release properties, in comparison with monophasic ones, by imparting a core shell structure to the device and this system could be a very promising for developing versatile delivery systems for a sustained molecular release of therapeutic agents in oral treatments [106].

The large variability of material properties and the high sensitivity of non-electro and electro-assisted technologies gives the opportunity to design nanomaterials for targeting. In particular, recent discoveries have addressed their use for diagnosis also in combination with therapeutic treatments namely theranostics [107].

For instance, electrospinning has been recently used to fabricate composite nanofibers including magnetic particles such as $\mathrm{Fe}_{3} \mathrm{O}_{4}$. The application of electrostatic forces do not alter the magnetic properties of nanoparticles, homogeneously distributed into the fibres, thus making them suitable as magnetic drug delivery systems in theranostics applications [108].

Core-shell fibers have been recently designed by coaxial electrospinning to produce fibers at the nanometric size scale with image contrast properties. In particular, these fibres are able to retain the compounds - working as contrast agents - in the core of fibers, and transferring them directly onto the colon mucosa for the selected targeting of the tissue, by a controlled degradation of the shell [109] . In this case, the further addition of therapeutic drugs has been considered to design a sustainable release of drugs driven by an in situ mechanism of matrix erosion/ drug diffusion [110], for the therapeutic treatment of the targeted tissue.. 
Similarly, coaxial electrospray is emerging as a successful strategy for the development of multimodal particles with potential applications teragnostic by a combined approach based on imaging and therapy [111].

For instance, multishell particles have been recently engineered by the implementation of tri-needle coaxial system. This technology allowed in a single-step to form polymer-based magnetic yolk-shell particles for multifunctional theragnostic agents for dual-imaging modality and magnetically controlled coactive delivery [112]. Likewise, coaxial electrospray has been used to produce theragnostic lipoplexes for imaging and therapeutic functions. This kind of systems may efficiently use to load drugs and contrast agents, thus reducing the toxicity effects and improving the therapeutic efficacy by the enhancement of the circulation time [113].

\section{In vivo models}

In bone tissue engineering the most common therapy to achieve bone reconstruction or function is based on grafts (autografts, xenografts, and allografts), or the implantation of metal devices or ceramic-based implants, which serve as support matrix, filler and/or stabilizers for allow the regeneration of the tissue. All these grafts strategies have limited in access and availability and some grafts harvest is associated with donor site morbidity, haemorrhage, risk of infection, insufficient transplant integration, and graft devitalization [114]. In tissue engineering, the electro- and nonelectro assisted spinning is emerged as a process technology suitable to precisely design the architecture of the micro- and nano- scale scaffolds with controlled pore size and interconnectivity, able to mimic the morphological characteristics of the native extracellular matrix in association with cells and/or growth factors to produce implantable scaffold for in vitro regeneration. However, for recreated the in vivo environment of human tissue and evaluated if the designed scaffold by the electro- and non- electro assisted spinning could regenerated the complex structure of critical size 
defects of bone, cartilage, nerves, vasculature, and soft tissues, various animal models have been developed [115-117].

The term related to "critical size defect" has been originally defined as "the smallest size intra-osseous wound in a particular bone and species that will not heal spontaneously during the lifetime of the animal" [118]. However, there are various parameters for establish the critical size defect and the most important is related to no mineralized area of $\geq 30 \%$ after 52 weeks, and bone deficiency whose length exceed 2-2.5 times the diameter of the affected bone and where there will be no a complete bony regeneration [119]. The selection of the animal model must be in agreement to observe in time the regeneration of the tissue and the most common models for the evaluation of tissues defects have been mice, rats, rabbits, dogs, goat, sheep and pigs [120-123]. Nevertheless, various factors must be considered for selecting a specific animal species as a testing model. One of the principle factors are physiological and pathophysiological analogies with humans, as Gomes says "The selection of preclinical models often takes the phylogenetic tree into consideration; however, if can be achieved using small animals, like rodents, it is preferable" [124]. Regarding bone regeneration, rodents may present several advantages, such as a better cost-effectiveness ratio, easier housing and manageable to operate, and allow standardization of experimental conditions in genetically similar individuals and observe a multiplicity of study objects post-surgery over a relative short period time $[119,125]$. There are different bone defect sites being used to evaluate bone graft substitutes, but the main are the calvaria defect, femur or segmental defect, ulna, partial cortical defect and cancellous bone defect models. The segmental and calvarial bone defects are the most widely described and used in the literature. Moreover, the calvarial defect model has been widely used for the following reasons: the calvarial bone is a standardized defect, which allows creation of a uniform circular defect, using a trephine bur with saline irrigation, and the excised bone disk is removed to prevent damage to the dura mater. Furthermore, concerning the scaffold to be implanted does not need any fixation because dura mater serves as support as well as the overlying skin; and permits precise comparison of a variety 
of scaffold; and enables radiographical and histological analyses. Although segmental bone defects in long bones could be used to more closely mimic the clinical scenario [126-128].

Several investigations have been conducted on calvarial healing in rats with defect sizes measuring from 5 to $8 \mathrm{~mm}$ in diameter and this critical defect allow to determine whether the spun material has biological properties as osteoconductive, osteoinductive and facilitates bone regeneration. Some studies using the fabrication of fibre spun mats by the electro- and non- electro assisted spinning have been explore the repair of non-union critical-sized bone defects because fibers scaffolds have showed excellent response in bone applications in vitro and open the possibility for being good candidates for rebuilding osteogenic ECM microenvironment in vivo. Li et al., prepared a modified biomimetic gelatin/hydroxyapatite nanofibrous scaffolds by electrospinning and reporting that the scaffold has good biocompatibility, expression of bone markers by mesenchymal stem cells and when the scaffold were implanted in a rat calvarial defect model could serve as a template for guiding bone regeneration and the bone defects were almost repaired completely $(94.28 \% \pm 5.00 \%)$ at 6 weeks post implanted [129].

Zhang et al., investigated the aligned electrospun cellulose/CNCs nanocomposite nanofibers (ECCNNs) loaded with bone morphogenic protein-2 (BMP-2) could mimicking the ECM structure to recruit stem cells in vivo and the result analysed by micro CT evaluation showed that after 12 weeks had much bone formation and the volume of the newly formed bone volume $(8.63 \mathrm{~mm} 3)$ and bone mineral density $(14.09 \mathrm{~g} / \mathrm{cm} 3)$ were significantly higher when compared to those for the defectonly group. Moreover, the histology analyses showed new bone formation and the nanofibers were completely covered by newly formed aligned collagen fibers, which were integrated into the host bone tissues [130].

Han et al., explored the mineralized electrospun polylactic acid (PLLA) nanofibrous membranes containing different amounts of strontium $(\mathrm{Sr})$ fabricated by an electrodeposition method in cranial bone defect experiments and at 8 weeks post-implantation, micro-CT analysis revealed that new bone formation was stronger with increasing $\mathrm{Sr}$ content and supported by histological analysis stained with 
Van Gieson picrofuchsin clearly showed that nanofibrous membranes could significantly enhance newly bone formation [131].

Yao et al., synthesized a 3D electrospun PCL/PLA blend nanofibrous scaffolds and explore in a clinically relevant critical-size cranial bone defect mouse model if the proposed scaffold has the ability to allow in vivo bone formation for up to 6 weeks with and without the presence of BMP growth factor. The results reported that all PCL/PLA-rhBMP2 group exhibited some new bony tissue formation and the scaffold would provide a more favourable/desired microenvironment for mouse cranial bone formation as compared to the previously reported PCL-3D scaffold [13].

All these results suggested that calvarial defect model will be utilized to deeply understand all the regeneration processes involving fibrous scaffolds with osteoinductive or conductive response in the presence of progenitor cells, growth factors, or epigenetic instructions, towards the use of electroand non- electro assisted spinning technologies for a translational medicine addressed to the clinical problems of patients.

\section{Acknowledgments}

Financial support of PAPIIT and IT203618 


\section{References}

[1] V. Guarino, L. Ambrosio, Electrofluidodynamics: exploring a new toolbox to design biomaterials for tissue regeneration and degeneration, Nanomedicine. 11 (2016) 1515-1518. doi:10.2217/nnm-2016-0108.

[2] A. Abdal-Hay, N.M. Barakat, J.K. Lim, Novel Technique for Polymeric Nanofibers Preparation: Air Jet Spinning, Sci. Adv. Mater. 4 (2012) 1268-1275. doi:10.1166/sam.2012.1382.

[3] V. Guarino, V. Cirillo, R. Altobelli, L. Ambrosio, Polymer-based platforms by electric fieldassisted techniques for tissue engineering and cancer therapy., Expert Rev. Med. Devices. 12 (2015) 113-29. doi:10.1586/17434440.2014.953058.

[4] V. Guarino, V. Cirillo, L. Ambrosio, Bicomponent electrospun scaffolds to design extracellular matrix tissue analogs, 2016. doi:10.1586/17434440.2016.1126505.

[5] R. Altobelli, V. Guarino, L. Ambrosio, Micro- and nanocarriers by electrofludodynamic technologies for cell and molecular therapies, Process Biochem. 51 (2016) 2143-2154. doi:10.1016/j.procbio.2016.09.002.

[6] V. Guarino, R. Altobelli, V. Cirillo, A. Cummaro, L. Ambrosio, Additive electrospraying: a route to process electrospun scaffolds for controlled molecular release, Polym. Adv. Technol. 26 (2015) 1359-1369. doi:10.1002/pat.3588.

[7] V. Guarino, I. Cruz-Maya, R. Altobelli, W.K. Abdul Khodir, L. Ambrosio, M.A. AlvarezPerez, A. Almaguer-Flores, Electrospun polycaprolactone nanofibres decorated by drug loaded chitosan nano-reservoirs for antibacterial treatments, Nanotechnology. 28 (2017) 505103. doi:10.1088/1361-6528/aa9542.

[8] P.D. Dalton, C. Vaquette, B.L. Farrugia, T.R. Dargaville, T.D. Brown, D.W. Hutmacher, Electrospinning and additive manufacturing: converging technologies, Biomater. Sci. 1 (2013) 171-185. doi:10.1039/C2BM00039C.

[9] Y. Il Yoon, K.E. Park, S.J. Lee, W.H. Park, Fabrication of microfibrous and nano- 
/microfibrous scaffolds: Melt and hybrid electrospinning and surface modification of poly(Llactic acid) with plasticizer, Biomed Res. Int. 2013 (2013). doi:10.1155/2013/309048.

[10] H. Lian, Z. Meng, Melt electrospinning vs. solution electrospinning: A comparative study of drug-loaded poly ( $\varepsilon$-caprolactone) fibres, Mater. Sci. Eng. C. 74 (2017) 117-123. doi:10.1016/j.msec.2017.02.024.

[11] A.A. Bulysheva, G.L. Bowlin, A.J. Klingelhutz, W.A. Yeudall, Low-temperature electrospun silk scaffold for in vitro mucosal modeling, J. Biomed. Mater. Res. Part A. 100A (2012) 757-767. doi:10.1002/jbm.a.33288.

[12] T. Xu, J.M. Miszuk, Y. Zhao, H. Sun, H. Fong, Electrospun Polycaprolactone 3D Nanofibrous Scaffold with Interconnected and Hierarchically Structured Pores for Bone Tissue Engineering, Adv. Healthc. Mater. 4 (2015) 2238-2246. doi:10.1002/adhm.201500345.

[13] Q. Yao, J.G.L. Cosme, T. Xu, J.M. Miszuk, P.H.S. Picciani, H. Fong, H. Sun, Three dimensional electrospun PCL/PLA blend nanofibrous scaffolds with significantly improved stem cells osteogenic differentiation and cranial bone formation, Biomaterials. 115 (2017) 115-127. doi:10.1016/j.biomaterials.2016.11.018.

[14] T.J. Sill, H.A. von Recum, Electrospinning: Applications in drug delivery and tissue engineering, Biomaterials. 29 (2008) 1989-2006. doi:10.1016/j.biomaterials.2008.01.011.

[15] K. Garg, G.L. Bowlin, Electrospinning jets and nanofibrous structures, Biomicrofluidics. 5 (2011) 013403. doi:10.1063/1.3567097.

[16] W. Abdul Khodir, A. Abdul Razak, M. Ng, V. Guarino, D. Susanti, Encapsulation and Characterization of Gentamicin Sulfate in the Collagen Added Electrospun Nanofibers for Skin Regeneration, J. Funct. Biomater. 9 (2018) 36. doi:10.3390/jfb9020036.

[17] J. Yan, L. Qiang, Y. Gao, X. Cui, H. Zhou, S. Zhong, Q. Wang, H. Wang, Effect of fiber alignment in electrospun scaffolds on keratocytes and corneal epithelial cells behavior, J. Biomed. Mater. Res. - Part A. 100 A (2012) 527-535. doi:10.1002/jbm.a.33301. 
[18] A. Abdal-Hay, A.S. Hamdy, J.H. Lim, Facile preparation of titanium dioxide micro/nanofibers and tubular structures by air jet spinning, Ceram. Int. 40 (2014) 1540315409. doi:10.1016/j.ceramint.2014.06.028.

[19] S. François, C. Sarra-Bournet, A. Jaffre, N. Chakfé, B. Durand, G. Laroche, Characterization of an air-spun poly(L-lactic acid) nanofiber mesh, J. Biomed. Mater. Res. - Part B Appl. Biomater. 93 (2010) 531-543. doi:10.1002/jbm.b.31612.

[20] R.E. Benavides, S.C. Jana, D.H. Reneker, Nanofibers from scalable gas jet process, ACS Macro Lett. 1 (2012) 1032-1036. doi:10.1021/mz300297g.

[21] J.L. Daristotle, A.M. Behrens, A.D. Sandler, P. Kofinas, A Review of the Fundamental Principles and Applications of Solution Blow Spinning, ACS Appl. Mater. Interfaces. 8 (2016) 34951-34963. doi:10.1021/acsami.6b12994.

[22] A. Abdal-Hay, A.S. Hamdy, K.A. Khalil, J.H. Lim, A novel simple one-step air jet spinning approach for deposition of poly(vinyl acetate)/hydroxyapatite composite nanofibers on $\mathrm{Ti}$ implants, Mater. Sci. Eng. C. 49 (2015) 681-690. doi:10.1016/j.msec.2015.01.008.

[23] E.J. Kuk, M.G. Hwang, M.G. Kim, S.B. Kim, Y.J. Kim, J.K. Lim, PCL / PEO composite Nanofiber Manufacturing Technology using Air Jet Spinning method, (2014). doi:10.15242/iie.e0214517.

[24] M.M.O. Simbara, A.R. Santos, A.J.P. Andrade, S.M. Malmonge, Comparative study of aligned and nonaligned poly( $\varepsilon$-caprolactone) fibrous scaffolds prepared by solution blow spinning, J. Biomed. Mater. Res. Part B Appl. Biomater. 107 (2019) 1462-1470. doi:10.1002/jbm.b.34238.

[25] A. Magaz, A.D. Roberts, S. Faraji, T.R.L. Nascimento, E.S. Medeiros, W. Zhang, R.D. Greenhalgh, A. Mautner, X. Li, J.J. Blaker, Porous, Aligned and Biomimetic Fibers of Regenerated Silk Fibroin Produced by Solution Blow Spinning, Biomacromolecules. (2018) acs.biomac.8b01233. doi:10.1021/acs.biomac.8b01233.

[26] E. Hofmann, K. Krüger, C. Haynl, T. Scheibel, M. Trebbin, S. Förster, Microfluidic nozzle 
device for ultrafine fiber solution blow spinning with precise diameter control, Lab Chip. 18 (2018) 2225-2234. doi:10.1039/c81c00304a.

[27] J.L. Suarez-Franco, F.C. Vázquez-Vázquez, A. Pozos-Guillen, J.J. Montesinos, O. AlvarezFregoso, M.A. Alvarez-Perez, Influence of diameter of fiber membrane scaffolds on the biocompatibility of hpdl mesenchymal stromal cells, Dent. Mater. J. 37 (2018) 465-473. doi:10.4012/dmj.2016-329.

[28] M.V. Granados-Hernández, J. Serrano-Bello, J.J. Montesinos, C. Alvarez-Gayosso, L.A. Medina-Velázquez, O. Alvarez-Fregoso, M.A. Alvarez-Perez, In vitro and in vivo biological characterization of poly(lactic acid) fiber scaffolds synthesized by air jet spinning, J. Biomed. Mater. Res. - Part B Appl. Biomater. 106 (2018) 2435-2446. doi:10.1002/jbm.b.34053.

[29] R.T. Paschoalin, B. Traldi, G. Aydin, J.E. Oliveira, S. Rütten, L.H.C. Mattoso, M. Zenke, A. Sechi, Solution blow spinning fibres: New immunologically inert substrates for the analysis of cell adhesion and motility, Acta Biomater. 51 (2017) 161-174. doi:10.1016/j.actbio.2017.01.020.

[30] A. Ali, A. Valencia, N.V.B. Phd, F. Carolina, V. Vázquez, T.V. K, J. José, M. Montesinos, E.A.M. Msc, M. Antonio, Á. Pérez, R.D.P. De Prostodoncia, U.D.C. Rica, C. Rica, Biocompatibilidad de andamios nanofibrilares con diferentes concentraciones de PLA / Hidroxiapatita Biocompatibility of Nanofibrous Scaffolds with Different Concentrations of PLA / Hydroxyapatite, 3 (2016) 51-62.

[31] A. Abdal-Hay, A.S. Hamdy, M.Y. Abdellah, J. Lim, In vitro bioactivity of implantable Ti materials coated with PVAc membrane layer, Mater. Lett. 126 (2014) 267-270. doi:10.1016/j.matlet.2014.04.048.

[32] W. Tutak, S. Sarkar, S. Lin-Gibson, T.M. Farooque, G. Jyotsnendu, D. Wang, J. Kohn, D. Bolikal, C.G. Simon, The support of bone marrow stromal cell differentiation by airbrushed nanofiber scaffolds, Biomaterials. 34 (2013) 2389-2398. doi:10.1016/j.biomaterials.2012.12.020. 
[33] F.C. Vazquez-Vazquez, O.A. Chanes-Cuevas, D. Masuoka, J.A. Alatorre, D. ChavarriaBolaños, J.R. Vega-Baudrit, J. Serrano-Bello, M.A. Alvarez-Perez, Biocompatibility of Developing 3D-Printed Tubular Scaffold Coated with Nanofibers for Bone Applications, J. Nanomater. 2019 (2019) 1-13. doi:10.1155/2019/6105818.

[34] J.L. Daristotle, S.T. Zaki, L.W. Lau, L. Torres, A. Zografos, P. Srinivasan, O.B. Ayyub, A.D. Sandler, P. Kofinas, Improving the adhesion, flexibility, and hemostatic efficacy of a sprayable polymer blend surgical sealant by incorporating silica particles, Acta Biomater. 90 (2019) 205-216. doi:10.1016/j.actbio.2019.04.015.

[35] C.L. Miller, G. Stafford, N. Sigmon, J.A. Gilmore, Conductive nonwoven carbon nanotubePLA composite nanofibers towards wound sensors via solution blow spinning, IEEE Trans. Nanobioscience. 18 (2019) 244-247. doi:10.1109/TNB.2019.2905507.

[36] E. Tomecka, M. Wojasinski, E. Jastrzebska, M. Chudy, T. Ciach, Z. Brzozka, Poly(L-lactic acid) and polyurethane nanofibers fabricated by solution blow spinning as potential substrates for cardiac cell culture, Mater. Sci. Eng. C. 75 (2017) 305-316. doi:10.1016/j.msec.2017.02.055.

[37] D.R. Bienek, K.M. Hoffman, W. Tutak, Blow-spun chitosan/PEG/PLGA nanofibers as a novel tissue engineering scaffold with antibacterial properties, J. Mater. Sci. Mater. Med. 27 (2016) 0-1. doi:10.1007/s10856-016-5757-7.

[38] X. Zhuang, X. Yang, L. Shi, B. Cheng, K. Guan, W. Kang, Solution blowing of submicronscale cellulose fibers, Carbohydr. Polym. 90 (2012) 982-987. doi:10.1016/j.carbpol.2012.06.031.

[39] M.A. Souza, J.E. Oliveira, E.S. Medeiros, G.M. Glenn, L.H.C. Mattoso, Controlled Release of Linalool Using Nanofibrous Membranes of Poly(lactic acid) Obtained by Electrospinning and Solution Blow Spinning: A Comparative Study, J. Nanosci. Nanotechnol. 15 (2015) 5628-5636. doi:10.1166/jnn.2015.9692.

[40] W. Tutak, G. Gelven, C. Markle, X. Palmer, Rapid polymer fiber airbrushing: Impact of a 
device design on the fiber fabrication and matrix quality, J. Appl. Polym. Sci. 132 (2015) n/a-n/a. doi:10.1002/app.42813.

[41] E.S. Medeiros, G.M. Glenn, A.P. Klamczynski, W.J. Orts, L.H.C. Mattoso, Solution blow spinning: A new method to produce micro- and nanofibers from polymer solutions, J. Appl. Polym. Sci. 113 (2009) 2322-2330. doi:10.1002/app.30275.

[42] J.. Deitzel, J. Kleinmeyer, D. Harris, N.. Beck Tan, The effect of processing variables on the morphology of electrospun nanofibers and textiles, Polymer (Guildf). 42 (2001) 261-272. doi:10.1016/S0032-3861(00)00250-0.

[43] T. Volova, D. Goncharov, A. Sukovatyi, A. Shabanov, E. Nikolaeva, E. Shishatskaya, Electrospinning of polyhydroxyalkanoate fibrous scaffolds: effects on electrospinning parameters on structure and properties., J. Biomater. Sci. Polym. Ed. 25 (2014) 370-93. doi:10.1080/09205063.2013.862400.

[44] F. Liu, R. Guo, M. Shen, S. Wang, X. Shi, Effect of Processing Variables on the Morphology of Electrospun Poly[(lactic acid)- co -(glycolic acid)] Nanofibers, Macromol. Mater. Eng. 294 (2009) 666-672. doi:10.1002/mame.200900110.

[45] S. Huan, G. Liu, G. Han, W. Cheng, Z. Fu, Q. Wu, Q. Wang, Effect of Experimental Parameters on Morphological, Mechanical and Hydrophobic Properties of Electrospun Polystyrene Fibers, Materials (Basel). 8 (2015) 2718-2734. doi:10.3390/ma8052718.

[46] F. Topuz, T. Uyar, Electrospinning of gelatin with tunable fiber morphology from round to flat/ribbon, Mater. Sci. Eng. C. 80 (2017) 371-378. doi:10.1016/j.msec.2017.06.001.

[47] D.D. da Silva Parize, M.M. Foschini, J.E. de Oliveira, A.P. Klamczynski, G.M. Glenn, J.M. Marconcini, L.H.C. Mattoso, Solution blow spinning: Parameters optimization and effects on the properties of nanofibers from poly(lactic acid)/dimethyl carbonate solutions, J. Mater. Sci. 51 (2016) 4627-4638. doi:10.1007/s10853-016-9778-x.

[48] C. Wang, C.H. Hsu, J.H. Lin, Scaling laws in electrospinning of polystyrene solutions, Macromolecules. 39 (2006) 7662-7672. doi:10.1021/ma060866a. 
[49] F. Liu, R.J. Avena-Bustillos, R. Woods, B. Sen Chiou, T.G. Williams, D.F. Wood, C. Bilbao-Sainz, W. Yokoyama, G.M. Glenn, T.H. McHugh, F. Zhong, Preparation of Zein Fibers Using Solution Blow Spinning Method, J. Food Sci. 81 (2016) N3015-N3025. doi:10.1111/1750-3841.13537.

[50] X. Geng, O.H. Kwon, J. Jang, Electrospinning of chitosan dissolved in concentrated acetic acid solution, Biomaterials. 26 (2005) 5427-5432. doi:10.1016/j.biomaterials.2005.01.066.

[51] A.H. Hekmati, A. Rashidi, R. Ghazisaeidi, J.Y. Drean, Effect of needle length, electrospinning distance, and solution concentration on morphological properties of polyamide-6 electrospun nanowebs, Text. Res. J. 83 (2013) 1452-1466. doi:10.1177/0040517512471746.

[52] C. Zhang, X. Yuan, L. Wu, Y. Han, J. Sheng, Study on morphology of electrospun poly(vinyl alcohol) mats, Eur. Polym. J. 41 (2005) 423-432. doi:10.1016/j.eurpolymj.2004.10.027.

[53] S. Megelski, J.S. Stephens, D.B. Chase, J.F. Rabolt, Micro- and Nanostructured Surface Morphology on Electrospun Polymer Fibers, Macromolecules. 35 (2002) 8456-8466. doi:10.1021/ma020444a.

[54] I. Cruz Maya, V. Guarino, Introduction to electrofluidodynamic techniques. Part I, in: V. Guarino, L.B.T.-E.T. (EFDTs) for B. and M.D. Ambrosio (Eds.), Electrofluidodynamic Technol. Biomater. Med. Devices, Elsevier, 2018: pp. 1-17. doi:10.1016/B978-0-08-101745$6.00001-3$

[55] S.A. Theron, E. Zussman, A.L. Yarin, Experimental investigation of the governing parameters in the electrospinning of polymer solutions, Polymer (Guildf). 45 (2004) 20172030. doi:10.1016/j.polymer.2004.01.024.

[56] S. Zargham, S. Bazgir, A. Tavakoli, A.S. Rashidi, R. Damerchely, The Effect of Flow Rate on Morphology and Deposition Area of Electrospun Nylon 6 Nanofiber, J. Eng. Fiber. Fabr. 7 (2012) 155892501200700. doi:10.1177/155892501200700414. 
[57] W. Zuo, M. Zhu, W. Yang, H. Yu, Y. Chen, Y. Zhang, Experimental study on relationship between jet instability and formation of beaded fibers during electrospinning, Polym. Eng. Sci. 45 (2005) 704-709. doi:10.1002/pen.20304.

[58] J.E. Oliveira, E.A. Moraes, R.G.F. Costa, A.S. Afonso, L.H.C. Mattoso, W.J. Orts, E.S. Medeiros, Nano and submicrometric fibers of poly(D,L-lactide) obtained by solution blow spinning: Process and solution variables, J. Appl. Polym. Sci. 122 (2011) 3396-3405. doi:10.1002/app.34410.

[59] A. Abdal-hay, F.A. Sheikh, J.K. Lim, Air jet spinning of hydroxyapatite/poly(lactic acid) hybrid nanocomposite membrane mats for bone tissue engineering, Colloids Surfaces B Biointerfaces. 102 (2013) 635-643. doi:10.1016/j.colsurfb.2012.09.017.

[60] T. Wang, S. Kumar, Electrospinning of polyacrylonitrile nanofibers, J. Appl. Polym. Sci. 102 (2006) 1023-1029. doi:10.1002/app.24123.

[61] P. Gupta, C. Elkins, T.E. Long, G.L. Wilkes, Electrospinning of linear homopolymers of poly(methyl methacrylate): Exploring relationships between fiber formation, viscosity, molecular weight and concentration in a good solvent, Polymer (Guildf). 46 (2005) 47994810. doi:10.1016/j.polymer.2005.04.021.

[62] S. François, N. Chakfé, B. Durand, G. Laroche, A poly(l-lactic acid) nanofibre mesh scaffold for endothelial cells on vascular prostheses, Acta Biomater. 5 (2009) 2418-2428. doi:10.1016/j.actbio.2009.03.013.

[63] B. Tarus, N. Fadel, A. Al-Oufy, M. El-Messiry, Effect of polymer concentration on the morphology and mechanical characteristics of electrospun cellulose acetate and poly (vinyl chloride) nanofiber mats, Alexandria Eng. J. 55 (2016) 2975-2984. doi:10.1016/j.aej.2016.04.025.

[64] H. Fong, I. Chun, D.H. Reneker, Beaded nanofibers formed during electrospinning, in: Polymer (Guildf)., 1999: pp. 4585-4592. doi:10.1016/S0032-3861(99)00068-3.

[65] X. Zong, K. Kim, D. Fang, S. Ran, B.S. Hsiao, B. Chu, Structure and process relationship of 
electrospun bioabsorbable nanofiber membranes, Polymer (Guildf). 43 (2002) 4403-4412. doi:10.1016/S0032-3861(02)00275-6.

[66] C. Bilbao-Sainz, B. Sen Chiou, D. Valenzuela-Medina, W.X. Du, K.S. Gregorski, T.G. Williams, D.F. Wood, G.M. Glenn, W.J. Orts, Solution blow spun poly(lactic acid)/hydroxypropyl methylcellulose nanofibers with antimicrobial properties, Eur. Polym. J. 54 (2014) 1-10. doi:10.1016/j.eurpolymj.2014.02.004.

[67] S. Tungprapa, T. Puangparn, M. Weerasombut, I. Jangchud, P. Fakum, S. Semongkhol, C. Meechaisue, P. Supaphol, Electrospun cellulose acetate fibers: Effect of solvent system on morphology and fiber diameter, Cellulose. 14 (2007) 563-575. doi:10.1007/s10570-0079113-4.

[68] C.J. Angammana, Thesis: A Study of the Effects of Solution and Process Parameters on the Electrospinning Process and Nanofibre Morphology, 47 (2011) 1109-1117.

[69] V. Guarino, V. Cirillo, P. Taddei, M.A. Alvarez-Perez, L. Ambrosio, Tuning size scale and crystallinity of PCL electrospun fibres via solvent permittivity to address hMSC response, Macromol. Biosci. 11 (2011) 1694-1705. doi:10.1002/mabi.201100204.

[70] R.P.A. Hartman, D.J. Brunner, D.M.A. Camelot, J.C.M. Marijnissen, B. Scarlett, Jet breakup in electrohydrodynamic atomization in the cone-jet mode, J. Aerosol Sci. 31 (2000) 6595. doi:10.1016/S0021-8502(99)00034-8.

[71] J. Pelipenko, J. Kristl, B. Janković, S. Baumgartner, P. Kocbek, The impact of relative humidity during electrospinning on the morphology and mechanical properties of nanofibers, Int. J. Pharm. 456 (2013) 125-134. doi:10.1016/j.ijpharm.2013.07.078.

[72] H.S. Bae, A. Haider, K.M.K. Selim, D.Y. Kang, E.J. Kim, I.K. Kang, Fabrication of highly porous PMMA electrospun fibers and their application in the removal of phenol and iodine, J. Polym. Res. 20 (2013). doi:10.1007/s10965-013-0158-9.

[73] V. Cirillo, V. Guarino, L. Ambrosio, Design of bioactive electrospun scaffolds for bone tissue engineering., J. Appl. Biomater. Funct. Mater. 10 (2013) 223-228. 
doi:10.5301/JABFM.2012.10343.

[74] H. Yoshimoto, Y.M. Shin, H. Terai, J.P. Vacanti, A biodegradable nanofiber scaffold by electrospinning and its potential for bone tissue engineering, Biomaterials. 24 (2003) $2077-$ 2082. doi:10.1016/S0142-9612(02)00635-X.

[75] Q. Zhang, S. Lv, J. Lu, S. Jiang, L. Lin, Characterization of polycaprolactone/collagen fibrous scaffolds by electrospinning and their bioactivity, Int. J. Biol. Macromol. 76 (2015) 94-101. doi:10.1016/j.ijbiomac.2015.01.063.

[76] M.A. Alvarez Perez, V. Guarino, V. Cirillo, L. Ambrosio, In vitro mineralization and bone osteogenesis in poly(E-caprolactone)/ gelatin nanofibers, J. Biomed. Mater. Res. - Part A. 100 A (2012) 3008-3019. doi:10.1002/jbm.a.34233.

[77] W. Xu, D. Karst, W. Yang, Y. Yang, Novel zein-based electrospun fibers with the water stability and strength necessary for various applications, Polym. Int. 57 (2008) 1110-1117. doi:10.1002/pi.2450.

[78] I. Bonadies, F. Cimino, V. Guarino, In vitro degradation of zein nanofibres for propolis release in oral treatments, Mater. Res. Express. 6 (2019) 075407. doi:10.1088/20531591/ab178e.

[79] S. Ali, Z. Khatri, K.W. Oh, I.S. Kim, S.H. Kim, Zein/cellulose acetate hybrid nanofibers: Electrospinning and characterization, Macromol. Res. 22 (2014) 971-977. doi:10.1007/s13233-014-2136-4.

[80] F. Yang, Y. Miao, Y. Wang, L.-M. Zhang, X. Lin, Electrospun Zein/Gelatin ScaffoldEnhanced Cell Attachment and Growth of Human Periodontal Ligament Stem Cells, Materials (Basel). 10 (2017) 1168. doi:10.3390/ma10101168.

[81] D. Dippold, M. Tallawi, S. Tansaz, J.A. Roether, A.R. Boccaccini, Novel electrospun poly(glycerol sebacate)-zein fiber mats as candidate materials for cardiac tissue engineering, Eur. Polym. J. 75 (2016) 504-513. doi:10.1016/j.eurpolymj.2015.12.030.

[82] I. Rajzer, E. Menaszek, R. Kwiatkowski, J.A. Planell, O. Castano, Electrospun 
gelatin/poly( $\varepsilon$-caprolactone) fibrous scaffold modified with calcium phosphate for bone tissue engineering, Mater. Sci. Eng. C. 44 (2014) 183-190. doi:10.1016/j.msec.2014.08.017.

[83] B. Chuenjitkuntaworn, T. Osathanon, N. Nowwarote, P. Supaphol, P. Pavasant, The efficacy of polycaprolactone/hydroxyapatite scaffold in combination with mesenchymal stem cells for bone tissue engineering., J. Biomed. Mater. Res. A. 104 (2015) 264-71. doi:10.1002/jbm.a.35558.

[84] M.C. Phipps, W.C. Clem, J.M. Grunda, G.A. Clines, S.L. Bellis, Increasing the pore sizes of bone-mimetic electrospun scaffolds comprised of polycaprolactone, collagen I and hydroxyapatite to enhance cell infiltration, Biomaterials. 33 (2012) 524-534. doi:10.1016/j.biomaterials.2011.09.080.

[85] X. Wu, L. Miao, Y. Yao, W. Wu, Y. Liu, X. Chen, W. Sun, Electrospun fibrous scaffolds combined with nanoscale hydroxyapatite induce osteogenic differentiation of human periodontal ligament cells, Int. J. Nanomedicine. 9 (2014) 4135-4143. doi:10.2147/IJN.S65272.

[86] S. Behera, D. Naskar, S. Sapru, P. Bhattacharjee, T. Dey, A.K. Ghosh, M. Mandal, S.C. Kundu, Hydroxyapatite reinforced inherent RGD containing silk fibroin composite scaffolds: Promising platform for bone tissue engineering, Nanomedicine Nanotechnology, Biol. Med. (2017) 0-14. doi:10.1016/j.nano.2017.02.016.

[87] B. Niu, B. Li, Y. Gu, X. Shen, Y. Liu, In vitro evaluation of electrospun silk fibroin/nanohydroxyapatite/BMP-2 scaffolds for bone regeneration, J. Biomater. 5063 (2016) 1-14. doi:10.1080/09205063.2016.1262163.

[88] S. Adewuyi, I. Cruz-maya, E. Onome, Design of Nano-Chitosans for tissue engineering and molecular release, (n.d.) 1-36.

[89] D. Van Hong Thien, S.W. Hsiao, M.H. Ho, C.H. Li, J.L. Shih, Electrospun chitosan/hydroxyapatite nanofibers for bone tissue engineering, J. Mater. Sci. 48 (2013) 1640-1645. doi:10.1007/s10853-012-6921-1. 
[90] R. Liu, X. Xu, X. Zhuang, B. Cheng, Solution blowing of chitosan/PVA hydrogel nanofiber mats, Carbohydr. Polym. 101 (2014) 1116-1121. doi:10.1016/j.carbpol.2013.10.056.

[91] N. Mohan, M.S. Detamore, Biomimetic Nanofibers for Musculoskeletal Tissue Engineering, Elsevier Inc., 2015. doi:10.1016/B978-0-323-32889-0.00004-2.

[92] M. Borjigin, C. Eskridge, R. Niamat, B. Strouse, P. Bialk, E.B. Kmiec, Electrospun fiber membranes enable proliferation of genetically modified cells, Int. J. Nanomedicine. 8 (2013) 855-864. doi:10.2147/IJN.S40117.

[93] V. Cirillo, V. Guarino, M.A. Alvarez-Perez, M. Marrese, L. Ambrosio, Optimization of fully aligned bioactive electrospun fibers for ???in vitro??? nerve guidance, J. Mater. Sci. Mater. Med. 25 (2014) 2323-2332. doi:10.1007/s10856-014-5214-4.

[94] K.E. Swindle-Reilly, C.S. Paranjape, C. a. Miller, Electrospun poly(caprolactone)-elastin scaffolds for peripheral nerve regeneration, Prog. Biomater. 3 (2014) 1-8. doi:10.1007/s40204-014-0020-0.

[95] S. Ferraris, V. Guarino, A. Cochis, A. Varesano, I.C. Maya, Coating of Sub-Micrometric Keratin Fibers on Titanium Substrates : A Successful Strategy for Stimulating Adhesion and Alignment of Fibroblasts and Reducing Bacterial Contamination, (n.d.) 1-7.

[96] S. Ferraris, V. Guarino, A. Cochis, A. Varesano, I. Cruz-Maya, C. Vineis, L. Rimondini, S. Spriano, Aligned keratin submicrometric-fibers for fibroblasts guidance onto nanogrooved titanium surfaces for transmucosal implants, Mater. Lett. 229 (2018) 1-4. doi:10.1016/j.matlet.2018.06.103.

[97] E.J. Torres-Martinez, J.M. Cornejo Bravo, A. Serrano Medina, G.L. Pérez González, L.J. Villarreal Gómez, A Summary of Electrospun Nanofibers as Drug Delivery System: Drugs Loaded and Biopolymers Used as Matrices, Curr. Drug Deliv. 15 (2018) 1360-1374. doi:10.2174/1567201815666180723114326.

[98] Š S. Zupančič, Core-shell nanofibers as drug delivery systems, Acta Pharm. 69 (2019) 131153. doi:10.2478/acph-2019-0014. 
[99] Z.X. Meng, X.X. Xu, W. Zheng, H.M. Zhou, L. Li, Y.F. Zheng, X. Lou, Preparation and characterization of electrospun PLGA/gelatin nanofibers as a potential drug delivery system, Colloids Surfaces B Biointerfaces. 84 (2011) 97-102. doi:10.1016/j.colsurfb.2010.12.022.

[100] P. Tipduangta, P. Belton, L.Y. Wang, H. Tang, M. Eddleston, S. Qi, Electrospun Polymer Blend Nano fi bers for Tunable Drug Delivery: The Role of Transformative Phase Separation on Controlling the Release Rate, (2016). doi:10.1021/acs.molpharmaceut.5b00359.

[101] S. Chou, D. Carson, K.A. Woodrow, Current strategies for sustaining drug release from electrospun nanofibers, J. Control. Release. 220 (2015) 584-591. doi:10.1016/j.jconrel.2015.09.008.

[102] S. Zupanc, S. Sinha-ray, S. Sinha-ray, J. Kristl, A.L. Yarin, Controlled Release of Cipro fl oxacin from Core - Shell Nano fi bers with Monolithic or Blended Core, (n.d.). doi:10.1021/acs.molpharmaceut.6b00039.

[103] A.L. Yarin, Coaxial electrospinning and emulsion electrospinning of core-shell fibers, Polym. Adv. Technol. 22 (2011) 310-317. doi:10.1002/pat.1781.

[104] A.M. Moydeen, M.S. Ali Padusha, E.F. Aboelfetoh, S.S. Al-Deyab, M.H. El-Newehy, Fabrication of electrospun poly(vinyl alcohol)/dextran nanofibers via emulsion process as drug delivery system: Kinetics and in vitro release study, Int. J. Biol. Macromol. 116 (2018) 1250-1259. doi:https://doi.org/10.1016/j.ijbiomac.2018.05.130.

[105] V. Guarino, I. Cruz-Maya, R. Altobelli, W.K. Abdul Khodir, L. Ambrosio, M.A. Alvarez Pèrez, A.A. Flores, Electrospun polycaprolactone nanofibres decorated by drug loaded chitosan nano-reservoirs for antibacterial treatments, Nanotechnology. 28 (2017) 505103. doi:10.1088/1361-6528/aa9542.

[106] V. Guarino, R. Altobelli, T. Caputo, L. Ambrosio, S. Caserta, P. Calcagnile, C. Demitri, Mono- and Bi-Phasic Cellulose Acetate Micro-Vectors for Anti-Inflammatory Drug Delivery, Pharmaceutics. 11 (2019) 87. doi:10.3390/pharmaceutics11020087.

[107] R.G. Bai, K. Muthoosamy, S. Manickam, Nanomedicine in Theranostics, Elsevier Inc., 2015. 
doi:10.1016/B978-0-323-32889-0.00012-1.

[108] H.H. Song, X. Gong, G.R. Williams, J. Quan, H.L. Nie, L.M. Zhu, E.L. Nan, M. Shao, Selfassembled magnetic liposomes from electrospun fibers, Mater. Res. Bull. 53 (2014) 280289. doi:10.1016/j.materresbull.2014.02.026.

[109] M. Jin, D.-G. Yu, X. Wang, C.F.G.C. Geraldes, G.R. Williams, S.W.A. Bligh, Electrospun Contrast-Agent-Loaded Fibers for Colon-Targeted MRI, Adv. Healthc. Mater. 5 (2016) 977985. doi:10.1002/adhm.201500872.

[110] M. Jin, D.G. Yu, C.F.G.C. Geraldes, G.R. Williams, S.W.A. Bligh, Theranostic fibers for simultaneous imaging and drug delivery, Mol. Pharm. 13 (2016) 2457-2465. doi:10.1021/acs.molpharmaceut.6b00197.

[111] M. Rasekh, Z. Ahmad, R. Cross, J. Hernández-Gil, J.D.E.T. Wilton-Ely, P.W. Miller, Facile Preparation of Drug-Loaded Tristearin Encapsulated Superparamagnetic Iron Oxide Nanoparticles Using Coaxial Electrospray Processing, Mol. Pharm. 14 (2017) 2010-2023. doi:10.1021/acs.molpharmaceut.7b00109.

[112] C. Zhang, Z.C. Yao, Q. Ding, J.J. Choi, Z. Ahmad, M.W. Chang, J.S. Li, Tri-Needle Coaxial Electrospray Engineering of Magnetic Polymer Yolk-Shell Particles Possessing DualImaging Modality, Multiagent Compartments, and Trigger Release Potential, ACS Appl. Mater. Interfaces. 9 (2017) 21485-21495. doi:10.1021/acsami.7b05580.

[113] Y. Wu, L. Li, Y. Mao, L.J. Lee, Static micromixer-coaxial electrospray synthesis of theranostic lipoplexes, ACS Nano. 6 (2012) 2245-2252. doi:10.1021/nn204300s.

[114] W. Wang, K.W.K. Yeung, Bone grafts and biomaterials substitutes for bone defect repair: A review, Bioact. Mater. 2 (2017) 224-247. doi:10.1016/j.bioactmat.2017.05.007.

[115] C. Zhu, Q. Wu, F. Wang, X. Zhang, F. Chen, X. Liu, Q. Yang, L. Zhu, Animal Models Used for Testing Hydrogels in Cartilage Regeneration., Curr. Stem Cell Res. Ther. 13 (2018) 517525. doi:10.2174/1574888X13666180514123103.

[116] A. Weigand, R.E. Horch, A.M. Boos, J.P. Beier, A. Arkudas, The Arteriovenous Loop: 
Engineering of Axially Vascularized Tissue., Eur. Surg. Res. 59 (2018) 286-299. doi:10.1159/000492417.

[117] C.G. Pfeifer, M.B. Fisher, V. Saxena, M. Kim, E.A. Henning, D.A. Steinberg, G.R. Dodge, R.L. Mauck, Age-Dependent Subchondral Bone Remodeling and Cartilage Repair in a Minipig Defect Model., Tissue Eng. Part C. Methods. 23 (2017) 745-753. doi:10.1089/ten.TEC.2017.0109.

[118] J.P. Schmitz, J.O. Hollinger, The critical size defect as an experimental model for craniomandibulofacial nonunions., Clin. Orthop. Relat. Res. (1986) 299-308. http://pbidi.unam.mx:8080/login?url=http://search.ebscohost.com/login.aspx?direct=true\&db $=\mathrm{cmedm} \& \mathrm{AN}=3084153 \&$ lang $=$ es\&site $=$ eds-live.

[119] Y. Li, S.-K. Chen, L. Li, L. Qin, X.-L. Wang, Y.-X. Lai, Bone defect animal models for testing efficacy of bone substitute biomaterials, J. Orthop. Transl. VO - 3. (2015) 95. doi:10.1016/j.jot.2015.05.002.

[120] M. Bottagisio, C. Coman, A.B. Lovati, Animal models of orthopaedic infections. A review of rabbit models used to induce long bone bacterial infections., J. Med. Microbiol. 68 (2019) 506-537. doi:10.1099/jmm.0.000952.

[121] I. Tessaro, V.T. Nguyen, A. Di Giancamillo, M. Agnoletto, F. Verdoni, M. Domenicucci, R. Scurati, G.M. Peretti, L. Mangiavini, Animal models for cartilage repair., J. Biol. Regul. Homeost. Agents. 32 (2018) 105-116. http://pbidi.unam.mx:8080/login?url=http://search.ebscohost.com/login.aspx?direct=true\&db $=$ cmedm\&AN=30644290\&lang $=$ es\&site $=$ eds-live.

[122] A. da Silva Morais, J.M. Oliveira, R.L. Reis, Small Animal Models BT - Osteochondral Tissue Engineering: Challenges, Current Strategies, and Technological Advances, in: J.M. Oliveira, S. Pina, R.L. Reis, J. San Roman (Eds.), Springer International Publishing, Cham, 2018: pp. 423-439. doi:10.1007/978-3-319-76735-2_19.

[123] I.R. Dias, C.A. Viegas, P.P. Carvalho, Large Animal Models for Osteochondral 
Regeneration., Adv. Exp. Med. Biol. 1059 (2018) 441-501. doi:10.1007/978-3-319-76735220.

[124] P.S. Gomes, M.H. Fernandes, Rodent models in bone-related research: The relevance of calvarial defects in the assessment of bone regeneration strategies, Lab. Anim. 45 (2011) 1424. doi:10.1258/la.2010.010085.

[125] F. Batool, M. Strub, C. Petit, I. Bugueno, F. Bornert, F. Clauss, O. Huck, S. Kuchler-Bopp, N. Benkirane-Jessel, Periodontal Tissues, Maxillary Jaw Bone, and Tooth Regeneration Approaches: From Animal Models Analyses to Clinical Applications, Nanomaterials. 8 (2018) 337. doi:10.3390/nano8050337.

[126] J.A. McGovern, M. Griffin, D.W. Hutmacher, Animal models for bone tissue engineering and modelling disease, Dis. Model. Mech. VO - 11. (2018). doi:10.1242/dmm.033084.

[127] R.M. Samsonraj, A. Dudakovic, P. Zan, O. Pichurin, S.M. Cool, A.J. van Wijnen, A Versatile Protocol for Studying Calvarial Bone Defect Healing in a Mouse Model., Tissue Eng. Part C. Methods. 23 (2017) 686-693. doi:10.1089/ten.TEC.2017.0205.

[128] A. Vajgel, N. Mardas, B.C. Farias, A. Petrie, R. Cimões, N. Donos, A systematic review on the critical size defect model., Clin. Oral Implants Res. 25 (2014) 879-893. doi:10.1111/clr.12194.

[129] D. Li, K. Zhang, C. Shi, L. Liu, G. Yan, C. Liu, Y. Zhou, Y. Hu, H. Sun, B. Yang, Small molecules modified biomimetic gelatin/hydroxyapatite nanofibers constructing an ideal osteogenic microenvironment with significantly enhanced cranial bone formation, Int. J. Nanomedicine VO - Ume 13. (2018) 7167. http://pbidi.unam.mx:8080/login?url=http://search.ebscohost.com/login.aspx?direct=true \&db $=$ edsdoj\&AN=edsdoj.2252ff76f60340f3baa7f2bc9e8a6a3d\&lang=es\&site=eds-live.

[130] X. Zhang, C. Wang, M. Liao, L. Dai, Y. Tang, H. Zhang, P. Coates, F. Sefat, L. Zheng, J. Song, Z. Zheng, D. Zhao, M. Yang, W. Zhang, P. Ji, Aligned electrospun cellulose scaffolds coated with rhBMP-2 for both in vitro and in vivo bone tissue engineering, Carbohydr. 
Polym. 213 (2019) 27-38. doi:https://doi.org/10.1016/j.carbpol.2019.02.038.

[131] X. Han, X. Zhou, K. Qiu, W. Feng, H. Mo, M. Wang, J. Wang, C. He, Strontiumincorporated mineralized PLLA nanofibrous membranes for promoting bone defect repair, Colloids Surfaces B Biointerfaces. 179 (2019) 363-373.

doi:https://doi.org/10.1016/j.colsurfb.2019.04.011. 


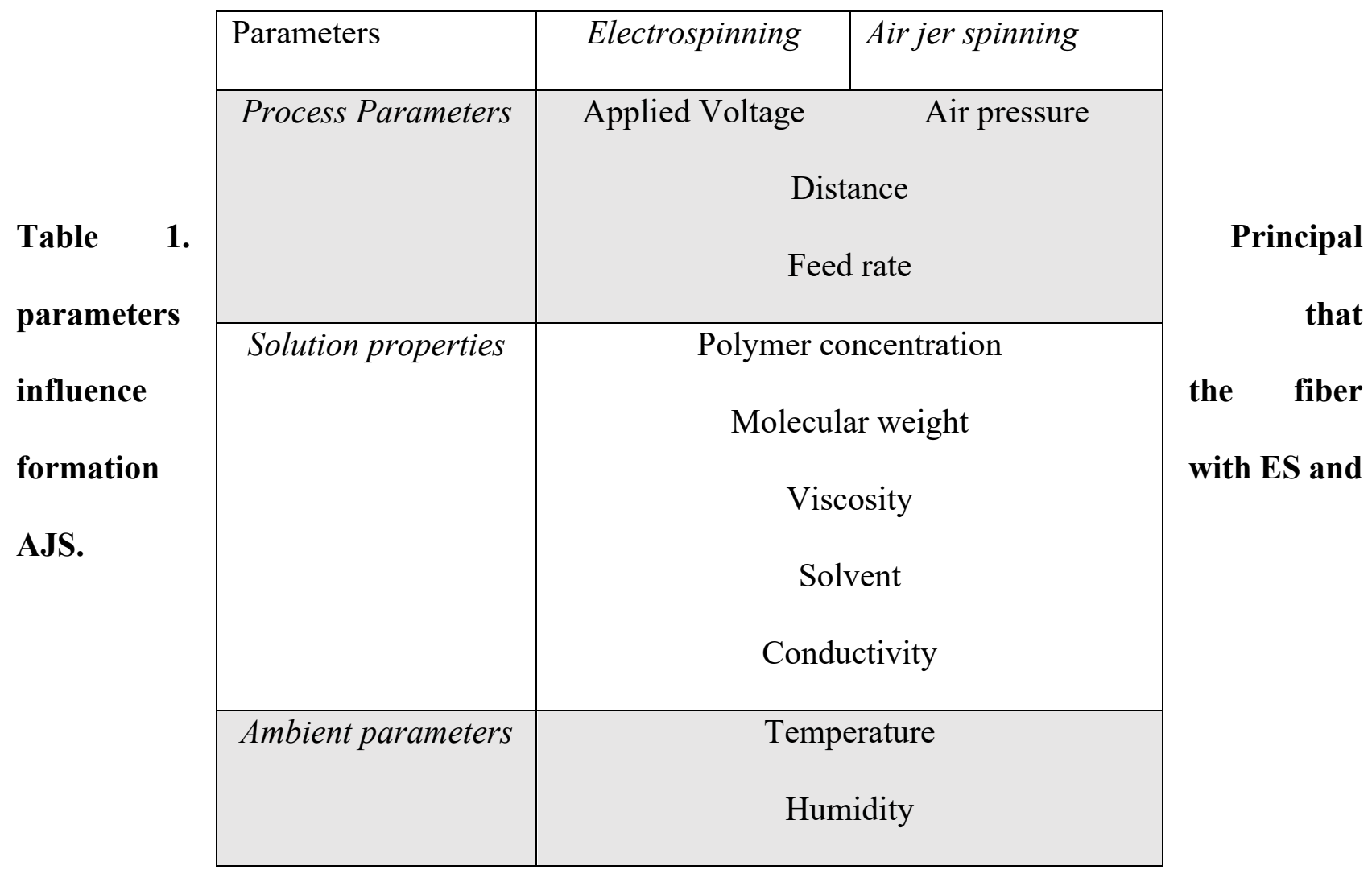




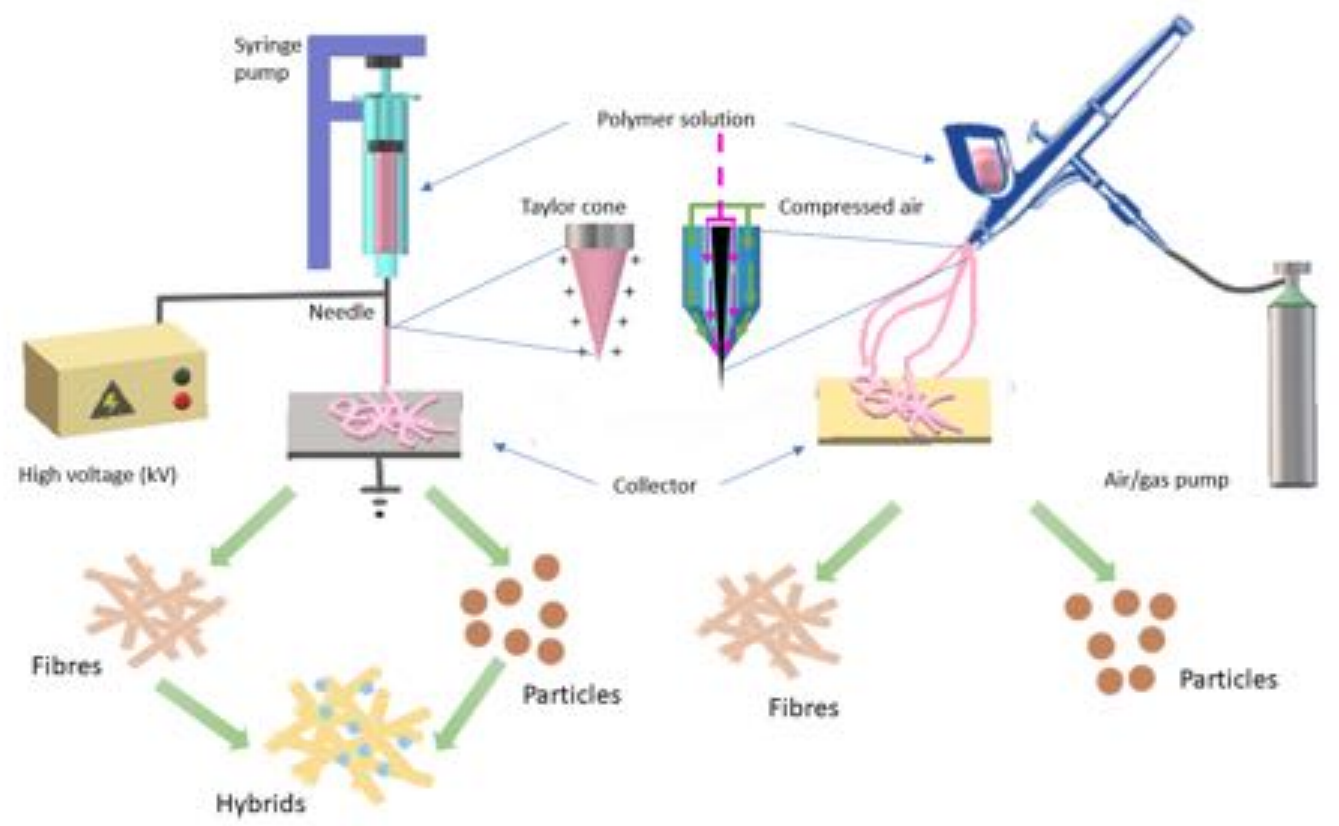

Figure 1: Scheme of the experimental setup of electric and non-electric assisted technologies. 


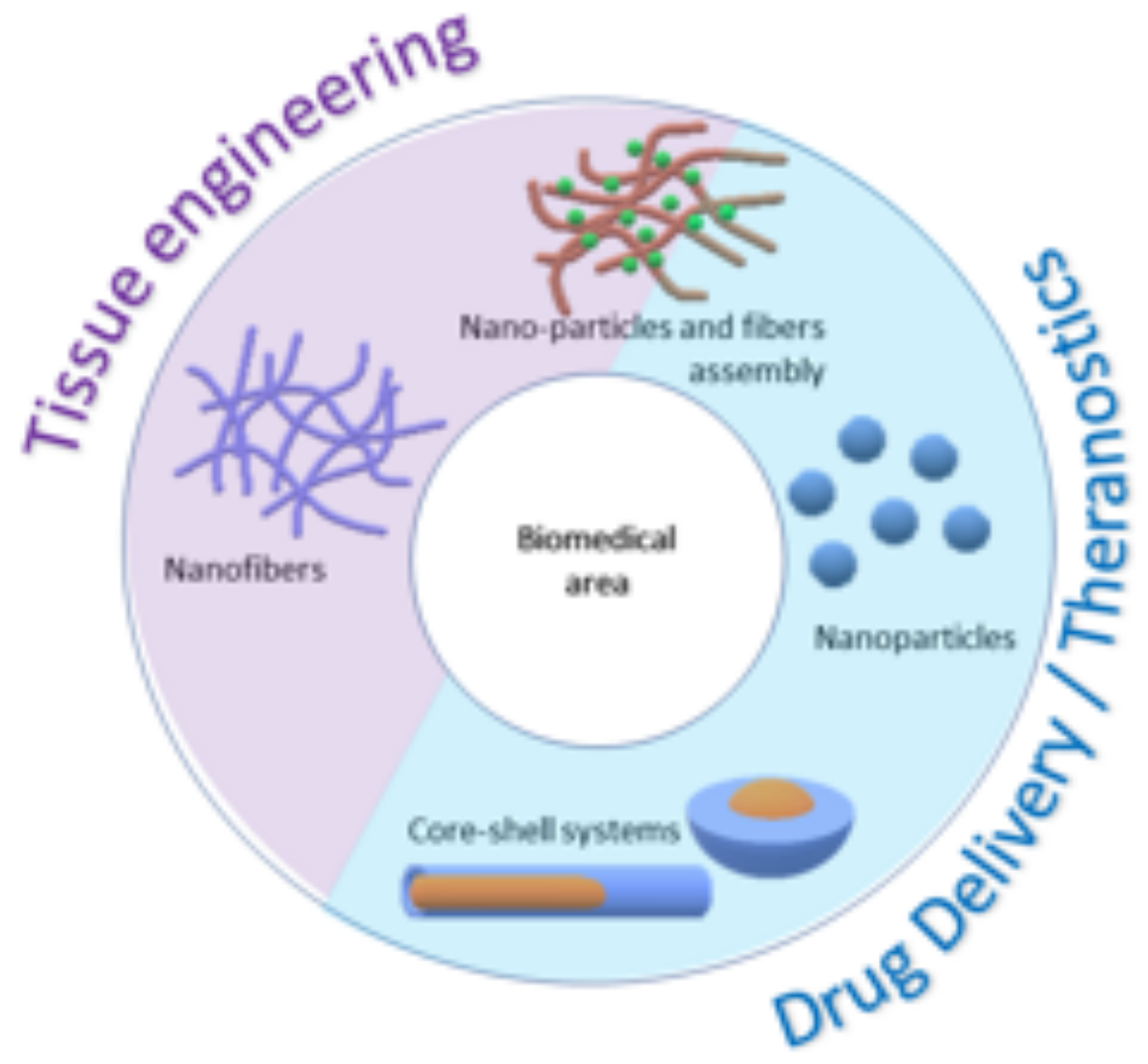

Figure 2: Application of nanostructures fabricated via electro and non electro spinning techniques in different biomedical area: Tissue engineering, Teranostics and drug delivery 


\title{
Nanocarbon for drug delivery Stefano Bellucci \\ INFN Laboratori Nazionali di Frascati, Via E. Fermi 40, 00044 Frascati, Italy
}

\begin{abstract}
In this paper we discuss the potential of carbon nanoparticles for the loading of drugs by hydrophobic interactions and $\pi$ - $\pi$ stacking, as well as bio-functionalization through covalent and non-covalent modifications. We review in vivo studies on the specificity of graphene and carbon nanotubes, which confirm their potential for the replacement and implementation of materials currently used for drug delivery.
\end{abstract}

\section{Introduction}

Each material has intrinsic properties and characteristics depending on its chemical and physical nature, its size, the type of chemical bond that composes it and its composition. This is valid from macromolecular systems to those of much smaller dimensions. Among all the various existing systems and compounds of interest are the nanostructured ones. Nanostructures are systems consisting of a set of atoms with dimensions in the order of the nanometer. Such nanostructures possess interesting properties that are generally neglected when dealing with macroscopic dimensions. The study of these characteristics has led to the discovery of important compounds.

Of interest has always been the chemistry linked to carbon, the element at the base of life. It is known that carbon is present in nature mainly in two allotropic forms, diamond and graphite. The first has a rigid structure, each atom is surrounded by four other atoms with a tetrahedral organization that makes it the hardest natural substance. At the same time, graphite has a planar organization: layers consisting of rings of six Csp2 atoms are superimposed in a single stable structure whose spatial organization depends on the arrangement of the planes. Between the planes, arranged perpendicularly, are the remaining non-hybrid p-orbitals that participate in an extended $\pi$ system with electronic density delocalized on the layers (Figure 2). The interactions between the layers are weak and are due to Van der Waals' forces, this allows them to flow with respect to each other. Furthermore, the unsaturated nature of the bonds in the planes allows the electrons to move through the planar ring structure, making graphite an electrical conductor. Graphite is the most thermodynamically stable allotropic carbon at room temperature.

Since 1985 the allotropic family of carbon has had a strong growth with the introduction of a class of compounds called buckminsterfullerenes, because of their shape reminiscent of the geodesic domes of the architect Buckminster Fuller. This opens the way for modern chemistry towards the realization of all the carbon nanostructures known today and research aimed at their technological application. Carbon-based nanometric structures, since the discovery of fullerene, have revolutionized chemistry from the point of view of the possibilities of synthesis and functionalization, but have also introduced many innovations in the field of nanotechnology for countless applications.

\section{Fullerenes}

Synthesized for the first time in 1985 by Kroto, Curl, Smalley and collaborators, fullerenes are spherical structures consisting of five and six atom carbon rings. The first fullerene identified was C60 (Figure 3): in this system all carbon atoms are equivalent, unlike the bonds between the rings. X-ray crystallography studies on C60 fullerene complexes have 
shown that the bonds between six-atom rings $(135.5 \mathrm{pm}$ ) have a more pronounced $\pi$ character than the bonds between six-atom rings and five-atom rings (146.7 pm). In fullerenes $\mathrm{C} 70$ the equivalence between carbon atoms is no longer valid and, in the structure, there are five types. By varying the number of carbon atoms in the structure of the fullerene, the spatial arrangement of the rings and therefore also the bonds vary.

Fullerenes have been studied to be used as molecular cages to encapsulate smaller molecules, it is also very interesting the research aimed at their functionalization. Hexagons and pentagons have different reactivity, a property to consider when studying the synthesis of fullerene compounds with externally bound groups. To date, fullerene synthesis methods are focused on a large scale and are based on the condensation of carbon in an inert atmosphere, vaporization by laser or other high-energy sources or controlled pyrolysis of aromatic hydrocarbons [1].

\section{Carbon nanotubes}

The carbon nanotube (CNT) is a cylindrical structure consisting of concentrically rolled graphene sheets. Their discovery is due to the Japanese Iijima who in 1991 observed nanometric filaments as a by-product of graphite vaporization for fullerene synthesis. Generally, nanotubes are multi-walled (MWCNT) with a diameter ranging from $2 \mathrm{~nm}$ inside to hundreds of nanometers for the outer tubes. However, there are also rarer, single-walled carbon nanotubes (SWCNT) with diameters ranging from $0.6 \mathrm{~nm}$ to $2 \mathrm{~nm}$. Depending on the folding mode of the graphene plane, different structures of the nanotube, Zigzag, Armchair and Chiral can be obtained.

Since their discovery, the methods developed to synthesize CNTs are manifold. An example is the Chemical Vapor Deposition (CVD) which is based on the thermal decomposition of gaseous hydrocarbons: subsequently the carbon atoms reorganize themselves as nanotubes on a suitable catalytic substrate [1]. This synthesis procedure is very simple and inexpensive but has a long time and requires the purification of the sample. A faster technique is the arc discharge: graphite electrode arcs are applied, and the anode is vaporized with subsequent deposition of the nanotubes. SWCNT and MWCNT can be obtained but their synthesis cannot be easily controlled and the amount of nanotubes obtained is limited compared to CVD. There are also more expensive techniques but with good SWCNT yields such as Laser Ablation. Although more difficult to obtain, SWCNTs are preferred because they are less flawed than MWCNTs.

The applications of nanotubes are varied and exploit their mechanical resistance, thermal and electrical conduction characteristics, the structural qualities that allow them to be used as nano-cavity and their chemical-physical properties such as hydrophobicity, the ability to form complexes given the extensive $\pi$ system on the side walls and their chemical stability. Countless researches have been addressed to the nanotube functionalization. In this sense there is to be considered the different reactivity of the regions: in fact, the outer surface has a lower stability in the half-fullerene terminal part with a higher quantity of five-term rings, while the side wall is less reactive and more difficult to functionalize. It should also be borne in mind that any covalent functionalization will modify the structure of the nanotube by creating deformations. In the same way, one can think of a non-covalent coordination with adequate functional groups by means of electrostatic interactions.

\section{Graphene}

Graphite consists of a set of multiple layers of carbon atoms with sp2 hybridization. A single plane isolated from the rest of the structure is called graphene. A single two-dimensional sheet of graphite was first synthesized in 2004 by Geim and collaborators. Graphene, with a thickness of about 340pm, is the basic structure of all other allotropic nanostructured carbon forms. Its properties have made it a material at the center of much research. In fact, graphene has a high resistance to fractures and deformations, a high thermal conductivity and has the conduction band connected to the valence band. It presents itself as a zero-gap planar semiconductor whose electrical properties can be modified by possible 
functionalization. The addition of functional groups can be performed in a covalent or non-covalent way, obviously in the first case the properties of the system will be more modified.

A very interesting compound of graphene is its oxidized form. Graphene oxide (GO) is water soluble, unlike graphene. Among the most important preparation procedures of $\mathrm{GO}$ is the synthesis of Hummer which uses acid oxidation with potassium permanganate $(\mathrm{KMnO} 4)$ and sulphuric acid $(\mathrm{H} 2 \mathrm{SO} 4)$. The active species that acts as an oxidizer is dimanganese heptoxide (Mn2O7) which selectively oxidizes unsaturated aliphatic and aromatic double bonds. Unfortunately, it is very complicated to predict the stoichiometry of graphene oxidation and consequently the structure of the final product.

The methods of graphene synthesis are divided into those that isolate it mechanically by exfoliation of graphite and those techniques that synthesize it from hydrocarbon precursors. The various existing methods can be evaluated on the basis of different requirements such as the purity of the obtained graphene (defined by the lack of intrinsic defects), the size of the flakes or layers obtained, the amount of graphene that can be obtained simultaneously, the difficulty of the chosen synthesis technique and the reproducibility of the method. Basically, there are two approaches to the preparation of graphene: a mechanical one in which the graphene is obtained from an already existing graphite crystal called exfoliation, the other chemical one in which there is a real synthesis [2].

The method used, the first time graphene was obtained by Novoselov and Geim in 2004, is mechanical exfoliation, using a simple adhesive tape [2]. The micromechanical exfoliation is an extremely simple technique, carried out by a repeated passage of the tape that allows to obtain graphene flakes composed of a few layers. Unfortunately, the size and thickness of the various flakes are very different and range from nanometers to several tens of micrometers. The amount of graphene that can be obtained through exfoliation is not very high, considering the lack of control of the method. On the other hand, the quality of the graphene is very high with almost no defects.

A completely different method involves the growth of graphene on a substrate. An advantage in taking this route is that the size of the graphene obtained does not depend on the size of the starting graphite crystal. The growth can start from carbon already present on the surface or depend on hydrocarbon precursors added during the process. In the first case we speak of epitaxial growth: graphene is prepared by heating and cooling a $\mathrm{SiC}$ crystal. Usually one or two layers of graphene are obtained on the Silicon face of the crystal, on the Carbon side more layers are produced. This technique depends very much on the synthesis conditions, such as temperature, heating and pressure. If temperature and pressure are too high, nanotube growth may occur. Metal catalysts such as Nickel are used, which lends itself very well to this role, because of its structure very similar to that of graphene.

The second case takes into account the growth from a gaseous hydrocarbon (such as methane or acetylene) which is the same mentioned for carbon nanotubes, the Chemical Vapor Deposition. The gaseous compounds decompose and recombine to form the graphene layer. There are several ways to achieve this, for example by heating the sample with a furnace, filament or plasma. Also in this case it is necessary to use a Nickel or Copper catalyst. A mixture of gases, e.g. $\mathrm{H} 2, \mathrm{CH} 4$ and $\mathrm{Ar}$ is heated to about 1000K: the decomposition of methane causes the diffusion of carbon in the metal catalyst. After cooling in Argon atmosphere, a graphene layer grows on the surface. In particular, the number of layers produced can be controlled according to the type of catalyst and by varying gas pressure ratios and synthesis temperature. Graphene has applications in various fields such as electronics where the mobility of its charges is exploited to make transistors and microchips faster than silicon ones, today the basis of all electronic devices. Used also in sensors, graphene, being a monoatomic material, can be exposed to the external environment on both sides of the sheet. An external modification (molecules, radiation, electrical charges) influences the charge transport of the graphene and this makes it 
an excellent material for the realization of sensors. This is followed by studies for the realization of graphene batteries or graphene sheets as molecular filters.

The application of graphene as a nano additive is interesting. Added to plastics or composite materials makes them more resistant and electrically conductive. There are already composite materials that use carbon or glass fibers for these purposes, but the use of graphene allows to obtain these results with minimal amounts of material. The high surface area of the nanostructure allows for maximum interaction with the surrounding material. The fascinating properties of graphene have prompted scientific and technological research to develop more and more techniques for its industrial production. Unfortunately, the preparation of individual graphene layers takes a long time and costs are not negligible. We are looking for cheaper and less time-consuming graphene structures that maintain the chemical, physical and chemical-physical characteristics of graphene.

A very interesting innovative material is Graphene nanoplatelets or GNP which consist of small systems of single superimposed graphene layers. GNP can be prepared in different ways. One technique is based on the use of graphite with intercalated chemical compounds. The intercalating chemicals are generally sulphates and nitrates which are specifically arranged between the sp2 carbon planes. When this material is subjected to thermal shocks of the order of thousands of degrees Kelvin, the interlayer substances vaporize causing the layers to move away and the formation of graphene nanoplates. The formation of sulphate and nitrate vapours creates a variation in the dielectric constant of the atmosphere and the formation of sparks: an electric arc has occurred. With simple techniques such as sonication or an ultrasonic bath, dispersed and not agglomerated GNP flakes are obtained. The lateral dimensions of the GNP obtained with this method range from 2 to $10 \mu \mathrm{m}$ and have a thickness between 2 and $9 \mathrm{~nm}$, which corresponds to a number of graphene layers ranging from 4 to about 11 [3-8].

Moreover, GNP are among the carbon nanostructures that are mostly used as fillers in composite materials to reinforce or add properties to the matrix. In fact, by dosing their quantity and calculating parameters such as percolation threshold, it is possible to obtain conductive materials that have excellent mechanical, thermal or resistance properties. Other countless applications are possible because of the properties very similar to graphene they possess, among these there is their use in electronic devices [9-18].

\section{Drug delivery systems}

The two-dimensional structure of graphene and the presence of delocalized $\pi$ electrons can be exploited for the loading of drugs through hydrophobic interactions and $\pi-\pi$ stacking. Furthermore, the availability of a large surface area (2600 $\mathrm{m}^{2} / \mathrm{g}$ ) allows for a high density of bio-functionalizations through covalent and non-covalent modifications. Several in vivo studies on the specificity of graphene have confirmed its potential for the replacement and implementation of materials currently used for bio-sensors and drug delivery [19]. Indeed, since its discovery graphene has shown excellent potential as a transport molecule (carrier) in drug delivery research. The high and defined surface area increases the opportunities for a targeted transfer from the administration site to the target site: polymer modifications and conjugation techniques lead, moreover, to an increase in biocompatibility. Many studies have been conducted on the transport of anticancer drugs, genes and peptides through graphene and related materials: the simple physisorption, for $\pi-\pi$, interactions, can be used to load several hydrophobic drugs that, through the following functionalization with antibodies, can lead to the selective destruction of cancer cells. Thanks to its small size, intrinsic optical properties, large surface area, low cost and non-covalent functional interaction with aromatic compounds, graphene has encouraging features for the nano-carrier approach. The extended molecular surface and interactions $\pi$ - $\pi$ or hydrophobic in particular, as can be seen in the references to the studies reported on the following page, contribute to the possibility of a high degree of loading of poorly soluble molecules, without compromising their potentiality or therapeutic efficiency. We also see how the use 
of graphene is extended to completely different fields, with extremely promising results in the biomedical field, with possible and future therapeutic application.

The Authors of [20] developed one of the first works in this field by synthesizing graphene oxide functionalized with polyethylene glycol (PEG) loaded with a camptothecin analogue (CPT), SN38. The NGO-PEG-SN38 complex exhibited good water solubility while maintaining the potentiality and efficiency of the loading. The complex also showed high cytotoxicity in HCT-116 cells, about a thousand times higher than the free drug: camptothecin is a cytotoxic quinolinic alkaloid that has the ability to inhibit the activity of the enzyme DNA-topoisomerase I. The CPT is it binds to the covalent I-DNA mouse complex with the formation of a highly stabilized ternary structure: this assembly leads to the nonrewinding of the DNA with consequent cellular apoptosis. The CPT, in particular, binds the enzyme and the DNA through the hydrogen bond: the most important part in the structure is the E-ring which interacts with three different H-bridges with the enzyme itself. The hydroxyl group at position 20 forms a hydrogen bond with the side chain of the enzyme at an aspartic acid residue (Asp533); the lactone is bound by two H-bridges to the amine group of Arg364. Camptothecin, in particular, is selectively cytotoxic for the cell in the S phase of DNA replication and its property is, in the first place, the result the conversion of a single-stranded fragment into a double-stranded fragment when the replication fork coincides with the breaking complex formed by DNA and CPT. In another study, the same group investigated the selective transport of Rituxan (a specific monoclonal antibody to the CD20 protein, found primarily on the surface of B cells of the immune system) conjugated with PEG-NGO. In both cases, non-covalent interactions $\pi-\pi$ they are exploited for drug loading on the surface of the PEG-NGO complex and for pH-dependent release of the same [21].

Joo et al. [22] reported studies of GO, loaded with Doxorubicin (DOX) again via interactions $\pi-\pi$, and how this shows a drug release in specific cell sites as a result of GSH triggering. Another research group reported as GO loaded with DOX, exhibiting a greater ability to release to an acidic $\mathrm{pH}(=5.3)$ due to the reduction of interactions between the drug and the carrier: it is in fact known that the $\mathrm{pH}$ of the cellular tumor environment is more acidic than healthy one, and this evidence has been exploited to obtain a targeted drug release at the target cell. The GO-DOX complex showed increased cell toxicity and promising tumor inhibition with a mortality range of $66 \%$ to $91 \%$. Other chemotherapeutic drugs, such as Paclitaxel and Methotrexate, loaded on GO for $\pi-\pi$ stacking and amide bonds, have shown surprising effects in the treatment of lung cancer and breast cancer, which resulted in an inhibition of tumor growth between $66-90 \%$ [23]. Graphene oxide, loaded with a second generation of photosensitizers, chlorine e6 (Ce6), has led to greater accumulation in tumor cells compared to previous treatments, allowing greater effectiveness in photodynamic therapy (PDT) [24].

Graphene-family nanomaterials (GFNs) have been conjugated with a series of bio-polymers such as gelatin and chitosan, acting as functionalizing agents for subsequent pharmacological application. Natural biopolymers are biocompatible, biodegradable and have low immunogenicity that can greatly reduce the toxic effect of graphene. Gelatin has been successfully used as a reducing and functionalizing agent for loading DOX onto graphene nanosheets (GS): the GelatinGS complex showed a greater loading capacity compared to the usual carriers due to the large surface area and the high interaction $\pi$. The tinnitus Gelatin-GS-DOX complex also exhibited high toxicity to MCF-7 cells for endocytosis. Chitosan, a linear cationic polysaccharide, obtained by alkaline deacetylation of chitin and composed of D-glucosamine and $\mathrm{N}$-acetyl-D-glucosamine bound by bonds $\beta$ (1-4), was used, in combination with graphene, for the loading of various compounds including ibuprofen, camptothecin and 5-fluoroacyl. Rana et al. [23] used GO functionalized with chitosan to transport ibuprofen (IBU), 5-fluoroacyl (5-FU) and CPT. The 5-FU showed a lower loading capacity due to the relatively hydrophilic character of the compound, to less interaction $\pi-\pi$ and in the presence of di-amide groups. In a subsequent study, Bao et al. [25] synthesized a chitosan-GO-CPT complex that showed characteristics of higher toxicity, compared to pure CPT, for HepG2 and HeLa cell lines. 
The conjugation of iron oxide nanoparticles with GFNs makes the latter superparamagnetic and can be useful in transport applications. Yang et al. [26] prepared a hybrid and superparamagnetic $\mathrm{GO}$ by addition of iron oxide nanoparticles $\left(\mathrm{Fe}_{3} \mathrm{O}_{4}\right)$ for precipitation methods followed by the loading of DOX. The magnetic hybrid showed a good aqueous dispersion before and after the loading with DOX with the formation of agglomerates in acid solution and subsequent redispersion in basic solution. This $\mathrm{pH}$-dependent release of $\mathrm{GO}-\mathrm{Fe}_{3} \mathrm{O}_{4}$ nanoparticles can be explored and optimized for the development of controllable release systems.

\section{Drug delivery: release controlled by endogenous stimuli}

The release of a molecule in an area of interest plays an important role in the field of drug delivery. Recently, drug delivery systems (DDS) graphene-based and responding to various endogenous stimuli such as $\mathrm{pH}$, redox potential and specific biomolecules, have been widely used to increase therapeutic efficacy and reduce unwanted effects of the drug used.

\section{Release mediated by $\mathrm{pH}$ variation}

DDS sensitive to extreme $\mathrm{pH}$ variations, such as those occurring in diseases such as ischemia, infections, inflammation and cancer, have been extensively studied in order to implement easily controllable systems. Since the tumor microenvironment is more acid when compared to healthy tissue, the search for $\mathrm{pH}$-dependent systems has been explored for effective use in cancer therapy. In acidic conditions, hydrophobic loads like Doxorubicin can be protonated, which reduces the amount of interactions $\pi-\pi$ and of the hydrophobic ones between the molecule under examination and the surface of the graphene, realizing a pH dependent system. In one of the first works in this sense [27] the graphene oxide was functionalized with polyethylene glycol (PEG) and studied as a two-dimensional nano-carrier for loading various substances. In this work, an antibody (anti-CD20, Rituxan) was conjugated with the PEG-GO system for a targeted and specific transport dependent on $\mathrm{pH}$ variation: starting from this study, various surface loading was used for the realization of a release model depends on the hydrogen ion concentration. For example, Pluronic F127 was used to make PF127-GO nanocomposites that exhibited a high loading capacity $(289 \% \mathrm{w} / \mathrm{w})$ and $\mathrm{pH}$-controlled release; similar characteristics have also been observed for lipid functionalizing lipid with DOX.

In order to increase the therapeutic efficacy and to reduce the side effects related to the administration of the drug, various systems based on graphene have been used: graphene sheets conjugated with a peptide (Chlorotoxin) (CTX-GO) have been prepared and used for the transport of DOX for non-covalent CTX-GO-DOX interactions. Chlorotoxin or CTX is a peptide of 36 amino acids that is found, together with other neurotoxins, in the venom of the yellow scorpion (Leiurus quinquestriatus), a scorpion of the Buthidae family. This toxin blocks the chlorine-dependent ion channels, acting as a neurotoxin: this fact, together with the fact that chlorotoxin exceeds the blood-brain barrier (BBB), and binds to the tumor cells of the gliomas, has suggested that the same can be usefully used in the treatment of the same tumor forms. The release of DOX proved to be $\mathrm{pH}$ dependent and showed good diffusion properties. In a subsequent study, Depan et al. [28] used folic acid conjugated with chitosan to modify nano-graphene oxide later used to transport DOX; in a recent work [29] nano-graphene oxide functionalized with dihydroartemisin (DHA) and transferrin was used in the development of a controlled-release chemotherapeutic drug: in this case a significant increase in tumor specificity was observed. In addition, hyaluronic acid (HA) was used for the modification of nano-graphene, aimed at the transport of an anti-tumor drug by means of endocytosis-mediated HA receptors.

Lastly, in the last few years, non-neutral nano-carriers, in which the surface charge can be modified from negative to positive by $\mathrm{pH}$ lowering inducing the loading or release of a drug, have received great interest in the field of DDSs. In a 
recent work [30], variable-load GO was developed: 2,3-dimethylmaleic (DA) and poly-allylamine (PAH) were used together to combine this reversible change to combine PEG- GO obtaining a nano-compound GO-PEG-DA. It has been studied how this ternary compound exhibits strongly stable negative charges under a physiological $\mathrm{pH}(\sim 7.0)$, but these fillers are rapidly converted into positive under weakly acidic conditions ( $\mathrm{pH}$ 6.8), at which the process of loading DOX onto GO-PEG-DA has been significantly increased. As a result, the GO-PEG-DA / DOX complex within the tumor microenvironment $(\mathrm{pH}>6.8$ ) showed greater efficacy in the destruction of drug-resistant MCF-7 / ADR cells, which are unlikely to be attacked in the presence of free DOX under the same $\mathrm{pH}$ conditions.

In summary, nano-graphene-based DDSs sensitive to $\mathrm{pH}$ changes were extremely promising for increasing the effectiveness of the usual cancer treatment drugs.

\section{- Redox stimulus-mediated release}

It is well known that the cellular redox environment is strictly controlled by the level of glutathione (GSH): GSH is a tripeptide with antioxidant properties, consisting of cysteine and glycine, bound by a normal peptide bond, and glutamate, which is instead linked to cysteine with an atypical peptide bond between the carboxylic group of the glutamate side chain and the cysteine aminic group (Fig. 1). Glutathione is a strong antioxidant, certainly one of the most important among those that the body is able to produce. Relevant is its action against both free radicals or molecules such as hydrogen peroxide, nitrites, nitrates, benzoates and others. The essential element for its correct functioning is the NADPH.

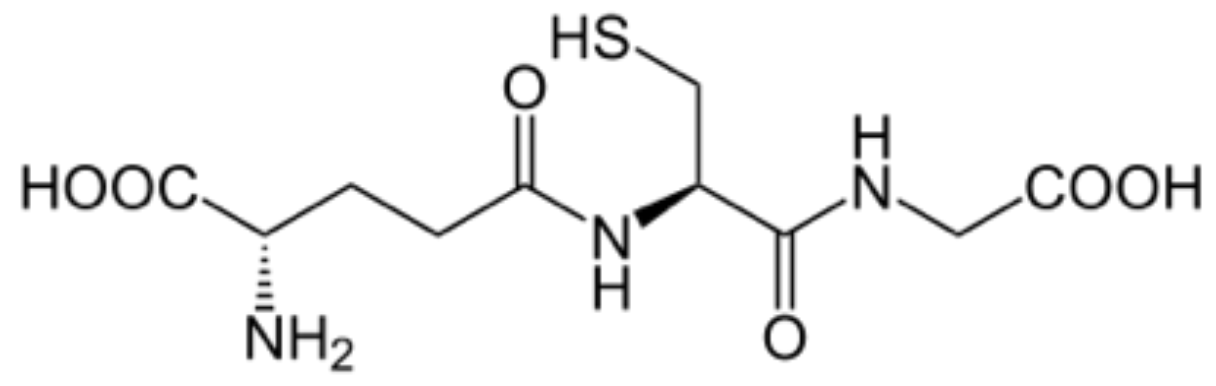

Figure 1 Structure of the glutathione tripeptide.

This molecule is a derivative of vitamin PP (nicotinic acid) with the function of oxidative-reductive cofactor of the enzyme glutathione reductase (or GSR). This enzyme regenerates reduced glutathione (GSH) from the oxidized molecule (or GSSG) through the electrons transferred from NADPH to GSSG. A decrease in GSH levels always leads to a consequent increase in the possibility of oxidative stress, while an excess of GSH in the cytoplasm increases the antioxidant capacity: the presence of glutathione could be exploited as a stimulus for the release of substances from drug delivery systems. In a paper by Shi et al. [31] a coating of PEG was used for the modification of nano-GO (NGO) by formation of disulfide bridges, leading to the formation of an NGO-SS-mPEG complex. This innovative system has been used for the transport of DOX by interaction $\pi$ - $\pi$ and showed the ability to be introduced into the cellular environment by endocytosis: in the presence of the cytoplasmic GSH concentration, the disulfide bridge of the NGO-SS-mPEG complex is rapidly reduced leading to the release of the loaded drug. In another work [32], NGO-Ag nanocomposites were prepared for intracellular drug delivery monitored by Raman scattering (SERS) and fluorescence spectroscopy. Doxorubicin is directly bound to the NGO-Ag nanocomposite for formation of disulfide bridges, which can then be broken down by intracellular GSH leading to diffusion of the loading. In addition to the possibility of redox-mediated release from molecules following 
superficial changes, in a subsequent work it was established that the degradability characteristics of the GO can be regulated by the redox sensitivity of the superficial coating [33]: it has been discovered that graphene oxide without any surface coating, although proving to be toxic for macrophage activity, can be gradually degraded through oxidative inducing enzymes such as HRP (peroxidase horseradish); at the same time, GO coated with biocompatible macromolecules, such as PEG or bovine serum albumin (BSA), does not show evident cellular toxicity but is degraded with difficulty in the organism. Therefore, to obtain functionalized and biocompatible GO, which can undergo enzymatic degradation, the latter has been conjugated with PEG by reversible disulfide bridges, thus obtaining GO-SS-PEG with negligible toxicity and considerable degradability. It is thus seen that a surface coating responsive to redox reactions can not only be used for the synthesis of intelligent DDSs, but also to mark and influence the biodegradability characteristics of the graphene itself.

\section{- Release mediated by biomolecules}

In addition to the release from $\mathrm{pH}$-dependent DDSs and redox balances, transport systems have been studied and developed in which the release mechanism is linked to the specific presence of a specific biological molecule. In a recent work [34] adenosine-5' - triphosphate (ATP), the main energetic molecule of cellular metabolism, has been chosen as a target for the control of the release capacity by nano-carrier of GO. In this work, a hybrid nano-aggregate GO-DNA was prepared containing a single strand of DNA1, DNA2, the aptamer of ATP (the aptamers are nucleic acids having the property of binding to a molecule or a protein) and GO, the latter used as a nano-platform for loading the drug. It has been seen that the individual strands of DNA1 and DNA2 together with the aptamer of the ATP can cross-link with each other on the surface of the GO, effectively inhibiting the release of DOX from the nano-sheets. In the presence of ATP, however, the interaction between the latter and the aptamer can induce the dissociation of the GO-DNA aggregate, promoting the release of DOX from the nano-sheets.

\section{Drug delivery: release controlled by exogenous stimuli}

In addition to endogenous stimuli, there are a number of external physical impulses potentially useful for controlling DDSs such as light, magnetic fields and temperature. Differently from what was discussed for endogenous stimuli (which were present within the same cellular environment), DDSs that respond to this type of stress, can show or exercise amplified therapeutic functions only under specific signals applied to the cellular environment from outside.

\section{- Release mediated by electromagnetic radiation}

By photothermal therapy (PTT) we mean the heating, generated by appropriate nanoparticles, following irradiation by near-infrared radiation (NIR). To date, a wide variety of organic and inorganic compounds, including nano-graphene, have been investigated as effective photothermal agents for direct tumor cell ablation; on the other hand, unlike high temperature heating (e.g. $>50^{\circ} \mathrm{C}$ ), a mild warming, which elevates the temperature of the tumor to $43-45^{\circ} \mathrm{C}$ and does not induce certain cell death, it has been discovered to be useful to increase the loading capacity of drugs (absorbers in NIR) and their subsequent release, for a more effective cancer therapy. In a series of works by different authors, nano-graphene and its derivatives have been reported as effective nano-carriers for the transport of a number of aromatic molecules. A 2011 work [35] shows how a photosensitizer, chlorine 6 (Ce6), can be effectively loaded on the surface of nGO-PEG for 
interactions $\pi-\pi$ and hydrophobic interactions. These have also noted how a mild photothermal heating induced by a laser radiation of $808 \mathrm{~nm}$, can greatly increase the loading of Ce6 by nGO-PEG, without, inter alia, inducing evident cytotoxicity at the cellular level and also increasing the efficacy of photothermal therapy against the tumor itself. In a subsequent work [36] reduced nano-graphene functionalized with PEG was used for the transport of resveratrol (RV), forming NrGO-PEG / RV: under NIR irradiation for a limited period of time, the RV released by the complex grew significantly, contributing, consequently, to an increased apoptosis. Therefore, as nano-carriers with strong NIR absorption, the graphene and its derivatives have proved promising DDSs mediated by electromagnetic radiation: in particular, a mild heating generated by photothermal effect, can lead to a significant increase in the control of the concentration of absorbed molecules and subsequently released, thus leading to the reduction of side effects currently present in healthy tissues.

\section{- Release mediated by magnetic fields}

In the past few years various nanocomposites based on graphene with peculiar magnetic properties, have been used for the realization of controlled delivery drug delivery. Iron oxide nanoparticles (IONPs) decorated with GO (GO-IONP) were first used by Yang et al. [37] as nano-carriers for the release of DOX mediated by $\mathrm{pH}$ variations: it was then discovered that cancer cells, incubated with GO-IONP-PEG-DOX under a magnetic field, showed a high loading of DOX, while a small absorption had been highlighted for the same cell culture in the absence of the applied field, thus demonstrating the effectiveness of the field in the elimination of cells following induced absorption.

\section{- Release mediated by temperature variation}

In addition to responses due to light and magnetic field, temperature variations have shown to be useful for the controlled release of molecules of biological interest. Therapy refers to the use of heat as a therapeutic tool for the treatment of diseases, such as tumors. Generally, in cancer therapy, heat is applied with the aim of increasing the temperature of the tissue by only a few degrees, in order to exploit the increased sensitivity of tumors to ionizing radiation and some drugs. Treatment, where the temperature range is roughly between 41 and $47^{\circ} \mathrm{C}$, it is called hyperthermia. At these temperatures, greater sensitivity to heat of tumors was observed experimentally compared to healthy tissues: when higher temperatures are applied, higher than about $50{ }^{\circ} \mathrm{C}$, the treatment is called thermotherapy; this catalyzes the rapid destruction of the fabric. However, at these temperatures, there is no difference in the sensitivity to heat between healthy tissue and neoplastic tissue, for this reason, thermotherapy must be applied accurately and in the right position because, when the tissue is heated, it necrotizes. The poly (N-isopropylacrylamide) (PNIPAM), one of the most known thermosensitive polymers with an LCST (is the critical temperature below which the components of a mixture become fully soluble in all compositions, is generally pressure-dependent increasing directly proportionally to the pressure itself, in the case of polymeric solutions, the LCST depends on the degree of polymerization, on the size, and on the composition and architecture of the polymer) easily modifiable in water, has been completely used as a material responding to variations of temperature. PNIPAM can also be used to functionalize GO through click-chemistry, obtaining GO-PNIPAM nanocomposites, subsequently loaded with IBU or CPT, which show dependent temperature release profiles [38]. 


\section{Toxicity of graphene and related materials}

As already seen, the GFNs range in shape, size, surface area, number of layers, side dimensions, chemical surface, hardness, density of defects and purity; all these properties significantly influence the interactions of GFNs with biological systems. Generally, GFNs with limited dimensions, sharp edges and rough surfaces are introduced into cells more easily when compared with larger and more regular members. Within this family, the mono-layer graphene has the maximum surface area allowed as each atom lies on a plane, providing an extremely high loading and functionalization capacity. For biological molecules, the members of the more stratified GFNs result in a lower adsorption capacity: the lateral dimensions, which range in a range between $10 \mathrm{~nm}$ and $100 \mu \mathrm{m}$, influence cellular uptake modalities, renal disposal and other biological interactions. Finally, since graphene is possible for different synthesis modes, for example mechanical exfoliation or processing of graphite intercalation compounds, it is inevitable that GFNs contain impurities, such as chemical additives or interlayer residues, which may include nitrates, sulphates and peroxides.

\subsection{Toxicity in vitro on mammal's cells}

An initial screening of new in vitro toxicity materials generally uses several cell lines. Literature data suggest that exposure to GFNs may result in cytotoxicity and / or genotoxicity in mammalian cells.

\section{- Graphene}

A comparative study measuring mitochondrial toxicity and cell membrane integrity in neuronal cells has suggested that the biological activity of graphene and SWCNTs strongly depends on their shape [39]. Following a 24h exposure, the metabolic activity of PC12 cells decreases in a variable manner: graphene leads to high toxicity at low concentrations and low toxicity at high concentrations, even more than compared to SWCNTs. The highest concentration of graphene used in these studies $(100 \mu \mathrm{g} / \mathrm{mL}$ ) Significantly increases the release of LDH (a total LDH level higher than normal is found in diseases such as: myocardial infarction, pulmonary infarction, acute viral hepatitis, toxic hepatitis, shock condition, severe anemia, muscular dystrophy, diabetes, renal failure, cirrhosis hepatic, leukemia and neoplasms, decreased values are found in subjects exposed to ionizing radiation) and the generation of reactive oxygen species (ROS). In addition, caspase- 3 activation (there are two types of caspases: initiator caspases (caspase-2, -8, -9, -10) that cut off inactive forms of other caspases called effector (caspase-3) , -6, -7) activating them, the effector caspases in turn will cut precise protein substrates, giving rise to the apoptotic process) suggests a time-dependent increase in the apoptotic process at a concentration equal to or greater than $10 \mu \mathrm{g} / \mathrm{mL}$. Yuan et al. [40] have compared the potential cytotoxicity of graphene and SWCNTs on the HepG2 cell line: overall, a concentration of $1 \mu \mathrm{g} / \mathrm{mL}$ of both nanomaterials led to the different expression of 37 proteins involved in cell metabolism, redox regulation, cytoskeletal formation and cell growth. An interesting discovery has been that graphene and SWCNTs produce different pathways of expression of calcium-binding proteins, thus indicating a different mode of action. Finally, pristine graphene has been identified as responsible for increased ROS concentration and apoptotic processes of macrophages of RAW 264.7 cell line, important for the innate immunity system.

\section{- Graphene Oxide (GO)}


The GO is the member of the graphene family whose toxicity has been most investigated [41]. Although the first toxicity studies did not show cell loading or effects on the morphology, viability and integrity of the membrane in cells affected by adenocarcinoma are influenced by exposure to GO [42]. This is in fact able to induce oxidative stress at a concentration equal to or greater than $10 \mu \mathrm{g} / \mathrm{mL}$. Hu et al. [43] using the same cell line, reported a cytotoxicity directly proportional to the concentration of the product, which can be strongly reduced by incubation with $10 \%$ of fetal bovine serum, due to the great capacity of protein absorption by the GO. Subsequently, the toxicity, genotoxicity, and mechanism of action of the GO were studied in a variety of animal and plant cell lines, including normal and immortalized cells, immune cells, stem cells and blood flow components. In studies including immortalized cells, the toxicity of graphene oxide was studied with the HepG2 line [44]. In this case, a decrease in fluorescence intensity was observed starting from the concentration of 4 $\mu \mathrm{g} / \mathrm{mL}$, which indicates possible damage to the plasma membrane; the loss of structural integrity of the plasma membrane is associated with a strong interaction of the GO with the double phospholipid layer. The use of TEM and SEM (electronic tunneling and, respectively, scanning microscopes) has shown that the GO has the ability to penetrate through the membrane, leading to an alteration of the cell morphology and an increase in the number of cells subject to apoptosis [45]. Concerning the mechanism of interaction, the authors concluded that damage to the plasma membrane and oxidative stress play a crucial role in the cytotoxicity of the component. Yuan et al. subsequently evaluated the toxicity of GO and oxidized SWCNTs in HepG2 cells [46]. Similarly to their previous study, a concentration of $1 \mu \mathrm{g} / \mathrm{mL}$ oxidized GO and SWCNTs lead to an alteration of the expression of proteins involved in metabolic pathways, cytoskeletal formation and cell proliferation, with a much less pronounced action of the GO compared to that of SWCNTs. Furthermore, a lower reduction in proliferation rate, a slightly modified cell cycle and a high concentration of intracellular ROS were observed in cells treated with GO, suggesting that GO has lower toxicity in HepG2 cells. The induction of cytotoxicity, genotoxicity and oxidative stress was also studied in pulmonary fibroblasts [47]. The MTT assay indicated a significant decrease in cell viability and an increase in toxicity following prolonged treatment, as well as the possibility of apoptosis at concentrations of $100 \mu \mathrm{g} / \mathrm{mL}$; DNA damage has been identified for all tested concentrations including that of $1 \mu \mathrm{g} / \mathrm{mL}$. The MTT assay, where the acronym indicates the 3- (4,5-dimetiltiazol-2-yl) -2,5-diphenyltetrazolium bromide compound, is a standard colorimetric assay for the measurement of the activity of enzymes that reduce the MTT to formazan (Fig. 2), giving the substance a blue / violet color. This occurs predominantly in the mitochondria and the assay can be used to determine the cytotoxicity of drugs or other types of chemically active and potentially toxic substances. In fact, the mitochondrial enzyme succinate dehydrogenase, is active only in living cells, and its function consists in cutting the tetrazolium ring of MTT (yellow) with the formation, consequently, of formazan (a blue salt).

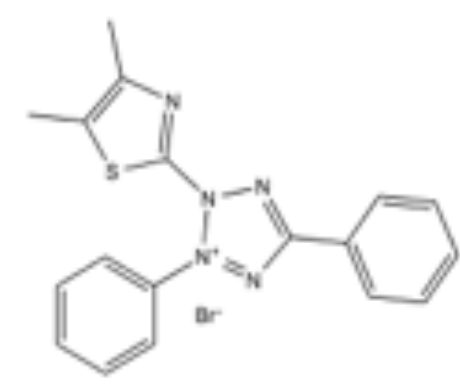

3-(4,5-dmethylthiszol-2-y 0$)-2,5-$ dighenyhetrazolum bromide

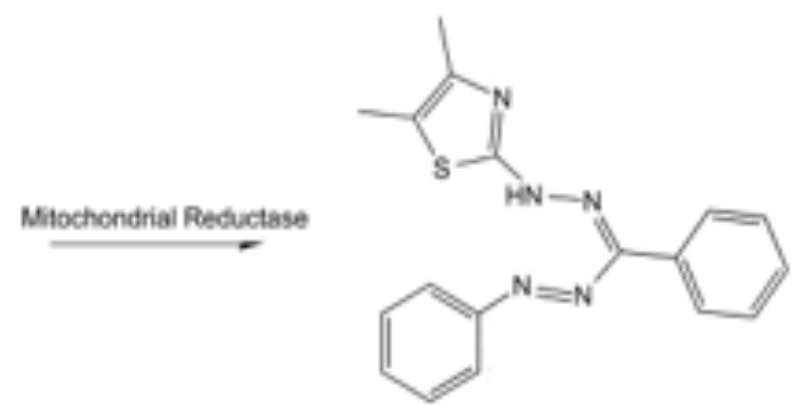

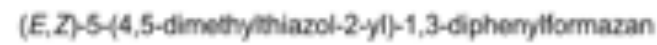

Figure 2 Reduction of MTT to formazan; working principle of the colorimetric assay. 


\section{- Reduced graphene Oxide (rGO)}

In the first studies of reduced graphene oxide toxicity on three different cell lines, it has been reported that the latter has less accentuated toxicity and therefore greater biocompatibility when compared with SWCNTs [48]. The diacetate fluorescine test showed significant cytotoxicity effects for rGOs with an average lateral size of $11 \mathrm{~nm}$, even at the lowest concentration of $1 \mu \mathrm{g} / \mathrm{mL}$ and following an hour of exposure [49]. The rGOs with an average lateral size of $3.8 \mu \mathrm{m}$ on the other hand, showed lower cytotoxicity compared to systems with dimensions of $91 \mathrm{~nm}$ and $418 \mathrm{~nm}$. Assays for the estimation of RNA flow from the cellular environment, indirect indicators of membrane damage, have confirmed a response strongly dependent on the size and shape of the RGO with hMSCs. The rGO of smaller size showed an outflow of RNA higher than that of a larger size; moreover, the rGO showed ROS levels 13-26 times higher than the control sample, thus suggesting the involvement of oxidative stress in the cytotoxic mechanism. In genotoxic studies, following an hour of rGO exposure with an average lateral size from $11 \mathrm{~nm}$ to $91 \mathrm{~nm}$, increases in the frequency of DNA damage and chromosomal aberrations at concentrations of $0.1 \mu \mathrm{g} / \mathrm{mL}$ and $1.0 \mu \mathrm{g} / \mathrm{mL}$. Using the MTT test, Hu et al. [43]. have found that nano-sheets of $\mathrm{rGO}$ with an average thickness of $4.6 \mu \mathrm{g}$, reduce cell viability from $47 \%$ to $15 \%$ at concentrations, respectively, of $20 \mu \mathrm{g} / \mathrm{mL}$ and $85 \mu \mathrm{g} / \mathrm{mL}$.

\section{- Functionalized graphene nanomaterials}

Many of the GFNs tend to aggregate into physiological solution due to electrostatic interactions and non-specific binding with proteins [50]. Thus, the development of functionalized GFNs led to increased solubility and biocompatibility, and consequently reduced cytotoxicity and genotoxicity. As said, two main methods are used for the synthesis of functionalized compounds: covalent interactions and non-covalent physisorption [50, 51]. Studies on covalent and noncovalent functionalization have shown a different decrease in toxicity and intensity of side effects in the members of GFNs.

In a study by Sasidharan et al., the pristine graphene toxicity was compared and functionalized in monkey renal epithelial cells, RAW 264.7 rat macrophages and primary components of the human blood stream [52, 53]. In monkey cells, the internalization of functionalized graphene within cells has not shown any short-term toxicity, while the accumulation of pristine graphene on the cell membrane leads to ROS-mediated apoptosis [52]. Finally, the treatment of mononuclear cells from peripheral blood with pristine graphene, produced a high expression of IL-8 and IL-6 (thanks to the secretion of interleukins, the cells of the immune system can regulate the activity of other cells, triggering one of the most important mechanisms of cellular communication at the level of the immune system, their action can be autocrine, paracrine and, in rare cases, endocrine) compared to treatment with functionalized graphene, indicating a smaller inflammatory capacity of the latter [53]. Unlike GO and rGO, which cause a strong aggregation response in the platelets, the aminofunctionalized graphene has no stimulating effects on human platelets; the intravenous administration of functionalized graphene does not lead to an increased lysis of erythrocytes or other diseases in mouse [54]. These results indicate how appropriately functionalized graphene can be potentially safe for in vivo biomedical applications. Functionalization, however, does not always lead to complete elimination of GFNs toxicity. 


\subsection{Toxicity in vivo on mammal's cells}

The possibility to use GFNs in DDS relies upon knowledge about their in vivo toxicity. Concerning GO, its toxicity was investigated by administration in guinea pigs [55]: no problems were found in mouse, exposed intravenously, at low GO concentrations $(0.1 \mathrm{mg})$ and medium ones $(0.25 \mathrm{mg})$. On the contrary, exposing the laboratory animals to a high dose $(0.4 \mathrm{mg})$ leads to a chronic toxicity. A substantial proportion of subjects died from suffocation within 1-7 days of administration due to blockage of the respiratory tract for the formation of agglomerates of GO. The maximum accumulation of GO occurs mainly in the lungs, followed by the liver and kidneys; the histopathological tissue examination indicates that the GO is basically eliminated by excretion into the bile, as only a small amount of material has concentrated in the kidneys. A similar study [56] has also shown that GO is rapidly subtracted from the bloodstream, then accumulated in the liver and lungs, with the larger oxide (1-5 $\mu \mathrm{m})$ concentrated in the airways and the thinner one $(110-500 \mathrm{~nm})$ retained in the liver. Also in this case, superficial changes significantly modulate the toxicity of graphene in vivo: a series of toxicological tests, performed using different routes of administration (intravenous, oral and intraperitoneal) for graphene and graphene functionalized with PEG were conducted on BALB / $\mathrm{c}$ mouse. One hour after the administration of $20 \mathrm{mg} / \mathrm{kg}$, nanosheets of PEG-graphene are distributed in a series of different organs; three days later, PEG-graphene is fundamentally concentrated in the reticuloendothelial system, including liver and kidney. Toxicological studies on nanosheets of PEG-graphene, have not reported cases of deaths or significant weight loss, over a period of 90 days after treatment. The biochemistry of the bloodstream and hematological analyzes have not identified any changes in the sensitive markers of liver and kidney including alanine aminotransferase, aspartate aminotransferase and alkaline phosphatase. In addition, no obvious systemic damage was found, except for discoloration in the liver and kidney, due to the accumulation of PEG-graphene in the first twenty days of treatment.

Recently, Yang et al. [57] investigated the biodistribution and potential toxicity of GO and a series of PEG-based derivatives with different sizes and surface coatings, following oral and intraperitoneal administration in BALB / $\mathrm{c}$ mouse of a dose of $4 \mathrm{mg} / \mathrm{kg}$. No marked loading at the tissue level was observed following oral administration, indicating a limited intestinal absorption of these nanomaterials; on the contrary, as a result of intraperitoneal treatment, the researchers observed a greater accumulation of PEG-GO derivatives, but not GO, in the reticuloendothelial system, including liver and kidney. Similar to other studies, histological examinations of dissected organs and haematological analyzes have revealed negligible changes in animals, although the nanomaterial persists within the organism for over three months. These results therefore suggest that the characteristics of in vivo toxicity depend to a considerable extent on the methods of administration.

A subsequent study investigated problems related to the inhalation of four carbon-based nanomaterials (MWCNTs, graphene, GNP, and carbon-black nano particulate matter) in adult Wistar rats [58]. The rats were exposed to atmospheres containing $0.1 \mathrm{mg} / \mathrm{m} 3,0.5 \mathrm{mg} / \mathrm{m} 3$ or $2.5 \mathrm{mg} / \mathrm{m} 3$ of MWCNT or $0.5 \mathrm{mg} / \mathrm{m} 3,2.5 \mathrm{mg} / \mathrm{m} 3$ or $10 \mathrm{mg} / \mathrm{m} 3$ of graphene, GNP and carbon-black for 6 hours / day for 5 consecutive days. No undesirable effects were observed following exposure of GNP or carbon-black, on the contrary, subjects exposed to a concentration of $2.5 \mathrm{mg} / \mathrm{m} 3$ of MWCNTs and graphene, had a higher number than the norm of lymphocytes, cytokines and an increased number activity of Y-glutamyltranspeptidase, LDH and alkaline phosphatase. Microgranulomas were also observed at the pulmonary level, with a more intense response provided by MWCNTs [58].

\section{Carbon nanotubes-based drug delivery systems}


We wish to conclude this review about the application of nanostructured carbon materials for the delivery of drugs, with a brief note about the use of carbon nanotubes in DDS, which has a longer history, with respect to that of graphene (see [59] for a comprehensive review about prospects and challenges in targeting nanodrugs for cancer therapy). It was recently shown that PEG modified carbon nanotubes armed with mAbs against the glucocorticoid-induced tumor necrosis factor receptor (GITR) were able to target with high selectivity an intra-tumor immune cell subset, i.e. specific "regulatory" $\mathrm{T}$ cells (Treg); suggesting that these nanodrugs can be used as scaffolds for efficient Treg-specific cancer immunotherapies [60-64]. In particular, we have shown that PEG-modified carbon nanotubes armed with anti-GITR mAbs (clone DTA-1) displayed an approximately 10-fold higher Treg versus effector T cells (Teff) targeting selectivity in the tumor tissue versus the spleen [60]. We speculated this phenomenon was due to the pathophysiological increase of Treg/Teff ratio in the tumor relative to the periphery and the (pathophysiological) increase in GITR density on intra-tumor versus peripheral Treg.

\section{Toxicity of carbon nanotubes materials}

A key challenge in nanotechnology is the more precise control of nanoparticle assembly for the engineering of particles with the desired physical and chemical properties. As we mentioned above, much research has been focusing on CNT as a promising material for the assembly of nanodevices, based upon new CNT-composite materials, in order to tailor their properties for specific applications. For instance, in [65], the tunable synthesis of multi-walled CNT-silica nanoparticle composite materials, was proposed. Instead of coupling prefabricated silica nanobeads to CNT, silica nanobeads were directly grown onto functionalized multi-walled CNT by reaction of tetraethyl- or tetramethyl-orthosilicate (TEOS or TMOS) with a functionalized CNT precursor, prepared by coupling aminopropyltriethoxysilane (APTEOS) to a functionalized multi-walled CNT through a carboxamide

bond, using a water-in-oil microemulsion to strictly control the nanobead size. Perhaps, the most valuable feature of this work was that the architecture of the obtained assemblies of covalently coated carbon nanotubes, with silica nanoparticles of different sizes, can be largely controlled by varying the conditions in the synthesis. Thus, the length of CNT is regulated by the oxidation time and the size of the nanobeads by using microemulsion conditions that yield micelles of a particular size. Indeed, Silica nanobeads were prepared in a water-in-oil microemulsion system in which the water droplets served as nanoreactors $[66,67]$. The size of the final nanospheres was mainly regulated by the dimension of the water droplets. Because the chemical properties of the silica surface are particularly versatile and silica can be doped with fluorescent [68], magnetic [69] or biological macromolecules [70], nanostructures with a wide range of morphologies suitable for different applications can be obtained, including providing an interface between living cells and biosensor arrays.

In [71] we synthesized and characterized three kinds of supramolecular nanostructures based on CNT and rutheniumcomplex luminophores. In the first nanostructure ruthenium-complex luminophores were directly grafted onto short oxidized single-walled carbon nanotubes. Hence, it consisted of short oxidized SWCNT covalently decorated by ruthenium-complexes that act as light-harvesting antennae by donating their excited-state electrons to the SWCNT. This nanocomposite represents an excellent donor-acceptor complex, which may be particularly useful for the construction of photovoltaic devices based on metallo-organic luminophores. In the second and the third nanostructures rutheniumcomplex luminophores were physically entrapped in silica nanobeads, which had been covalently linked to short oxidized single-walled carbon nanotubes or hydrophobically adsorbed onto full-length multi-walled carbon nanotubes. 
Since little was known at the time about the toxicity of CNTs, particularly of oxidized CNTs, we compared in [72] these two types of CNTs in a number of functional assays with human T lymphocytes, which would be among the first exposed cell types upon intravenous administration of CNTs in therapeutic and diagnostic nanodevices. We found that, especially for high concentration ( $>1 \mathrm{ng} /$ cell), carbon black is less toxic than pristine CNTs, therefore suggesting the relevance of the structure and topology (carbon black is amorphous) on the evaluation of the toxicity of a carbonaceous nanomaterial. Moreover, we found that oxidized CNTs are more toxic than pristine CNTs for both analyzed concentrations, although they are usually considered better suited for biological applications. This may well be because they are better dispersed in aqueous solution and therefore reach a higher concentration of free CNTs at similar weight per volume values.

For biotechnological uses [73], a high level of purity is required to avoid undesired toxic effects from impurities. Contaminants in SWCNT can be classified as carbonaceous (amorphous carbon and graphitic nanoparticles) and metallic (typically transition metal catalysts). It is well documented that nickel, which in combination with yttrium is used as a catalyst in the production of arc-discharged nanotubes, is cytotoxic [74]. Common SWCNT purification methods based on oxidation (nitric acid and/or air) have the potential disadvantage of modifying the CNT by introducing functional groups and defects. Other less rigorous purification techniques rely upon filtration, centrifugation and chromatography. Recently, electrophoresis of nitric acid-treated arc-discharged SWCNT was used to separate tubular carbon from fluorescent nanoparticles [75].

As we reported in [76], fluorescent nanoparticles were isolated from both pristine and nitric acid-oxidized commercially available carbon nanotubes that had been produced by an electric arc method. The pristine and oxidized carbon nanotubederived fluorescent nanoparticles exhibited a molecular-weight-dependent photoluminescence in the violet-blue and blue to yellowish-green ranges, respectively. The molecular weight dependency of the photoluminescence was strongly related to the specific supplier. We analyzed the composition and morphology of the fluorescent nanoparticles derived from pristine and oxidized nanotubes from one supplier. We found that the isolated fluorescent materials were mainly composed of calcium and zinc. Moreover, the pristine carbon nanotube-derived fluorescent nanoparticles were hydrophobic and had a narrow distribution of maximal lateral dimension. In contrast, the oxidized carbon nanotubederived fluorescent nanoparticles were superficially oxidized and/or coated by a thin carbon layer, had the ability to aggregate when dispersed in water, and exhibited a broader distribution of maximal lateral dimension. Thanks to these findings we have been able to design a new SWCNT purification method.

The functionalizing groups play a role which has been investigated in detail. In [77] we compared the in vitro cytotoxic, genotoxic and inflammatory effects of commercial pristine and $\mathrm{COOH}$-functionalized MWCNTs exposing human alveolar A549 and bronchial BEAS-2B epithelial cells to low concentrations of such CNTs with the attempt to investigate their toxic effects also in relation to functionalization and the cell susceptibility. It was possible to identify a suitable experimental model to study CNT toxicity on respiratory system. The present study showed for COOH-functionalized and pristine MWCNTs different effects on the two respiratory cells used. Bronchial cells are more responsive to cytogenotoxicity of functionalized MWCNTs and to inflammatory effects of pristine, and alveolar cells are more susceptible to cytogenotoxicity of pristine and to inflammatory effects of functionalized ones. In earlier works we studied the cytotoxicity and genotoxic/oxidative effects of pristine MWCNTs [78-83] and compared it with -OH functionalized MWCNTs [84]. Oxidative DNA damage was not observed for both CNTs. The results indicate a different cytotoxic mechanism, by membrane damage for MWCNTs and apoptosis for MWCNT-OH, that could be explained by a different cellular uptake. Moreover, we found an earlier genotoxic effect for MWCNT-OH. The findings suggest that further studies on functionalized CNTs are necessary before using them in several applications particularly in biomedical field. More recent toxicity assessments have dealt with self-assembled films made of CNT, such as the so-called buckypaper 
[85,86]. Lastly, for a comparative study of the cytotoxicity of pristine, as well as functionalized MWCNTs with hydroxyl (MWCNTs-OH) and carboxyl (MWCNTs-COOH) groups on the human cancer cell lines MCF-7, Caco-2, and HL-60 and normal human dermal fibroblasts (HFs), see [87].

\section{Acknowledgement}

The participation of A. Di Tinno and M.G. Fava to the early stages of this work is gratefully acknowledged.

\section{References}

[1] G. L. Miessler, P. J. Fischer, D. A. Tarr, Inorganic Chemistry, Pearson Ed., 2014

[2] N. Krane, "Preparation of graphene," Sel. Top. Phys. Phys. Nanoscale Carbon, pages $1-5,2011$.

[3] A. Dabrowska, S. Bellucci, A. Cataldo, F. Micciulla, A. Huczko, "Nanocomposites of epoxy resin with graphene nanoplates and exfoliated graphite: synthesis and electrical properties," Phys. Status Solidi B 251, 2599-602, 2014.

[4] A. Plyushch, J. Macutkevic, P. Kuzhir, J. Banys, Dz. Bychanok, Ph. Lambin,S. Bistarelli, A.

Cataldo, F. Micciulla, S. Bellucci, "Electromagnetic properties of graphene nanoplatelets/epoxycomposites," Composites Science and Technology 128, 75-83, 2016.

[5] A. Maffucci, F. Micciulla, A. Cataldo, G. Miano, S. Bellucci, "Bottom-up realization and electrical characterization of a graphene-based device," Nanotechnology 27, 095204, 2016.

[6] N.I. Volynets, D.S. Bychenok, A.G. Lyubimov, P.P. Kuzhir, S.A. Maksimenko, S.A. Baturkin, A.Y. Klochkov, M. Mastrucci, F. Micciulla, S. Bellucci, "Shielding properties of composite materials based on epoxy resin with graphene nanoplates in the microwave requency range," Tech. Phys. Lett. 42, 1141, 2016.

[7] M. Potenza, A. Cataldo, G. Bovesecchi, S. Corasaniti, P. Coppa, S. Bellucci, "Graphene nanoplatelets:thermal diffusivity and thermal conductivity by the flash method," $A I P A d v .7$ $075214,2017$.

[8] S. Bellucci, G. Bovesecchi, A. Cataldo, P. Coppa, S. Corasaniti, M. Potenza, "Transmittance and reflectance effects during thermal diffusivity measurements of GNP samples with the flash method," Materials 12, 696, 2019.

[9] L. Pierantoni, D. Mencarelli, M. Bozzi, R. Moro, S. Moscato, L. Perregrini, "Broadband microwave attenuator based on few layer graphene flakes," IEEE Transactions on Microwave Theory and Techniques 63 (8), 2491-2497, 2015.

[10] M. Bozzi, L. Pierantoni, S. Bellucci, " Applications of graphene at microwave frequencies," Radioengineering 24 (3), 661-669, 2015.

[11] A. Maffucci, F. Micciulla, A. Cataldo, G. Miano, and S. Bellucci, "Design, fabrication and electrical characterization of a low-cost and solvent-free graphene electronic device," 2015 IEEE International Conference on Microwaves, Communications, Antennas and Electronic Systems (COMCAS) 2015, 1-4. 
[12] A. Maffucci, F. Micciulla, A. Cataldo, G. Miano, S. Bellucci, "Bottom-up realization and electrical characterization of a graphene-based device," Nanotechnology, 27 (9), 095204, 2016.

[13] M. Yasir, P. Savi, S. Bistarelli, A. Cataldo, M.. Bozzi, L Perregrini, S. Bellucci, "A planar antenna with voltage-controlled frequency tuning based on few-layer graphene," IEEE Antennas and Wireless Propagation Letters 16, 2380-2383, 2017.

[14] M. Yasir, S. Bistarelli, A. Cataldo, M. Bozzi, L. Perregrini, S. Bellucci, " Enhanced tunable microstrip attenuator based on few layer graphene flakes," IEEE Microwave and Wireless

Components Letters 27 (4), 332-334, 2017.

[15] S. Bellucci, A. Maffucci, S. Maksimenko, F. Micciulla, M.D. Migliore, A. Paddubskaya, D. Pinchera, F. Schettino, " Electrical permittivity and conductivity of a graphene nanoplatelet contact in the microwave range," Materials 11, 2519, 2018.

[16] M. Yasir, S.. Bistarelli, A Cataldo, M. Bozzi, L. Perregrini, S. Bellucci, "Tunable phase shifter based on few-layer graphene flakes," IEEE Microwave and Wireless Components Letters 29 (1), 47-49, 2018.

[17] M. Yasir, S. Bistarelli, A. Cataldo, M. Bozzi, L. Perregrini, S. Bellucci, " Voltage-Controlled and Input-Matched Tunable Microstrip Attenuators Based on Few-Layer Graphene," IEEE Transactions on Microwave Theory and Techniques 68, 701 - 710, 2020.

[18] L. Ferrigno A. Cataldo, S. Sibilia, A. Maffucci, S Bellucci, "A monitorable and renewable pollution filter based on graphene nanoplatelets," Nanotechnology 31, 075701, 2020.

[19] S. Goenka, V. Sant, and S. Sant, "Graphene-based nanomaterials for drug delivery and tissue engineering," J. Controlled Release, vol. 173, pp. 75-88, 2014.

[20] Z. Liu, J.T.Robinson, X. Sun, H. Dai, "PEGylated nanographene oxide for delivery of waterinsoluble cancer drugs," J Am Chem Soc. 130(33), 10876-7, 2008.

[21] D.-J. Lim, M. Sim, L. Oh, K. Lim, H. Park, "Carbon-based drug delivery carriers for cancer therapy," Arch. Pharm. Res. 37. 43-52, 2014.

[22] Jae Yeon Joo, Gil Yong Park, Seong Soo A An, " Biocompatible and biodegradable fibrinogen microspheres for tumor-targeted doxorubicin delivery," Int J Nanomedicine. 10(Spec Iss), 101-111, 2015.

[23] V.K. Rana, M.-C. Choi, J.-Y. Kong, G.Y. Kim, M.J. Kim, S.-H. Kim, S. Mishra, R.P. Singh, C.-S. Ha, "Synthesis and drug-delivery behavior of chitosan-functionalized graphene oxide hybrid nanosheets," Macromol. Mater. Eng. 296(2), 131-140, 2010.

[24] Z.S. Singh, "Applications and toxicity of graphene family nanomaterials and their composites," Nanotechnol. Sci. Appl. 2016(9), 15-28, 2016.

[25] H. Bao, Y. Pan, Y. Ping, N.G. Sahoo, T. Wu, L. Li, J. Li, L.H. Gan, "Chitosan-functionalized graphene oxide as a nanocarrier for drug and gene delivery," Small 7(11), 1569-1578, 2011.

[26] K. Yang, L. Feng, X. Shi, Z. Liu, "Nano-graphene in biomedicine: theranostic applications," Chem. Soc. Rev. 42(2), 530-547, 2013.

[27] X. Sun, Z. Liu, K. Welsher,J.T. Robinson, A. Goodwin, S. Zaric, H. Dai, "Nano-graphene oxide for cellular imaging and drug delivery," Nano Res. 1 (3): 203-212, 2008.

[28] D. Depan, B. Girase, J.S. Shah, R.D. Misra, " Structure-process-property relationship of the polar graphene oxide-mediated cellular response and stimulated growth of osteoblasts on hybrid chitosan network structure nanocomposite scaffolds," Acta Biomater. 7(9),3432-45, 2011; Erratum in Acta Biomater. 8(3), 1395, 2012.

[29] L. Liu, Y. Wei, S. Zhai, Q. Chen, D. Xing, "Dihydroartemisinin and transferrin dual-dressed nano-graphene oxide for a pH-triggered chemotherapy," Biomaterials," 62, 35-46, 2015.

[30]. L. Feng, K. Li, X. Shi, M. Gao, J. Liu, Z. Liu, "Smart ph-responsive nanocarriers based on nano-graphene oxide for combined chemo- and photothermal therapy overcoming drug resistance," Adv. Healthc. Mater. 3(8), 1261-1271, 2014. 
[31] H. Y. Wen, H. Q. Dong, A. J. Shen, W. J. Xia, X. J. Cai, Y. Y. Song, X. Q. Li, Dr. Y. Y. Li, Prof. D. L. Shi, " Engineered Redox-Responsive PEG Detachment Mechanism in PEGylated NanoGraphene Oxide for Intracellular Drug Delivery, " Small 8, 760-9, 2012.

[32] H. Chen, Z. Wang, S. Zong, L. Wu, P. Chen, D. Zhu, C. Wang, S. Xu, Y. Cui, "SERSFluorescence Monitored Drug Release of a Redox-Responsive Nanocarrier Based on Graphene Oxide in Tumor Cells," ACS Appl. Mater. Interfaces 6, 20, 2014.

[33] G. P. Kotchey, B. L. Allen, H. Vedala, N. Yanamala, A. A. Kapralov, Y. Y. Tyurina, et al., "The enzymatic oxidation of graphene oxide, " ACS Nano 5, 2098-2108, 2011.

[34] R. Mo, T. Jiang, W. Sun, Z. Gu, " ATP-responsive DNA-graphene hybrid nanoaggregates for anticancer drug delivery ," Biomaterials 50, 67-74, 2015.

[35] B. Tian, C. Wang, S. Zhang, L. Feng, Z. Liu, " Photothermally Enhanced Photodynamic Therapy Delivered by Nano-Graphene Oxide," ACS Nano 5, 9, 7000-7009, 2011.

[36] J. Chen, H. Liu, C. Zhao, G. Qin, G. Xi, T. Li, X. Wang, T. Chen, "One-step reduction and PEGylation of graphene oxide forphotothermally controlled drug delivery," Biomaterials 35(18), 2014.

[37] X. Yang, X. Zhang, Y. Ma, Y. Huang, Y. Wang, Y. Chen, "Superparamagnetic graphene oxide-Fe3O4 nanoparticles hybrid for controlled targeted drug carriers," J. Mater. Chem.19(18), 2710-2714, 2009.

[38] K. Yang, L. Feng, and Z. Liu, "Stimuli responsive drug delivery systems based on nanographene for cancer therapy," Adv. Drug Deliv. Rev. 105, 228-241, 2016.

[39] X. Guo, N. Mei, "Assessment of the toxic potential of graphene family nanomaterials," J. Food Drug Anal. 22, 105-115, 2014.

[40] J. Yuan, H. Gao, C.B. Chin, "Comparative protein profile of human hepatoma HepG2 cells treated with graphene and single-walled carbon nanotubes: an iTRAQ-coupled 2D LC-MS/MS proteome analysis," Toxicol Lett. 207(3), 213-21, 2011.

[41] K. Yang, Y. Li, X. Tan, R. Peng, Z. Liu, " Behavior and toxicity of graphene and its functionalized derivatives in biological systems," Small 9(9-10), 1492-503, 2013.

[42] S.B. Liu, T.H. Zeng, M. Hofmann, E. Burcombe, J. Wei, R.R. Jiang, J. Kong, Y. Chen, "Antibacterial activity of graphite, graphite oxide, graphene oxide, and reduced graphene oxide: Membrane and oxidative stress," ACS Nano 5, 6971-6980, 2011.

[43] W. Hu, C. Peng, W. Luo, M. Lv, X. Li, D. Li, Q. Huang, C. Fan, " Graphene-based antibacterial paper," ACS Nano 4(7), 4317-23, 2010.

[44] C. Liao, Y. Li, S. C. Tjong, "Graphene Nanomaterials: Synthesis, Biocompatibility, and Cytotoxicity," Int J Mol Sci. 19(11), 3564, 2018.

[45] S.A. Loutfy, T.A Salaheldin, M.A. Ramadan,K.Y. Farroh, Z.F. Abdallah, T. Youssef, "Synthesis, Characterization and Cytotoxic Evaluation of Graphene Oxide Nanosheets: In Vitro Liver Cancer Model,"Asian Pac J Cancer Prev. 18(4). 955-961, 2017.

[46] J. Yuan, H. Gao, J. Sui, H. Duan, W.N. Chen, C.B. Chin, "Cytotoxicity Evaluation of Oxidized Single-Walled Carbon Nanotubes and Graphene Oxide on Human Hepatoma HepG2 cells: An iTRAQ-Coupled 2D LC-MS/MS Proteome Analysis," Toxicological Sciences 126(1), 149-61 2012. [47] A. Wang, K. Pu, B. Dong, Y. Liu, L. Zhang, Z. Zhang, W. Duan, Y.J. Zhu, " Role of surface charge and oxidative stress in cytotoxicity and genotoxicity of graphene oxide towards human lung fibroblast cells ," Appl Toxicol., 33(10):1156-64, 2013.

[48] S. Agarwal, X. Zhou, F. Ye, Q. He, G.C. Chen, J. Soo, F. Boey, H. Zhang, P. Chen, " Interfacing live cells with nanocarbon substrates," Langmuir 26(4), 2244-7, 2010.

[49] O. Akhavan, E. Ghaderi, A. Akhavan, "Size-dependent genotoxicity of graphene nanoplatelets in human stem cells," Biomaterials 33(32), 8017-25, 2012.

[50] Y. Pan, N.G. Sahoo, L. Li, "The application of graphene oxide in drug delivery," Expert Opin Drug Deliv. 9(11), 1365-76, 2012. 
[51] J. Liu, L. Cui, D. Losic, "Graphene and graphene oxide as new nanocarriers for drug delivery applications," Acta Biomater. 9(12), 9243-57, 2013.

[52] A. Sasidharan, L.S. Panchakarla, P. Chandran, D. Menon, S.V. Nair, C.N. Rao, M. Koyakutty "Differential nano-bio interactions and toxicity effects of pristine versus functionalized graphene," Nanoscale 3(6), 2461-4, 2011.

[53] A. Sasidharan, L.S. Panchakarla, A.R. Sadanandan, A. Ashokan, P. Chandran, C.M Girish, D. Menon, S.V. Nair, C.N. Rao, M. Koyakutty, "Hemocompatibility and macrophage response of pristine and functionalized graphene," Small 8(8), 1251-63, 2012.

[54] S.K. Singh, M.K. Singh, P.P. Kulkarni, V.K. Sonkar, J.J. Grácio, D. Dash, "Amine-modified graphene: thrombo-protective safer alternative to graphene oxide for biomedical applications," $A C S$ Nano 6(3), 2731-40, 2012.

[55] K. Wang, J. Ruan, H. Song, J. Zhang, Y. Wo, S. Guo, D. Cui, "Biocompatibility of Graphene Oxide," Nanoscale Res Lett. 6(1), 8, 2011.

[56] J.H. Liu, S.T. Yang, H. Wang, Y. Chang, A. Cao, Y. Liu, "Effect of size and dose on the biodistribution of graphene oxide in mice," Nanomedicine (Lond) 7(12), 1801-12, 2012.

[57] Yang K, Gong H, Shi X, Wan J, Zhang Y, Liu Z, "In vivo biodistribution and toxicology of functionalized nano-graphene oxide in mice after oral and intraperitoneal administration,"

Biomaterials 34(11), 2787-95, 2013.

[58] L. Ma-Hock, V. Strauss, S. Treumann, K. Küttler, W. Wohlleben, T. Hofmann, S. Gröters, K. Wiench, B. van Ravenzwaay, R. Landsiedel, "Comparative inhalation toxicity of multi-wall carbon nanotubes, graphene, graphite nanoplatelets and low surface carbon black," Part Fibre Toxicol. 10, 23, 2013.

[59] M. Bottini, C. Sacchetti, A. Pietroiusti, S. Bellucci, A. Magrini, N. Rosato, N. Bottini, "Targeted Nanodrugs for Cancer Therapy: Prospects and Challenges," Journal of Nanoscience and Nanotechnology 14, 98-114, 2014.

[60] C. Sacchetti, N. Rapini, A. Magrini, E. Cirelli, S. Bellucci, M. Mattei, N. Rosato, N. Bottini, and M. Bottini, "In Vivo Targeting of Intratumor Regulatory T Cells Using PEGModified SingleWalled Carbon Nanotubes," Bioconjug. Chem. 24, 852, 2013.

[61]. D. Mougiakakos, A. Choudhury, A. Lladser, R. Kiessling, and C. C. Johansson, "Regulatory T cells in cancer," Adv. Cancer Res. 107, 57, 2010.

[62]. T. L. Whiteside, "What are regulatory T cells (Treg) regulating in cancer and why?" Semin. Cancer Biol. 22, 327,2012.

[63]. M. P. Colombo and S. Piconese, "Regulatory-T-cell inhibition versus depletion: the right choice in cancer immunotherapy," Nat. Rev. Cancer. 7, 880, 2007.

[64]. J. Shimizu, S. Yamazaki, T. Takahashi, Y. Ishida, and S. Sakaguchi, "Stimulation of $\mathrm{CD} 25(+) \mathrm{CD} 4(+)$ regulatory T cells through GITR breaks immunological self-tolerance," Nat. Immunol. 3, 135, 2002.

[65] M. Bottini, L. Tautz, H. Huynh, E. Monosov, N. Bottini, M.I. Dawson, S. Bellucci, T. Mustelin, "Covalent decoration of multi-walled carbon nanotubes with silica nanoparticles," Chem. Commun. 6, 758, 2005.

[66] J. Esquena, Th.F. Tadros, K. Kostarelos, C. Solans, "Preparation of Narrow Size Distribution Silica Particles Using Microemulsions ," Langmuir 13, 6400. 1997.

[67] F.J. Arriagada, K. Osseo-Asare, "Synthesis of Nanosize Silica in a Nonionic Water-in-Oil Microemulsion: Effects of the Water/Surfactant Molar Ratio and Ammonia Concentration," $J$. Colloid Interface Sci. 211, 210, 1999.

[68] R.P. Bagwe, C. Yang, L.R. Hilliard, W. Tan, "Optimization of dye-doped silica nanoparticles prepared using a reverse microemulsion method," Langmuir 20, 8336, 2004.

[69] H.H. Yang, S.Q. Zhang, X.L. Chen, Z.X. Zhuang, J.G. Xu, X.R. Wang, "Magnetite-containing spherical silica nanoparticles for biocatalysis and bioseparations," Anal. Chem. 76, 1316, 2004.

[70] G. Fiandaca, E. Vitrano, A. Cupane, "Ferricytochrome c encapsulated in silica nanoparticles: structural stability and functional properties," Biopolymers 74, 55, 2004. 
[71] M. Bottini, A. Magrini, A. Di Venere, S. Bellucci, M.I. Dawson, N. Rosato, A. Bergamaschi, T. Mustelin,"Synthesis and characterization of supramolecular nanostructures of carbon nanotubes and ruthenium-complex Luminophores," J. Nanosci. Nanotechnol. 6, 1381, 2006.

[72] M. Bottini, S. Bruckner, K. Nika, N. Bottini, S. Bellucci, A. Magrini, A. Bergamaschi, T.

Mustelin,"Multi-walled carbon nanotubes induce T lymphocyte apoptosis," Toxicol Lett. 160, 121, 2006.

[73] R.H. Baughman, A.A. Zakhidov, W.A. de Heer., "Carbon nanotubes--the route toward applications ," Science 297, 787, 2002.

[74] M.D. Pulido, A.R. Parrish, "Metal-induced apoptosis: mechanisms," Mutat. Res. 533, 227, 2003.

[75] X. Xu, R. Ray, Y. Gu, H.J. Ploehn, L. Gearheart, K. Raker, W.A. Scrivens, "Electrophoretic analysis and purification of fluorescent single-walled carbon nanotube fragments," J. Am. Chem. Soc.126, 12736, 2004.

[76] M. Bottini, Ch. Balasubramanian, M.I Dawson, A. Bergamaschi, S. Bellucci, T. Mustelin, "Isolation andcharacterization of fluorescent nanoparticles from pristine and oxidized electric arcproduced single-walled carbon nanotubes," J Phys Chem B Condens Matter Mater Surf Interfaces Biophys. 110, 831, 2006.

[77] C.L. Ursini, D. Cavallo, A.M. Fresegna, A. Ciervo, R. Maiello, G. Buresti, S. Casciardi, S. Bellucci, S. Iavicoli, "Differences in Cytotoxic, Genotoxic, and Inflammatory Response of Bronchial and Alveolar Human Lung Epithelial Cells to Pristine and COOH-Functionalized Multiwalled Carbon Nanotubes," BioMed Research International Volume 2014, Article ID 359506, 14 pages.

[78] D. Cavallo, C. Fanizza, C.L. Ursini, S. Casciardi, E. Paba, A. Ciervo, A.M. Fresegna, R. Maiello, A.M. Marcelloni, G. Buresti, F.Tombolini, S. Bellucci, S. Iavicoli, "Multi-walled carbon nanotubes induce cytotoxicity and genotoxicity in human lung epithelial cells," Journal of Applied Toxicology 32 (6), 454-464, 2012.

[79] S. Bellucci, M. Chiaretti, A. Cucina, G.A. Carru, A.I. Chiaretti, "Multiwalled carbon nanotube buckypaper: toxicology and biological effects in vitro and in vivo," Nanomedicine 4 (5), 531-540, 2009.

[80] A. Di Sotto, M. Chiaretti, G.A. Carru, S. Bellucci, G. Mazzanti, "Multi-walled carbon nanotubes: lack of mutagenic activity in the bacterial reverse mutation assay," Toxicology Letters 184 (3), 192-197, 2009.

[81] M. Chiaretti, G. Mazzanti, S. Bosco, S. Bellucci, A. Cucina, F. Le Foche, G.A. Carru, S. Mastrangelo, A. Di Sotto, R. Masciangelo, A.M. Chiaretti, C. Balasubramanian, G. De Bellis, F. Micciulla, N.. Porta, .G Deriu, A Tiberia"Carbon nanotubes toxicology and effects on metabolism and immunological modification in vitro and in vivo," Journal of Physics: Condensed Matter 20 (47), 474203, 2008.

[82] M. De Nicola, S. Bellucci, E. Traversa, G. De Bellis, F. Micciulla, L. Ghibelli, "Carbon nanotubes on Jurkat cells: effects on cell viability and plasma membrane potential," Journal of Physics: Condensed Matter 20 (47), 474204, 2008.

[83] B.M. Rotoli, O. Bussolati, M.G. Bianchi, A. Barilli, C. Balasubramanian, S. Bellucci, E. Bergamaschi, "Non-functionalized multi-walled carbon nanotubes alter the paracellular permeability of human airway epithelial cells," Toxicology letters 178 (2), 95-102, 2008. [84] C.L. Ursini, D. Cavallo, A.M. Fresegna, A. Ciervo, R. Maiello, G. Buresti, S. Casciardi, F.Tombolini, S. Bellucci, S. Iavicoli, "Comparative cyto-genotoxicity assessment of functionalized and pristine multiwalled carbon nanotubes on human lung epithelial cells," Toxicology in Vitro 26, 831-840, 2012.

[85] S. Dinicola, M.G. Masiello, S. Proietti, P. Coluccia, G. Fabrizi, A. Palombo, F. Micciulla, S. Bistarelli, G. Ricci, A. Catizone, G. De Toma, M. Bizzarri, S. Bellucci, A. Cucina, "Multiwalled carbon nanotube buckypaper induces cell cycle arrest and apoptosis in human leukemia cell lines 
through modulation of AKT and MAPK signaling pathways," Toxicology in Vitro 29 (7), 1298$1308,2015$.

[86] O. Zeni, A. Sannino, S. Romeo, F. Micciulla, S. Bellucci, M.R. Scarfi, "Growth inhibition, cell-cycle alteration and apoptosis in stimulated human peripheral blood lymphocytes by multiwalled carbon nanotube buckypaper," Nanomedicine 10 (3), 351-360, 2015

[87] S. Bellucci, S. Dinicola, P. Coluccia, M. Bizzarri, A. Catizone, F. Micciulla, S. Bistarelli, G. Ricci, A. Cucina, "Biological Effects of Functionalized Multi-Walled Carbon Nanotubes on Human Cancer and Normal Cell Lines," J J Nanomed Nanotech. 1(1), 001, 2014. 


\title{
Bioactive Nanoceramics
}

Nupur Kohli ${ }^{1}$ and Elena García-Gareta ${ }^{2}$

1. Biomechanics Research Group, Department of Mechanical Engineering, Imperial College London, SW7 2AZ London, UK.

2. Regenerative Biomaterials Group, The RAFT Institute, Mount Vernon Hospital, HA6 2RN Northwood, UK.

\begin{abstract}
In the fields of regenerative medicine and tissue engineering, the shortcomings of autografts and allografts are driving the research of effective synthetic grafts. Particularly, nanotechnology applications are being extensively studied due to the nano-sized nature of the interactions between cells and the extracellular matrix of body tissues. This chapter provides an overview of the use of bioactive and bioresorbable nanoceramics for regenerative medicine purposes, focusing on tissue engineering strategies where release of the nanoceramics has a therapeutic effect for healing and regeneration. Especially, the strategy of incorporating nanoceramics in $3 \mathrm{D}$ polymeric matrices for bone, skin or peripheral nerve regeneration is reviewed and discussed.
\end{abstract}




\section{INTRODUCTION}

Nanoceramics are a type of nanoparticles comprised of ceramics, which are inorganic, heat-resistant and non-metallic solids that are composed of both metallic and non-metallic elements. On a macroscale, ceramics are brittle and rigid and break easily upon impact with hard objects. However, on a nanoscale, ceramics have been used extensively in a number of engineering applications as polishing agents, cutting tools, dielectrics and transducers, or sensors and catalyst agents as a few examples[1].

In the fields of regenerative medicine and tissue engineering, the shortcomings of autografts - limited availability, donor site morbidity and risk of infection at the donor site - and allografts - risk of immune rejection and disease transmission - have motivated the research of effective synthetic grafts that can substitute auto and allografts [2,3]. Particularly, nanotechnology applications have been extensively studied due to the nano-sized nature of the interactions between cells and the extracellular matrix (ECM) of tissues [4]. Some of these applications include scaffolds composed of nanofibers, nano-topographical modification of surfaces and materials, and the inclusion of nanoparticles, such as nanoceramics, into fibrous scaffolds, matrices and gels [4-7]. In the broader field of biomedicine, the potential for nanomedical devices, e.g. sensors for diagnosis and monitoring of diseases or high-surface drug-release agents, is enormous $[8,9]$.

Nanoceramics for biomedical applications, and more specifically tissue engineering/regenerative medicine and theranostics, are classified into bioactive, bioresorbable and bioinert.

Bioactive glass nanoceramics ( $\mathrm{nBG}$ ) present unique properties. As nBG degrade they release ions that can promote osteogenesis and angiogenesis. Moreover, these materials convert to a biologically active carbonated apatite material that firmly binds to bone [10]. Different types of bioactive glasses include silicate glasses such as $45 \mathrm{~S} 5$ or 13-93, which support in vitro proliferation and differentiation of osteoblast precursor cell lines and bone marrow stromal cells [11,12], or borate/borosilicate glasses such as 13-93B2, 13-93B3 or Pyrex ${ }^{\oplus}$. Interestingly, Yuan and colleagues reported osteoinductivity - the ability to induce local stem cells to differentiate into bone cells - of porous 45S5 bioactive glass [13]. The osteogenic properties of 45S5 bioactive glass could be a result of its dissolution products which stimulate osteoprogenitor cells at the genetic level [12]. Although bioactive glasses are widely known for enhancing bone repair and regeneration [14], they also have the capacity to stimulate skin repair, as the ionic products released from them in physiological conditions play critical roles in accelerating wound healing [15].

Bioresorbable nanoceramics are calcium-phosphate $(\mathrm{CaP})$ materials including, but not limited to, hydroxyapatite (HA), tricalcium phosphate, dicalcium phosphate dihydrate (brushite), octacalcium phosphate or biphasic calcium phosphate [16]. These different $\mathrm{CaP}$ compounds are biocompatible, bioactive, osteoconductive, and bioresorbable owing to their chemical similarity with the mineralised tissues found in the human body [3,16]. More importantly, these materials from a direct bond to bone tissue through formation of a bioactive apatite layer on their surfaces, which enhances their osteointegration [3,16]. Some of the CaP materials have even been found to be osteoinductive [3,16]. Solubilization and subsequent resorption of $\mathrm{CaP}$ materials depend on the solution's $\mathrm{pH}$, composition, and temperature as well as on the material's topography, particle size and pore size. Due to hydration, exposure of nano-CaP materials to biological fluids releases ions like $\mathrm{PO}^{3-}{ }_{4}, \mathrm{Ca}^{2+}$ and $\mathrm{HPO}^{2-}$, therefore regulating the functions of osteogenic cells [17]. However, CaP materials are brittle and their degradation rates are difficult to predict [3]. Thus, these materials are often used in combination with polymers, natural or synthetic, to form composites $[6,18,19]$.

Bioinert nanoceramics are titanium, alumina and zirconia-based materials. These materials are characterised by bioinertness, fracture toughness and high mechanical strength. For instance, titanium and its alloys possess corrosion resistance which makes them major players in reconstruction of bone tissue [17]. However, due to the bioinert nature of these materials, they will not be covered in this chapter.

The aim of this chapter is to provide an overview of the use of bioactive and bioresorbable nanoceramics for regenerative medicine purposes, focusing on tissue engineering strategies where release of the nanoceramics has a therapeutic effect for healing and regeneration.

\section{OVERVIEW OF BIOACTIVE NANOCERAMICS}


Bioceramics in general can be categorised as oxides or non-oxides. Alumina, zirconia and titania fall under the oxide category whereas silicon carbide and silicon nitride come under the non-oxide category (Figure 1). In terms of their chemical composition, they can be composed of phosphates, silicates or carbonates. Due to their physico-chemical properties, ceramics have been used as biomaterials for tissue engineering applications [8,20,21]. These ceramics are generally referred to as bioceramics due to their ability to exhibit biocompatibility. Examples of such ceramics include HA, silica-based glasses, alumina and zirconia, which have been used in orthopaedic applications [22]. These ceramics, whilst biocompatible, can be bioinert or bioactive. Bioinert substance does not interact with the physiological tissue where as a bioactive material, would react with the microenvironment and promote integration within the host tissue, consequently leading to bone formation. The two main bioactive ceramics that have gained wide interest in orthopaedics are HA and bioactive glasses [20,23].

Figure 1: Classification

$2.1 \quad$ Synthesis and
nanoceramics

A variety of techniques synthesis of their availability in are essentially divided bottom-up down approach involves breaking down of bulk structure, whereas a involves building up of building blocks to the simple terms, a topbreaking down of bulk bottom-up approach from atomic to nanosized material. There are various techniques used within both top-down and bottom-up approaches. For example, patterning, additive, subtractive and comminution techniques are common top-down approaches. Nanolithography, nanoimprint and nanoprinting fall under the patterning technique. Physical and chemical vapour deposition as well as atomic vapour deposition are examples of additive techniques. Dry and wet etching are examples of subtractive technique and grinding and milling come under the comminution techniques. Whilst top-down methods are cost-effective, bottom-up methods are preferred over top-down methods due to a more controlled fabrication resulting in a pure, homogenous structure [25]. Additionally, bottom-up approaches are more commonly applied for the synthesis of biological entities. However, bottom-up techniques tend to be cost-intensive. For bottom-up approaches, techniques such as colloidal synthesis, self-assembly and phase separation are used. A detailed approach on the synthesis of nanoparticles is provided in a recently published book chapter by Lei Yang [26]. For the purpose of this chapter, techniques and methods for producing bioactive nanoceramics only are explained.

An excellent study encompassing the various methods of calcium phosphate $(\mathrm{CaP})$ nanoceramic fabrication is listed in a paper by Hong et al. The authors explain in detail the advantages and disadvantages of various fabrication techniques for nanoceramic powders and coatings along with their biological behaviour [27]. Wet-chemical synthesis is one of the most commonly used bottom-up methods for the synthesis of ceramic nanoparticles including calcium phosphates [28], iron oxides, titanium oxides, etc [29]. In particular, the sol-gel method is widely used [30-32]. This method involves hydrolysis and polycondensation reactions. The main advantages of this method include lower processing temperatures, purity and the ability to synthesize multicomponent materials in various forms. It involves the preparation of a precursor mix which undergoes drying, chemical reactions, gelation and curing for conversion into a final product [33]. Different fabrication techniques result in different shapes and sizes of nanoceramics with different surface area. The CaP crystal size, shape and distribution as well as its deposition in the form of coating or application as powder will affect its properties and, 
thus, its potential applications [31,34]. Nanometer sized crystals of HA of roughly 5-20 nm in width are seen in the native bone tissue. Synthetic nano-HA (nHA) has been widely used in the orthopaedic industry in the form of powders, granules and porous blocks on its own or with polymeric composites. The properties of nHA such as surface grain size, porosity and wettability can be easily controlled to optimise its usage for clinical applications [31].

Another extensively studied and researched bioactive ceramic Bioglass ${ }^{\circledR}$ 45S5. It is a multicomponent oxide glass with four main components: $45 \% \mathrm{SiO}_{2}, 24.5 \% \mathrm{Na}_{2} \mathrm{O}, 24.4 \% \mathrm{CaO}$, and $6 \% \mathrm{P}_{2} \mathrm{O}_{5}$. Most bioactive glasses currently available are silicates and are based on these four constituents. Different from traditional silicate glasses, Bioglass ${ }^{\circledR}$ has low $\mathrm{SiO}_{2}$ content (less than $601 \%$ ), high $\mathrm{Na}_{2} \mathrm{O}$ and $\mathrm{CaO}$ contents and a high $\mathrm{CaO} / \mathrm{P}_{2} \mathrm{O}_{5}$ ratio. In addition to the silicate bioglasses, there also exists phosphate-based and borate-based glasses[33,35].

Bioactive glasses can be fabricated via two main methods including the conventional melt-quenching method or the solgel method (Figure 2). Both the techniques are comprehensively studied and reviewed [10,14,15,23,33,35,36].

\section{Figure}

2. Two main
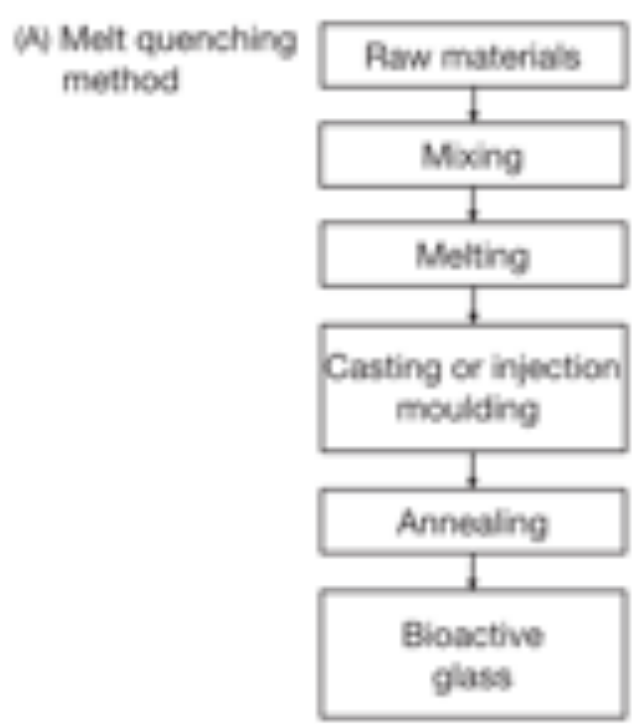

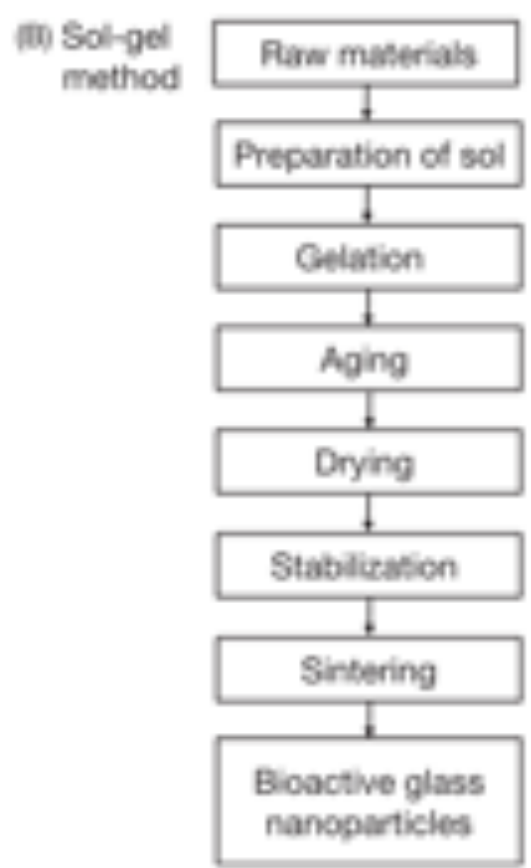

techniques for manufacturing bioactive glasses. (Reproduced with permission from Elsevier publishing group).

During melt quenching, certain quantities of raw materials such as $\mathrm{SiO}_{2}, \mathrm{Na}_{2} \mathrm{CO}_{3}, \mathrm{CaCO}_{3}$, and $\mathrm{Ca}_{2} \mathrm{P}_{2} \mathrm{O}_{7}$ are mixed initially, followed by melting at $1300-1450^{\circ} \mathrm{C}$ and annealing at $450-550^{\circ} \mathrm{C}$. For sol-gel synthesis of bioactive glasses, similar to $\mathrm{CaP}$ ceramics, the first step involves mixing the alkoxide or organometallic precursor together followed by the hydrolysis of these precursors. The hydrolysis process results in the formation of silanol groups that interact with each other to form silica network via polycondensation reactions. Following this, the process of gelation begins. With time, more and more particles interconnect forming a three-dimension network resulting a high viscosity liquid or otherwise referred to as a gel. Then, via a series of polycondensation and reprecipitation reactions, the gel is aged. The aged gel is then stabilized and sintered $[33,37]$.

The selection criteria for choosing the most suitable technique for manufacturing bioglass, depends on several factors since the overall aim is to manufacture a composition that would allow controlled bioactive behaviour for a successful clinical application. The melt-quenching method allows the melting and casting into molds shaped for specific applications. However, the technique maybe limited to the problem of presence of metallic ions forming unwanted alloys. On the other hand, the sol-gel method permits the expansion of the compositional range at lower processing temperatures without compromising the bioactivity of the system. The sol-gel method is most commonly used in the biomedical field due to the added benefit of functionalizing these systems by the addition of biomolecules during the preparation of sol. This is advantageous because the physico-chemical properties are not compromised as lower temperatures as used compared to melt-quenching. Moreover, these glasses can be doped with special ions to enhance biological functions such as antibacterial properties or angiogenesis $[10,33,35]$.

\subsection{Properties of nanoceramics}

Nanoceramics are nanometer-sized particles usually less than $100 \mathrm{~nm}$ in size. These nanoparticles have been reported to have the highest efficacy for cell and tissue integration due to a very high surface area-to-volume ratio, compared to submicron structures [38]. Nanoscale HA, for 
example, have enhanced functional properties compared to microscale HA due to their surface reactivity and homogenous ultrafine structure, which are imperative for graft integration following implantation. These nanoceramics have improved bioactivity due to an increased dissolution rate of the nanoscale structures which have a higher surface area exposed to the biological microenvironment compared to microscale structures. Additionally, nano-sized ceramics exhibit higher mechanical properties compared to micro-sized ceramics. However, for load bearing applications, the mechanical strength of HA ceramics is still too low. Therefore, HA ceramics are often used as coatings on metal implants to increase the implants biocompatibility and osteoconductivity [39]. A way to test bioactivity of developing biomaterials is to immerse them in simulated body fluid and examine the formation of HA layer on the surface of the materials after a certain time at $37^{\circ} \mathrm{C}$ [40]. The mineralization of bioactive glasses and $\mathrm{CaP}$ nanoceramics in SBF is simple and easier than the mineralization of their microstructure counterparts due to their intrinsic capacity to release bioactive ions. Apatite layer formation of micro-structured scaffolds often requires initial activation of the scaffold surface prior to immersion in SBF solution[41]. In this sense, bioactive nanoceramics can be classed as surface reactive biomaterials due to their ability to directly interact with biological microenvironment.

\begin{abstract}
Although nanoceramics have improved bioactivity and lead to a better tissue integration upon implantation, there exist several technical challenges in their production. High cost, poor reproducibility, ineffective control of variables, low yield of final products are common challenges during the synthesis of bioceramics[42]. Therefore, biphasic nanoceramics are being developed to over the challenges associated with single phase nanoceramics. Here, to overcome the poor degradation of $\mathrm{nHA}$, it is usually mixed with tri-calcium phosphate which has higher biodegradation capacity [41]. The properties of nanoceramics depend largely on the choice of synthesis method and the processing route. Therefore, it is imperative to choose the most suitable technique for preparing nanoceramics with desired properties and surface features. The main factors that determine the clinical success of a biomaterial, are its biocompatibility and functionality once its implanted in the body. Listed below are some examples of the clinically applicable nanoceramics and the current trends in the research of bioactive nanoceramics.
\end{abstract}

\title{
3. CURRENT BIOMEDICAL RESEARCH USING BIOACTIVE NANOCERAMICS
}

As mentioned in the introduction of this chapter, bioactive and bioresorbable nanoceramics have been shown to be osteoconductive and, some of them, osteoinductive. Therefore, these nanoparticles are highly attractive for bone repair and regeneration in orthopaedics or dentistry applications. However, while nanoceramics can be directly injected in small bone defects, they cannot be injected to repair large bone defects. This is because the apatitic structure obtained upon dissolution of the particles will not be porous enough to allow cell migration and proliferation and good vascularization of the new bony tissue [33]. Thus, macroporous structures are needed for optimal osteointegration. A popular and promising strategy to obtain such macroporous structures is to disperse bioactive nanoceramics in a polymeric matrix with an appropriate 3D shape. In this scenario, nanoparticles act as reinforcing agents of the polymeric matrix, thus increasing its mechanical properties while providing bioactivity and osteoconduction. The polymers used can be natural, like collagen, gelatin, chitin/chitosan, or alginates, or synthetic like poly (L-lactic acid), or poly (lactide-co-glycolide). The strategy of incorporating bioactive nanoceramics, particularly nBG, into a polymeric matrix can also be used for tissue engineering of soft tissues such as skin or peripheral nerve.

\subsection{Composite scaffolds of collagen or gelatin and nanoceramics for bone tissue engineering}

Collagen is the most frequently used protein in the fields of biomaterials and regenerative medicine due to its ubiquitousness in the human body[2,43]. In the case of mineralised body tissues like bone and dentin, collagen type I is the main component of their organic matrix. These tissues carry considerable compressive loads and the stiffness that they require could not be provided by the organic matrix alone. Therefore, in these tissues collagen is interfaced with plate-shaped mineral particles in the nano-meter scale made of a highly substituted hydroxyapatite (HA)[43]. A popular approach amongst biomaterial scientists and tissue engineers is to mimic the structure and composition of native body 
tissues. Following this approach for bone, combination of collagen or its hydrolysed version gelatin with ceramics is an obvious choice. Given the mineral in bone is nano-sized, combining collagen or gelatin with bioactive nanoceramics, particularly $\mathrm{nBG}$ and nanohydroxyapatite (nHA), is a very active area of research for the treatment of bone defects and fractures.

Since plastically compressed dense collagen gels mimic the structural and mechanical properties of native osteoid, Martelli and co-workers investigated the effect of hybridizing dense collagen gels with osteoinductive nBG particles as scaffolds for bone tissue engineering [44]. Immersion in simulated body fluid (SBF) for 3 days confirmed homogeneous growth of carbonated hydroxyapatite on the nanofibrillar collagen gel and by day 7, a 13-fold increase in the scaffold compressive modulus was observed. In vitro cell work with MC3T3-E1 pre-osteoblasts, showed the cells remained viable after 28 days in culture and accelerated osteogenic differentiation was observed in the absence of osteogenic supplements. Finally, no cell-induced contraction of the gels was seen. The authors concluded that the collagen/nBG gels were potentially suitable as osteoinductive cell delivery scaffolds for bone regeneration [44]. Hafezi and colleagues investigated a similar concept but using gelatin instead of collagen, and prepared gelatin/nBG scaffolds that guided bone formation in a rabbit ulnar critical-sized defect model and supported bone formation via intramembranous formation[45]. Also using gelatin and $\mathrm{nBG}$, Maji et al. fabricated gelatin/chitosan $/ \mathrm{nBG}$ scaffolds with $10 \%$ to $30 \% \mathrm{nBG}$ content using a sol-gel method followed by freeze-drying (Fig. 2) and chemical cross-linking with glutaraldehyde to improve their mechanical strength. The resulting scaffolds were $80 \%$ porous with a mean pore size range of $100-300 \mu \mathrm{m}$. The scaffolds containing the highest amount of $\mathrm{nBG}(30 \%)$ showed the maximum compression strength $(2.2 \pm 0.1 \mathrm{MPa})$. Furthermore, their cellular activity, in terms of attachment, proliferation and osteogenic differentiation, was improved compared to scaffolds without $\mathrm{nBG}$, thus demonstrating the potential beneficial effect of $\mathrm{nBG}$ for bone regeneration [46].

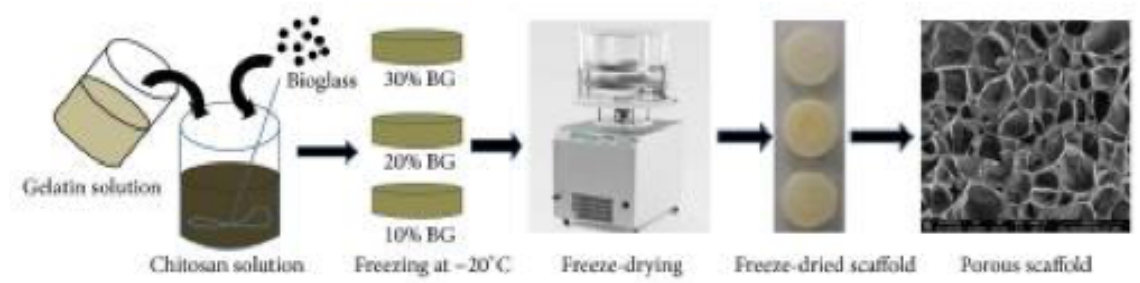

Figure 2. Fabrication of gelatin/chitosan/nBG scaffolds. Reproduced from Maji et al. 2016; Int J Biomater (Open Access article distributed under the terms of the Creative Commons Attribution License CC BY 4.0).

Bone repair and regeneration in avascular necrosis of the femoral head (ANFH) is difficult due to edema and high pressure caused by ischemia and hypoxia. Core decompression (CD) is commonly used for treating early ANFH, although its efficacy is still controversial. To improve the efficacy of CD on ANFH, Wang and colleagues proposed a tissue engineering strategy where bone marrow mesenchymal stem cells (BMSCs) were combined with a scaffold made of nHA/collagen I/poly-L-lactic acid (PLA) and implanted into the bone tunnel of CD [47]. 24 New Zealand rabbits with ANFH were randomly divided into three groups: Group A $(n=8), C D$; group B $(n=8), C D+n H A C /$ collagen/PLA; and group $\mathrm{C}(\mathrm{n}=8)$, CD+BMSCs-nHAC/collagen/PLA. Computerized tomography and histology revealed more capillaries and new osteoid tissue in group $\mathrm{C}$ in comparison with groups $\mathrm{B}$ and $\mathrm{A}$. Furthermore, new bone coverage rate and material degradation increased in group $\mathrm{C}$ compared with group $\mathrm{B}$. Thus, this study showed that the efficacy of $\mathrm{CD}$ could be improved with a tissue engineering strategy that combined stem cells, nHA, collagen and a synthetic polymer (PLA) [47]. The same amalgam of materials -nHA, collagen and PLA- was used by Liu and co-workers in combination with recombinant human bone morphogenetic protein 2 (rhBMP-2)-mediated dental pulp stem cells for reconstruction of alveolar bone defects [48].

The current clinical treatment of bone tumours requires surgery. Nevertheless, tumour cells may remain around bone defects after surgical intervention. Therefore, fabrication of scaffolds for both tumour therapy and subsequent bone regeneration is a clinical need. Rong and colleagues developed an osteoconductive and osteosarcoma-inhibitor porous scaffold made of collagen and nHA that was loaded with poly(lactic-co-glycolic acid) (PLGA) nanoparticles filled with adriamycin, a common chemotherapy medication[49]. The scaffold showed excellent extended-release properties and its extracts significantly inhibited the growth of osteosarcoma MG63 cells. In a femoral condyle defect rabbit model, no significant difference was seen between the adriamycin-loaded and unloaded scaffolds in terms of bone repair. In the immune response experiments after implantation into the rat muscle bag, the adriamycin-loaded scaffold showed remarkable biocompatibility. In an in vivo antitumor experiment, an improved antineoplastic effect and fewer adverse side effects were observed after implantation of the adriamycin-loaded scaffold in the tumor compared to direct intraperitoneal injection of adriamycin. Therefore, Rong and colleagues presented a potential solution for bone cancer treatment and subsequent bone repair[49]. 
Finally, an interesting study by Forero and co-workers presented the development of a scaffold made of gelatin, chitosan and nHA (Fig. 3), and some of them also incorporated nano-copper-zinc alloy. The suitable microstructure, the ability to introduce nanoparticles into the scaffold by a simple freeze-drying technique, and the scaffolds' biocompatibility indicated the potential of this new material for bone tissue engineering [50].

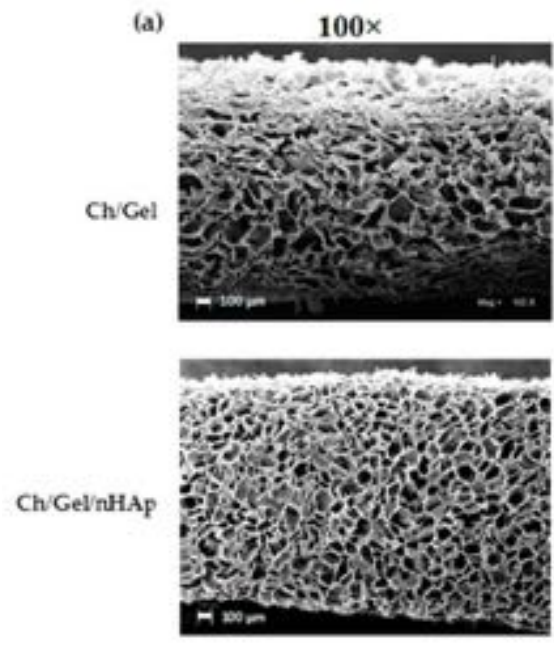

(b)

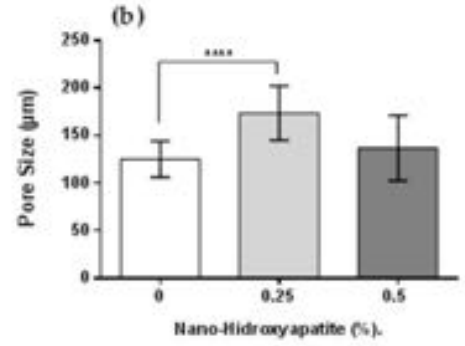

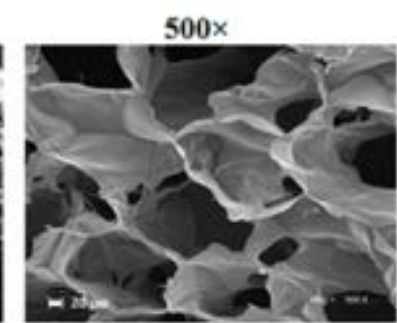

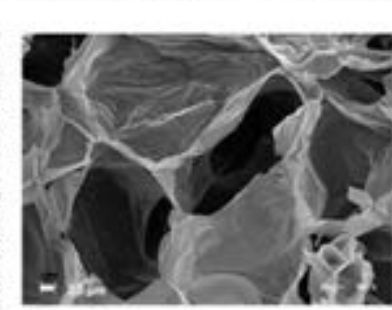

(c)

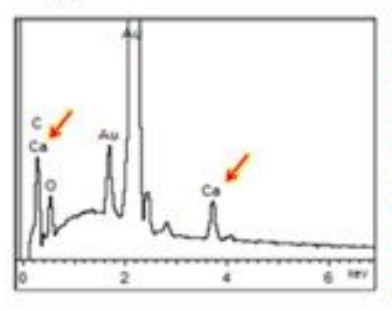

$5000 \times$

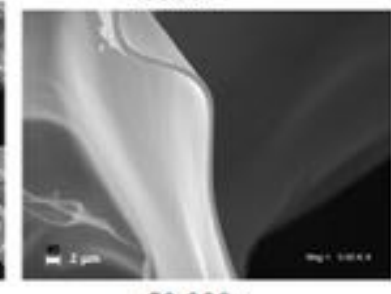

$50,000 x$

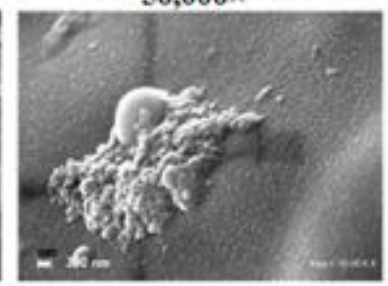

(d)

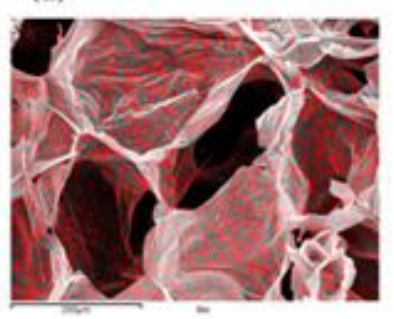

Figure 3. Scanning electron microscopy (SEM) images (a), pore size (b), and energy dispersive spectroscopy analysis (c) of gelatin/chitosan/nHA scaffold (Ch/Gel/nHAp). In (d) red points show the calcium distribution on the scaffolds' surface. **** $p<0.0001$ compared to control group. Reproduced from Forero et al. 2017; Materials (Open Access article distributed under the terms of the Creative Commons Attribution License CC BY 4.0).

\subsection{Composite scaffolds of chitin or chitosan and nanoceramics for bone tissue engineering}

Chitin and chitosan are natural polysaccharide-based polymers with attractive properties for their use in the engineering of various tissues (e.g. bone, skin, cartilage), wound healing and drug delivery. Chitin and chitosan are biocompatible, biodegradable, and possess antibacterial, non-antigenic and adsorption properties. Their main advantage is that they can be easily processed into various shapes and forms such as gels, micro and nanoparticles, nanofibers or beads. Scaffolds made of chitin or chitosan possess high, interconnected, gradient porosity. For bone tissue engineering purposes, it has been shown that chitin or chitosan-based scaffolds are osteoconductive and enhance bone formation in vitro and in vivo[18].

Chitin is obtained from the shells of marine crustaceans, sponges, insects or fungi and comprises 2-acetamido-2-deoxy$\beta$-D-glucose with a $\beta(1 \rightarrow 4)$ linkage. It is a white, hard, inelastic and hydrophobic polymer that is insoluble in water and most organic solvents. Partial deacetylation of chitin yields chitosan, which unlike chitin is soluble in dilute organic acids. Chitin and chitosan are structurally similar to glycosaminoglycans, a major component of the ECM of tissues. However, the main disadvantages of these natural polymers are their low mechanical properties and fast degradation. Therefore, their combination with nanoceramics to address both these issues is a popular strategy[18].

Incorporation of nHA into chitin or chitosan scaffolds is commonly achieved by homogeneously mixing precursor solutions for nHA with chitosan solution, which results in the co-precipitation of chitosan and nHA with an even distribution of the latter throughout the polymeric structure. Exogenous mineralisation of composite scaffolds prepared by this method has been shown when immersed in SBF. Generally, studies show that cellular attachment, viability, proliferation and osteogenic differentiation is enhanced on chitosan/chitin-nHA composites compared to chitosan/chitin only scaffolds [18]. An in vivo study in New Zealand white rabbits tibial defects by Lee and colleagues showed that total volume, bone volume, bone surface, trabecular thickness, trabecular number, and trabecular separation were higher in chitosan-nHA composite scaffolds in comparison with chitosan-mHA (microhydroxyapatite) scaffolds [51]. The nHA and mHA used to prepared the composite scaffolds had been isolated from marine fish bone and the scaffolds were 
prepared by the freeze-drying method [51]. Another in vivo study by Ma et al. investigated sponge-like chitosan-nHAp scaffolds cross-linked with glutaraldehyde in a standard critical-sized calvarial bone defects (6.5 mm) in Sprague-Dawley rats. The scaffolds were compared to control empty defects. After 1 week, histology showed a large number of cells anchored to the pores of the chitosan-nHAp scaffolds. After 2 weeks, new bone formation, both at the edge and in the centre of implants, was observed. After 5 weeks, significantly higher mineral content and volume of the new bone tissue was seen in the defects with implanted scaffolds compared to the control empty defects [52].

Combination of chitosan, nHA and other materials has also been explored [18,53-55]. For example, Lowe and co-workers prepared a composite scaffold of chitosan-nHA-fucoidan that showed high biocompatibility and excellent mineralization making them good candidates for bone tissue engineering [53].

Composites of chitin or chitosan incorporating $\mathrm{nBG}$ have also been widely researched and their preparation typically involves simple homogenization of the nanoparticles with a chitosan solution by blending or sonication [18,19]. The $\mathrm{nBG}$ particles are the composite's nanofiller and have a reinforcing effect as well as adding mineralization capability to the composite $[18,19]$. Sowmya and colleagues prepared scaffolds composed of $\beta$-chitin hydrogel and nBG for periodontal bone regeneration using a lyophilization technique. The authors showed that the composite scaffold had lower pore size than the control $\beta$-chitin scaffold as well as a slower degradation rate following immersion in PBS containing lysozyme for up to 28 days [56]. Peter and co-workers also observed a slower degradation rate for composite scaffolds made of chitosan and $1 \% \mathrm{nBG}$ that were prepared by blending the nanoparticles with a chitosan solution, chemical crosslinking with $0.25 \%$ glutaraldehyde and lyophilisation: the composite scaffolds showed a $5 \%$ weight loss after 1 week immersion in PBS containing lysozyme, compared to $25 \%$ weight loss observed for the chitosan only scaffolds [19]. Moreover, the composite scaffolds showed in vitro bioactivity when immersed in SBF for 7 days, and cytocompatibility when seeded with MG-63 cells [19].

\subsection{Alginate-based scaffolds incorporating nanoceramics for bone tissue engineering}

Alginates are natural polysaccharide-based linear anionic copolymers of (1-4)-linked $\beta$-mannuronic acid and $\alpha$-guluronic acid monomers [57]. They are primarily obtained from brown seaweed but can also be derived from bacteria. An important property of alginates is gelation and therefore, alginates are widely used as a gelling agent in the food industry, pharmaceuticals, and biomedicine in general. Alginate-based hydrogels display a physical structure that is similar to that of the native ECM of tissues. Furthermore, they possess gentle gelling kinetics, biodegradability, biocompatibility, and low toxicity [57]. Alginate-based hydrogels are being extensively researched as scaffolds for tissue engineering [58]. Alginate-based hydrogels possess mechanical integrity to produce scaffolds and can easily encapsulate cells during the hydrogel formation process. In addition, alginates are suitable as inks and bioinks, when incorporating cells, in various 3D printing techniques [57].

Alginate-based hydrogels can incorporate bioactive and/or bioresorbable nanoceramics as reinforcing agents that also add osteogenic and osteoconductive properties to these materials, making them potential and suitable candidates for the treatment of bone defects. For example, Saini and colleagues recently reported the preparation of a macroporous, 3D spongy scaffolds composed of alginate, gelatin and poly (vinyl alcohol) where nano-silver hydroxyapatite was incorporated into the 3D spongy scaffolds [59]. FTIR (Fourier transform infrared spectroscopy) revealed the presence of characteristic functional groups of alginate, gelatin, poly (vinyl alcohol), and silver hydroxyapatite in the scaffolds. The composite scaffolds were $80 \%$ porous with interconnected pores with sizes between 75 and $90 \mu \mathrm{m}$. The scaffolds showed antibacterial potential against Bacillus sp. and E.coli sp. and were not cytotoxic. It was observed a suppressed release of silver ions in simulated physiological fluids. These encouraging preliminary results warrant further investigation of these composite scaffolds for bone tissue engineering applications [59]. Using a factorial experimental design, Nabavinia and co-workers studied the influence of gelatin as a cell adhesive molecule and nHA as an osteoconductive component on the properties of alginate-based hydrogels and on the proliferation and osteogenic differentiation of microencapsulated osteoblast-like cells [60]. Results showed that nHA played a major role in promoting cell proliferation and osteogenic differentiation due to its bioactivity and contribution towards the improvement of the hydrogels' mechanical strength. The authors concluded that microcapsules with a composition of $1 \%$ alginate $/ 2.5 \%$ gelatin $/ 0.5 \%$ nHA, compressive modulus of $0.19 \mathrm{MPa} \pm 0.02$, swelling ratio of $52 \% \pm 8(24 \mathrm{~h})$ and degradation rate of $12 \% \pm 4(96 \mathrm{~h})$ displayed maximum cell proliferation and osteogenic differentiation, thus proposing a potential microcapsule composition as building blocks for modular bone tissue engineering [60].

As explained earlier in this chapter, fabrication of scaffolds for both tumour therapy and subsequent bone regeneration is a clinical need. Luo and colleagues recently proposed an injectable hydrogel of alginate and chitosan containing the chemotherapy drug cisplatin and polydopamine-decorated nHA. The hydrogel showed sustained release properties for cisplatin, effectively ablated tumour cells (4T1 cells) in vitro, and suppressed tumour growth in vivo. The injectable hydrogel also promoted in vitro adhesion and proliferation of bone mesenchymal stem cells [61].

Finally, in a last example of alginate-based hydrogels incorporating nBG, Rottensteiner-Brandl and co-workers encapsulated rat bone marrow derived mesenchymal stem cells (MSCs) into alginate dialdehyde/gelatin hydrogel with 
and without nBG. Results showed high cell survival in vitro for up to 28 days with or without nBG, thus proving the cellfriendly encapsulation process. After subcutaneous implantation into rats, high cell survival was observed 1 week after implantation; however, a notable decrease was seen after 4 weeks. The observed immune reaction was very mild, which proves the biocompatibility of the scaffold. Constructs incorporating nBG showed higher numbers of viable MSCs and lectin positive endothelial cells, thus showing higher angiogenesis. Nevertheless, this difference was not significant. After these promising results, the authors are now focusing on improving long term cell survival and differentiation potential of encapsulated cells in vivo [62,63].

3.4 Bioactive glass nanoceramics in skin repair and regeneration

Skin is the largest by weight and fastest-growing organ in the human body. It acts as thermoregulatory and sensory organ and also as a protective barrier. The skin comprises of two basic layers: the superficial thin epidermis $(0.1-0.15 \mathrm{~mm})$ is not vascularised and is continuously replaced through an organised differentiation process (cornification); on the other hand, the deeper thicker dermis $(1.5-3 \mathrm{~mm})$ is highly vascularised and contains appendages like sweat glands or hair follicles, playing a key role in thermoregulation, sensation, and healing. Wounds are formed when damage to the structure of skin occurs and they range from a simple epidermal cut to a deep dermal burn. Because of its complexity, injury to the dermis can lead to permanent impairment of function, especially in deep partial and full thickness wounds, which need urgent treatment with autologous skin grafts as the "gold standard". However, permanent damage to the skin at the donor site could occur leading to additional and sometimes severe scarring. Furthermore, donor sites are insufficient when dealing with large area burns. Thus, substantial research is being carried out to create alternative skin substitutes that avoid the problems just mentioned. In dermal substitutes, the majority of materials used in their development are polymers, both natural and synthetic. However, bioactive glasses have achieved notoriety in the last decade due to their ability to stimulate soft tissue regeneration[43].

Silicate-based bioglasses are the oldest bioactive glasses known and therefore were the first to be investigated for skin wound healing applications. For instance, 45S5 Bioglass ${ }^{\circledR}$ ionic extracts effectively promoted fibroblasts proliferation and migration as well as enhanced the secretion of collagen type I, thus accelerating wound healing [64]. More recently, the interest in borate-based bioactive glasses for skin repair has grown since it was shown that they can heal chronic wounds, although the toxicity of the released borate ions remains a concern [64]. Research into bioactive glasses for wound healing is relatively recent but offers great potential for enhancing healing of challenging wounds [64]. Nanosized bioactive glasses are also being investigated with interesting findings. For example, Gu and co-workers developed a new sol-gel-derived nBG powder material and evaluated its biological efficacy for skin repair based on the antibacterial and wound healing accelerating properties of the trace elements present in the material, which had an amorphous nature as confirmed by X-ray diffraction analysis. Biologically active ions (e.g. calcium, silicon, zinc, and boron) were rapidly released in Tris buffer at physiological temperature in a similar manner to the 45S5 Bioglass ${ }^{\circledR}(45 \mathrm{~S} 5 \mathrm{BG})$. In a rat model of deep second-degree scald, the nBG and 45S5 BG particles were well tolerated by the surrounding host wound tissue without causing any chronic inflammation, and appreciably enhanced the wound closure compared to 45S5 BG and the control (Fig. 4) [65]. 
A

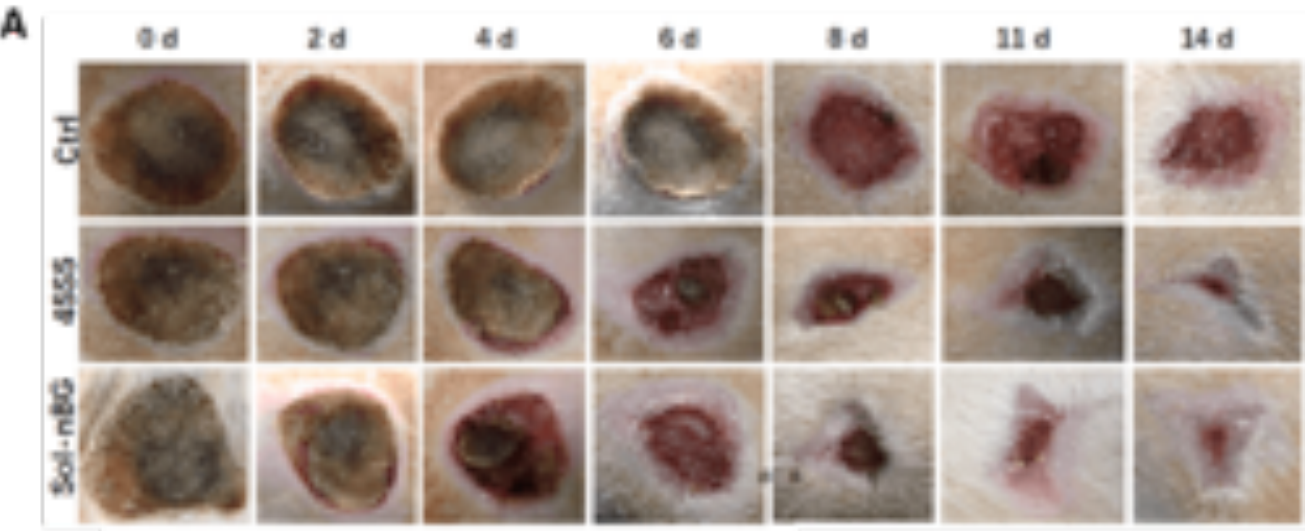

8

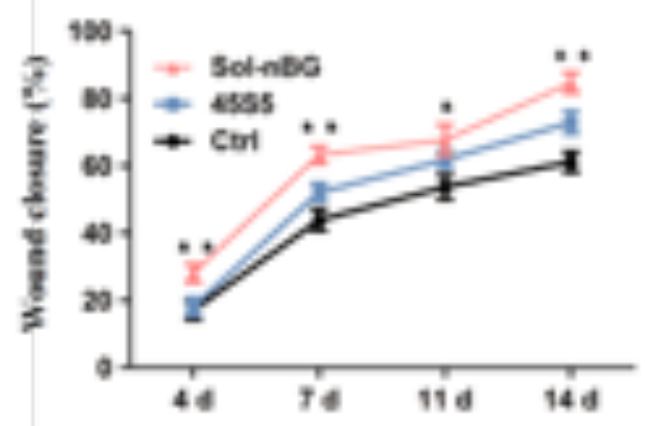

Figure 4. (A) Macrocopic images of the wound during healing process covered with and without BG products (B) wound closure percentage in the three groups $(* \mathrm{p}<0.05$, and $* * p<0.01$ as compared with control and $45 \mathrm{~S} 5 \mathrm{BG}$ groups) [65]. Reproduced from $\mathrm{Gu}$ et al. 2018; Int J Regen Med (Open Access article distributed under the terms of the Creative Commons Attribution License CC BY 4.0).

The strategy discussed throughout this chapter of dispersing nanoceramics in polymeric matrices have also been explored for wound healing applications. For instance, Chen and colleagues fabricated an electrospun nanofibrous membrane of chitosan/polyvinyl alcohol (PVA)/nBG with a spatially designed structure as a wound dressing for accelerating healing of chronic wounds. The membrane showed excellent biocompatibility, antibacterial activity and a regenerative promotion effect. In vivo experiments in rat full-thickness skin defects and mice diabetic chronic wounds showed that the membrane achieved complete re-epithelialization, improved collagen alignment, and formation of skin appendages by upregulating growth factors involved in wound healing such as vascular endothelial growth factor (VEGF) and transforming growth factor beta (TGF- $\beta$ ) while downregulating inflammatory cytokines like tumor necrosis factor alpha (TNF- $\alpha$ ) and interleukin 1 beta (IL-1 $\beta$ ) [66]. The work by Xu et al. is another example of the $\mathrm{nBG} /$ polymer strategy for wound healing applications. They reported the fabrication of a hierarchical electrospun scaffold with a micro-patterned and nano-sized fiber matrix, and surface-modified nBG. The scaffolds were firstly prepared by patterning electrospinning, and then pulsed laser deposition was applied for the preparation of the nBG layer deposited on the fibers' surface. The hierarchical micro/nano structure and presence of $\mathrm{nBG}$ in the scaffolds synergistically improved the efficiency and re-epithelialization in wounds created in the dorsal skin of mice [67]. These examples show the potential of combining nBG and polymeric matrices for wound healing appplications.

3.5 Bioactive glass nanoceramics in peripheral nerve regeneration

Peripheral nerves can suffer physical injuries caused by trauma leading to a significant loss of sensory or motor functions. Nerve regeneration can be achieved clinically with nerve guide conduits (NGC), a concept that has existed for more than a decade and has evolved to a clinical reality as an alternative to autologous nerve grafting. An ideal NGC should be biocompatible, biodegradable, permeable to allow nutrients and waste diffusion, mechanically robust while flexible, and electrically conductive. Researchers have used a biomaterial approach to build functional artificial NGC. One of the strategies used is to combine nBG and polymers, both natural and synthetic, of which some examples are given below.

Koudehi and colleagues developed a nBG/gelatin NGC with a pore size of 10-40 $\mu \mathrm{m}$. The NGC had good cytocompatibility in vitro. The guidance channel was examined and used to regenerate a $10 \mathrm{~mm}$ gap in the right sciatic nerve of 20 male Wistar rats that were randomly divided into two groups, with NGC and normal rats. Histological and functional evaluation indicated that at 3 months, nerve regeneration of the NGC group 
was statistically equivalent to the normal group. These results suggested that the $\mathrm{nBG}$ /gelatin NGC could be a suitable candidate for peripheral nerve repair[68]. After thorough in vitro testing [69], Mohamadi and colleagues also tested in a rat model a proposed electrospun nano-fibrous NGC made of polycaprolactone $(\mathrm{PCL})$, collagen and $\mathrm{nBG}$. The aim of Mohamadi et al.'s study was to evaluate sciatic nerve regeneration in a rat model after nerve transaction followed by human endometrial stem cells (hEnSCs) treatment into the NGC. Histology and immunohistochemistry results indicated that regenerative nerve fibres had been formed and were accompanied by blood vessels in the NGC/nEnSCs group. The authors concluded that the $\mathrm{PCL} /$ collagen/nBG nanofibrous NGC filled with hEnSCs was a suitable strategy to improve nerve regeneration after a nerve transaction in a rat model [70]. Finally, also using a combination of synthetic and natural polymers, Lin and colleagues fabricated by electrospinning a novel nerve conductor made of polypyrrole (PPY), collagen and nano-strontium substituted bioactive glass (nSrBG) (Fig. 5). Sciatic nerve deformity was evaluated in an animal model (rodents) with PPY/Collagen/nSrBG. NGC without nSrBG and autotransplants were used as controls. Compared with PPY/Collagen, PPY/Collagen/nSrBG group accomplished increasingly viable recovery of sciatic nerve wounds 24 weeks after implantation. The rejuvenated nerve filaments in the $\mathrm{PPY} /$ Collagen/nSrBG group had a round shape and the thickness of neuro-filaments was similar to that in the autotransplant control group [71].

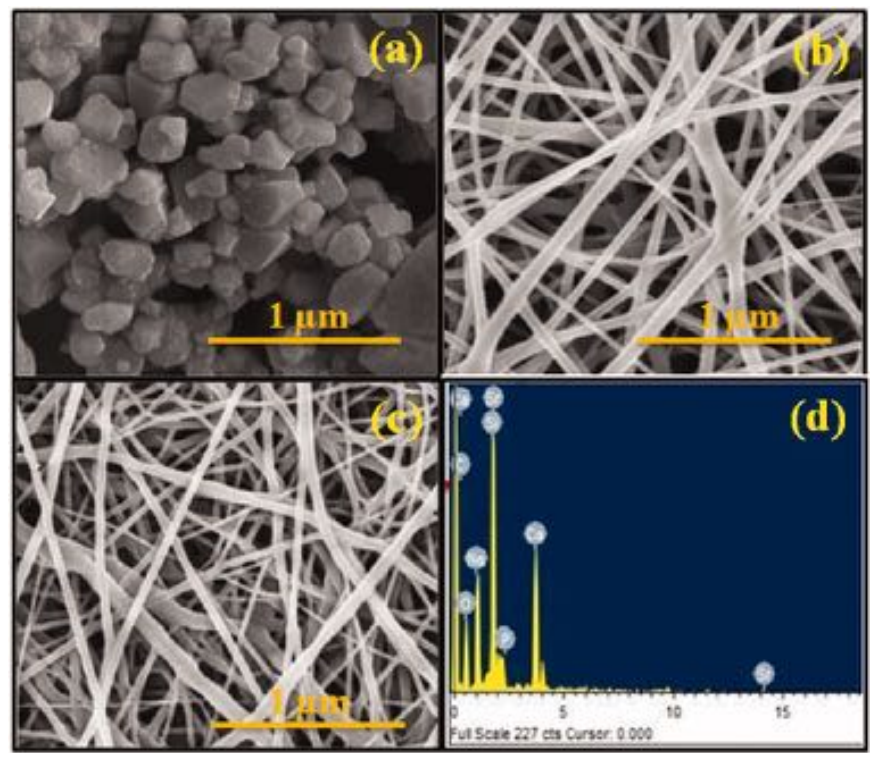

Figure 5. SEM images of (a) nSrBG, (b) electrospun nanofibers of PPY/Collagen, (c) electrospun nanofibers of $\mathrm{PPY} /$ Collagen/nSrBG, and (d) energy dispersive X-ray spectrum of PPY/Collagen/nSrBG composites clearly showing the presence of $\mathrm{Sr}, \mathrm{Ca}, \mathrm{P}, \mathrm{Si}, \mathrm{Na}, \mathrm{C}$ and O elements [71]. Reproduced from Lin et al. 2019; Artif Cells Nanomed Biotechnol (Open Access article distributed under the terms of the Creative Commons Attribution License CC BY 4.0).

\section{CONCLUSIONS}

In the fields of regenerative medicine and tissue engineering, the shortcomings of autografts and allografts have motivated the research of effective synthetic grafts. Particularly, nanotechnology applications have been extensively studied due to the nano-sized nature of the interactions between cells and the ECM of tissues. This chapter provides an overview of the use of bioactive and bioresorbable nanoceramics for regenerative medicine purposes, focusing on tissue engineering strategies where release of the nanoceramics has a therapeutic effect for healing and regeneration of various tissues. The strategy of dispersing nanoceramics in a polymeric matrix has the potential advantages of sustained release of nanoceramic particles, biomimicry when ECM-like polymers are used, and custom-fit implants when combined with additive manufacturing techniques.

\section{Acknowledgements}

This work was supported by the Restoration of Appearance and Function Trust (UK, Registered Charity No 299811) charitable funds. 


\section{References}

[1] Traykova T, Aparicio C, Ginebra MP, Planell JA. Bioceramics as nanomaterials. Nanomedicine $2006 ; 1$. https://doi.org/10.2217/17435889.1.1.91.

[2] Davison-Kotler E, Sharma V, Kang NV, García-Gareta E. A Universal Classification System of Skin Substitutes Inspired by Factorial Design. Tissue Eng Part B Rev 2018;24:279-88. https://doi.org/10.1089/ten.teb.2017.0477.

[3] García-Gareta E, Coathup MJ, Blunn GW. Osteoinduction of bone grafting materials for bone repair and regeneration. Bone 2015;81. https://doi.org/10.1016/j.bone.2015.07.007.

[4] Deng M, James R, Laurencin CT, Kumbar SG. Nanostructured polymeric scaffolds for orthopaedic regenerative engineering. IEEE Trans Nanobioscience 2012;11:3-14. https://doi.org/10.1109/TNB.2011.2179554.

[5] Jouybar A, Seyedjafari E, Ardeshirylajimi A, Zandi-Karimi A, Feizi N, Khani MM, et al. Enhanced Skin Regeneration by Herbal Extract-Coated Poly-L-Lactic Acid Nanofibrous Scaffold. Artif Organs 2017;41:E296307. https://doi.org/10.1111/aor.12926.

[6] Frohbergh ME, Katsman A, Botta GP, Lazarovici P, Schauer CL, Wegst UGK, et al. Electrospun hydroxyapatitecontaining chitosan nanofibers crosslinked with genipin for bone tissue engineering. Biomaterials 2012;33:9167-78. https://doi.org/10.1016/j.biomaterials.2012.09.009.

[7] Garcia-Gareta E, Hua J, Orera A, Kohli N, Knowles JC, Blunn GW. Biomimetic surface functionalization of clinically relevant metals used as orthopaedic and dental implants. Biomed Mater 2018;13. https://doi.org/10.1088/1748-605X/aa87e6.

[8] Traykova T, Aparicio C, Ginebra MP, Planell JA. Bioceramics as nanomaterials. Nanomedicine 2006;1:91-106. https://doi.org/10.2217/17435889.1.1.91.

[9] Qian X, Han X, Chen Y. Insights into the unique functionality of inorganic micro/nanoparticles for versatile ultrasound theranostics. Biomaterials 2017;142:13-30. https://doi.org/10.1016/J.BIOMATERIALS.2017.07.016.

[10] Kaur G, Pandey OP, Singh K, Homa D, Scott B, Pickrell G. A review of bioactive glasses: Their structure, properties, fabrication and apatite formation. J Biomed Mater Res Part A 2014;102:254-74. https://doi.org/10.1002/jbm.a.34690.

[11] Fu Q, Rahaman MN, Sonny Bal B, Brown RF, Day DE. Mechanical and in vitro performance of 13-93 bioactive glass scaffolds prepared by a polymer foam replication technique. Acta Biomater 2008;4:1854-64. https://doi.org/10.1016/J.ACTBIO.2008.04.019.

[12] Radin S, Reilly G, Bhargave G, Leboy PS, Ducheyne P. Osteogenic effects of bioactive glass on bone marrow stromal cells. J Biomed Mater Res Part A 2005;73A:21-9. https://doi.org/10.1002/jbm.a.30241.

[13] Yuan H, de Bruijn JD, Zhang X, van Blitterswijk CA de GK. Bone induction by porous glass ceramic made from Bioglass (45S5). J Biomed Mater Res 2001;58:270-6. https://doi.org/10.1002/1097-4636(2001)58:3<270::aidjbm1016>3.0.co;2-2.

[14] Hench LL. The story of Bioglass ${ }^{\circledR}$. J Mater Sci Mater Med 2006;17:967-78. https://doi.org/10.1007/s10856006-0432-z.

[15] Hoppe A, Güldal NS, Boccaccini AR. A review of the biological response to ionic dissolution products from bioactive glasses and glass-ceramics. Biomaterials 2011;32:2757-74. https://doi.org/10.1016/J.BIOMATERIALS.2011.01.004.

[16] LeGeros RZ. Calcium Phosphate-Based Osteoinductive Materials. Chem Rev 2008;108:4742-53. https://doi.org/10.1021/cr800427g.

[17] Sethu SN, Namashivayam S, Devendran S, Nagarajan S, Tsai W-B, Narashiman S, et al. Nanoceramics on osteoblast proliferation and differentiation in bone tissue engineering. Int J Biol Macromol 2017;98:67-74. https://doi.org/10.1016/j.ijbiomac.2017.01.089.

[18] Deepthi S, Venkatesan J, Kim S-K, Bumgardner JD, Jayakumar R. An overview of chitin or chitosan/nano ceramic composite scaffolds for bone tissue engineering. Int J Biol Macromol 2016;93:1338-53. https://doi.org/10.1016/j.ijbiomac.2016.03.041.

[19] Peter M, Binulal NS, Soumya S, Nair S V., Furuike T, Tamura H, et al. Nanocomposite scaffolds of bioactive glass ceramic nanoparticles disseminated chitosan matrix for tissue engineering applications. Carbohydr Polym 2010;79:284-9. https://doi.org/10.1016/j.carbpol.2009.08.001.

[20] Gong T, Xie J, Liao J, Zhang T, Lin S, Lin Y. Nanomaterials and bone regeneration. Bone Res 2015;3:15029. https://doi.org/10.1038/boneres.2015.29.

[21] Van Der Biest O. Nanoceramics: Issues and Opportunities 2013. https://doi.org/10.1111/ijac.12074.

[22] Sharma CP, Paul W. Nanoceramic Matrices: Biomedical Applications. Am J Biochem Biotechnol 2006;2:41-8.

[23] de Siqueira L, Gouveia RF, Grenho L, Monteiro FJ, Fernandes MH, Trichês ES. Highly porous $45 \mathrm{~S} 5$ bioglassderived glass-ceramic scaffolds by gelcasting of foams. J Mater Sci 2018;53:10718-31. https://doi.org/10.1007/s10853-018-2337-x.

[24] Kiani A, Rahmani M, Manickam S, Tan B. Nanoceramics: Synthesis, characterization, and applications. J 
Nanomater 2014;2014:2-4. https://doi.org/10.1155/2014/528348.

[25] Yang L. Nanotechnology-enhanced orthopedic materials : fabrications, applications and future trends. Woodhead Publishing; 2015.

[26] Yang L. Orthopedic nanoceramics. Nanotechnology-Enhanced Orthop. Mater., Woodhead Publishing; 2015, p. 49-75. https://doi.org/10.1016/B978-0-85709-844-3.00003-3.

[27] Hong Y, Fan H, Li B, Guo B, Liu M, Zhang X. Fabrication, biological effects, and medical applications of calcium phosphate nanoceramics. Mater Sci Eng R Reports 2010;70:225-42.

https://doi.org/10.1016/j.mser.2010.06.010.

[28] Chavan PN, Bahir MM, Mene RU, Mahabole MP, Khairnar RS. Study of nanobiomaterial hydroxyapatite in simulated body fluid: Formation and growth of apatite. Mater Sci Eng B Solid-State Mater Adv Technol 2010;168:224-30. https://doi.org/10.1016/j.mseb.2009.11.012.

[29] Nikam A V., Prasad BLV, Kulkarni AA. Wet chemical synthesis of metal oxide nanoparticles: A review. CrystEngComm 2018;20:5091-107. https://doi.org/10.1039/C8CE00487K.

[30] Gan L, Pilliar R. Calcium phosphate sol-gel-derived thin films on porous-surfaced implants for enhanced osteoconductivity. Part I: Synthesis and characterization. Biomaterials 2004;25:5303-12. https://doi.org/10.1016/j.biomaterials.2003.12.038.

[31] Eliaz N, Metoki N. Calcium Phosphate Bioceramics: A Review of Their History, Structure, Properties, Coating Technologies and Biomedical Applications. Materials (Basel) 2017;10. https://doi.org/10.3390/MA10040334.

[32] Fellah BH, Layrolle P. Sol-gel synthesis and characterization of macroporous calcium phosphate bioceramics containing microporosity. Acta Biomater 2009;5:735-42. https://doi.org/10.1016/J.ACTBIO.2008.09.005.

[33] Vichery C, Nedelec J-M. Bioactive Glass Nanoparticles: From Synthesis to Materials Design for Biomedical Applications. Materials (Basel) 2016;9:288. https://doi.org/10.3390/ma9040288.

[34] Samavedi S, Whittington AR, Goldstein AS. Calcium phosphate ceramics in bone tissue engineering: A review of properties and their influence on cell behavior. Acta Biomater 2013;9:8037-45. https://doi.org/10.1016/J.ACTBIO.2013.06.014.

[35] Baino F, Hamzehlou S, Kargozar S. Bioactive Glasses: Where Are We and Where Are We Going? J Funct Biomater 2018;9:25. https://doi.org/10.3390/jfb9010025.

[36] Kaur G, Pickrell G, Sriranganathan N, Kumar V, Homa D. Review and the state of the art: Sol-gel and melt quenched bioactive glasses for tissue engineering. J Biomed Mater Res - Part B Appl Biomater 2016;104:124875. https://doi.org/10.1002/jbm.b.33443.

[37] Wang M, Guo L, Sun H. Manufacture of Biomaterials. Encycl Biomed Eng 2019:116-34. https://doi.org/10.1016/B978-0-12-801238-3.11027-X.

[38] Basu B, Katti D, Kumar A. Advanced biomaterials : fundamentals, processing, and applications. John Wiley \& Sons; 2009.

[39] Covarrubias C, Arroyo F, Balanda C, Neira M, Von Marttens A, Caviedes P, et al. The Effect of the Nanoscale Structure of Nanobioceramics on Their In Vitro Bioactivity and Cell Differentiation Properties. J Nanomater 2015;2015:1-14. https://doi.org/10.1155/2015/526230.

[40] Kokubo T, Kushitani H, Sakka S, Kitsugi T, Yamamum T. Surface-Structure Changes in Bioactive 1990;24:72134.

[41] Lin K, Chang J. Structure and properties of hydroxyapatite for biomedical applications. Hydroxyapatite Biomed Appl 2015:3-19. https://doi.org/10.1016/B978-1-78242-033-0.00001-8.

[42] Ebrahimi M, Botelho M, Lu W, Monmaturapoj N. Synthesis and characterization of biomimetic bioceramic nanoparticles with optimized physicochemical properties for bone tissue engineering. J Biomed Mater Res Part A 2019;107:jbm.a.36681. https://doi.org/10.1002/jbm.a.36681.

[43] García-Gareta E. Collagen, from Tissue Culture to Biomaterials, Tissue Engineering, and Beyond. First edition. Cambridge Scholars Publishing; 2019.

[44] Marelli B, Ghezzi CE, Mohn D, Stark WJ, Barralet JE, Boccaccini AR, et al. Accelerated mineralization of dense collagen-nano bioactive glass hybrid gels increases scaffold stiffness and regulates osteoblastic function. Biomaterials 2011;32:8915-26. https://doi.org/10.1016/J.BIOMATERIALS.2011.08.016.

[45] Hafezi Foroughand Hosseinnejad F and FAAI and MMS and AA and NMR. Transplantation of nanobioglass/gelatin scaffold in a non-autogenous setting for bone regeneration in a rabbit ulna. J Mater Sci Mater Med 2012;23:2783-92. https://doi.org/10.1007/s10856-012-4722-3.

[46] Maji K, Dasgupta S, Pramanik K, Bissoyi A. Preparation and Evaluation of Gelatin-Chitosan-Nanobioglass 3D Porous Scaffold for Bone Tissue Engineering. Int J Biomater 2016;2016:9825659. https://doi.org/10.1155/2016/9825659.

[47] Wang L, Xu L, Peng C, Teng G, Wang Y, Xie X, et al. The effect of bone marrow mesenchymal stem cell and nano-hydroxyapatite/collagen I/poly-L-lactic acid scaffold implantation on the treatment of avascular necrosis of the femoral head in rabbits. Exp Ther Med 2019;18:2021-8. https://doi.org/10.3892/etm.2019.7800. 
[48] Liu H-C, E L-L, Wang D-S, Su F, Wu X, Shi Z-P, et al. Reconstruction of Alveolar Bone Defects Using Bone Morphogenetic Protein 2 Mediated Rabbit Dental Pulp Stem Cells Seeded on Nano-

Hydroxyapatite/Collagen/Poly(L-lactide). Tissue Eng Part A 2011;17:2417-33.

https://doi.org/10.1089/ten.tea.2010.0620.

[49] Rong Zi-Jieand Yang L-J and CB-T and ZL-X and CY-L and WG-F and ZZ-J. Porous nano-hydroxyapatite/collagen scaffold containing drug-loaded ADM-PLGA microspheres for bone cancer treatment. J Mater Sci Mater Med 2016;27:89. https://doi.org/10.1007/s10856-016-5699-0.

[50] Forero JC, Roa E, Reyes JG, Acevedo C, Osses N. Development of Useful Biomaterial for Bone Tissue Engineering by Incorporating Nano-Copper-Zinc Alloy (nCuZn) in Chitosan/Gelatin/Nano-Hydroxyapatite (Ch/G/nHAp) Scaffold. Materials (Basel) 2017;10. https://doi.org/10.3390/ma10101177.

[51] Lee JS, Baek SD, Venkatesan J, Bhatnagar I, Chang HK, Kim HT, et al. In vivo study of chitosan-natural nano hydroxyapatite scaffolds for bone tissue regeneration. Int J Biol Macromol 2014;67:360-6. https://doi.org/10.1016/j.ijbiomac.2014.03.053.

[52] Ma X, Wang Y, Guo H, Wang J. Nano-hydroxyapatite/chitosan sponge-like biocomposite for repairing of rat calvarial critical-sized bone defect. J Bioact Compat Polym 2011;26:335-46. https://doi.org/10.1177/0883911511407402.

[53] Lowe B, Venkatesan J, Anil S, Shim MS, Kim SK. Preparation and characterization of chitosan-natural nano hydroxyapatite-fucoidan nanocomposites for bone tissue engineering. Int J Biol Macromol 2016;93:1479-87. https://doi.org/10.1016/j.ijbiomac.2016.02.054.

[54] Pangon A, Saesoo S, Saengkrit N, Ruktanonchai U, Intasanta V. Hydroxyapatite-hybridized chitosan/chitin whisker bionanocomposite fibers for bone tissue engineering applications. Carbohydr Polym 2016;144:41927. https://doi.org/10.1016/j.carbpol.2016.02.053.

[55] Tripathi A, Saravanan S, Pattnaik S, Moorthi A, Partridge NC, Selvamurugan N. Bio-composite scaffolds containing chitosan/nano-hydroxyapatite/nano-copper-zinc for bone tissue engineering. Int J Biol Macromol 2012;50:294-9. https://doi.org/10.1016/j.ijbiomac.2011.11.013.

[56] Sowmya S, Sudheesh Kumar PT, Chennazhi KP, Nair S V, Tamura H, Jayakumar R. Biocompatible $\beta \beta \beta \beta \beta-$ chitin Hydrogel/Nanobioactive Glass Ceramic Nanocomposite Scaffolds for Periodontal Bone Regeneration. vol. 25. 2011.

[57] Zhang Y, Zhou D, Chen J, Zhang X, Li X, Zhao W, et al. Biomaterials Based on Marine Resources for 3D Bioprinting Applications. Mar Drugs 2019;17. https://doi.org/10.3390/md17100555.

[58] Venkatesan J, Bhatnagar I, Manivasagan P, Kang K-H, Kim S-K. Alginate composites for bone tissue engineering: A review. Int J Biol Macromol 2015;72:269-81. https://doi.org/10.1016/J.IJBIOMAC.2014.07.008.

[59] Kumar Saini R, Prasad Bagri L, Bajpai AK. Nano-silver hydroxyapatite based antibacterial 3D scaffolds of gelatin/alginate/poly (vinyl alcohol) for bone tissue engineering applications. Colloids Surfaces B Biointerfaces 2019;177:211-8. https://doi.org/10.1016/J.COLSURFB.2019.01.064.

[60] Nabavinia M, Khoshfetrat AB, Naderi-Meshkin H. Nano-hydroxyapatite-alginate-gelatin microcapsule as a potential osteogenic building block for modular bone tissue engineering. Mater Sci Eng C 2019;97:67-77. https://doi.org/10.1016/J.MSEC.2018.12.033.

[61] Luo S, Wu J, Jia Z, Tang P, Sheng J, Xie C, et al. An Injectable, Bifunctional Hydrogel with Photothermal Effects for Tumor Therapy and Bone Regeneration. Macromol Biosci 2019;19:1900047. https://doi.org/10.1002/mabi.201900047.

[62] Rottensteiner U, Sarker B, Heusinger D, Dafinova D, Rath SN, Beier JP, Kneser U, Horch RE, Detsch R, Boccaccini AR AA. In vitro and in vivo Biocompatibility of Alginate Dialdehyde/Gelatin Hydrogels with and without Nanoscaled Bioactive Glass for Bone Tissue Engineering Applications. Mater 2014;7. https://doi.org/10.3390/ma7031957.

[63] Rottensteiner-Brandl U, Detsch R, Sarker B, Lingens L, Köhn K, Kneser U, Bosserhoff AK, Horch RE, Boccaccini AR AA. Encapsulation of Rat Bone Marrow Derived Mesenchymal Stem Cells in Alginate Dialdehyde/Gelatin Microbeads with and without Nanoscaled Bioactive Glass for In Vivo Bone Tissue Engineering. Mater $2018 ; 11$. https://doi.org/10.3390/ma11101880.

[64] Li H, Wu Z, Zhou Y, Chang J. 8 - Bioglass for skin regeneration. In: García-Gareta E, editor. Biomater. Ski. Repair Regen., Woodhead Publishing; 2019, p. 225-50. https://doi.org/https://doi.org/10.1016/B978-0-08-1025468.00008-X.

[65] Gu W, Li Y, Yang X, Jin Z, Shen J, Gou Z. Science Repository Systematic investigation of a new nanoscale bioactive glass on wound healing in vivo in comparison with the clinically applied 45S5 Bioglass @ 2018. https://doi.org/10.31487/j.RGM.2018.10.001.

[66] Chen Q, Wu J, Liu Y, Li Y, Zhang C, Qi W, et al. Electrospun chitosan/PVA/bioglass Nanofibrous membrane with spatially designed structure for accelerating chronic wound healing. Mater Sci Eng C 2019;105:110083. https://doi.org/10.1016/J.MSEC.2019.110083. 
[67] Xu H, Lv F, Zhang Y, Yi Z, Ke Q, Wu C, et al. Hierarchically micro-patterned nanofibrous scaffolds with a nanosized bio-glass surface for accelerating wound healing. Nanoscale 2015;7:18446-52. https://doi.org/10.1039/C5NR04802H.

[68] Koudehi Masoumeh Foroutanand Fooladi AAI and MK and JZ and AA and NMR. Preparation and evaluation of novel nano-bioglass/gelatin conduit for peripheral nerve regeneration. J Mater Sci Mater Med 2014;25:36373. https://doi.org/10.1007/s10856-013-5076-1.

[69] Mohamadi F, Ebrahimi-Barough S, Reza Nourani M, Ali Derakhshan M, Goodarzi V, Sadegh Nazockdast M, et al. Electrospun nerve guide scaffold of poly( $\varepsilon$-caprolactone)/collagen/nanobioglass: an in vitro study in peripheral nerve tissue engineering. J Biomed Mater Res Part A 2017;105:1960-72. https://doi.org/10.1002/jbm.a.36068.

[70] Mohamadi F, Ebrahimi-Barough S, Nourani MR, Mansoori K, Salehi M, Alizadeh AA, et al. Enhanced sciatic nerve regeneration by human endometrial stem cells in an electrospun poly ( $\varepsilon$-caprolactone)/collagen/NBG nerve conduit in rat. Artif Cells, Nanomedicine, Biotechnol 2018;46:1731-43. https://doi.org/10.1080/21691401.2017.1391823.

[71] Lin B, Dun G, Jin D, Du Y. Development of polypyrrole/collagen/nano-strontium substituted bioactive glass composite for boost sciatic nerve rejuvenation in vivo. Artif Cells, Nanomedicine, Biotechnol 2019;47:3423-30. https://doi.org/10.1080/21691401.2019.1638794. 


\title{
CHAPTER 11 \\ NANOSAFETY ISSUES
}

Fabio Pizzetti a, and Giuseppe Perale

a Department of Chemistry, Materials and Chemical Engineering "G. Natta”, Politecnico di Milano, 20133 Milan, Italy;

b Faculty of Biomedical Sciences, University of Southern Switzerland (USI), Via Buffi 13, 6900, Lugano, Switzerland;

c Ludwig Boltzmann Institute for Experimental and Clinical Traumatology, Donaueschingenstrasse 13, 1200 Vienna, Austria.

* corresponding author: giuseppe.perale@usi.ch

\begin{abstract}
The Safe-by-Design ( $\mathrm{SbD}$ ) approach is a general concept used in order to identify risks and uncertainties involved in human health and environmental safety during the initial stages of the development of a product. Here this approach is applied to polymeric nanobiomaterials used in the nanomedicine framework. Initially, a brief introduction on NBMs will be given, including information on the current state of science and some gaps or uncertainties. Then, guidelines for implementing a $\mathrm{SbD}$ approach will be given, focus the attention on risks and hazard affecting such a material. The goals of the guidelines will be to:
\end{abstract}

- Support informed decision-making in the field of polymeric NBMs for drug delivery,

- Improve and facilitate communication between the different stakeholders and between industry and regulatory authorities,

- Prevent misguided investment,

- Enable small and medium-sized enterprises to deliver safe products in a competitive market. 


\subsection{MATERIAL DESIGN}

Nanobiomaterials (NBMs) are, in medicine, nanoscale materials able to give a response for a drug in a specific application. Unfortunately, a unique definition does not exist, since it changes according to the regulatory authorities around the world. The usable materials range from polymeric and organic networks to inorganic particles. Here the focus will be centered on polymeric NBMs since they have interesting characteristics for drug delivery. Polymers can be either natural or synthetic; usually natural ones show stability problems in biological media and difficult reproducibility. Synthetic polymers can be produced through chemical modification of natural ones, such as the mostly used poly-(D,L-lactide). The selection of the polymer to be used for a certain biomedical application depends on several factors, such as toxicity, biocompatibility, drug release profile, solubility and stability of the incapsulated drug and other physicochemical properties. One of the most important properties is the biodegradability since it determines the mechanism by which it is removed from the body. When designing polymeric NBMs the important part is that of tailoring their physicochemical properties according to the end usage. NBMs used as drug delivery systems should be able to control the delivery of the charged drug and remain stable throughout their shelf-life. Several factors influence NBMs design, such as the type of disease and drug, the route of administration, cells to be targeted, drug release kinetics and the dose. As far as the administration route is concerned, several possibilities exist, and they can be classified in oral, parenteral, respiratory and transdermal routes. Figure 1 presents an example of a decision tree taking into account these differences. Once the body has been entered, the NBMs should cross several barriers before reaching the site of action; this can be accomplished through passive diffusion and by active targeting. In order to have the active targeting method, there is the need of having certain targeting moieties or small molecules, able to guarantee a specific interaction with the target-cell membranes and trigger cellular uptake. Furthermore, such a nanocarrier should be able to deliver the drug to the specific site of action and should also release the drug at a rate able to ensure a therapeutic effect. 
A key factor for the efficacy and safety of polymeric NBMs is their interaction with physiological environment. As an example, every interaction with the immune system should be avoided. Usually NBMs, when used for drug delivery purposes, are administered parenterally. As soon as they enter the bloodstream their surface start to be surrounded by plasma components and forming a protein corona. The composition and the kinetics of formation of such a corona determine the biological identity of the NBMs. The protein corona is not a stable surface, since also the first protein adsorbed are later replaced by other ones. Unfortunately, the protein adsorption on the NBMs surface can be recognized by the immune system, starting an immune reaction (opsonization). It is thus important to design nanocarriers able to avoid opsonization and this can be done with functionalization of NBMs surface. PEGylation is probably the most widely used techniques, where opsonization is avoided by increasing the surface hydrophilicity. The same effect can be also obtained by steric hindrance induced by the PEG chains on the NBM surface. 


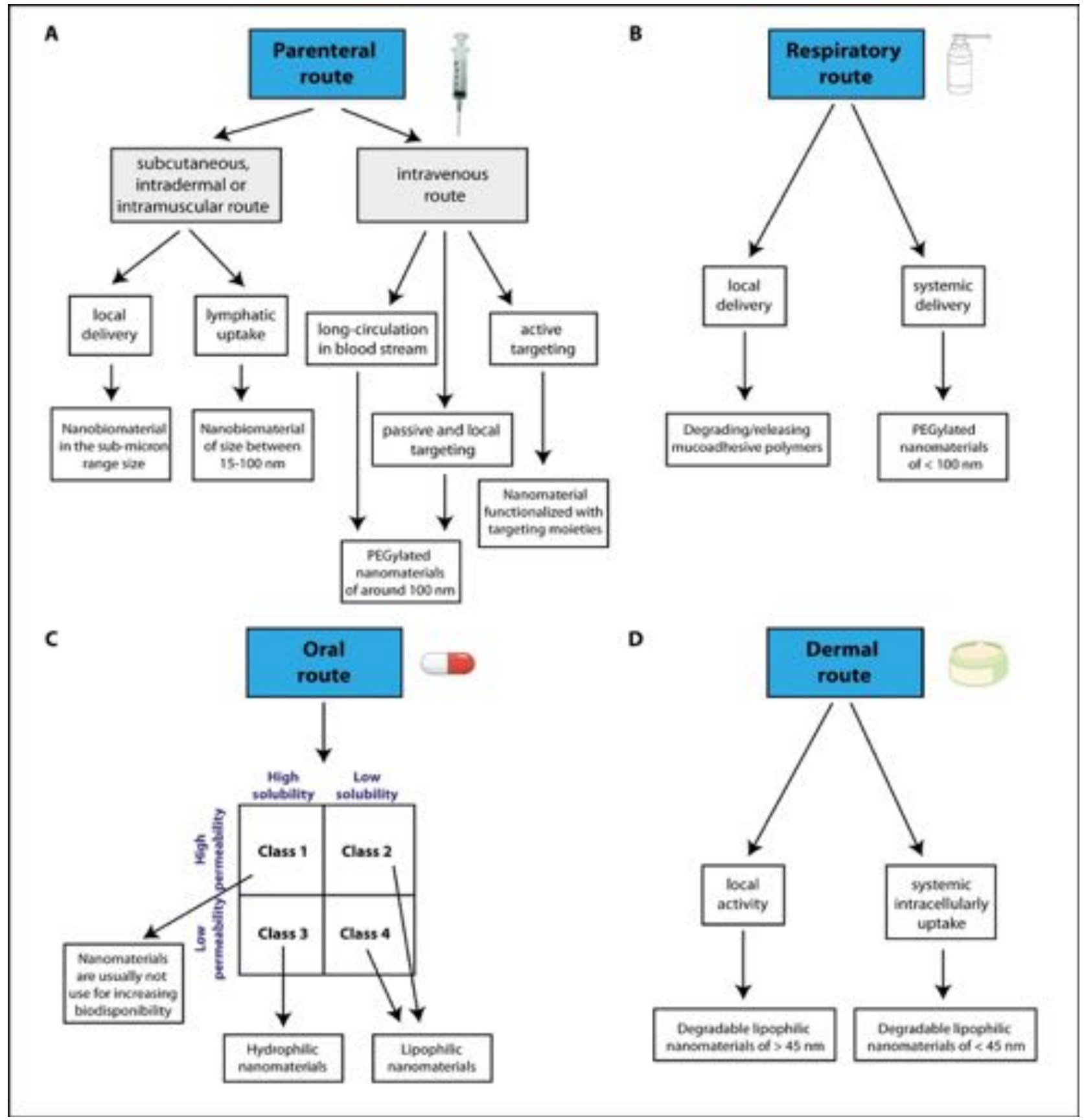

Figure 5 - Decision tree for choosing a nanobiomaterial taking into account the various factors discussed in this chapter.

Shifting to safety, it should not only be evaluated from the drug-nanocarrier, but also from the nanocarrier itself. NBM toxicity can be screened through literature review (if such a material has been widely used) or using "non-testing tools". As far as the second one is concerned, their use have been suggested in order to reduce the need for animal testing. 
What is usually used for evaluating the toxicity and biological interface interactions of NBMs different techniques are used:

- (Quantitative) Structure-Activity-Relationship or (Q)SAR. It is a regression analysis often used for drug discovery, its aim is that of finding a relation between NBM properties and the desired activity. The model will give a numerical prediction which will be able to asses if a certain material is safe for medical applications.

- Grouping and Read-Across. The goal is that of filling in data gaps, initially by having groupings of NBM properties and/or effects and then through interpolation for missing data. Here the idea is that similar materials show similar properties. Such an interpolation allows to predict a certain material endpoint if data are not available.

- Molecular modelling. These techniques are powerful tools for predicting the interactions between polymer surfaces and small or macro molecules. Unfortunately, due to computational limitations, the maximum nanoparticles size is confined to 10 to $20 \mathrm{~nm}$. Molecular modelling is a complementary tool to laboratory activities, since it can be used to understanding some complex interactions. However, it cannot completely replace lab activities and it can not be used as a merely prediction tool.

\subsection{REGULATORY FRAMEWORK}

The first step toward the development of a nanomedicine is the understanding of the relevant regulatory framework and their requirements. The principal goals of medicine regulations are to ensure safety, efficacy and quality of new medicines. Thus, any potential risks associated to such medicine should be eliminated or mitigated. Unfortunately, there is not a global set of regulations. Indeed, they change with the regions and with the medicine applications, thus attention should be paid on this topic. 
Nanomedicine is defined as "the medical application of nanotechnology" and can be divided in:

- Nanocarriers for drug delivery and nanopharmaceuticals,

- Medical devices,

- In vitro and in vivo diagnostics.

In the following, the focus will be centered on nanopharmaceutics, and few information on medical devices will also be given. The third part is out of the scope, thus will not be discussed anymore. As far as now, there is no distinction in the regulations between nanocarriers for drug delivery and conventional medicines. However, the authorities may have the possibility of asking for nano-specific questions. Shifting to the regulations for medical devices, probably the use of nanomaterials could require a more specific classification depending on the risk of internal exposure, hence requiring clinical trials. Recently, FDA published a draft guidance, listing a series of factors that should be considered for safety, efficacy and quality in the development of a nanomedicine, such as:

- The adequacy between the characterization of a material and its function,

- The structure complexity,

- The understanding of the relation between the material physicochemical properties and its biological effect,

- The understanding of the in vivo release mechanism correlated with the material physicochemical properties,

- The predictability of the in vivo release from the in vitro release tests,

- Stability (both physical and chemical),

- Maturity of the nanotechnology involved,

- The impact of manufacturing change on the drug quality,

- The nanomaterial physical state at administration and its route of administration,

- The material's bioavailability, biodegradability, accumulation, distribution and dissolution and their predictability from physicochemical properties and animal studies. 
As far as nanocarriers are concerned, their registration with the relevant authority request a complete set of pre-clinical and clinical studies, since they are considered new drugs, even if the drug or the nanocarrier have been already been approved before. The idea behind this is that nanocarriers are able to change drugs bioavailability, for instance changing their pharmacokinetics and/or pharmacodynamics, thus having an impact on their safety. Thanks to their complex structure, drug loaded nanocarriers are considered non-biological complex drugs (NBCDs). Similar to their biological counterparts, NBCDs can not be fully characterized, therefore the manufacture and registration of follow-on drug nanomedicines seems to be impossible. Despite such nanomedicine follow-on products have already received an authorization in the past, discussions among stakeholders are currently ongoing in order to introduce a regulatory strategy for "nanosimilars". This will probably be a further impediment in the development and marketing of future follow-on nanomedicines. The European Commission's conformity assessments for medical devices consisting of NBMs or with a nanoscale coating are identical to those for conventional medical devices. This has the meaning that the conformity is only dependent on the type of medical device, which for devices incorporating NBMs are classes III, IIa or IIb. Nevertheless, the certified body responsible for the European Commission of conformity should be accredited for the certification of devices that incorporates or consist of NBMs. Unfortunately, for medical devices, this revision process could be complicated, due to the limited availability of accredited notified bodies for medical devices of all classes. Just to make an example, now only 57 notified bodies exist in the European Union and affiliated countries, while few years ago they were more than 80 . This number is also thought to decrease even more as this is the current trend correlated to EU regulations 2017/745 and 2017/746. Similarly to traditional pharmaceutics, the development of a successful nanopharmaceutical require an adherence to strict quality-system regulations. During the preclinical studies, all the tests should be done under the principle of GLP, or Good Laboratory Practice, developed in accordance with the OECD (Organization for Economic Cooperation and Development). 
These rules concern the organization and the condition under with non-clinical studies are planned, performed, monitored, recorded, archived and reported. Furthermore, they ensure quality and validity of data collected in this initial phase. After the preclinical studies but before entering the clinical tests, a nanopharmaceutical should be manufactured according to the principles of GMP, or Good Manufacturing Practice. This aspect should be theoretically considered even in earlier phases, since the collaboration with the manufacturer should begin as soon as possible in order to ensure good quality in the clinical trials. Furthermore, GMP is also the standard for meeting the requirements of a marketing authorization, or MA. Other than GMP, a nanopharmaceutical must also be agreed by an Ethics Committee before entering the clinical tests phase. Moreover, the clinical phases must follow the standards of GCP, or Good Clinical Practice. Here it is ensured that the rights and the safety of the trial's participants are protected and also that the data produced and collected in these phases are reliable. Medical devices, instead, must meet the standards of the ISO 13485:2016 quality management system. Are there any nano-specific guidelines? The answer to this question is yes, different organizations have drafted guidelines in order to help companies in the development of a medicinal product. Some example will now be listed:

- EMA: The European Medicines Agency has created guidelines on nanomedicine for helping the developers to produce MA applications for human medicines.

- FDA: The US Food and Drug Administration established a guidance document, including information on drug products that contain nanomaterials.

- ICH: The International Conference on Harmonization has created a series of guidelines listing quality, efficacy and safety.

- OECD: Their guidelines enable the assessment of the potential effects of chemicals on human health and environment. Furthermore, it has also produced a Guidance Manual containing information for GMP. 
- SCENIHR: The European Commission's Scientific Committee on Emerging and Newly Identified Health Risks has established a series of guidelines on the potential effects of nanomaterials used in medical devices.

Also for polymers the regulations are different depending on the type of application and the countries where they will be marketed. In Figures 2-4 some case studies of different decision pathways under various conditions can be observed. It should be noted that, in Switzerland and EU, polymers used only for therapeutic products are exempt from notification, in Switzerland, and registration, in EU, because these products are regulated by other regulations.

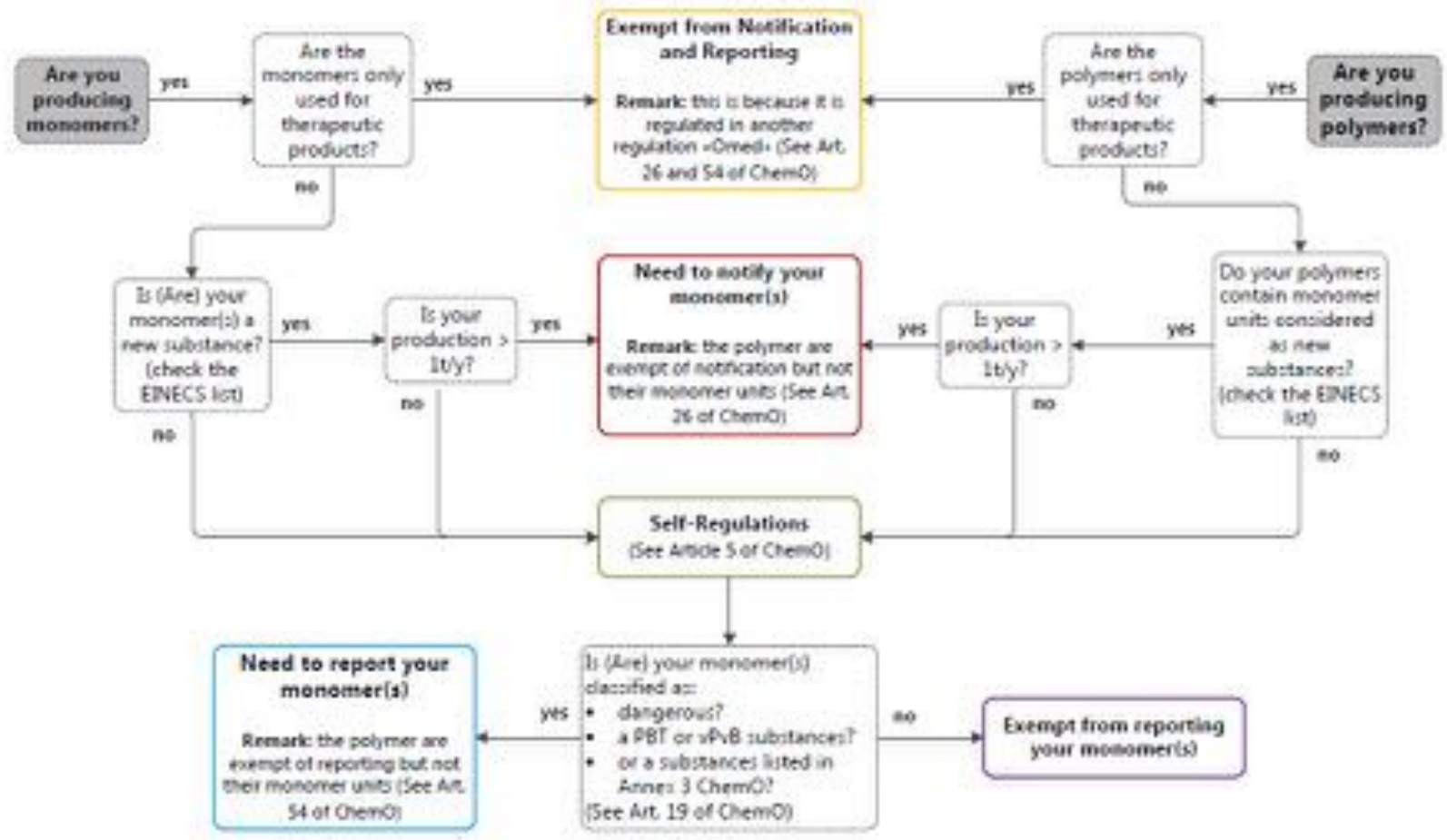

Figure 6 - Decision tree for companies producing monomers or polymers in Switzerland. 


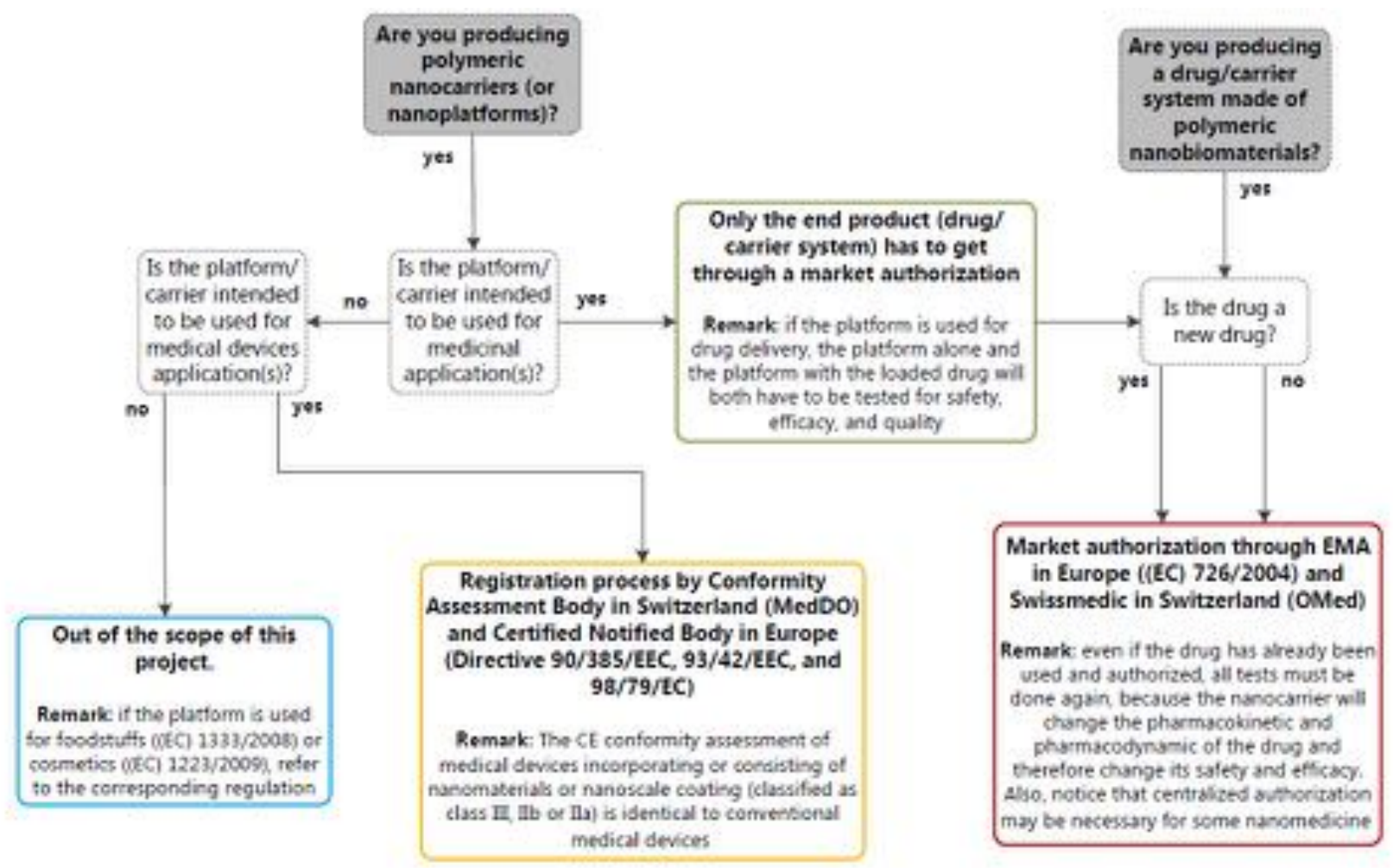

Figure 7 - Decision tree for companies producing monomers or polymers in Europe.

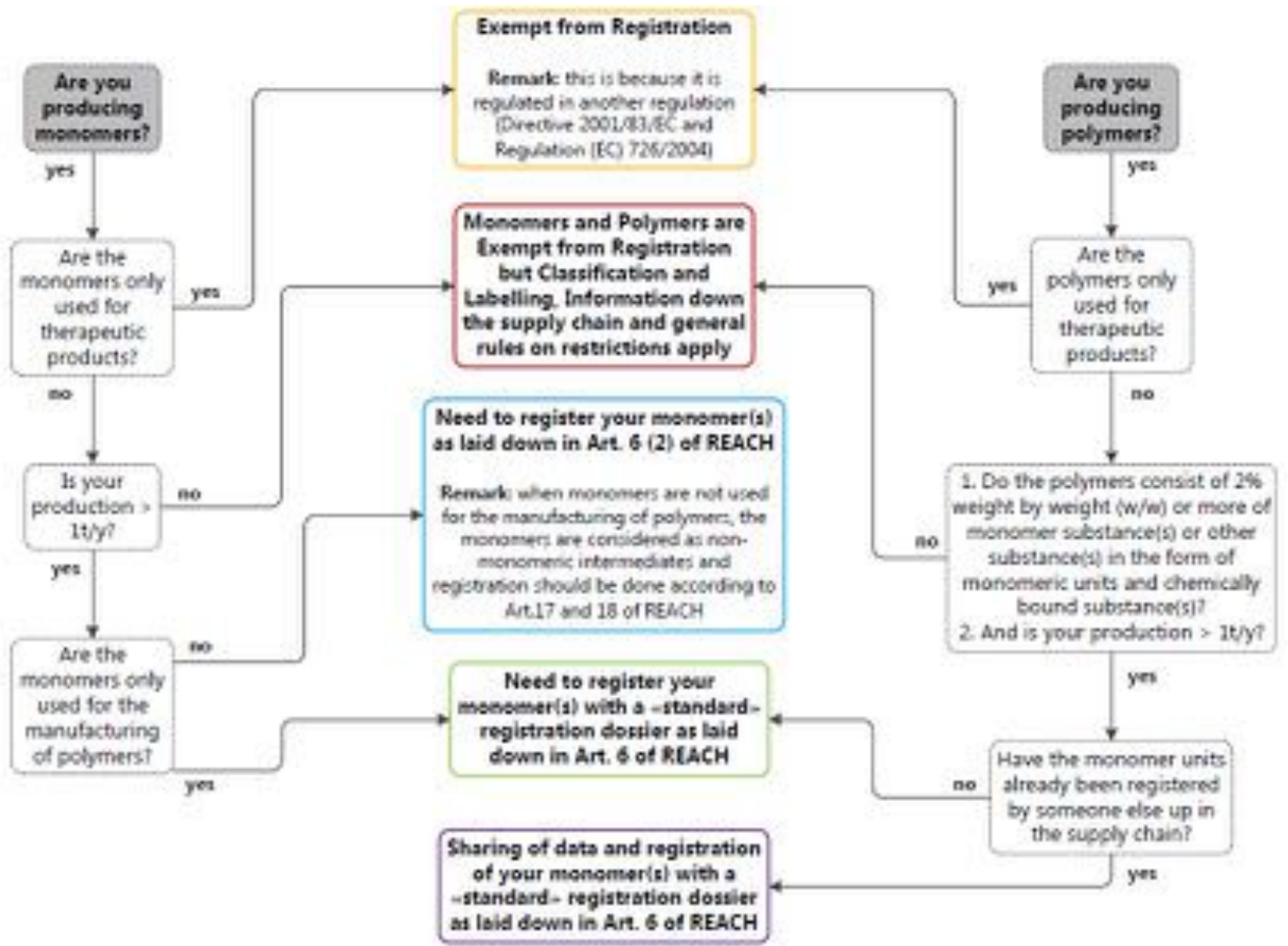

Figure 8 - Decision tree for companies producing either polymeric nanoplatform or drug/nanocarrier system made of polymeric nanobiomaterials in Switzerland and Europe. 


\subsection{HUMAN HEALTH RISKS OF POLYMERIC NANOBIOMATERIALS}

As already explained, the versatile physicochemical properties of polymeric NBMs make them interesting as novel drug nanocarriers. It is thus essential to evaluate the potential human health risks of such a material. In the nanomedicine field, two different types of exposure can be distinguished: intended exposure via patient administration and unintended exposure. Exposure assessment are defined by different factors, such as the type of administration, the dose and the treatment duration. Even if the in the case of patient exposure the scenarios are well defined, it is the opposite for the unintended exposures, since they can happen in a variety of possibilities, resulting also in a possible cumulative level of exposure and accumulations in non-target organs, where the impact on human health might be different from the one predicted. Furthermore, even the workers exposure on empty nanocarriers should be taken into consideration, since they can show different effect from the final formulation. Every polymer's properties should thus be evaluated, such as the molecular weight, chemical modification, purity but also the final dimension of the NBM, its $\zeta$-potential and its surface chemistry. A complete NBM hazard characterization should thus include the qualitative and quantitative description of all the possible toxicological effects observed in in vitro and in vivo toxicology studies, showing, if possible, the effect of under and overdosing. As far as the exposure is concerned, different administration routes should be taken into consideration: respiratory, oral, ocular, dermal and parenteral. Each route is characterized by its own biodistribution pattern, thus having different effects on human health. The pharmacokinetics is influenced by the polymeric NBM administration route. Moreover, when used as drug delivery systems, also their physicochemical properties such as size, chemistry and surface charge show a major influence on the pharmacokinetics of the drug to be delivered. As a matter of fact, polymeric NBMs are able to increase the absorption of low-bioavailability drugs by promoting their dissolution or by increasing their half-life, thus enhancing the therapeutic efficacy. Unfortunately, this could also increase the drug's original toxicity profile. 
The pharmacokinetics of both drug and drug loaded nanocarriers are important to understand and predict the final efficacy and toxicity, thus EMA has recommended the evaluation and the comparison of the pharmacokinetics of the final formulation but also of the drug alone. As an example, a list on how some NBMs properties affect the pharmacokinetics and the pharmacodynamics can be observed in Table 1.

\begin{tabular}{|c|c|}
\hline Properties & ce on pharmacokinetics and pharmacodynamics \\
\hline Composition & $\begin{array}{l}\text { - It is easier for silica NBMs the reaching of lungs instead of polymeric } \\
\text { NBMs }\end{array}$ \\
\hline Size & $\begin{array}{l}\text { - NBMs of about } 100 \mathrm{~nm} \text { have longer circulation times } \\
\text { - NBMs }<6 \mathrm{~nm} \text { are eliminated through renal filtration } \\
\text { - NBMs from } 10 \text { to } 12 \mathrm{~nm} \text { show high permeation and low accumulation in } \\
\text { tissue/organs } \\
\text { - NBMs }>200 \mathrm{~nm} \text { are recognized by the mononuclearphagocyte system } \\
\text { MPS. Furthermore, they are retained by splenic filtration }\end{array}$ \\
\hline Shape & $\begin{array}{l}\text { - A deviation from the spherical shape will increase the circulation time } \\
\text { - Rod-shaped particles are easily taken up by cells }\end{array}$ \\
\hline Surface charge & $\begin{array}{l}\text { - Positively charged NBMs are able to form aggregates in the presence of } \\
\text { negative charged proteins. Aggregates may cause embolism in the lung } \\
\text { capillaries, while protein corona formation may lead to clearance by MPS } \\
\text { - Negatively and neutral charged NBMs show longer circulation half-lives }\end{array}$ \\
\hline Surface Chemistry & $\begin{array}{l}\text { - The surface modification of a NBM surface with non-ionic polymers is } \\
\text { able to decrease the risk of opsonization, increase blood circulation time, } \\
\text { reduce interaction between NBMs and the target cell } \\
\text { - The surface modification using targeting moieties able to bind selectively } \\
\text { to cellular receptors is able to increase specific cellular interactions }\end{array}$ \\
\hline
\end{tabular}



pharmacodynamics.

Polymeric NBMs might be eliminated from the body through degradation or they can be expelled by liver, kidneys or colon. Such elimination is affected by several physicochemical properties of the NBMs. A material is termed biodegradable if it is able to be decomposed or mineralized into end products by biological activity as a part of its degradation process. The most common degradation mechanisms are hydrolysis, oxidation and enzymatic reactions.

The polymers that are susceptible to hydrolysis are the one with hydrolysable backbones, such as polyesters or polyanhydrides. Other polymers, such as PEG and polyethylene are more suitable to degradation through oxidation reactions, since they facilitate radicals' formation. The mechanism beyond an enzymatic degradation is a hydrolysis catalyzed by enzymes (hydrolases and lipases). Different natural polymers undergo this type of degradation, but various techniques exist to reduce its rate through, for instance, acetylation or PEGylation. Biodegradable polymers show advantages with respect to the non-biodegradable ones, since usually the products of degradation reactions are non-toxic and can be eliminated from the body thanks to the natural metabolic ways. Sometimes, products of degradation may also be able to generate an inflammatory response: this should be taken into account during the biocompatibility evaluation of any biodegradable polymer. The degradation rate play an important role in the biocompatibility, for instance a fast-degradable material could produce such a large amount of degradation products to overwhelm the tissue's removal mechanism. Luckily, some material properties can be tuned in order to achieve the desired degradation rate for each application. On the contrary, if the material is not degradable, it has to be eliminated from the body, but this is possible only with NBMs whose dimensions are lower than $6 \mathrm{~nm}$. Nonetheless, small particles can accumulate in tissue and cause toxicity if highly positive. Furthermore, nanoparticles bigger than $6 \mathrm{~nm}$ can also be taken up by phagocyte and, if not degradable, they will remain in those cells and be sequestered in the spleen and liver for up to 6 months. 
As an alternative, NBMs are believed to be excreted from the hepatocytes: depending on their composition NBMs can be excreted as a bile and pass eventually in the small intestine. All the exposure to a polymeric nanoparticle should thus be taken into consideration. An exposure assessment should include an estimation on the dosage, duration of the exposure and the predicted administration and/or exposure route. These parameters are well defined for patient exposure, since they should be defined in order to reach the desired therapeutic efficacy. The problem is related to the absence of methods for the detection and quantification of unintentionally absorbed NBMs. The in vitro simulation of realistic human exposure is a challenging process also for nanomedicine. One of the problems is related to the transfer of human doses to in vitro settings. Nevertheless, it is difficult to build up complex in vitro systems able to perfectly mimic the physiological complexity of the human body. After all, most in vitro studies use higher concentrations of polymeric NBMs with respect to those that should be used in vivo, thus limiting the similarity with a real exposure.

\subsection{HAZARD}

The toxicological effect of NBMs is a result from their small particle size and greater particle surface area with respect to their bulk materials, since these two properties are responsible of an increase in their reactivity. Also others properties influence the toxicity, such as the chemical composition or the surface charge. The principal mechanisms through which the polymeric NBMs affect biological systems are cellular uptake, oxidative stress, cellular membrane damage, inflammation and DNA damage. These processes, alone or staking their effects, could bring a significant impact on human health. Unfortunately, some results of toxicology tests are ambiguous, probably as a consequence of the large variety of methodologies used and the difference among the physicochemical properties of every NBM. Most studies are also performed with drug loaded NBMs and without an evaluation of the unloaded polymer. The problems with this type of results is that it is not possible to define if some effects are imputable to the drug, the NBM or both. 
Furthermore, also the testing for contaminants is usually lacking from the reports. The gaps in the data available have made it difficult to identify trends in the toxicity of most of the polymeric NBMs studied. It is important to highlight that this absence play a major role in preventing a safer material design based on literature. Therefore, experiments are needed in order to fill those gaps. It can be thus observed that this is a Safe-by-Design iterative procedure: the experimental data that have been characterized can then be used for refining material design used in (Q)SAR models for example. A polymeric NBM hazard assessment should include proper in vitro and, if necessary, in vivo assays. Such assays should include the following endpoints:

- Immunotoxicity studies, such as oxidative stress and inflammation,

- Genotoxicity,

- Toxicity on reproduction,

- Biocompatibility and haemocompatibility,

- Acute, repeated or chronic toxicity studies.

It would be better to use standardized methodologies for NBM and with suitable controls in order to minimize the different in the results from different research groups. Up to now, no real guidelines have been released on polymeric NBMs by competent authorities. Nevertheless, some information can be found on reflection papers on coated nanomedicine products and block copolymer-micelle medicinal products released by the EMA, since they anticipate the parameters that should be included in applications for MA. For marketing authorization, more endpoints are needed than those proposed for human health risk assessment. In this view, the ICH Safety Guidelines should be considered for nanopharmaceuticals. As far as medical devices are concerned, it is also important to consider ISO 10993, which contains several standards for biological evaluation of these devices. 
The OECD Working Party on Manufactured Nanomaterials (WPMN) aims to promote a cooperation among different nations on the safety of nanomaterials with respect to human health and environment, including safety testing and risk assessment. Different reports have been published in the recent years by the WPMN and are all collected in the OECD Series on the Safety of Manufactured Nanomaterials. Toxicity testing bring different challenges, such as:

- Difficulty in the simulation of realistic exposure scenario in vitro.

- Nanoscale properties that can interfere with reactants and detection methods during in vitro assays, such as protein corona formation, dissolution and/or generation of new nano-sized particles, when in contact with biological matrices.

- Interference of polymeric NBMs with endotoxin quantification essays.

- Absence of positive and negative controls for nanoscale materials in toxicity studies,

- Strong dependence of the final results from the chosen cell line, incubation time, cell culture media and cell culture supplementation.

A general decision tree covering every topic discussed above is illustrated in Figure 5, used as a summary. Here also other parts are present, such as the environmental risks and the storage \& transport, that have not been covered in the discussion. The green arrows represent the flow of polymeric NBMs from their design up to their storage and transport, while red one are feedback loop to be used whenever the NBM is unsafe. 


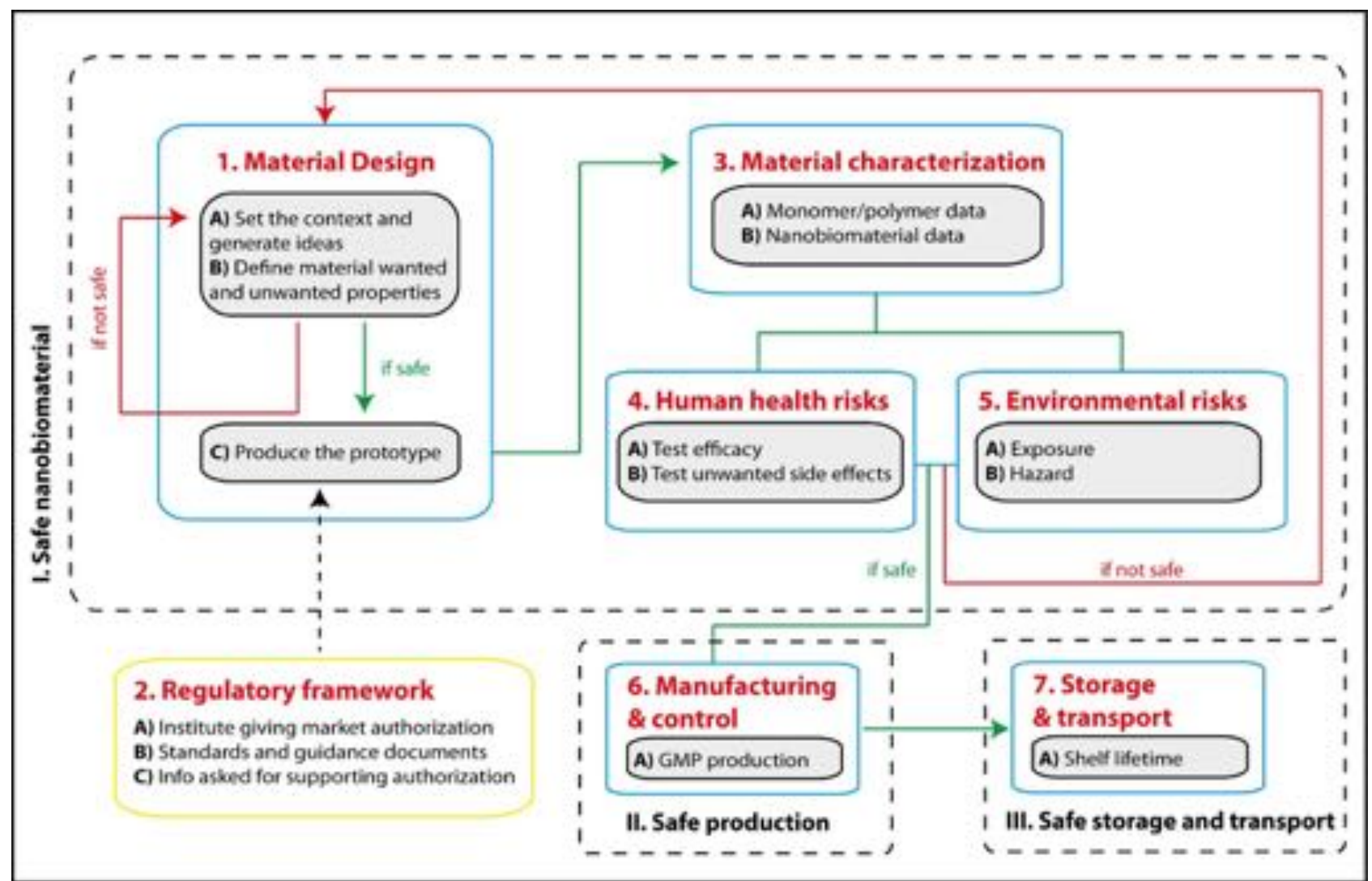

Figure 5 - GoNanobioMat framework. Green arrows correspond to the flow of polymeric nanobiomaterials as drug delivery systems from design to storage and transport, red arrows are feedback loops used whenever the nanobiomaterial product is unsafe, inefficient or has unwanted side effects. 


\title{
Chapter 13
}

Regulatory Perspectives on Medical Nanotechnologies

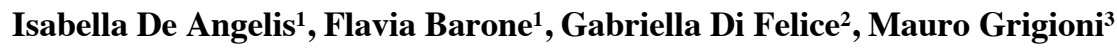

${ }^{1)}$ Department of Environment and Health ${ }^{2)}$ National Center for Drug Research and Evaluation, ${ }^{3)}$ National

Center for Innovative Technologies in Public Health, Italian National Institute of Health, Rome, Italy

\section{[NON PRINT ITEMS]}

\begin{abstract}
Manufactured Nanomaterials (MNMs) are particularly attractive for innovative industrial sectors and for nanomedicine application. They are already present on the market both in consumer products and bi

omedical applications and are expected to grow further in the near future. Conversely, several concerns about MNMs potential effects on human health and environment as well as potential risks due to the exposure to MNMs are still open. For this reason, European regulatory bodies are particularly active to develop specific regulatory framework for MNMs marketing authorization. To date, the European Union (EU) covers MNMs, and, in particular, the potential risks associated with them, by the existing legislation for chemical substances, pharmaceutical products, and medical devices, even if nanomaterials are not always explicitly mentioned. This chapter presents a brief overview of some horizontal and sector-specific legislations concerning the main application sectors of MNMs outlining possible future regulatory scenarios.
\end{abstract}

Key Words: Manufactured Nanomaterials, Nanomedicine, Nanodrugs, Medical Devices, EU legislation, Nanomaterials Regulatory framework

\section{[Chapter Starts Here]}

\section{Introduction}


Nanotechnology has been included by European Commission (EC) among the six Key Enabling Technologies (KETs)

(EC, 2009a), namely technologies with great potential for societal and economic improvement and sustainable development. During the last ten years, nanotechnology has emerged in a broad area of industries and applications. In its Opinion 002/05, the Scientific Committee on Emerging and Newly Identified Health Risks (SCENIHR) defined Nanotechnology as "the design, characterization, production and application of structures, devices and systems by controlling shape and size at the nanoscale", and Nanoscience as "the study of phenomena and manipulation of materials at atomic, molecular and macromolecular scales, where properties differ significantly from those at a larger scale" (SCHENIR, 2006).

Manufactured nanomaterials (MNMs) are chemical compounds and/or materials in which the very low dimensions (in the range of nanometer) determine peculiar and novel physico-chemical properties in respect to the same material not in nanoform. These properties are mainly due to the increased surface area-volume ratio that involves a greater percentage of surface atoms than the internal ones, which in turn determines an increased reactivity, greater conductivity and electrical resistance, and potentially greater biological activity. These properties make them particularly attractive for innovative industrial sectors spanning from public health, energy, environment, transport, and communication and, consequently, for their use in different fields of application as chemical, pharmaceutical, agro-food and biomedical.

\subsection{EU Definition of Nanomaterials}

EC adopted in 2011 a Recommendation on the definition of nanomaterial (2011/696/EU). A material in nanoscale (or nanomaterial) is currently defined as " a natural, incidental or manufactured material containing particles, in an unbound state or as an aggregate or as an agglomerate and where, for $50 \%$ or more of the particles in the number size distribution, one or more external dimensions is in the size range $1 \mathrm{~nm}-100 \mathrm{~nm}$ " (EC, 2011). In this Recommendation "particle" means a minute piece of matter with defined physical boundaries, "agglomerate" means a collection of weakly bound particles or aggregates where the resulting external surface area is similar to the sum of the surface areas of the individual components, "aggregate" means a particle comprising of strongly bound or fused particles. Natural nanomaterials are widespread in the ecosystem and derive from biological and geological processes, such as natural combustion processes or volcanic eruptions. Incidental nanomaterials are unintentionally produced and derive e.g. from vehicular traffic, diesel engines, industrial incinerators, during welding operations and laser printing processes of copiers. MNMs are intentionally produced for scientific and industrial purposes and have a well-defined chemical composition.

The purpose of the EC Recommendation is to ensure consistency across different regulatory areas. European Member States (EMS), Union Agencies and economic operators are invited to comply with this definition, for example in the 
adoption and implementation of legislation and policy and research programmes concerning products of nanotechnologies.

\subsection{General concepts of nanosafety}

Despite the exciting possibilities of MNMs applications these materials may raise several concerns about their potential effects on human health and environment and many scientific questions on potential risks of exposure to MNMs are still open (Gottardo et al., 2017). The hazard profile is extremely variable between different nanomaterials. Up to now, a multitude of both in vivo and in vitro studies produced conflicting toxicological results and a clear association between nano-dimension and hazard has not been proven. In 2009, SCENIHR stated that "The health and environmental hazards were demonstrated for a variety of manufactured nanomaterials. The identified hazards indicate potential toxic effects of nanomaterials for man and environment. However, it should be noted that not all nanomaterials induce toxic effects... In this respect, nanomaterials are similar to normal substances in that some may be toxic and some may not. As there is not yet a generally applicable paradigm for nanomaterial hazard identification, a case by case approach for the risk assessment of nanomaterials is recommended." (SCENIHR, 2009). In their joint report on "Impact of Engineered Nanomaterials on Health: Considerations for Benefit-Risk Assessment", EASAC (European Academies Science Advisory Council) and EC Joint Research Centre concluded "...there is only a limited amount of scientific evidence to suggest that nanomaterials present a risk for human health" (EASAC-JRC, 2011).

To date, risk evaluation of MNMs is carried out in the framework of the current risk assessment paradigm applied to conventional bulk materials. The crucial question remains the possibility to apply the existing Test Guidelines (TGs) to predict specific nano-effects and, eventually, how they should be adapted for this purpose. Another critical point is the lack of consolidated scientific knowledge of MNMs properties based on standardized methodological approaches, in particular for physico-chemical characterization (OECD, 2016).

Due to the benefits derived from nanotechnologies, and since MNMs are already present on the market, and are expected to further grow very fast in the near future, European regulatory bodies (European Scientific Committees and Agencies) as well as United States and Asian developed countries, are actively proceeding to develop sector regulations and tools (as TGs, best practices, standard methodologies) for nanosafety assessment. In addition, a robust international cooperation involving organization as OECD (Organization for Economic Co-operation and Development) and ISO (International Organization for Standardization) is taking place on this topic.

From the European perspective, MNMs safety has a very high level of concern and a lot of efforts and financial resources have been put in place. Since the fifth EU Framework Programme for Research and Technological Development (1998-2002), EC has started funding projects specifically addressing nanosafety with a constant budget 
increase. Moreover, all these EU projects were connected through the NanoSafety Cluster

(https://www.nanosafetycluster.eu/) to maximise synergies, improving the coherence of nanotoxicological studies, and harmonizing protocols and methodologies. NanoSafety Cluster also acts as spokesperson in discussions on MNMs risk/benefit balance with industrial stakeholders and public opinion.

Another important point faced by EU is the establishment of a harmonized database on MNMs currently on the market. There are several publicly available information mainly derived from REACH (Registration, Evaluation, Authorization and Restriction of Chemicals) registrations, Cosmetics Regulation, EMS national inventories, such as French and Belgian inventories. Moreover, different types of database are now available, as eNanoMapper (https://search.data.enanomapper.net/) and NanoData (https://euon.echa.europa.eu/it/nanodata). The first, funded by EU programme on Research and Innovation, provides computational infrastructure for the management of MNMs toxicological data. It is one of the main sources of currently available data on the toxicological properties of MNMs. The latter is focused on development of MNMs and nanotechnology in EU in different fields of application (health, energy, manufacturing, information and communication technology). All these sources of information are linked to ECHA’s (European Chemical Agency) chemicals database.

At the international level, OECD launched in 2006 the Working Party on Manufactured Nanomaterials (WPMN) with the purpose to develop a globally harmonised science-based approach to the management of MNMs. During more than ten years of activity, WPMN performed a considerable amount of work declassifying many technical reports. Moreover, as reported in its Council Recommendation (OECD, 2013), OECD highlights the need to adapt the existing TGs to the specific characteristics of MNMs addressing nano-specific issues. Consequently, OECD recently started with the accommodation and/or the development of new TGs and Guidance Documents (GD) specific for MNMs, with the financial support of EMS.

\section{Regulatory drivers for MNMs}

In the EC nanomaterials are covered by the same rigorous regulatory framework that ensures the safe use of all chemicals and mixtures, i.e. the Registration, Evaluation, Authorization and Restriction of Chemicals (REACH) (EC, 2006) and the Classification, Labelling and Packaging (CLP) regulations (EC, 2008). This means that the hazardous properties of nanoforms of substances have to be assessed in order to ensure their safe use in all the products placed on the market. Moreover, some sector-specific legislations such as food, biocides and cosmetics, contain specific provisions for MNMs. In general, the current European regulatory framework supervises the presence of MNMs in commercial products even if it is not fully harmonized.

Table 13.1, reports a summary of the different EU legislations for MNMs. 
[insert table 13.1 here]

\subsection{Regulatory aspects: non-medical products}

To be legally manufactured or imported in the EC all substances that fall within the scope of REACH have to be registered. According to the volume placed on the market, manufactures or importers must submit information on both human health and environmental effects, and an estimate of exposure throughout the life cycle. MNMs fall under REACH definition of "substance", but no specific requirements for nanoform are reported in the regulation test. REACH applies the EU definition of MNMs and ECHA published a specific document to define the term "nanoform" (ECHA, 2017). Moreover, MNMs that fulfill the criteria for classification as hazardous materials must be classified and labelled accordingly to CLP Regulation.

In December 2018, the Regulation 2018/1881/EU amended REACH regulation through the revision of the REACH Annexes introducing nano-specific clarifications and new provisions in the chemical safety assessment (Annex I), registration information requirements (Annex III and VI-XI) and downstream user obligations (Annex XII) (EC, 2018). It has entered into force on 1 January 2020. New requirements make explicit that nanoforms of substances need to be covered by the registration dossiers and more detailed information on them have to be reported. ECHA recently published a specific guidance to address the preparation of registration dossiers for MNMs (ECHA, 2019).

EU sector-specific regulations relevant for MNMs are:

\section{Cosmetics}

The EC Regulation 1223/2009 (EC, 2009b) introduced specific provisions for use of MNMs in cosmetic products. It includes a definition of MNMs and requires for premarket notification, safety evaluation and labelling of MNMs used in cosmetics. According to Cosmetic Regulation a MNMs is "An insoluble or biopersistent and intentionally manufactured material with one or more external dimensions, or an internal structure, on the scale from 1 to 100 nm". This definition diverges from EC 2011/696 recommendation covering only insoluble/biopersistent MNMs (e.g., metals, metal oxides, carbon materials), while persistent MNMs in biological systems as liposomes or oil/water emulsion are excluded. Nevertheless, in a recent opinion, the SCCS (Scientific Committee on Consumer Safety) recommends to the applicants to take into account in the safety assessment of MNMs the EU Recommendation (SCCS, 2019). Finally, the presence of nanoform of ingredients must be labelled with the word "nano" in brackets following the name of the substance. EC is also responsible for publishing a catalogue of all MNMs used in cosmetic products on the EU market (https://ec .europa.eu/docsroom/documents/38164).

\section{Biocidal}


The Biocidal Products (BPs) Regulation, (EU, 2012), has specific rules for nanomaterials. Provisions apply to products and substances that meet the 2011/696/EU Recommendation on nanomaterial definition. A separate dossier with all data requirements and a dedicate risk assessment must usually be prepared for nanoforms of active and non-active substances used in BPs. Any MNM presents in a BP have to be clearly reported in the label, followed by the word "nano" in brackets. Finally, products containing nanomaterials are excluded from the simplified authorisation procedure. Some MNMs are already approved for biocidal use as, for example, silicon dioxide.

\section{Food and Feed}

Due to the increased use of MNMs in the food and feed chain, it is of great importance to determine whether they raise any potential health or environmental concerns. The European Food Safety Authority (EFSA) is the responsible in the EU for the risk assessment of MNMs used in food and feed and in food contact materials as well. Different EU regulations cover the use of MNMs in food sector. First of all, the Novel Foods Regulation (EU, 2015) addresses to MNMs including requirements for placing them on the EU market. In general, MNMs have to be approved and authorized by EFSA scientific panels before being used in food/feed products. Drivers for novel food approval are: i) not risk to public health, ii) not nutritionally disadvantageous when replacing a similar food, iii) not misleading to the consumer. Nanomaterials definition in the Novel Foods Regulation is quite different from the EC Recommendation; for example, it does not fix a threshold of particles in the number size distribution to define a material as a nanomaterial. Provisions for MNMs are also present in different pieces of EU legislation on food and feed products, for example:

- Food Additives Regulation (EC, 2008) reports a positive list of approved additives, enzymes and flavorings.

Substances already included in the list need to be re-evaluated if there are changes in the production process (e.g. by use of nanotechnologies) and/or in the starting materials (e.g. different particle size)

- Plastic Food Contact Materials Regulation (EU, 2011b) deals with the potential release of chemicals from food contact materials avoiding the use of harmful chemicals in this material. Nanoform of substances can only be used in plastic materials if they are explicitly authorised or are included in a positive list of admitted substances established by EC as, for example, carbon black and titanium nitride nanoparticles

- Food Information to Consumers Regulation (EU, 2011a), regulates the labelling of food ingredients, including MNMs. These last have to be clearly indicated in the list of ingredients with the ingredient name followed by the word "nano" in brackets.

\subsection{Regulatory aspects: pharmaceutical products}

In the last decades, biomedical applications based on nano-sized materials have been proposed to develop new drug formulations with improved stability, bioavailability, favorable biodistribution profiles and the capability of targeting 
specific cell populations. Different nanotechnology tools are used to improve drug solubility (micelles and nanocrystals), to guide drugs to the intended cell or tissue target (targeting nanocarriers), to control the drug's release (nanoparticles and liposomes), to enhance the transport across biological barriers (micelles and nanoparticles) (Hafner et al., 2014).

The use of nanotechnology has been recognized in the EU as a Key Enabling Technology, capable of providing new and innovative medical solution to address unmet medical needs (Pita et al., 2016). The application of nanotechnology for medical purposes has been termed nanomedicine and is defined as the use of nanomaterials for diagnosis, monitoring, control, prevention and treatment of diseases (Tinkle et al., 2014). Nanodrugs are nanostructured or nano-scale materials, engineered to obtain particular medical effects based on their structure. Their chemical composition and physical properties, including size/hydrodynamic radius, morphology, surface chemistry, solubility, and charge, can be engineered to make them suitable for specific biomedical applications. On the other hand, the same physico-chemical properties primarily affect the biodistribution of nano-sized materials after entering the body through different routes, the cellular uptake mechanisms, and their potential toxicity (Nystrom and Fadeel, 2012).

To define a product as a nanodrug according to the size range, it is important to note that the size limit of $100 \mathrm{~nm}$ (as reported in the EU 2011/696 Recommendation) is not strictly applied for pharmaceutical products. It is widely recognized that other factors should be taken in account to also include all the "structures" with size less than $1000 \mathrm{~nm}$ that are designed to have specific physico-chemical, biologic and physiologic properties (Hernan Perez de la Ossa, 2014). Most of the investigational and approved nanomedicine products contain nanocomponents with a mean size of 0-300 $\mathrm{nm}$ and nanocrystal dispersions that resulted in sizes up to $2000 \mathrm{~nm}$.

Nanodrugs are characterized by complex mechanisms of action combining mechanical, chemical, pharmacological, immunological characteristics, or diagnostic as well as therapeutic functions. In some cases, it is difficult to classify these products as drugs or medical devices to apply the correct regulatory procedure; the prevalent mechanism of action can be regarded as a major principle.

The introduction of nanotechnologies in drug development mainly addressed the therapy of cancer and infectious diseases, with the aim to encounter unmet medical needs in these fields, by enhancing efficacy while reducing potential side effects.

A long and complex process underlies the development of a nano-scale product intended for medical application; in particular, the basic research and the preclinical studies may last several years and the whole process can go over 20 years (Etheridge et al., 2013). 
From the regulatory point of view, European and worldwide agencies have recognized the peculiar critical aspects of the nanodrug development process, specifically related to their nano-scale nature. The challenge is to ensure the proper evaluation of the quality, safety, and effectiveness of nanodrugs undergoing clinical development and market authorization (Soares et al., 2018). Quality aspects include, among others, the product characterization, the control of the manufacturing process (scaling-up and reproducibility are crucial points), the stability of the intermediate and final drug product. Regarding efficacy and pre-clinical safety, it is crucial to characterize the functional interactions at the nanodrug surface, the pharmacodynamics/pharmacology, the pharmacokinetics (biodistribution, metabolic fate, with particular attention to persistence in cells, tissues, interstitial spaces), the pre-clinical safety (short and long term), the interaction with the host immune system. It is important to underline that the nanodrug safety assessment often does not overlap with classical toxicology evaluation, since primary or secondary pharmacodynamics effects can interfere, as well as the effects of dose and administration route/frequency, and the clinical status of patients' population. Last, but not for relevance, is the issue of environmental impact in terms of use and disposal of nanodrugs. For regulatory purposes, nanodrugs are under the framework set by European Medicines Agency (EMA) (Ehmann et al., 2013). Since 2006, EMA established a cross-agency Nanomedicine Expert Group, subsequently expanded in 2009 through the launch of a joint initiative by the regulatory agencies of the EU (EMA), USA (FDA), Japan (Ministry of Health, Labour and Welfare) and Canada (Health Canada): the International Regulatory Subgroup on Nanomedicines. The EMA Reflection Paper on Nanotechnology-based Medicinal Products for Human Use, issued in 2006, provided early information about the European Commission, the EMA experience and perspectives and an official definition of nanomedicine (EMA, 2006). Then, in 2011, the EMA Committee for Human Medicinal Products (CHMP) established the Multidisciplinary Expert Group on Nanomedicines (Figure 13.1). Members from scientific community and regulatory network gathered to collect multidisciplinary scientific information about nanodrugs in order to develop new ad hoc guidelines or review the existing ones. To provide guide and support to applicants in order to obtain approval through centralized procedure at the European level, EMA has elaborated several Reflection Papers specific for selected nanodrug categories. These documents are mainly focused on the pharmaceutical development, nonclinical and early clinical studies needed for marketing authorization of micellar systems (EMA, 2012), surface-coated nanoparticles (EMA, 2013a), liposomal products (EMA, 2013b), block copolimer micelles (EMA, 2014), nano-colloidal iron-based preparations (EMA, 2015). Besides these classes of products, EMA is willing to provide Scientific Advice on a case-bycase basis regarding preauthorization studies for assessing the quality, safety, and efficacy profile of new nanodrugs, through the CHMP Expert Group. All these documents and the information about EMA initiatives and advice are available in the EMA website [insert figure 13.1 here]. 
Up to now, the European experience with nanomedicines records the approval of several categories of the so-called “first generation nanodrugs" by centralized or mutual recognition procedures, under the current regulatory framework (Ehmann et al., 2013; Musazzi et al., 2017). However, several specific safety issues still remain critical, including: 1) the set-up and validation of standardized in vitro assays, with suitable reference materials; 2) the development of $e x$ vivo/in vivo models relevant for the administration route; 3 ) the generation of in silico approaches which are predictive for biological and toxicological responses; 4) the suitability of in vivo pharmacokinetic studies (Kaur et al., 2014). Finally, the interactions of nanotechnology-based drugs with the major biological systems deserve particular attention from coordinated contributions of multidisciplinary competences.

New challenges for the regulatory activity are approaching, from future or already present perspectives. The advent of "nanosimilars" (similar nanodrugs arising as first generation products come off-patent) highlights the need for comparability studies in relation to the reference medicine to support the similar nature in terms of bioequivalence, quality, safety and efficacy, before marketing authorization (Ehmann et al., 2013; Soares et al., 2018). This exercise is particularly challenging for complex biological and biotechnological drugs, such nanotechnology-based medicinal products. In parallel, "next-generation" nanodrugs that are been developing based on the recent advances in nanoscience include increasingly complex structures, often combining different functions. The correct and comprehensive evaluation needed before the approval of first-in-man studies warrants specific regulatory consideration.

\subsection{Regulatory aspects: Medical Devices}

The field of Medical Devices (MDs) comprises many different technologies, promoting more than 500.000 class of products, from sticking plasters to diagnostic apparatuses. Currently nanoparticles are used as devices per se or are used within the design of new MDs, to improve both diagnostic and therapeutically capability, e.g., to prevent sepsis, just to name one of the many possible functions. Since two decades nanomaterials are present in almost any industrial sector focusing at first on free, non-degradable and insoluble nanoparticles found in medical applications, food, consumer products and environment. In the case of MDs, the new Regulation (EU) 2017/745 (EU, 2017) is provided with an appropriate definition (also in accordance with the EU Recommendation 2011/696), allowing to investigate the risk of MDs making use of nanostructures and to classify them in the appropriate risk class, indicating moreover the appropriate assessment route.

Unfortunately, some uncertainties regarding the safety of MNMs still exist, thus different organizations and committees have been addressing the identification of risks and the related tests to assess them (SCENIHR, 2015). Moreover, different harmonised definitions (ISO, MDs Regulation (MDR)) of nanoscale have been proposed, with a certain delay after the required scientific debate, but a universally agreed definition in still missing. Starting from that, best practices 
were developed in order to facilitate harmonization of assessment practices, especially in the biomedical field. In this respect MDR approached this field with the appropriate classification rule based on risk for patients at first, then for operator and environment (EU 2017/745). The highest risk class (III) was set for MD produced with MNMs, according to the MDR's Rule 19, whenever the devices present a high or medium potential for internal exposure. However, this classification of MDs was substantially driven by the uncertainties with respect to the known risk with time. More research with specific relevance for regulatory provisions and questions is still needed, in particular regarding the implementation of the definition of MNMs, the enforcement of product labeling, the development of methods for safety testing and risk assessment, and a better availability of quality data on MNMs for regulatory purposes.

In the field of harmonised standards (international or EU Guidelines) at disposal for biocompatibility and toxicity testing, the ISO 10993 series have proven themselves to be appropriate for any kind of materials but those in nanoform (ISO, 2017), especially for certain class of MNMs (e.g. some metal oxides), interfering with several techniques indicated in the ISO 10993 standard series (Lupu and Popescu, 2013). Thus current risk assessment methods with regard to the biological evaluation of MDs are applicable in part also to MNM-containing MDs, even though further research on particular aspects of risk assessment is still required for certain class of MNMs.

In the meantime, the types of function for MD making use of MNMs are seen to be growing up steadily.

From the first generation, new products have been developed which bring together various fields of application: 1) nanostructures with passive functions often applied in types of products which already exist (e.g. surface cover to improve biocompatibile contact with biological tissue); 2) nanostructures which can exhibit a change in properties (e.g. nanoparticles targeting pharmaceuticals to tumour cells, and releasing the pharmaceutical in the tumour under the influence of a radiation source); 3) networks of nanosystems and robotics on a nanoscale; 4) molecular nanosystems can be designed for advanced genetic therapies and self-assembling structures on a nanoscale.

Potential risks were identified by the scientific community when applying these technologies directly to consumers, workers and the environment. As well as MNMs per se, in the medical field, any product added or made with nanomaterial or more complex nanostructure could be released at a certain stage in the life cycle of that particular product; in the latter case it is mandatory to refer to Reach regulatory framework for the nanomaterial per se, when particles might be released, then to proceed with the provisions of the MDR.

To date, the recent MD Regulation provides three different risk classes for MDs making use of nanostructures, assigning class IIb (one step lower than the maximum risk class, III) to the MDs that present a low potential for internal exposure, and class IIa (with an even lower risk) to the MDs that present a negligible potential for internal exposure, the potential level depending on the technology used to limit exposure. 
That risk level depends on the possibility that nanoparticles (also as aggregates or agglomerates) could meet cellular membranes, with their possible internalisation within the cell, determining cell damage of different gravity. These risks are, however, more difficult to be determined than those of chemical substances not in a nanoform, and are, to some extent, still largely unknown. MNMs by themselves can be present as powders or colloid dispersions, but also can be present in MDs while incorporated in a matrix, as nanostructured material or as surface structures on materials and/or MDs e.g. to avoid clotting and retard thrombus formation when in contact with blood. Thus morphological structures created e.g. on the surface of aMD, can also have sizes in the nanoscale, with possible effects on the biological response to the device due to release of nanostructures during the life cycle of aMD, with possible adverse effects following the preparation, use, wear or degradation of MDs. For the biological evaluation of MD, knowledge on the potential generation and/or release of nano-objects from such materials is essential.

In general, MNMs themselves need to be evaluated instead of extracts as usually done when testing biomaterials or MDs. Also the use of harmonised guideline such as EN ISO 10993 series on Biocompatiblity for Medical Device (ISO, 2017) was demonstrated to be not completely appropriate since the MNMs could interfere with the methodology, resulting in not accurate results (see the case of metal oxides for which several tests are reported in the normative). Research in this field is continuously promoting comparison of test methods and results on MNM.

The procedures described in the ISO 10993 series for the biological evaluation of MDs can be used for the biological evaluation of those MDs that contain nano-objects that are not released from such a device, as they are an integrated part of the device. However, when the release of the nano-objects is possible, a safety evaluation should also be performed on released nano-objects. In addition to evaluating a MD, MNMs components or constituents can also be separately evaluated.

Thus, MNMs pose specific challenges when applying test systems commonly used for MD evaluation and when interpreting test results.

Typically, the assessment of the potential risk from the use of MNMs in MDs is mainly associated with the possibility for release of free nanoparticles from the device and the duration of exposure. Moreover, the assessment of comparable devices not incorporating nanomaterials could be useful in the decision about the acceptability of the risk.

\section{Conclusions}

The rapid evolution of MNMs and nanoproducts has created the need for a similarly rapid advancement in scientific knowledge and its translation into regulations, Guidances and OECD TGs to address the safety of MNMs. Consequently, there is a clear need for an inclusive and science-based risk governance process (Van Teunenbroek, 2017). In this process the principles of governance are applied to the identification, assessment, management, evaluation and communication 
of risks (https://irgc.org/) (Figure 13.2). Involvement of all the different actors is a crucial step of this process, taking into account their specific rules.

From a risk governance point of view, it has become a real challenge to accommodate nanotechnology correctly and uniformly across all involved regulatory domains. In 2018, EC funded three projects that will deal with this issue (EU H2020 Risk Governance of nanotechnology - Foundations for tomorrow's industry). The final goal will be to establish an international Nano Risk Governance Council (NRGC) that will engage a broad variety of stakeholders across all relevant nano-disciplines (chemical, biocides, food and feed, pharma and medical devices and materials development). [insert figure 13.2 here]

For nanopharmaceutical application, the global opinion, shared by EMA and other regulatory agencies worldwide, is that the current regulatory framework is suitable to evaluate and authorize nanodrugs. The current regulatory framework is also considered robust to approach the certification of MDs, thanks to the basic guideline ISO EN 14971 (Risk assessment and management methodology) cited into the EC Directives and MDR. However, a scientific gap still persists among current knowledge and (emerging) nanoproducts (innovative nanostructured products, MDs, and nanodrugs). To overcome this gap, specific competences should be implemented by the contribution of multidisciplinary experts. The potential solutions proposed to improve the evaluation process include: 1) the development and validation of new or amended methods to implement existing TGs; 2) the early evaluation of emerging risks and potential critical aspects; 3 ) the promotion of scientific interaction among academy, regulatory agencies and industry to foster collaboration and harmonization at the international level.

Multidisciplinarity, sharing of technical and scientific information, together with a strong coordination of the methodological improvements, are the weapons useful to guarantee citizens (patients and workers), environment, manufacturers and public institutions, promoting a new global approach for the safety and efficacy of any innovation.

$* * *$ Insert Figure $\mathrm{x} . \mathrm{x} * * *$

Caption:

Figure 13.1. EMA initiatives for the regulatory evaluation of nanotechnology-based pharmaceutical products

Figure 13.2. Principles of Risk Governance

Credit:

\section{[Chapter References]}


1. Ehmann, F., Sakai-Kato, K., Duncan, R., Hernan Perez de la Ossa, D., Pita, R., Vidal, J.-M., et al., 2013. Next-generation nanomedicines and nanosimilars: EU regulators' initiatives relating to the development and evaluation of nanomedicines. Nanomedicine-UK 8 (5), 849 856.

2. Etheridge, M.L., Campbell, S.A., Erdman, A.G., Haynes, C.L, Wolf, S.M., McCullough, J., 2013. The big picture on nanomedicine: the state of investigational and approved nanomedicine products. Nanomedicine: NBM 9 (1), 1-14.

3. European Academies Science Advisory Council (EASAC), 2011. Impact of Engineered Nanomaterials on Health: Considerations for Benefit-Risk Assessment. Joint EASAC-JRC Report.

https://publications.jrc.ec.europa.eu/repository/bitstream/JRC64792/impact\%20of\%20engin eered\%20nanomaterials\%20on\%20health.pdf (accessed 10.02.2020.).

4. European Chemical Agency (ECHA), 2017. How to prepare registration dossiers that cover nanoforms: best practices. Helsinki: ECHA, ECHA-17-G-13-EN. https://echa.europa.eu/documents/10162/13655/how to register nano en.pdf (accessed 10.02.2020.).

5. European Chemical Agency (ECHA), 2019. Appendix for nanoforms applicable to the Guidance on Registration and Substance Identification. Helsinki: ECHA-19-H-14-EN. https://echa.europa.eu/documents/10162/23047722/appendix nanoforms msc rac forum e n.pdf/a68660cd-4cf2-7437-cf0b-56e628a48d76 (accessed 10.02.2020.).

6. European Commission (EC), 2006. Regulation (EC) No 1907/2006 of the European Parliament and of the Council of 18 December 2006 on the Registration, Evaluation, Authorisation and Restriction of Chemicals (REACH). Official Journal of the European Union No. L396, 30.12.2006.

7. European Commission (EC) 2008a. Regulation (EC) No 1272/2008 of the European Parliament and of the Council of 16 December 2008 on classification, labelling and 
packaging of sub substances and mixtures (CLP). Official Journal of the European Union No. L353, 31.12.2008.

8. European Commission (EC), 2008b. Regulation (EC) No 1333/2008 of the European Parliament and of the Council of 16 December 2008 on food additives. Official Journal of the European Union No. L354, 31.12.2008.

9. European Commission (EC), 2009a. Preparing for our future: Developing a common strategy for key enabling technologies in the EU. Communication from the Commission to the European Parliament, the Council, the European Economic and Social Committee and the Committee of the Regions \{SEC(2009)1257\}. https://eur-lex.europa.eu/legalcontent/EN/TXT/PDF/?uri=CELEX:52009DC0512\&from=EN (accessed 10.02.2020.).

10. European Commission (EC), 2009b. Regulation (EC) No 1223/2009 of the European Parliament and of the Council of 30 November 2009 on cosmetic products. Official Journal of the European Union No. L342, 22.12.2009.

11. European Commission (EC), 2011. Recommendation of 18 October 2011 on the definition of nanomaterial. 2011/696/EU. Official Journal of the European Union No. L275, 20.10.2011.

12. European Commission (EC), 2017. Regulation (EU) 2017/745 of the European Parliament and of the Council of 5 April 2017 on medical devices, amending Directive 2001/83/EC, Regulation (EC) No 178/2002 and Regulation (EC) No 1223/2009 and repealing Council Directives 90/385/EEC and 93/42/EEC. Official Journal EU No. L117/1 5.5.2017.

13. European Commission (EC), 2018. Commission regulation (EU) 2018/1881 amending Regulation (EC) No 1907/2006 of the European Parliament and of the Council on the Registration, Evaluation, Authorisation and Restriction of Chemicals (REACH) as regards Annexes I, III,VI, VII, VIII, IX, X, XI, and XII to address nanoforms of substances. Official Journal of the European Union No. L308/1, 4.12.2018. 
14. European Medical Agency (EMA), 2006. Reflection Paper on Nanotechnology-Based Medicinal Products for Human Use. https://www.ema.europa.eu/en/documents/regulatoryprocedural-guideline/reflection-paper-nanotechnology-based-medicinal-products-humanuse en.pdf/ (accessed 10.02.2020.).

15. European Medical Agency (EMA), 2012. Reflection Paper on the Pharmaceutical Development of Intravenous Medicinal Products Containing Active Substances Solubilised in Micellar Systems. https://www.ema.europa.eu/en/documents/scientificguideline/reflection-paper-pharmaceutical-development-intravenous-medicinal-productscontaining-active_en.pdf/ (accessed 10.02.2020.).

16. European Medical Agency (EMA), 2013a. Reflection Paper on Surface Coating: General Issues for Consideration Regarding Parental Administration of Coated Nanomedicine Products. https://www.ema.europa.eu/en/surface-coatings-general-issues-considerationregarding-parenteral-administration-coated/ (accessed 10.02.2020.).

17. European Medical Agency (EMA), 2013b. Reflection Paper on the Data Requirements for Intravenous Liposomal Products Developed with Reference to an Innovator Liposomal Product. https://www.ema.europa.eu/en/data-requirements-intravenous-liposomal-productsdeveloped-reference-innovator-liposomal-product/ (accessed 10.02.2020.).

18. European Medical Agency (EMA), 2014. Joint MHWL/EMA Reflection Paper on the Development of Block Copolymer Micelle Medicinal Products. https://www.ema.europa.eu/en/development-block-copolymer-micelle-medicinal-products/ (accessed 10.02.2020.).

19. European Medical Agency (EMA), 2015. Reflection Paper on the Data Requirements for Intravenous Iron-Based Nano-Colloidal Products Developed with Reference to an Innovator Medicinal Product. https://www.ema.europa.eu/en/data-requirements-intravenous-ironbased-nano-colloidal-products-developed-reference-innovator/ (accessed 10.02.2020.). 
20. European Union (EU), 2011a. Regulation (EU) No 1169/2011 of the European Parliament and of the Council of 25 October 2011 on the provision of food information to consumers. Official Journal of the European Union No. L304, 22.11.2011.

21. European Union (EU), 2011b. Commission Regulation (EU) No 10/2011 of 14 January 2011 on plastic materials and articles intended to come into contact with food. Official Journal of the European Union No. L12, 15.1.2011.

22. European Union (EU), 2012. Regulation (EU) No 528/2012 of the European Parliament and of the Council of 22 May 2012 concerning the making available on the market and use of biocidal products. Official Journal of the European Union L 167, 27.6.2012.

23. European Union (EU), 2015. Regulation (EU) 2015/2283 of the European Parliament and of the Council of 25 November 2015 on novel foods, amending Regulation (EU) No 1169/2011. Official Journal of the European Union No. L327, 11.12.2015.

24. Gottardo S., Alessandrelli M., Amenta V., Rambabu A., Barberio, G. Bekker C., et al., 2017. NANoREG framework for the safety assessment of nanomaterials, JRC Science for Policy Report, EUR 28550 EN, doi 10.2760/245972

25. Hafner, A., Lovrić, J., Lakoš, G.P., Pepić, I., 2014. Nanotherapeutics in the EU: an overview on current state and future directions. International Journal of Nanomedicine 9, 1005-1023.

26. Hernan Perez de la Ossa, D., 2014. Quality Aspects of Nano-Based Medicines. SME Workshop: Focus on Quality for Medicines Containing Chemical Entities. http://www.ema.europa.eu/docs/en_GB/document_library/Presentation/2014/04/WC500165 444.pdf/ (accessed 10.02.2020.).

27. Kaur, I.P., Kakkar, V., Deol, P.K., Yadav, M., Singh, M., Sharma, I., 2014. Issues and concerns in nanotech product development and its commercialization. Journal of Controlled Release 193, 51-62.

28. International Organization for Standardization (ISO), 2017. Biological evaluation of medical devices - Part 22: Guidance on nanomaterials. ISO/TR 10993-22:2017. ICS 11.100.20 
29. Lupu A.R. and Popescu T., 2013. The noncellular reduction of MTT tetrazolium salt by $\mathrm{TiO} 2$ nanoparticles and its implications for cytotoxicity assays. Toxicology in vitro, 27(5), $1445-1450$

30. Musazzi, U.M., Marini, V., Casiraghi, A., Minghetti, P., 2017. Is the European regulatory framework sufficient to assure the safety of citizens using health products containing nanomaterials? Drug Discovery Today 22 (6), 860-872.

31. Nystrom, A.M., Fadeel, B., 2012. Safety assessment of nanomaterials: implications for nanomedicine. Journal of controlled release 161, 403-408.

32. Organization for Economic Co-operation and Development (OECD), 2013.

Recommendation of the Council on the Safety Testing and Assessment of Manufactured Nanomaterials. Paris: Recommendation C(2013)107, amended 30/05/2017. https://legalinstruments.oecd.org/en/instruments/OECD-LEGAL-0400 (accessed 10.02.2020.).

33. Organization for Economic Co-operation and Development (OECD), Series on the Safety of Manufactured Nanomaterials No. 63, 2016. Physical-Chemical Parameters: Measurements and Methods Relevant for the Regulation of Nanomaterials. Paris: ENV/JM/MONO (2016)2. http://www.oecd.org/officialdocuments/publicdisplaydocumentpdf/?cote=env/jm/mono(201 6)2\&doclanguage $=$ en (accessed 10.02.2020.).

34. Pita, R., Ehmann, F., Papaluca, M., 2016. Nanomedicines in the EU-Regulatory Overview. AAPS Journal 18 (6), 1576-1582.

35. Scientific Committee on Consumer Safety (SCCS), 2019. Guidance on the Safety Assessment of Nanomaterials in Cosmetics. Brussels: SCCS 611/19. 30-31 October 2019. https://ec.europa.eu/health/sites/health/files/scientific committees/consumer safety/docs/scc s_o_233.pdf (accessed 10.02.2020.). 
36. Scientific Committee on Emerging and Newly Identified Health Risks (SCENHIR), 2006. The Appropriateness of Existing methodologies to Assess the Potential Risks Associated with Engineered and Adventitious Products of Nanotechnologies. Modified Opinion (After Public Consultation) 002/05.

37. Scientific Committee on Emerging and Newly Identified Health Risks (SCHENIR), 2009. Risk Assessment of Products of Nanotechnologies. Brussels: Opinion adopted on 19 January 2009.

38. Scientific Committee on Emerging and Newly Identified Health Risks (SCENIHR), 2015. Guidance on the Determination of Potential Health Effects of Nanomaterials Used in Medical Devices. Opinion adopted on 6 January 2015.

39. Soares, S., Sousa, J., Pais, A., Vitorino, C., 2018. Nanomedicine: Principles, Properties, and Regulatory Issues. Frontiers in Chemistry 6, 360.

40. Tinkle, S., McNeil, S.E., Mühlebach, S., Bawa, R., Borchard, G., Barenholz, Y.C., et al., 2014. Nanomedicines: addressing the scientific and regulatory gap. Annals NewYork Academy of Science 1313, 35-56.

41. Van Teunenbroek, T., Baker, J., Dijkzeul, A., 2017. Towards a more effective and efficient governance and regulation of nanomaterials. Particle and Fibre Toxicology 14, 54 


\section{Conclusions}

Although the progress in the application of nanomaterials in theranostics and tissue engineering has been dramatic and successful, several main challenges remain prior to their widespread adoption in the clinical arena. First, a deeper understanding of the mechanisms and pathways underlying the intracellular uptake and fate of the nanosystems is necessary. Indeed, current systems could face limitations including quick clearance by the immune system and low selectivity and cell targeting, together with difficulties in crossing biological barriers. At the same time, safety concerns should also be taken into high consideration. Nanotoxicity has emerged in parallel with nanomedicine with the purpose of studying the potential negative impact of nano-objects on biological systems. Nanotoxicity should be addressed not only targeting patient safety, but also from a wider perspective embracing the whole up to their disposal. Manufacturing of nanosystems represents another huge challenge. Indeed, large-scale manufacturing is necessary for the reproducible and consistent implementation of nanotechnologies in medicine. It is well-known, however, that synthetic yield is far higher at the laboratory scale than at an industrial one, where bulk properties disfavor the formation of new surfaces. Consequently, scaling up laboratory or pilot technologies still needs to improved, to reach precise control over chemical composition and aggregation, as well as the sustainability of the entire chemical process. These advances should positively impact on economic and financial barriers which currently pose an impediment to the widespread clinical application of nanosystems. We expect the next decade to see a further translation of the scientific knowledge of nanotechnologies to the healthcare industry, resulting in improved diagnosis and early treatment of diseases. 
